
929.2

$\mathrm{J} 774 \mathrm{~h}$

2016650

REYNOLOS HISTORICAT

GENEALOGY COLLECTION 


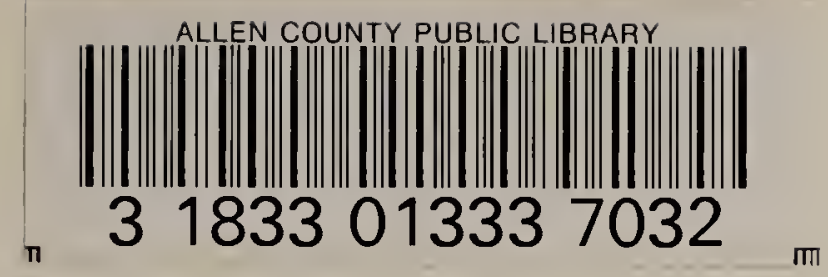







\title{
THE JOSEPH GENEALOGY
}

\author{
compiled by
}

(Mr.) Vernon Hitchens

1963

\author{
(1) 048 \\ $\begin{array}{lllll}78 & 8318 & 9\end{array}$
}




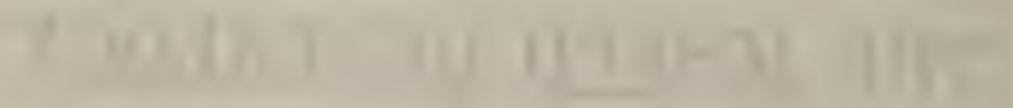

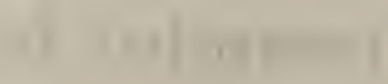

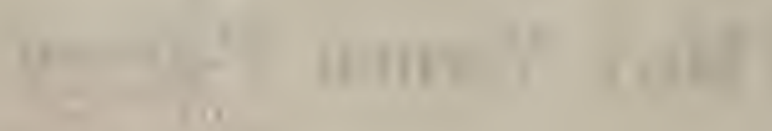

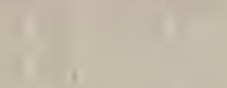

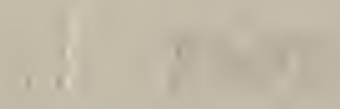

$$
\begin{aligned}
& +4 \\
& \text { ! } \\
& \text { 7. } 15 \times 4+5
\end{aligned}
$$




\section{0}

I would like to take this opportunity

to thank all who helped make the book a success. There are still many Joseph families we have no information about. Information has come from all parts of Del.

\section{Vernon Ilitchens}


The Joseph family has resided in Delaware for many years. Before Revolutionary War gave birth to a new nation Joseph Joseph was the first Joseph who settled in Sussex County, Delaware. He was a native of Scotland. One of his children was Jeremiah, who was the father of ten children. Elisha Joseph, Esther Joseph, Heze. kiah, Joseph, Jr., Jonathan, Lavina, 'William,' Zackariah,' Nathan H., "Elizabeth.

Information received from the Walls Book. We will begin with the third child of Jeremiah. Hezekiah Joseph married Nancy Walls, Born 8-81769.

William E. Joseph, Born 6-1-1811, Died 2-251855 married Elizabeth Walls, Born 10-12-1812, died 6-26-1892. She is buried in St. Georges Chapel Cemetery. Daughter of Nehemiah and Elizabeth E. (Clark) Walls.

Eliza Ann Joseph Born 5-19-1835, died 6-28. Married 4-5-1859 Gilly S. Walls 1-30-1830, 5-141903. Daughter of John and Harriett "Walls) Walls). Both buried in St. Johns Methodist Cemetery near Georgetown.

Hannah E. Walls, Born 3-3-1861, died 5-291948. Married Josiah James Simpler, Born 7-181859, 7-6-1923. Son of Josiah and Sarah H. (Atkins) Simples. Both buried in St. Johns Methodist Cemetery.

Mary E. Simpler, Born 1887. Married 12-241907. Golda Dodd, 1883 - 1949. Buried St. Johns. Two children, Miles E. Dodd, Born Trifl? Married 2-14-1953, Elizabeth Megee, buin 2-41914. Daughter of Herbert and Lola (Legates) Megee. No issue. Leona Mae Dodd, 1916-1917. Buried at St. Johns Cemetery.

Lida Mae Simpler, married Harvey J. Gordy, born 10-4-1887, died 3-24-1925. Buried Union Cemetery, Georgetown. Son of Benton Bell and 


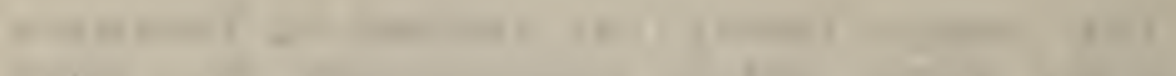

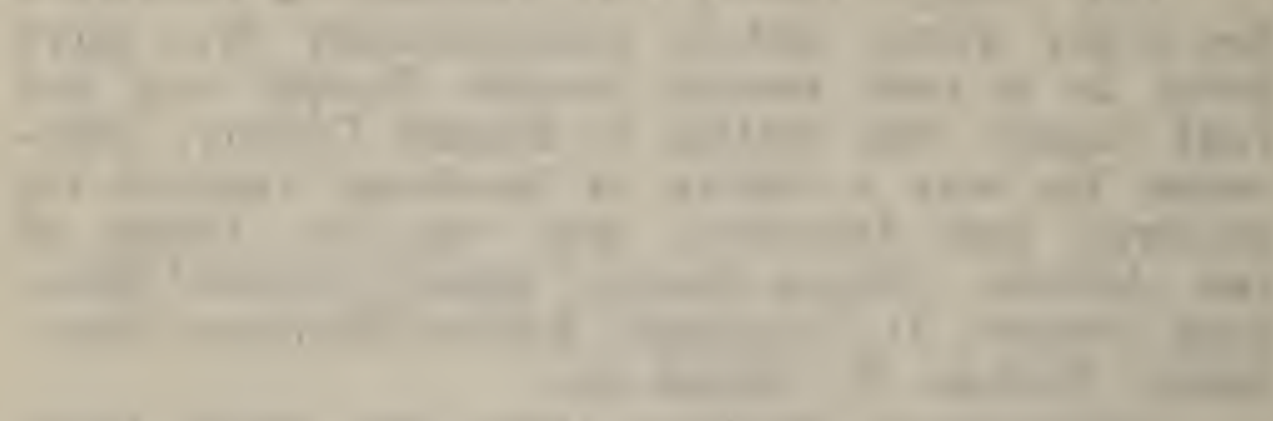
10 1 10 10 $1+200$ $1+20$ (1) 100 10

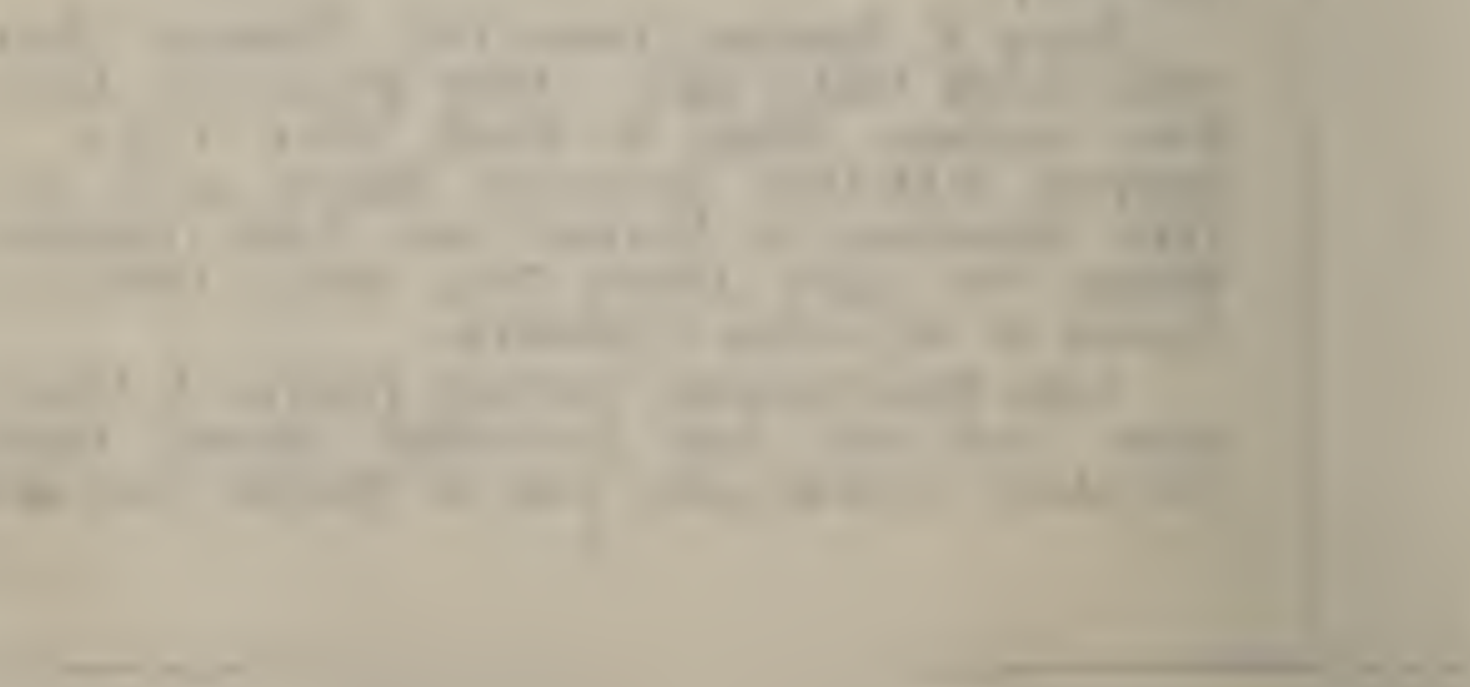


Annie E. (Timmons) Gordy. Harold B. Gordy, Born 6-30-1920, died 2-22-1951. Married 1944 Elizabeth Robbins Stroeckecker, Born 5-5-1921. Two children, Harold B. Gordy, Jr., Born 4-231915. Elizabeth S. Gordy, Born 1-16-1922.

Esther M. Gordy, Born 1-16-1922. Married 193. Orlando C. King 5-5-1913. Son of John R. and Margaret M. (Calhoun) King. Three children, Janet Lee King, born 11-4-1941: Irvin B. King, born 12-31-1943; Orlando C. King, born 11-6-1947.

This information received from Mr. Edgar Simpler of Pennsylvania.

Edgar L. Simpler, born 2-1-1895. Married 25-1916, Leona Johnson, born 2-11-1894. Daughter of George W. and Arabella (Fosgue) Johnson. Emma E. Simpler, born 1-2-1921. Married 2-5-19:38 Howard Lee Haddock, Jr., born 6-28-1919. Son of Howard Lee and Winifred Adaline (Adkins) Haddock. Two children, Brenton H. Haddock, born 1-6-1939. Married 5-5-1962 Priscilla Hoopes, born 11-27-1937. Mary Louise Haddock, born 1-4-1942.

Edgar L. Simpler, Jr., born 6-15-1923. Married 5-26-1951 Zeneda A. Back, born 10-10-1928. Daughter of Berlyn Clay and Lila D. (Dunk) Black. Four children, Carol Marie, born 7-7-1955; Wade Black, born 4-10-1958; Ann Elizabeth, born 5-311959; Linda May, born 10-1-1960.

Leona Johnson Simpler, born 6-16-1933. Married 9-8-1956, Lawrence Geo. Edw. Sucker son of Virgil Victor and Ida F. (Smith) Sucker. No issue.

Ulysses L. Walls, 1864-1943. Married 1st. Isa Lank, born 2-21-1875, died 6-29-1911, son of Samuel J. and Sarah H. (Simpler) Lank. One child, Samuel Walls, 1898-1899. He married 2d., Mary Hellene Johnson, born 1886, died 1933. All buried in St. Johns Cemetery. Son of 'Thomas and Hetty (Perry) Johnson.

Juseph E. Walls, born 12-29-1871, died 8-6- 


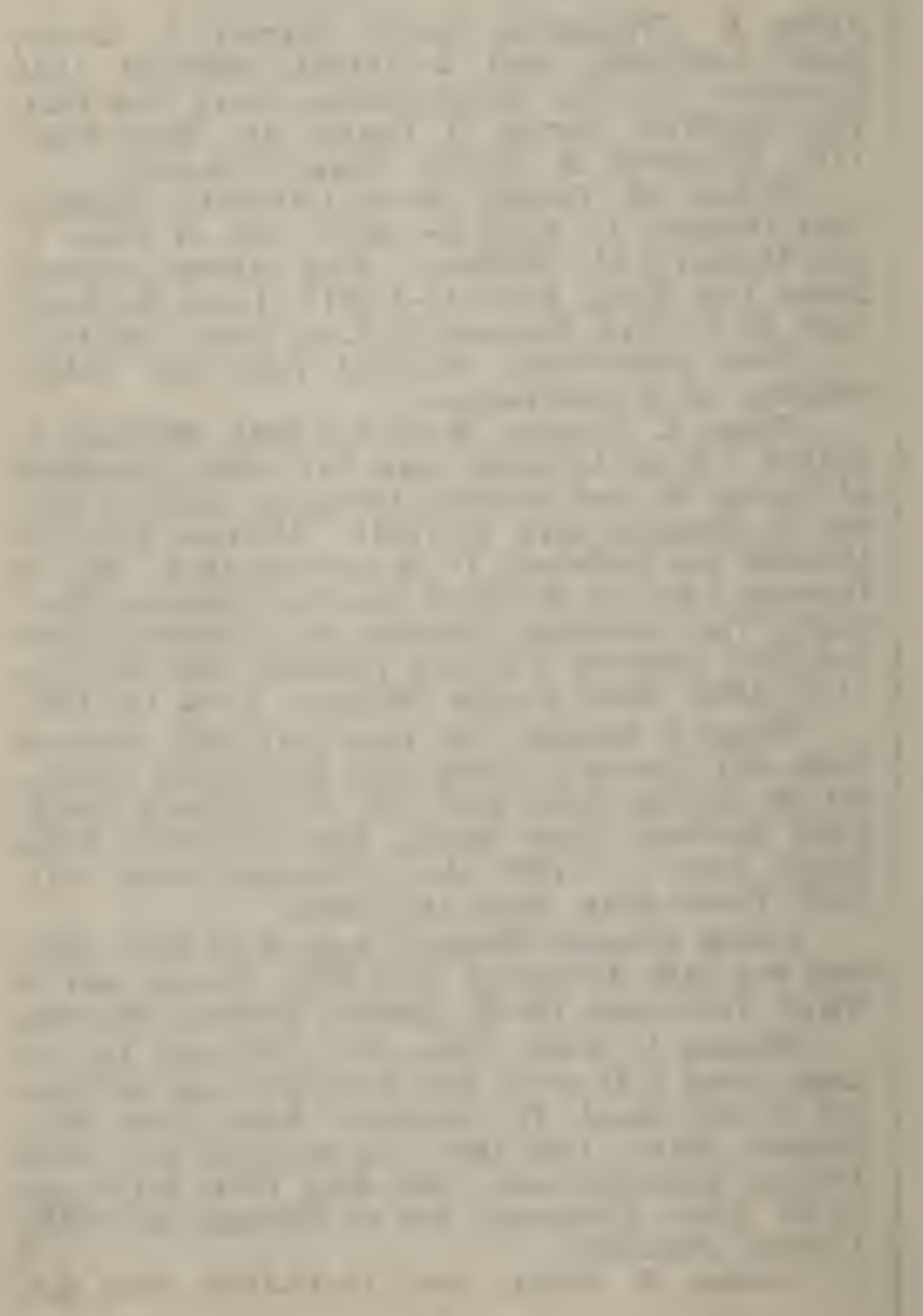


1940. Married 1893 Glennie E. Truitt, born 9-281874, died 2-28-1938, son of John and Oliva (Wright) Truitt. Both are buried Odd Fellows Cemetery, Milford, Delaware. One child, Ethel Walls, born 9-23-1900. Married William H. Ackerman, born 9-7-1899. No issue. Son of Oscar and Minnie W. (Davis) Ackerman.

Emily Y. Joseph, born 4-21-1837, died 8-:201916. Buried St. Johns Church Cemetery. Married David M. Prettyman, son of James and Lydia (Holland) Prettyman. She married 2d, Mary E. Prettyman, born 1865, died 1918. Married Trui't Pettyjohn, born 1862: died 1921, son of 'Truitt and Mary E. (Reynolds) Pettyjohn. Golda A. Pettyjohn, born 1-7-1890. Married Harriet E. Rust, born 1893, daughter of Hammond and Mary L. (Parker) Rust. David Ralston Pettyjohn, born 6-9-1041 married Emma Mildred Dorey, borr 4-16-1988, daughter of Wm. B. and Cora (Johnson) Dorey. Two girls. 1. Myrna Gail Pettyjohn, born 2-1941, married 11-5-1960 Floyd Megee, Jr. 2-3-1939. Three children, Lisa Gaye, Ron and Wm., twins, Born 2. Norma Gaye Pettyjohn. Golda A. Pettyjohn, Jr. born 4-12-1925. Married June White, born 11-6-1928, daughter of George White and Nellie E. Isaacs. Two sons, G. Jeff, born 9-21-1954 and Dean R., born 5-13-1958. The above information received from Mrs. Mildred Pettyjohn.

Virginia Emily Pettyjohn, born 1892, died 1894, buried at St. Johns Cemetery. Anna Emily Pettyjohn, born 7-19-1895. Married William HI. Truitt, son of Henry and Alice (Hudson) Truitt. One son, David Truitt, married, one child.

Horace R. Pettyjohn, born 10-25-18?8, dicd 4-13-1949. Married 7-30-1926. Helen Lank, born 11-25-1908, daughter of Edwin and Georgianna (Walls) Lank. 


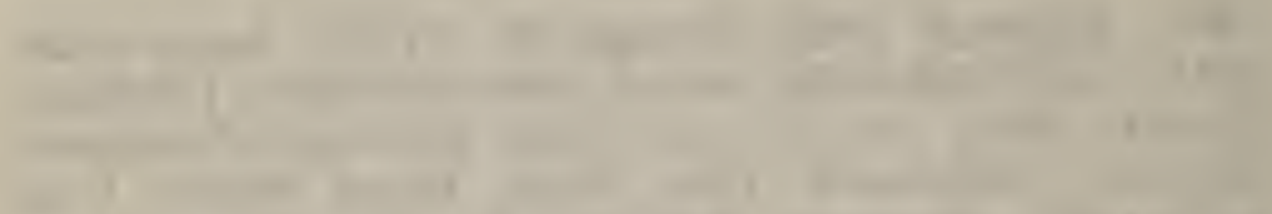

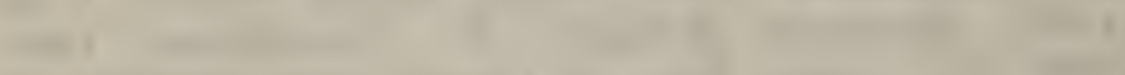

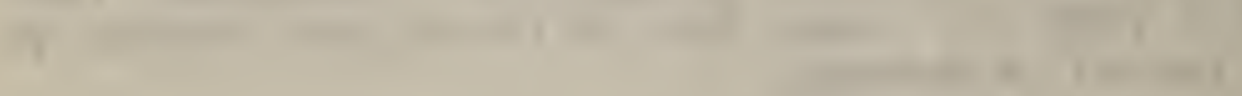

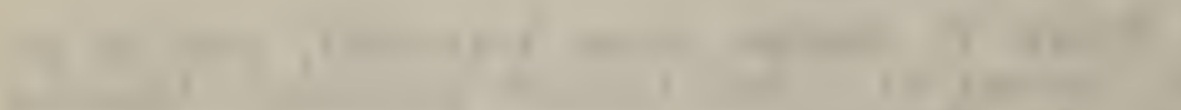

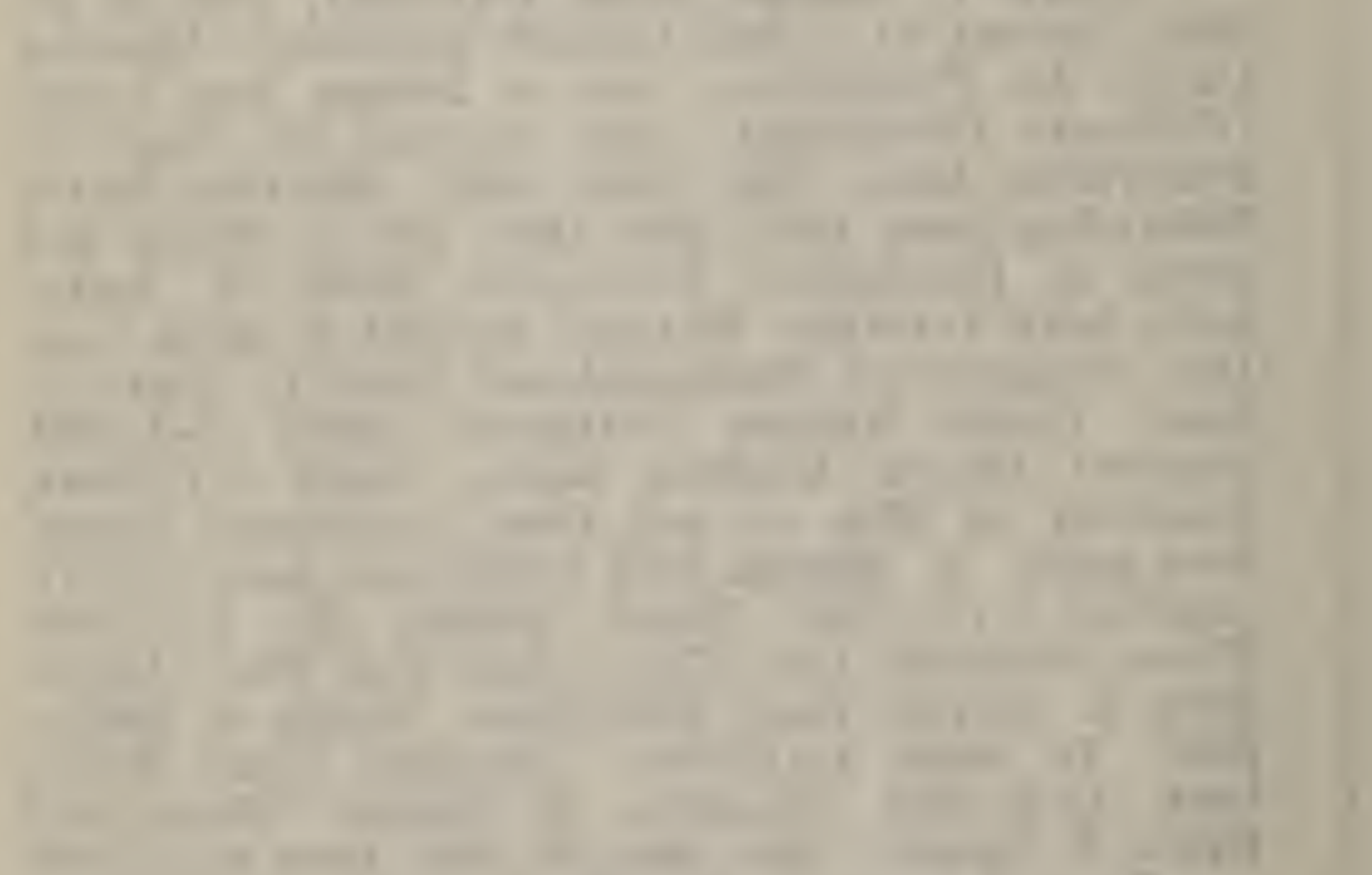

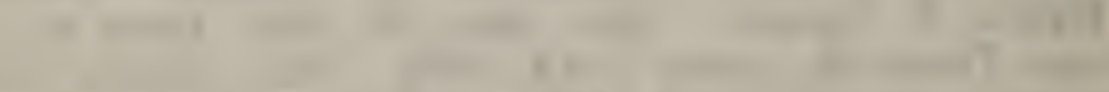
(2)

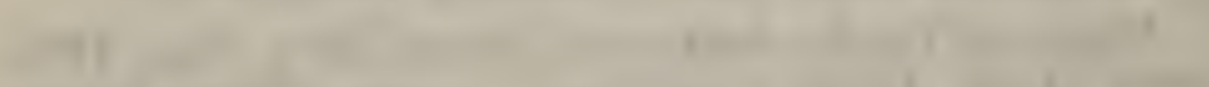

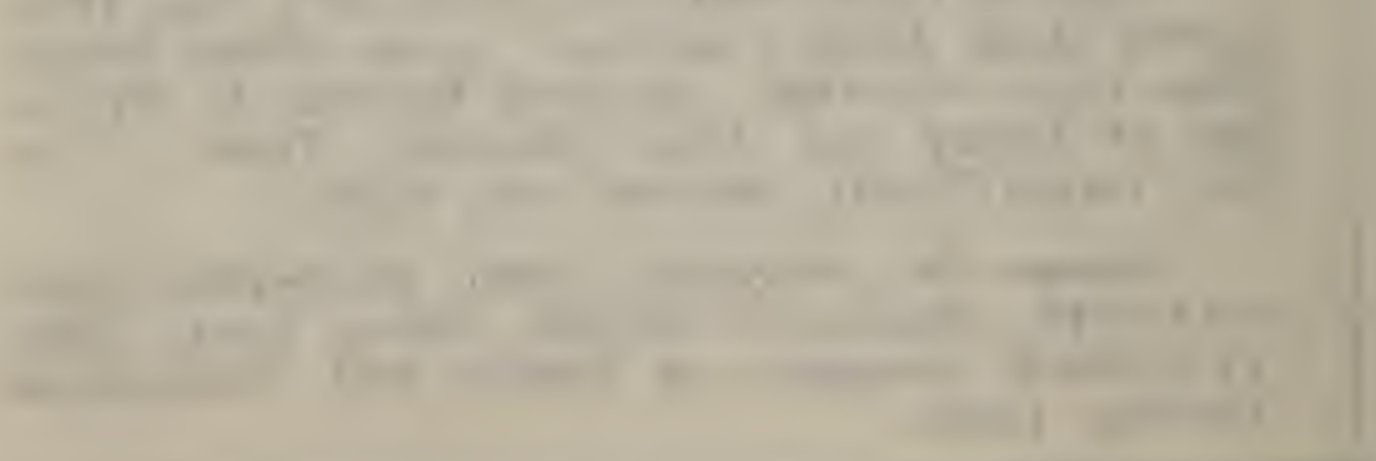


Harold Truitt Pettyjohn, born 1-7-1927. Married 3-19-1946, Agnes Lorraine Prudell, born 7-21926, daughter of Anthony and Mabel (Knudson) Prudell. Three chiluren, Ronald Truitt, 1-12-1947; Rene Lynn, 7-4-1950; Barry Wayne, 7-2-1954.

Mary Ann Pettyjohn, born 3-25-1928, married 4-9-1949, Wm. Joseph Maloney, born 1-12-1925, son of James and Emily (Smith) Maloney. 'Two children, John Wm., born 4-22-1952; Steven Q., born 2-1-1950. This information received from the Walls book.

Norman R. Pettyjohn, born 6-17-1900, died 310-1901. Buried St. Johns Cemetery.

Robert Prettyman, married 1st. Anna. Married 2d Mary. They resided in Philaclelphia, Pa. and are buried there. Three children by 1 st wife; two children by $2 d$ wife.

Emly Y. Joseph, married $2 d$ George Walls, born 1824, died 1902. Both buried St. Johns Cemetery. No issue by this marriage.

Catherine E. Joseph, born 9-27-1\$40, died 915-1918. Marricd Alfred Marvil, born 1837, died 1924. Both buried St. Johns Cemetery.

Frank W. Marvil, born 1863, died 1932. Married Ella V. Millman, born 1878, One child, Ella Mae Marvil, died in infancy. Buried St. Johns. Alvin and Alfred Marvil.

Annie Marvil, born 1862, died 1946. Married Nath T. Veasey, born 1849, died 1931. One child, Hattie Veasey, married Charles Phillips. Data received from the Walls book.

Thomas A. Joseph, born 12-12-1832, died 529-1905. Married Nancy C., born 10-4-1835, died 1918. Both buried St. Georges Chapel Cemetery Alvin Lee Joseph, born 1859, died 1929. Married. Laura Goslee, born 1861, died 1940. daughter of Fiward and Margaret E. Goslee.

Arzie T. Joseph, married "Nath Washington 


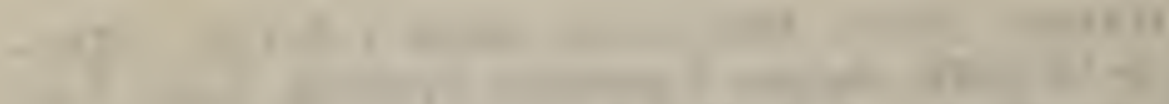

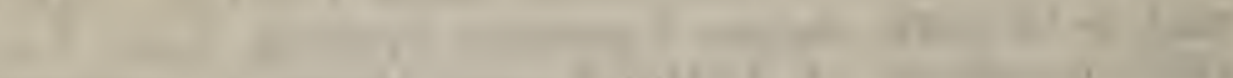

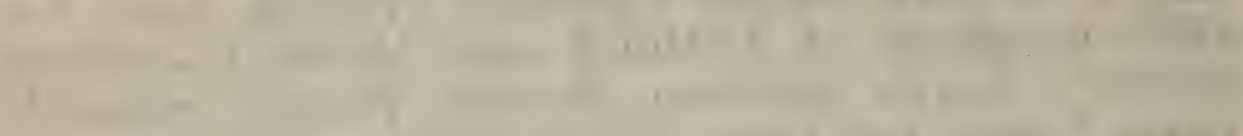

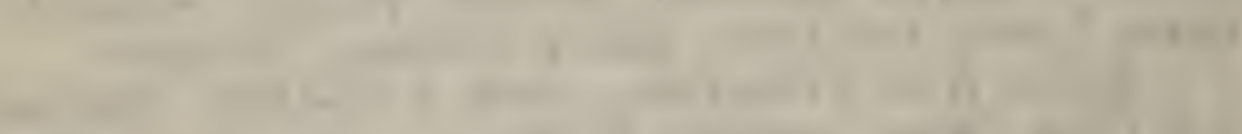

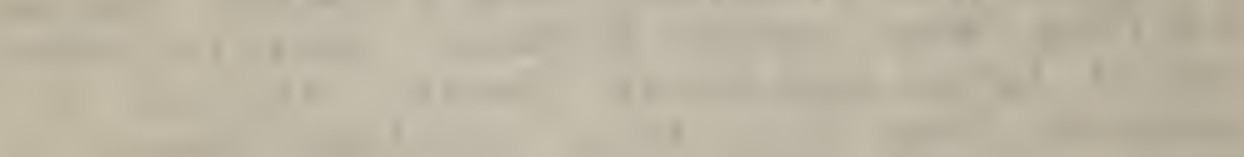

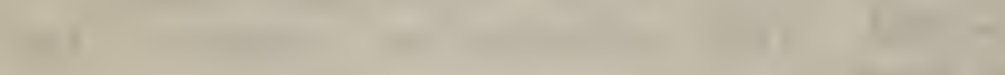

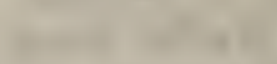

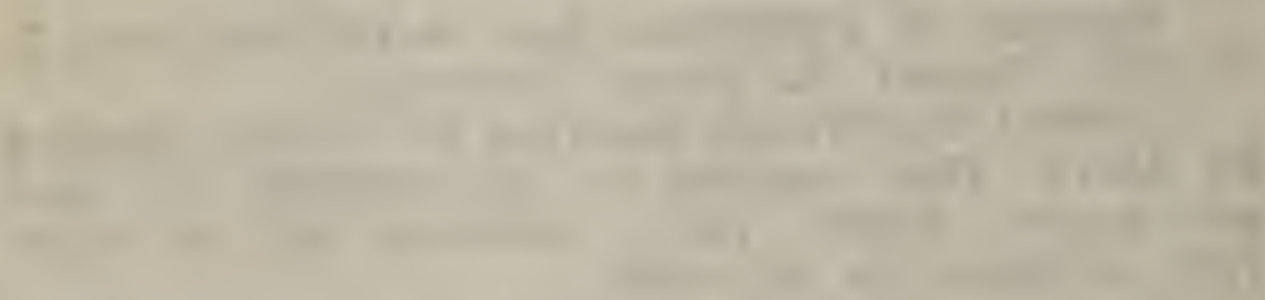

$x^{2}$

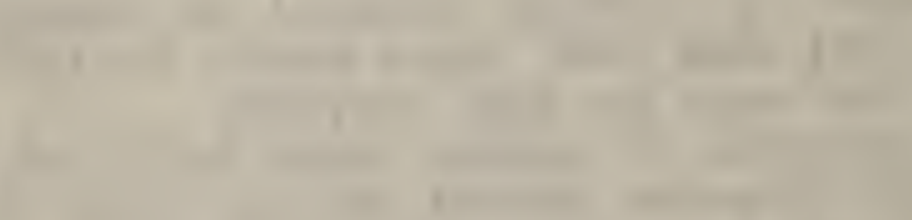

\section{tris}
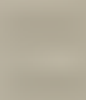

$x^{2}$

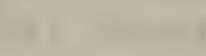

aty

(2n)

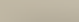

(n)

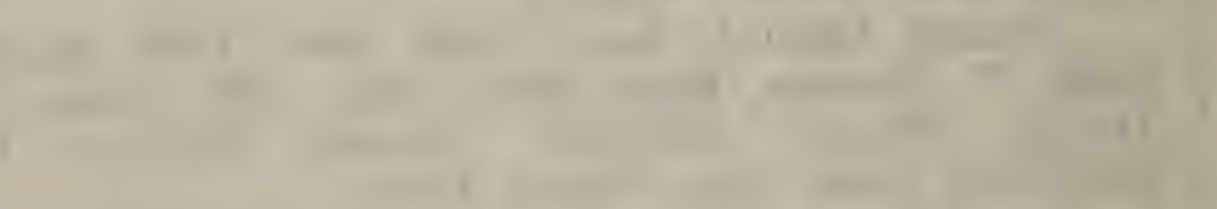

I $=1$

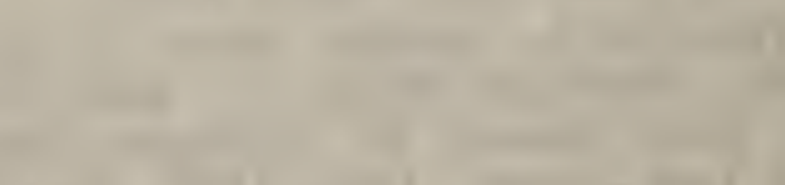

(1)

$-1$

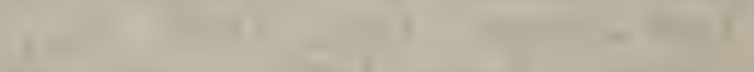

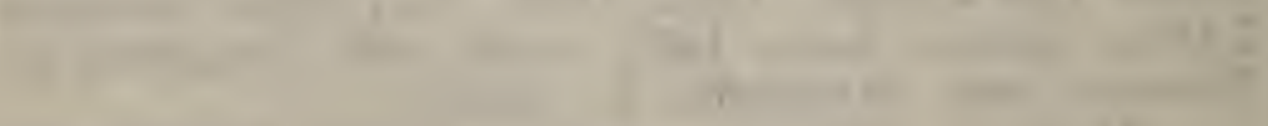

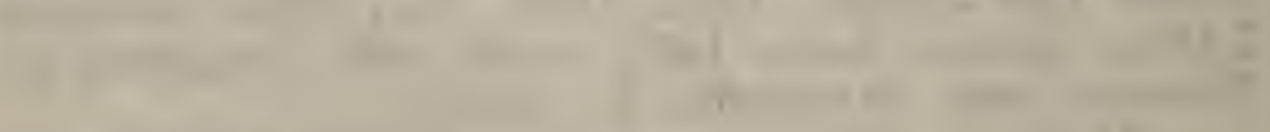

- 
Prettyman, born 8-9-1888, son of NWB and Emma (Burton) Prettyman. Two daughters, Edith Preltyman, born 1917, died 1944. Married Wilbur Lee Rogers, son of George W. and Eva (Hudson) Rogers. One son William Lee Rogers, born 5-11944.

Marion Lee Prettyman, born 12-19-1921. Married Rudolph B. Hastings, son of Harley G. Hastings. Four children Donna, Susan K., Gregory, Holly.

Katherine Joseph, born 1901, died 1958. Married Alphonso Hazzard, born 1875, died 1955. No issue.

Hoyt Joseph, dead when born 1889. All are buried St. Georges Chapel Cemetery.

Laura Joseph, boin 1867, died 1875. Buried St. George Chapel. Harland M. Joseph, born 1868, clied 1948. Married Margaret E. ——, born 1373, died 1957. Margaret E. Joseph, born 1909, dicd 1932, unmarried. All buried at Midway Presbyterian Cenetery near Lewes. G. Leighton Joseph married Helen Taylor. No issue. Ann Joseph married —_ Benedict. I ulu Joseph married Eugene Ackerman. Two daughter, Margaret Ackerman, married Roger Smith. Josephine Ackerman married _ Armstrong. One daughter, Lulu Armstrong. Ben Joseph married Mary Edwards.

Charles I. Joseph, born 2-26-1871, died 9-211946. Married Minnie Ward, born 8-31-1875; died 11-4-1962. Both buried St. George's Chapel Cemetery. Charlotte Joseph married Melvin Brobst. Cne son, David Brobst. Alfred W. Joseph married —. Emma Joseph, born 1873, died 1874. Anna Joseph, born 1875, died 1950. Married Harry $N$. Hart, born 1873, died 1944. Both buried Odd Fellows Cemetery, Milford. Son of Arthur J. and Eliza A. Hart. H. Fleming Hart married Lclia Hart married 1st. Edward Hill. One son. 


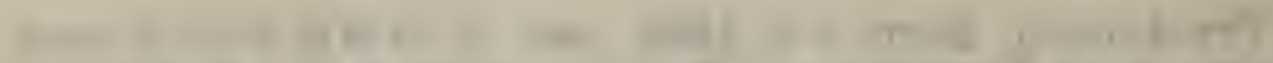

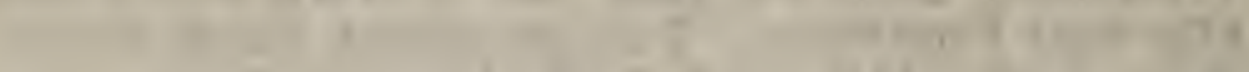

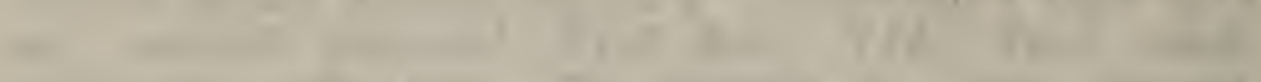

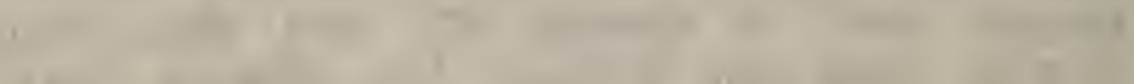

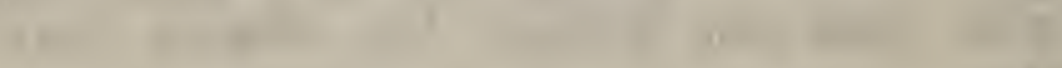

$$
\text { and } 10
$$

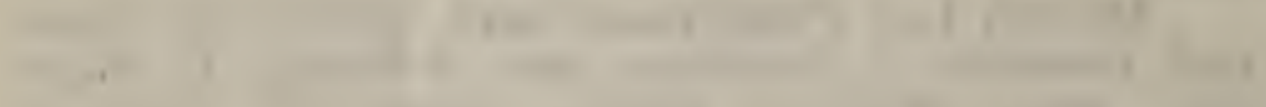

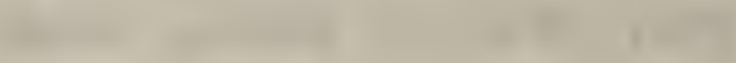

and

1. 14.

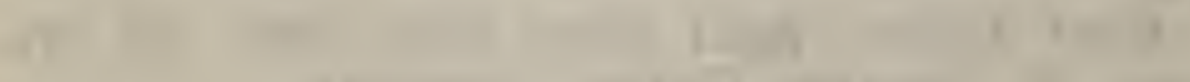
and

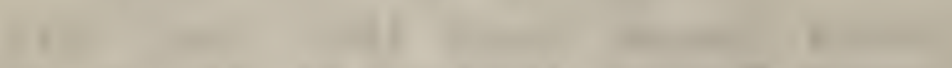

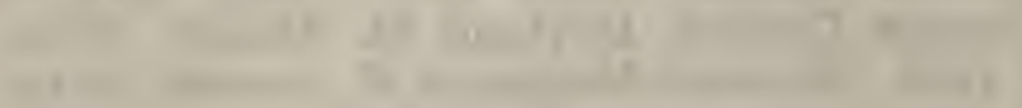

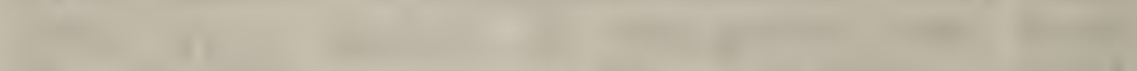

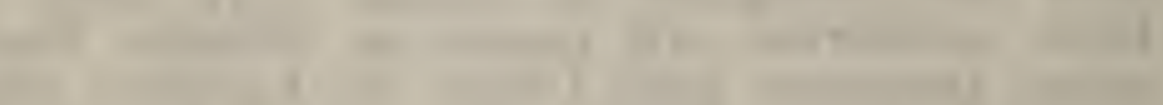

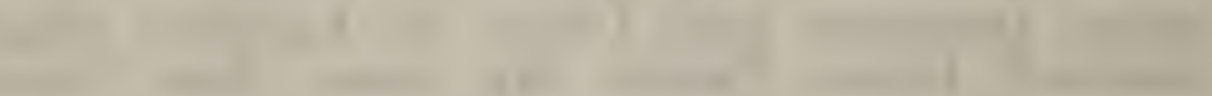
10.

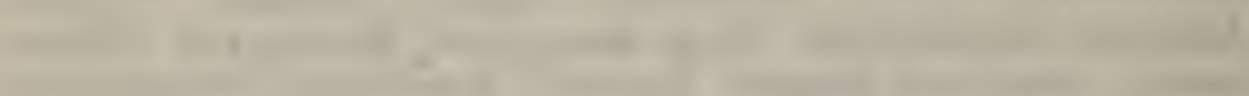
10

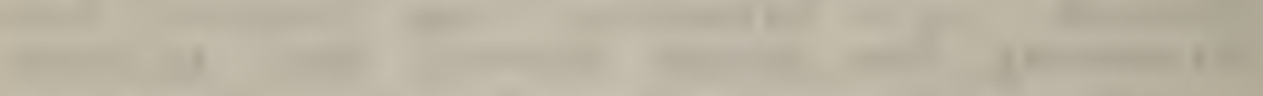

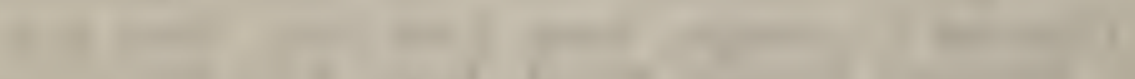

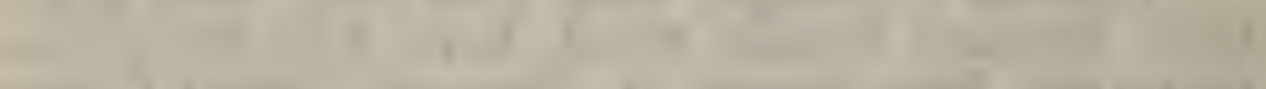

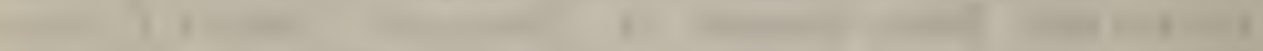

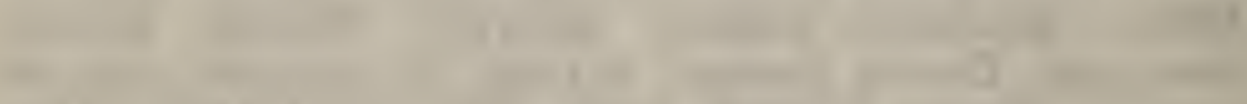
(1)

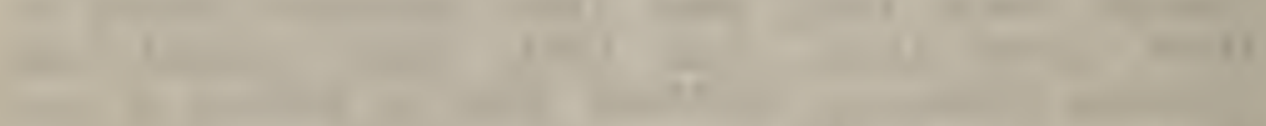

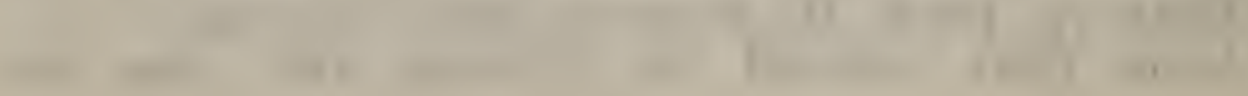


Edward Hill, Jr. She married 2d., Stanley Pontiere. Eugent M. Hart, born 1902, died 1953. Buried Odd Fellows Cemetery, Milford, Delaware. Married Margaret V., born 12-21-1907. One daughter, Mrs. Wm. A. Smith. Edna Hart married Edgar $M$. Welch, born 18ā5, died 1-6-19l0. Buried Odd Fellows Cemetery, Milton, Delaware. Edgar M. Welch, Jr., married Mary Jane Carow daughter of John O. and Frances Carow. Two sons. Mrs. Lisue Carney. Frances Hart married Gordon Saltar.

Amanda Joseph married George Burton. Florence Burton married William Prettyman, son of N. W. B. and Emma (Burton) Prettyman. Laura Joseph married James Marsh. W. Frank Joseph inarried Cora Marvel, born 1869, died 1897.

Ralph Joseph, born 1898, died 1959. Buried St. Georges Chapel Cemetery. Married Maggie Johnson, born 8-8-1897. Lawrence Joseph, born 1920, married Jesse —_. Five children, Lawrence, Jr.; Nancy; Joe Ann; James; Susie Joseph Cora Joseph, born 2-13-1921 married Harry O. Marker, born 7-24-1918. Five children. Harry O., Jr., born 7-28-1943; Maxium, born 6-7-1946; Shirley, born 5-3-1948; Gail, born 4-25-1956; Ronald, born 3-8-1958. Minnie Joseph, born 1923 married Mervin Doughty. Three children. Robert $M$; Karen Sue; Keith L.

Miranda Joseph, born 11-25-1842, died 1218-1906. Married David M. Waples, died 1902, 61 years. Wm. E. Waples, born 1865, died 1931. Married 1st., Clara Fletcher, born 1856, died 4-121889. Buried Midway Presbyterian Cemetery. Daughter of John M. and Susan M. Thompson Fletcher. Wm. E. Waples married 2d., Jennie Morax. Buried Cemetery near Midway. Harry C. Waples married Bertha Layton. No issue. Amanda Waples married John Sockrider, 1885-1951. Buried 


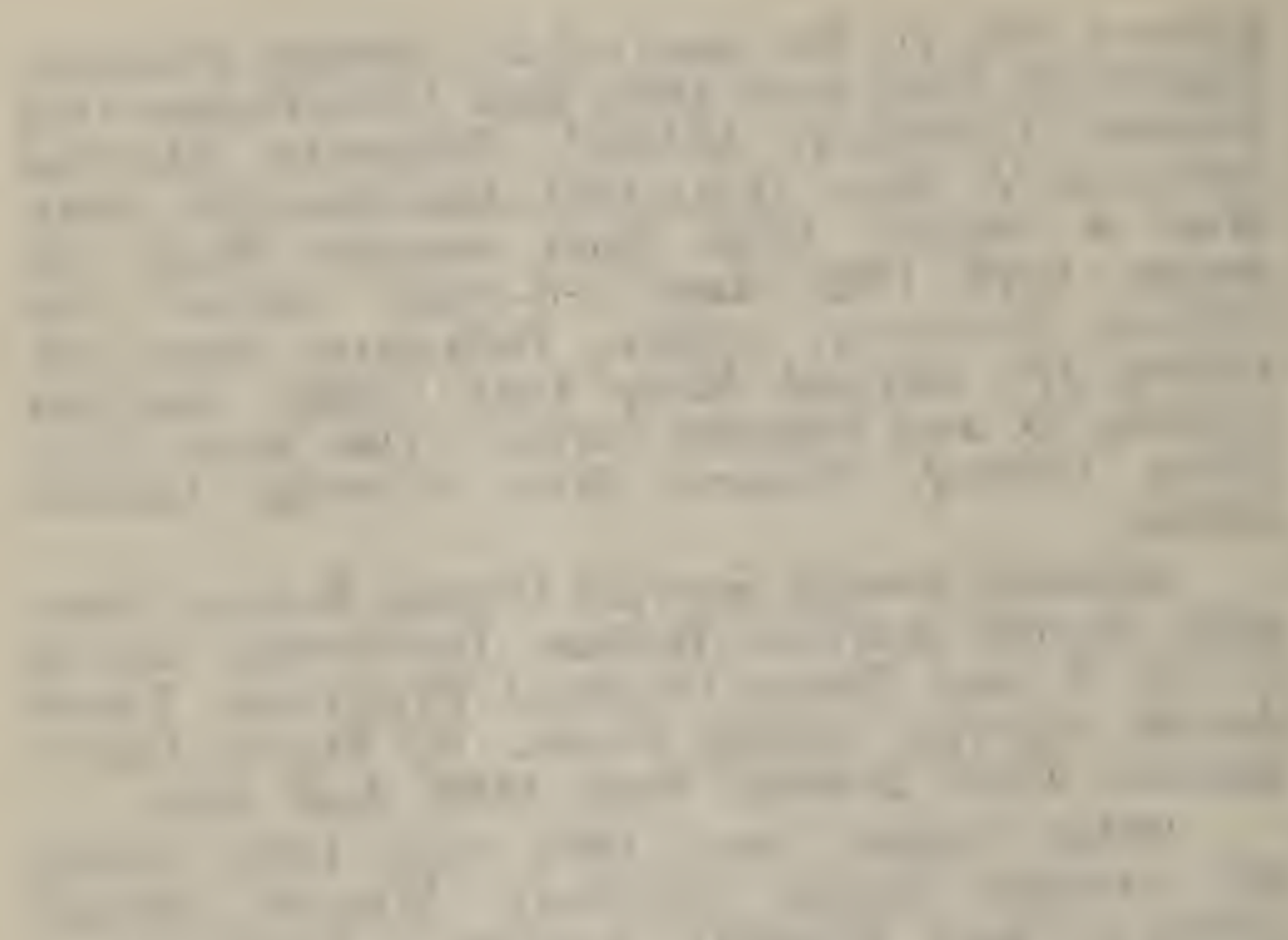

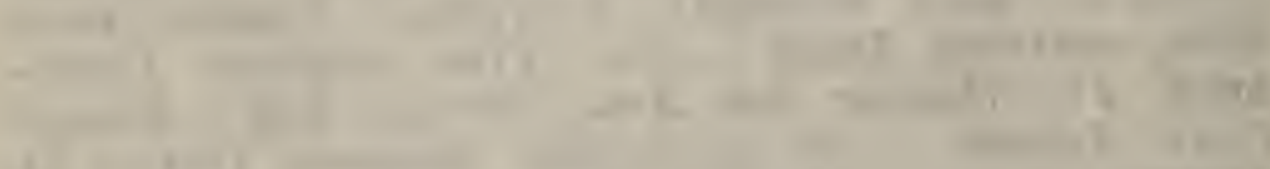

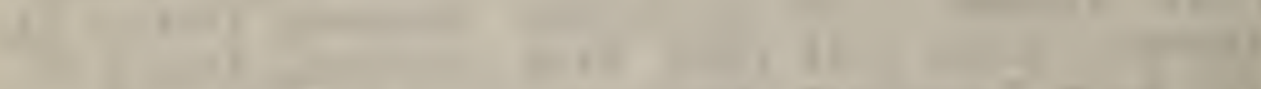

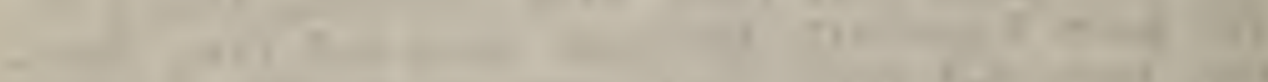

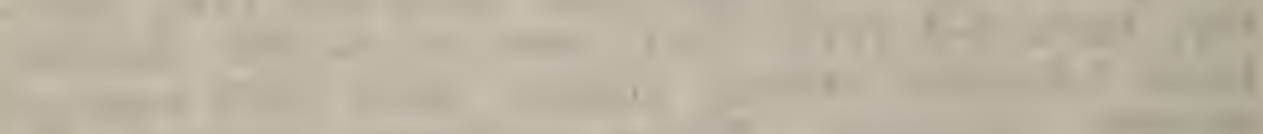

$$
\begin{aligned}
& +1
\end{aligned}
$$

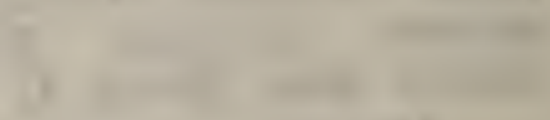

14. I

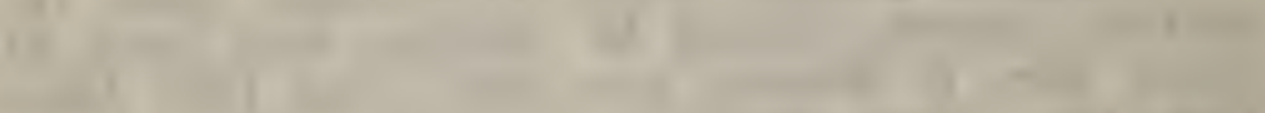

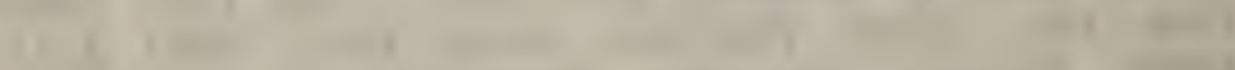

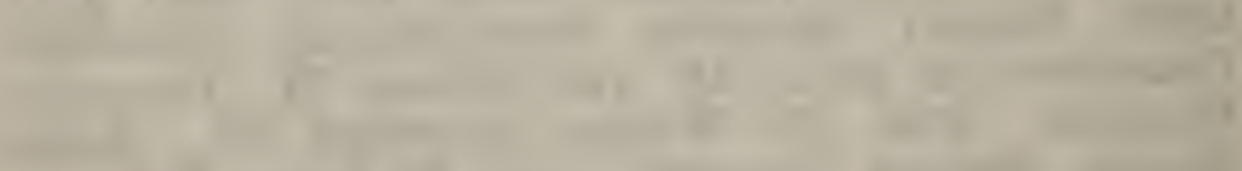

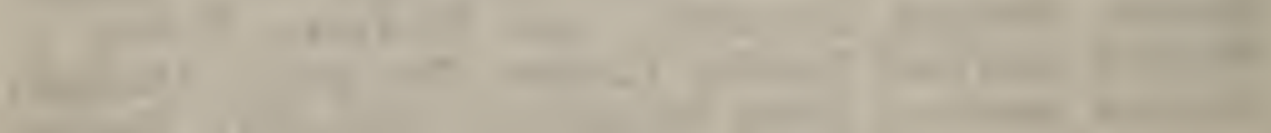


Conleys Chapel Cemetery. Ethel Sockrider married Charles Crockett. One daughter Thelma Crockett married James Schilpt. Two children, Diane and James. Wm. D. Waples, born 11-201894, died 1959. Buried Beaver Dam Cemetery, Harbeson. Married Lida M. Sockrider, born 4-241892, daughter of Samuel and Anna Bell Sockrider. Wm. D. Waples, Jr., born 9-6-1918. Married Mabel Draper. One daughter, Sylvia E. Waples married Ronald Drake. Three children Gary L.; Cystal L.; Barry M. Alice Mae Waples married George E. Hopkins. Four children. Information received from the Walls Book. Richand $R$. Waples married Elsie Houston. Mary Waples married Clarence Carroll. Elva Jane Waples. Georgett Waples.

Wm. E. Waples married 3d. time Emma Hudson, born 1896, died 9123. They had one child, Margaret Waples, died 3-19-1911. He married 4th time Exie Sockrider. No issue. Joseph A. Waples married 1st. Mary Corudule. No issue. Married 2d. Laura Bunheisia. No issue. Clarence D. Waples married 1st. Minnie - Married 2d. Mary Collins. Three children by first wife. Ella -. Waples, born 3-5-1876, died 3-15-1953. Married 1st. James Keane, born 5-29-1900, died 1-8-1923. Both buried Harbeson Cemetery. Married 2d. Abram Sparks. Two children. Harvey C. Waples, died 3-17-1887. Eliza J. Waples, died $9-11-1875,7$ years. Mary E. Waples, died 1865,1 year. All buried at St. George's Chapel Cemetery.

Margaret Joseph, died, 1845. Jane R. Joseph, died 1847. Willard S. Joseph, born 8-20-1949. Willard W. Joseph, born 8-16-1851, died 4-181917 married Evelyn Rust, born 8-10-1859, died 1-18-1929. Both are buried at St. Johns Cemetery Bertha M. Joseph, born 4-23-1879, died 8-13-1957. Married George Walter.Burton, born 1873, died 


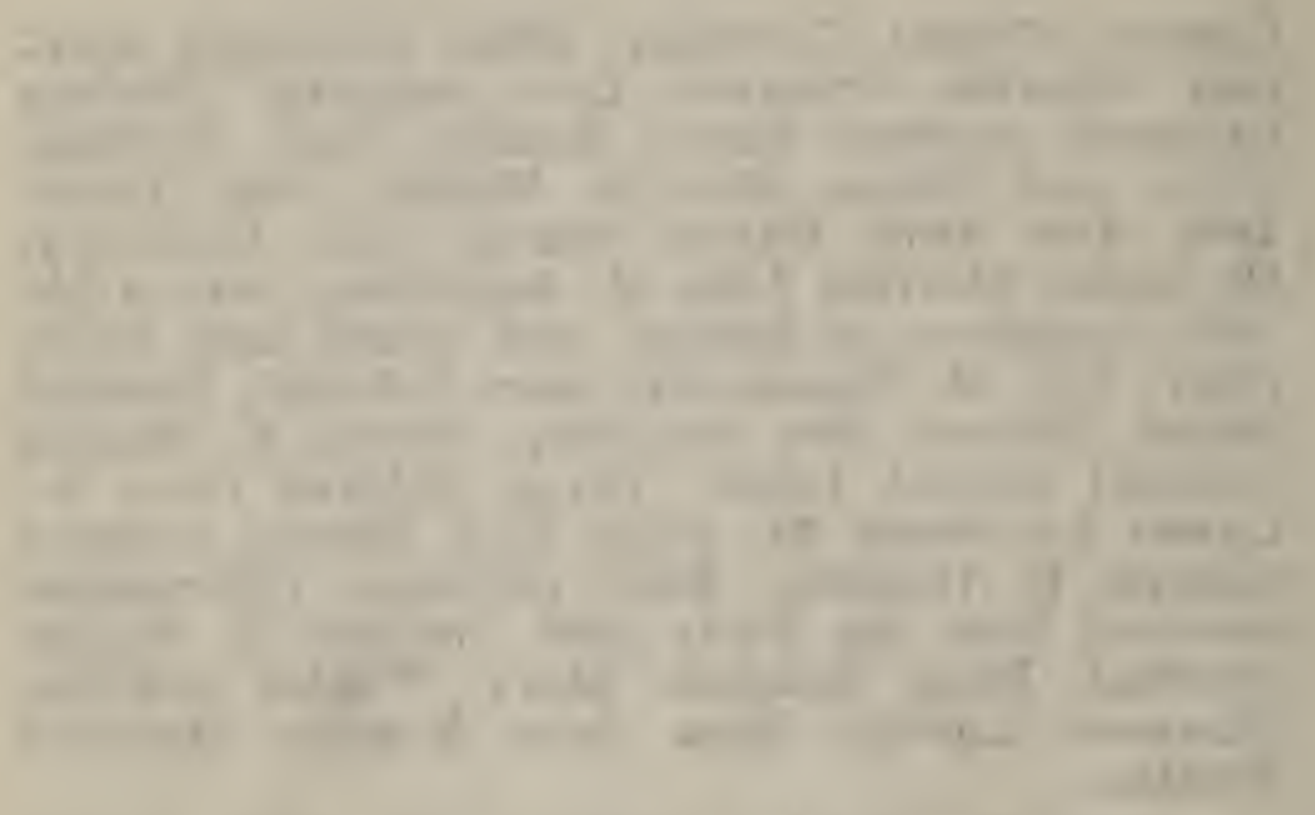

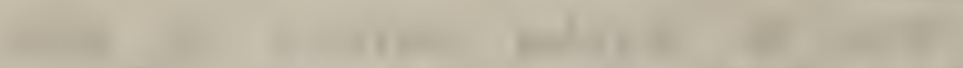

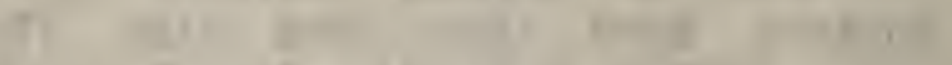

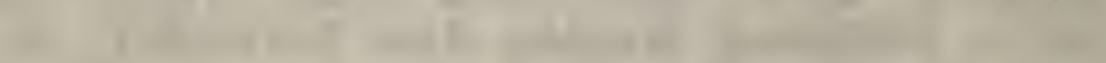

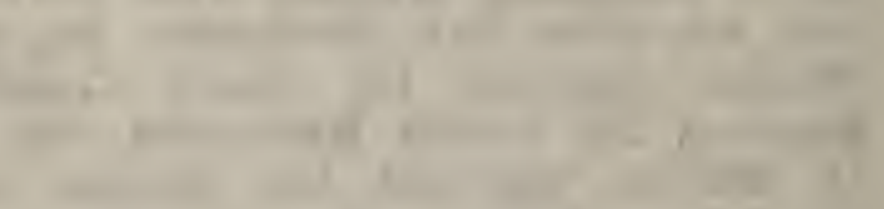

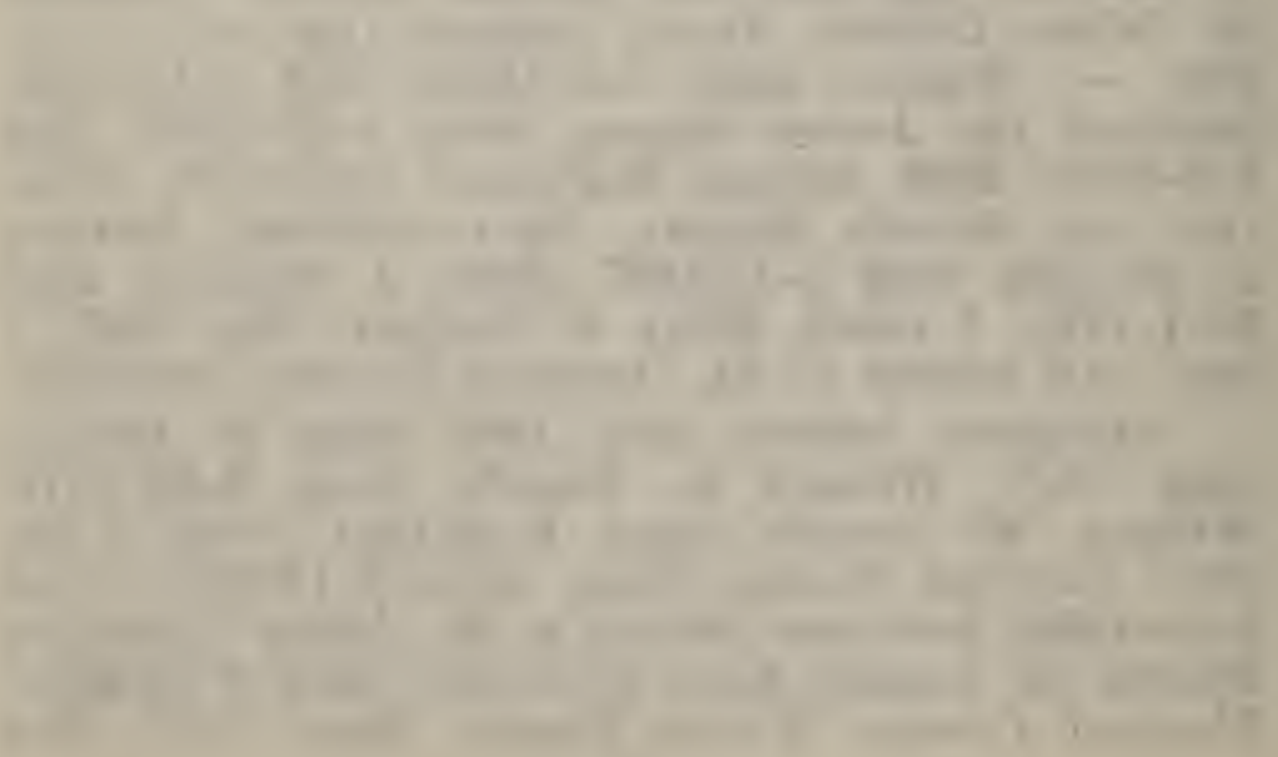


1949. Both are buried in Conley's Chapel Cemetery. Son of Stockley and Hester (Marvel) Burton. Cura M. Burton, born 1900, died 1950. Buried in Cunley's Chapel Cemetery. Married 1st. William Starret. Married 2d. Clarence Atkinson, born 1903. Son of Ben and Lucy Prescott Atkinson. Nellie C. Burton, born 5-3-1905. Married Harry P. Hazzard, born 1899 died 1962. Buried at Conleys. Irene E. Hazzard, born 10-11-1922. Married William Gooner. Fuur children. Herbert Megee Hazzard, born 12-12-1933. Married Marlyn Willard. Four children. Eugene R. IIazzard, born 11-1-1933. Married Joy Dubb. Doris Lee Hazzard married 1st. Hyland Marvel. Two children. Married $2 d$. David Mantyla. Dorothy W. Burton married Clifford U. Messick, born 1910, died 1950. Buried Conley's Chapel Cemetery. Six children.

Daniel $T$. Joseph, born 9-10-1880, died 1-111933. Buried Conley's Cha'pel Cemetery. Married Elizabeth M. Narsh, born 6-21-1891. Daughter of Nath L. and Narvello (Johnson) Marsh. Howard M. Joseph, born 1909. Married Helen M. Moore. No issue. Daughter of Joseph R. and Mary J. Moore. Carlton W. Joseph, born 4-2-1916. Married Juantia Pose, daughter of John H. Pose. C. Pichard Bebe Joseph, born 10-12-1919. Married Marie Green, born 1923. Daughter of Clifford. O. Green and Marie Veasey. Three children, Clifford $O$. Joseph, born 1947. Sandra Joseph, born 1955, died 3-19-1958. Diane Joseph, born 1956 .

Wilmer C. Joseph, born 4-3-1882, died 6-41963. Buried Lewes Methodist Cemetery. Married 14t. Margaret Marsh. No issue. Daughter of Wm. and Thadia (Johnson) Marsh. Married 2d. Minnie Brereton. No issue.

Fred W. Joseph, born 1884, died 1-6-1963. Buried Conley's Chapel Cemetery'. Married Emma Jane Marsh, born 1-29-1887. Daughter of Nath. 


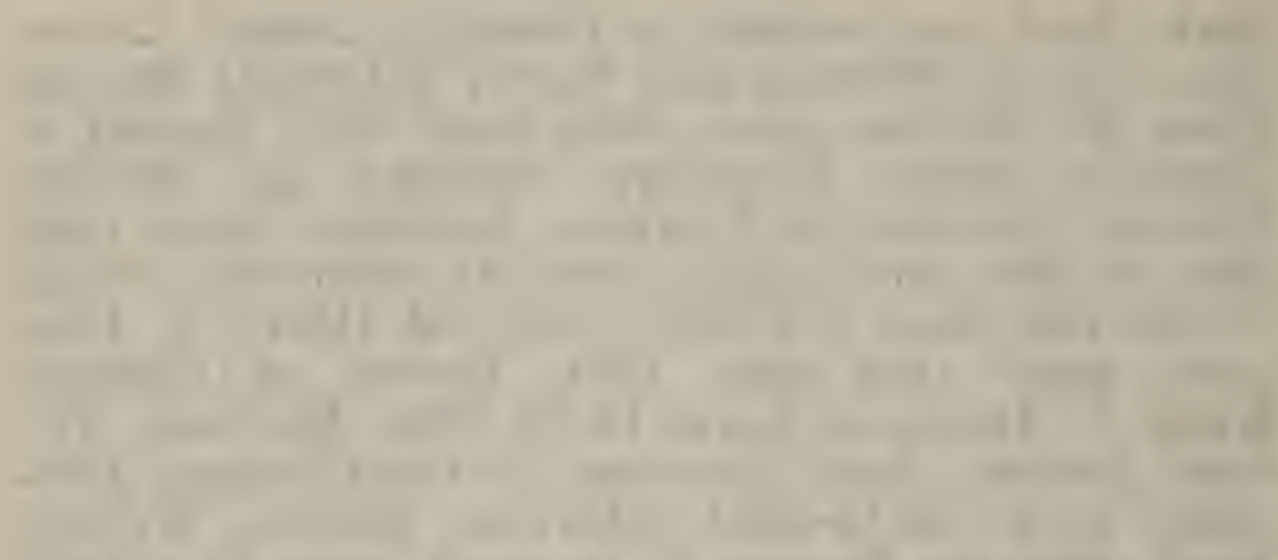

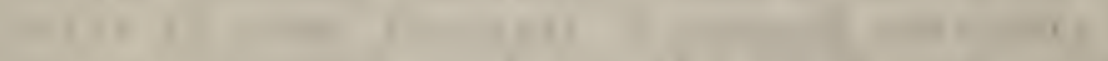

(10)

a

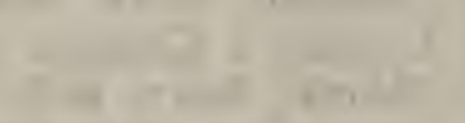

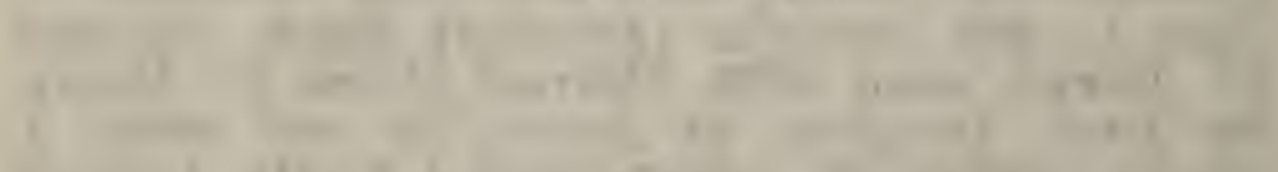

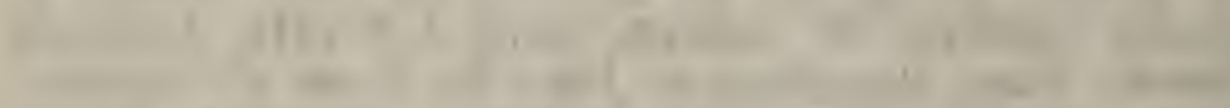

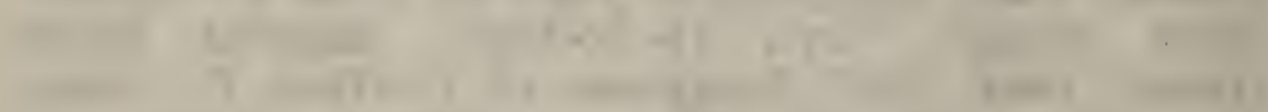
100

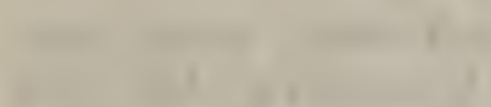

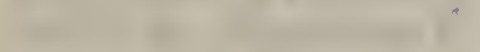

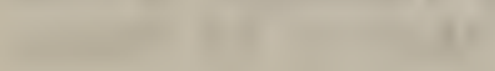


and Marvella (Johnson) Marsh. Willard N. Joseph, born 1-15-1906. Mirried Ruth Stayton, born 1127-1905. Daughter of Arthur and Anna Stayton. One son Kenneth A. Joseph, born 1951. Edna M. Joseph, died in infancy. Elsie M. Joseph, boria 12-12-1914. Married Carlton Smith, born 8-4-1913. Son of Chalton and Eva J. Smith. Four children. Ethel E. Joseph, born 2-7-1908. Married Norman J. McIlvaine, born 8-2-1913. Son of Harvey J. and Sara (Legates) Mcllvaine. One child, Allen J. Mellvaine.

Edgar F. Joseph married Anna Mcllvaine, daughter of Winter and Mary McIlvaine. One son, J. Edgar Joseph married Ava Truitt.

Mary Emma Joseph, born 1892, died 1961. Buried St. John's Methodist Church Cemetery. Married 12-19-1915 Joseph Walls, born 1891. Son of Shepard and Louise E. (Truitt) Walls. One son, Chester J. Walls, born 1919. Married 1940 Margaret Downs, born 1923. Daughter of Silas and Lena (Wilson) Downs.

Bessic Lee Joseph, born 4-2-1888. Married Clarence Johnson, born 6-7-1876, died 9-12-1961. Buried Coolspring Presbyterian Cemetery. Son of Thomas and Hetty (Perry) Johnson. Edwin Johnson, born 10-25-1913. Married Clara Wharton daughter of Wm. L. and Larrina H. (Mears) Wharton. Six children. Ralph Johnson, born 1916, died 1917. Calvin M. Johnson, born 1918. Married 1st. Charlotta A. Donovan, deceased. Married 2d. Beatrice MacDonald. No issue. All information reccived from the Walls Book complied by Mrs. Elsie Robinson.

The following information received from Mrs. Louder Mitchell and the grave markers in St. John's Cemetery. Jonathan Joseph married Mary Johnson, died 1887, 74 years. They are believed? to be buried at St. John's Methodist Cemetery. 

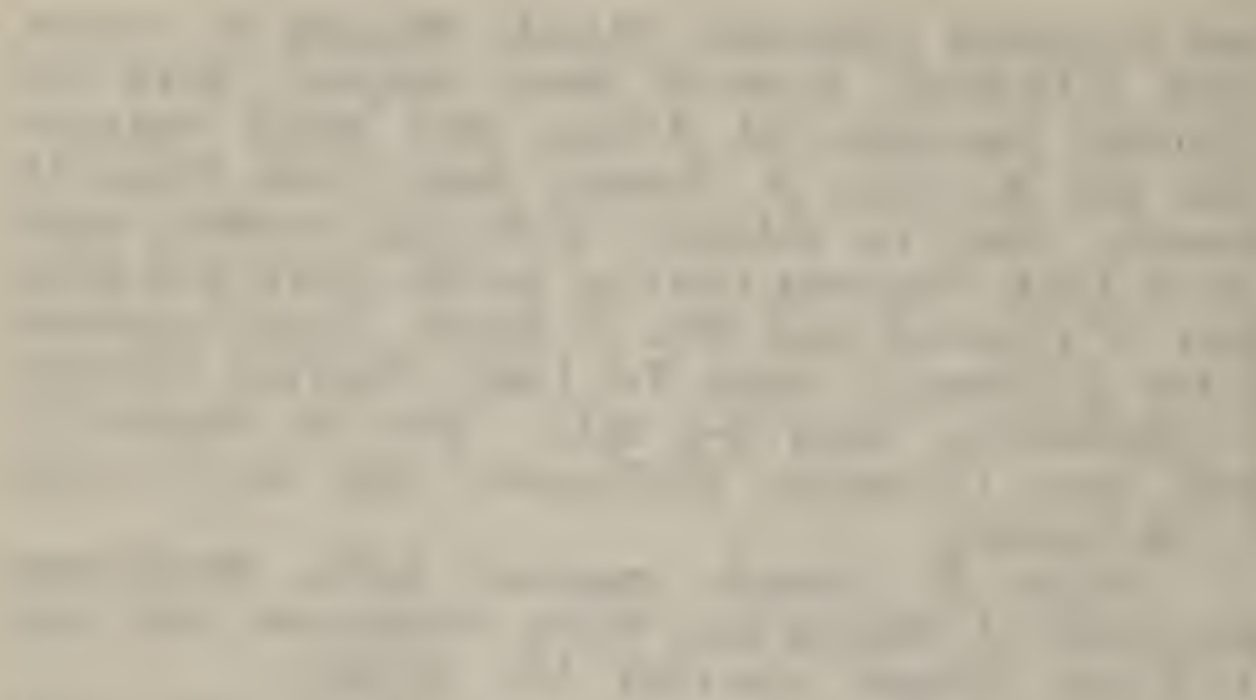

1

$\sqrt{2}$

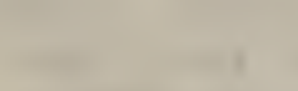

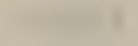

(1)
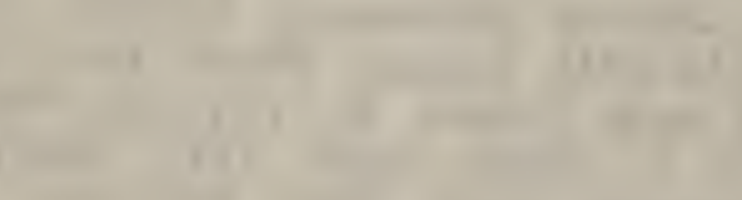

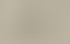

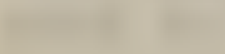

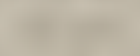

(1)

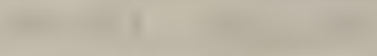

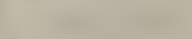

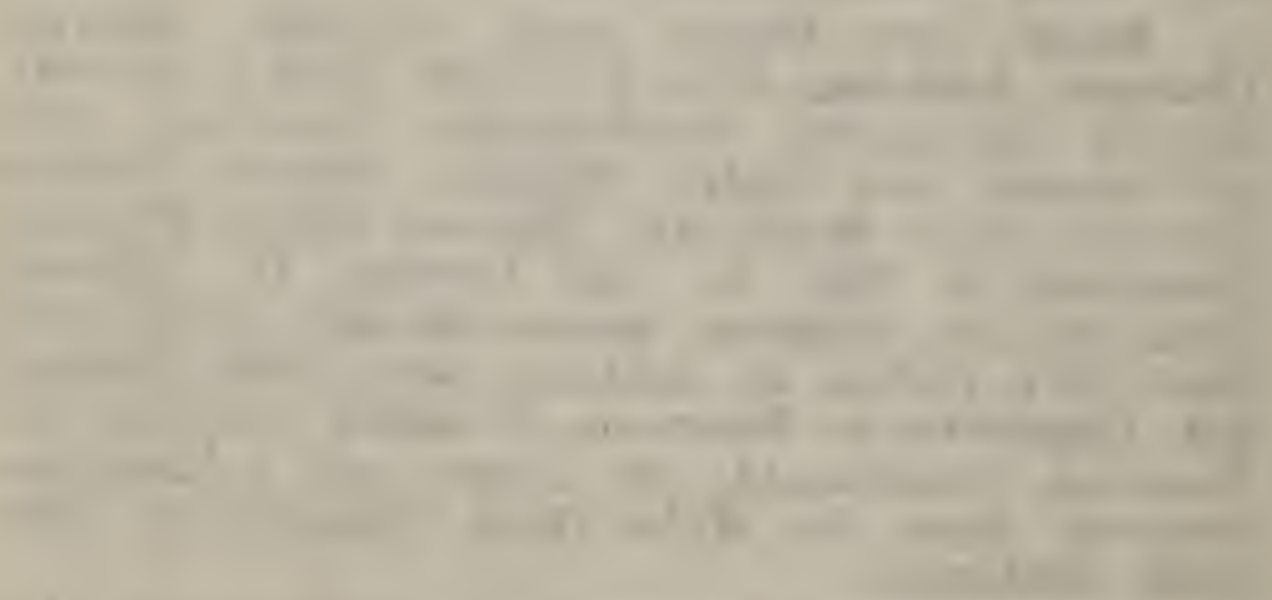

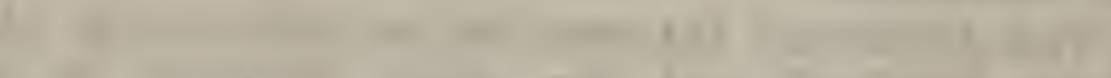

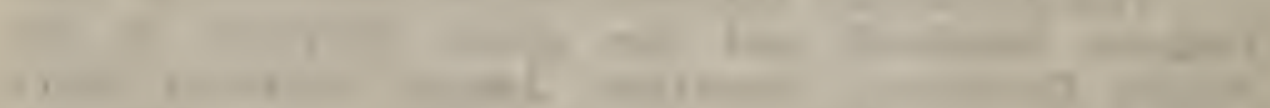

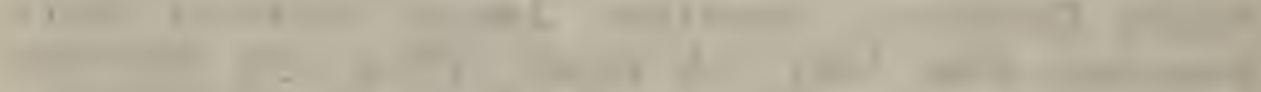
(

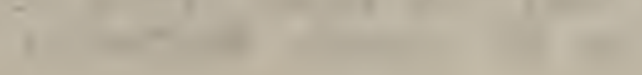

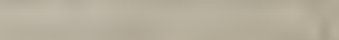


Rufus A. Joseph, born 8-4-1846, died 10-27-1919. Murried Arcodia Robinson, bcrn 9-15-1868, died 3-30-1952. Both buried St. John's Cemetery. Daughter of Parker and Mary Ann (Simpler) Tobinson. George A. Joseph, 1884 - 1942. Married Bessie L. Joseph, 1884 - 1957. Daughter of Walter andi Elizabeth Joseph. Both are buried in Lewes Bethel Methodist Cemetery. One daughter Edna Joseph married Irving Vienat. Ernest E. Joseph, burn -15-1895, died 11-25-194t. Buried St. John's Cemetery. Unmarried. Charles R. Joseph, born 18:32, ciled 1957. Buried St. John's Methodist Church Cemetery. Married Flossie Daniels, born 1901, died 1951. Buried Sand Hill Church Cemetery. Daughter of Elias M. and Margaret E. (Short) Daniels. Four sons, Charles R. Joseph, Jr.; Leo Joseyh; Layton Joseph; Alfred N. Joseph. Mary K. Joșeph, born 11-18-1885, died 6-9-1960. Buried St. John's Cemetery. Married David A. Steward, born 6-21-1882. Son of David A. "Steward, Sr. No issue. Paynter Joseph married Mary One son, Alton R. Joseph, born 3-22-1929. Married Hazel Johnson, born 3-16-1937. Three children, Alton E., born 10-22-1953; David Lee, born 6-231955; Linda L., born 3-3-1957. Horace Joseph. Unmarried. John Joseph, born 1888, died 8-181961. Buried in Wilmington, Delaware. Married 1st. Margaret ——, horn 1897, died 1932. Buried St. John's Cemetery near Georgetown. One son, Harry P. Joseph married Betty -. John married 2d. Yirginia Reynolds. Deceased. Buried in Wilmington. Iaura Joseph married Louder W. Mitchell, Sr. Daughter of Frank and Katie Mitchell Oue son, Louder W. Mitchell, Jr. married Jane Thomas, daughter of $\mathrm{Mr}$. and Mrs. Thomas of Philadelphia. Four children. Wm. Mitchell; Lee J. Mitchell: Robert Mitchell; Jerry Lymn, born 6-19-1963. E. Paynter Joseph buried St. John's Church Cemetery near Georgetown. 


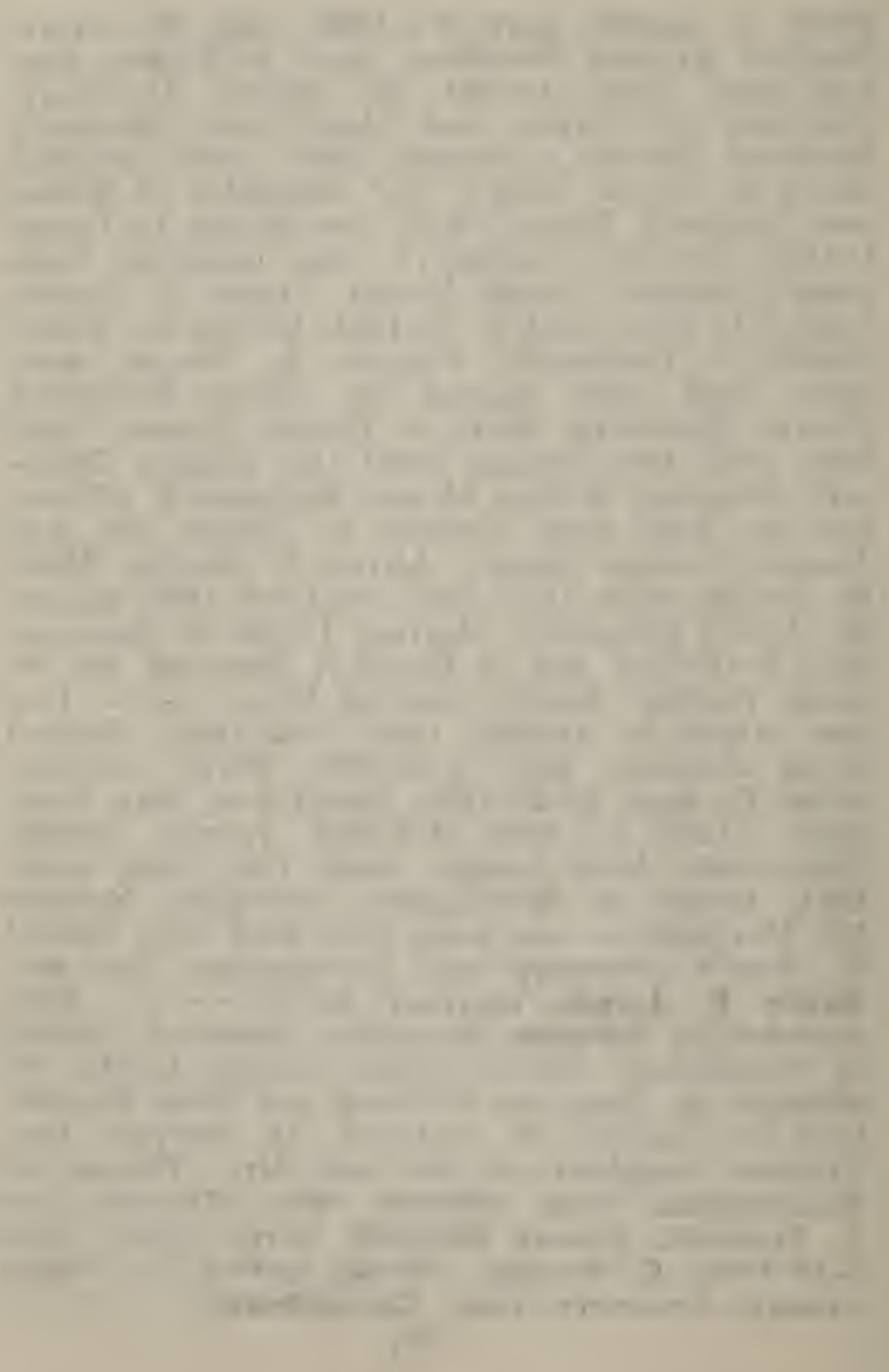


Mary Elizabeth Joseph, born 1843, died 1898. Married William E. Mlessick, died 11-1-1909, 62 years. Both are buried at St. John's Cemetery. Clemmie Messick, born 1884, died 9-15-1963. Married Polk Carey, born 1880, died 1961. Both buried Lewes Bethel Methodist Cemetery. Son of George and Virginia (Hollaway) Carey. 'Two children. One died in infancy. Buried St. John's Cemetery. George R. Carey married Pauline Grives daughter of Wm. and Frances (Green) Graves. Two sons G. Roland Graves Carey, Paul Graves Carey. We are not able to find any intormation about the following children of Hezekiah Joseph. Ebeneza Joseph, Hezekiah Joseph, Jr., George W. Joseph, Ann Joseph. Elizabeth Joseph. She is believed to have married a Walls.

The fourth son of Jeremiah. The Will of Joanthan Joseph Yeoman of Sussex County, Delaware, Delaware Inclian River H. Dated 3-28-1831, probated 5-11-1835 in Will Book H. 8, page 292, inentioned two daughters, Nancy Joseph, wife of Wm. Wilson, and Elizabeth Joseph, wife of Zackariah Tam. mentioned a niece, Frances Joseph, He did not mention a wife, she was probably cleceased. Witnesses; Peter Walls, George W. and Jose Joseph. The above mentioned Nancy Wilson had a son Jonathan J. Wilson who married Amy Joseph. This is all we (L.V.H.) have found about this family. The 8 th child of Jeremiah Joseph. Zackariah Joseph, born 1766, died 11-3-18+2. Married Mary Walls, born 1767, died 1837. Sarah T. Joseph, born 3-20-1789, died in infancy. Eli $T$, Joseph born 2-5-1800. Sarah Joseph born 5-19. 1303. Wrn. C. Joseph, 9-7-1791 married

It is believed that this is Wm. C. Joseph the father of Wm. C. Joseph who married 1838 1st. Amy Simpler, daughter of Peter and Lurana (Bennum) Simpler. 'I'wo children. Casandra Josi- 


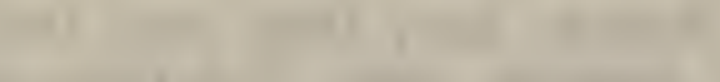

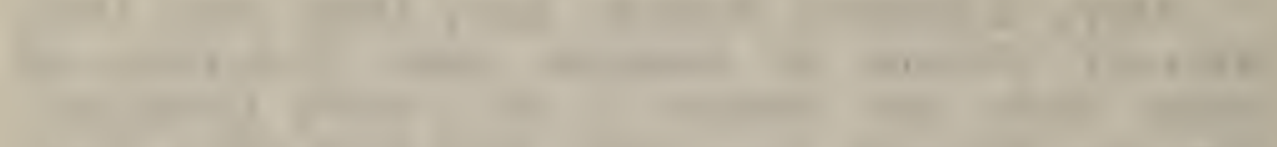

1. and

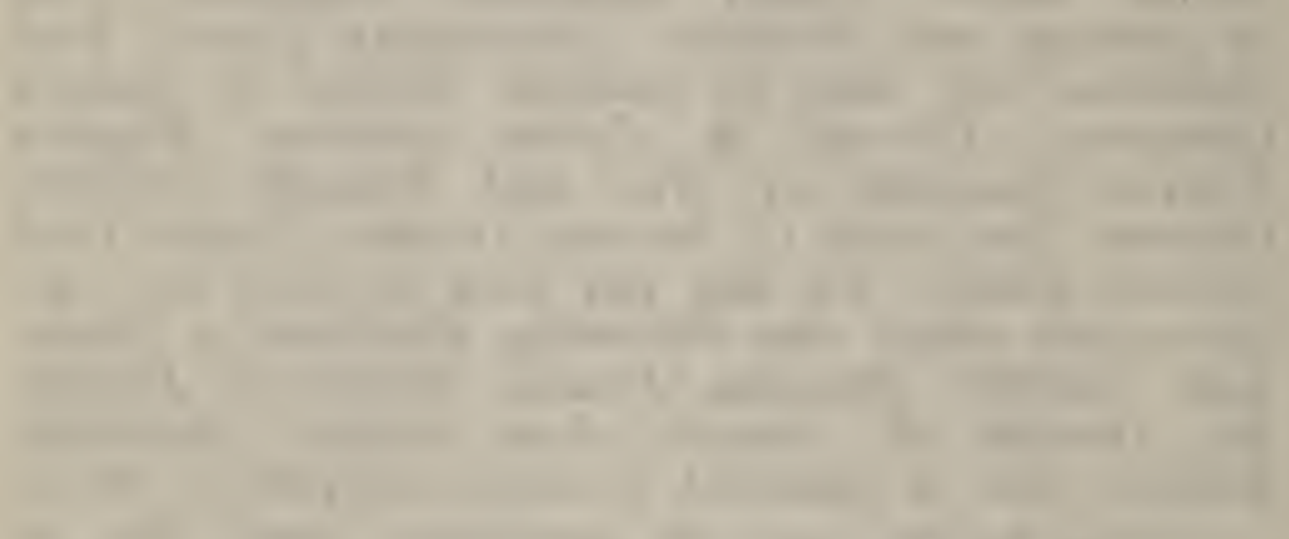

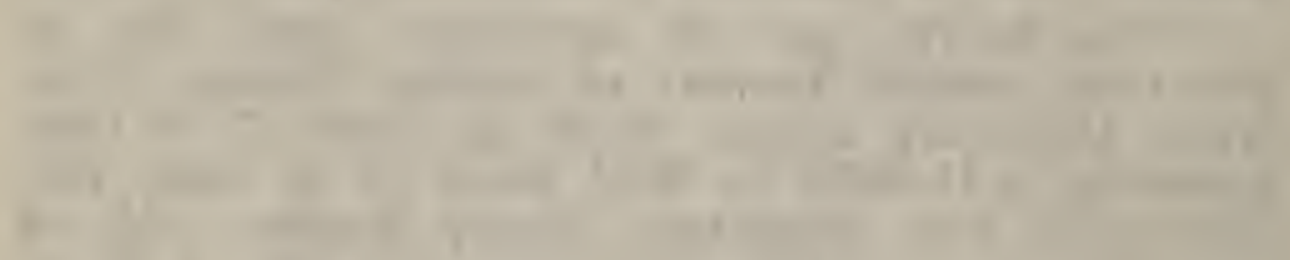

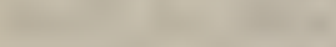

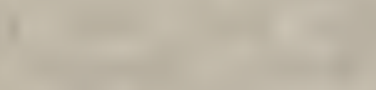

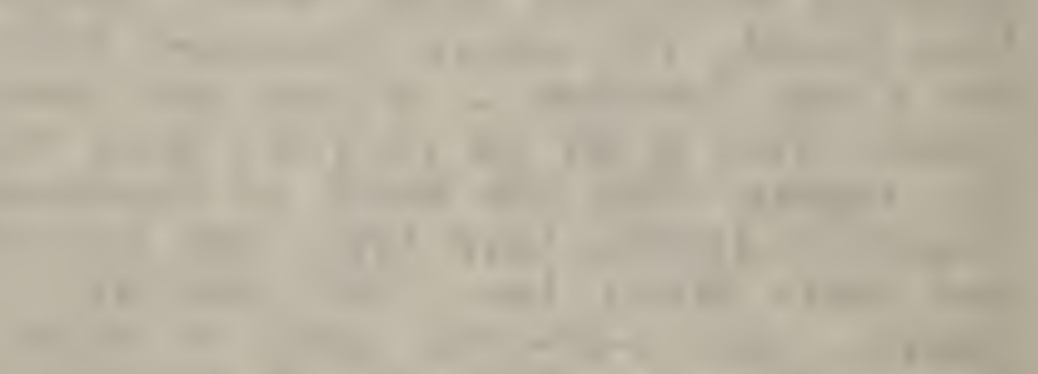

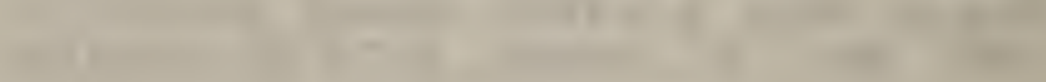

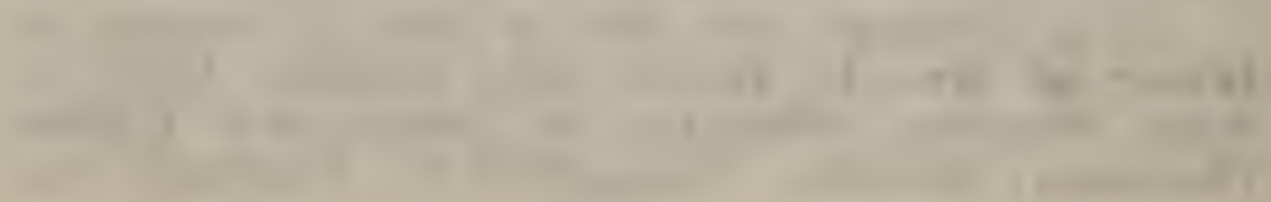


eph. This iniormation received from the Walls Book. Louisea Joseph married Harry Orth. One claughter Carrie Orth.

Wiliam C. Joseph, born 1818, died 5-8-1891. Married 2d. Hettie R. Green, born 9-17-1829, died 8-31-1904. Daughter of John and Lydia (Rust) Green. Both are buried St. John's Methodist Cemetery. Ella C. Joseph, born 3-10-1849, died 1-311920. Married Robert M. Joseph, born 5-3-1840, died 3-2-1915. Buth are buried St. John's Cemetery near Georgetown. Hannah Joseph, born. 1869, clicd 1947. Married Columbus Rogers, born 1870, riied 1943. Both are buried in Union Cemetery, Georgetown. Ernest M. Rogers, born 1890. Married 1st. Mattie Marvil, born 1890, died 195.0. Buried Union Cemetery Georgetown. Daughter of Gardner all Annie M. (Calhoun) Marvil. He married 2d. Nary Webb, daughter of Wm. and Clora Webb. Luster H. Rogers, born 10-19-1891, died 2-3-1960. Buried Union Cemetery, Georgetown. Married Flizabeth Fowler, born 4-22-1894. Daughter of Charles R. and Josephine (Dickerson) Fowler. Florence Rogers married John King, son of Peter King. One daughter Beatrice King. Married. 2 d. Robert K. Wyatt. One daughter, Sharon. Norman M. Rogers, born 9-14-1902. Married Ethel. Savage, born 8-4-1900. Daughter of Reuben L. and Lizzie (Messick) Savage. Four children. Catherine Rogers, burn 6-11-1921 married Harry Zerby, born 1918, died 1962. Buried Union Cemetery, Georgetown. One son Jefiery Zerby born 2-6-1943. Alton L. Rugers, born 4-18-1924 married Dorothy Coulter, daughter of Edgar B. and Florence (Rogers) Coulter. One child Joyce Ann Rogers, born and died 1949. Buried Union Cemetrey, Georgetown. Robert D.Rogers, born 6-29-1927 married Lola Calloway. Robert D. Jr., born 11-4-1950. Karen, born 1956. Kenneth E. Rogers married Dot Mari- 


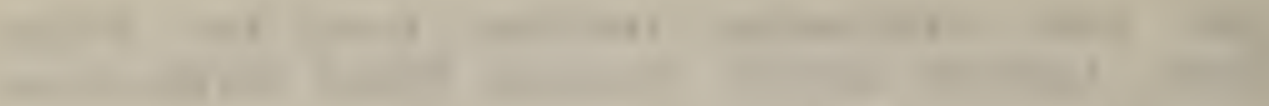

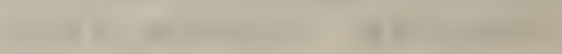

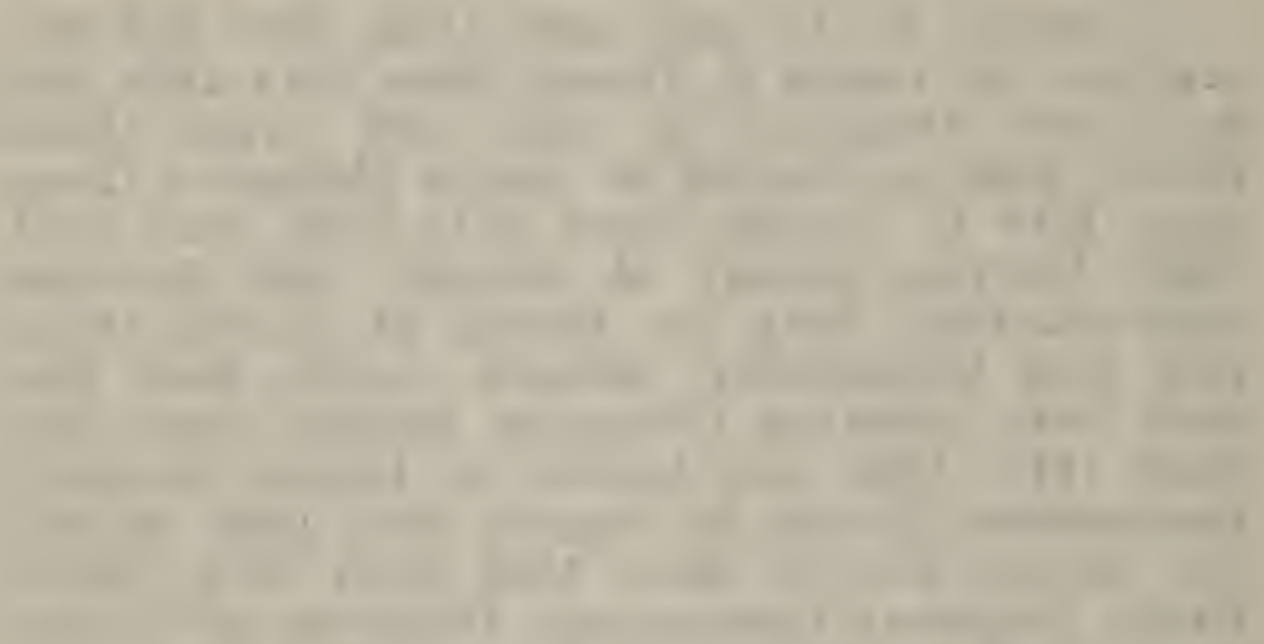

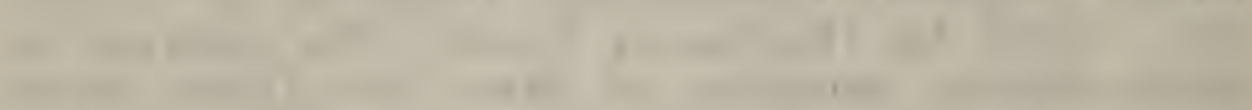
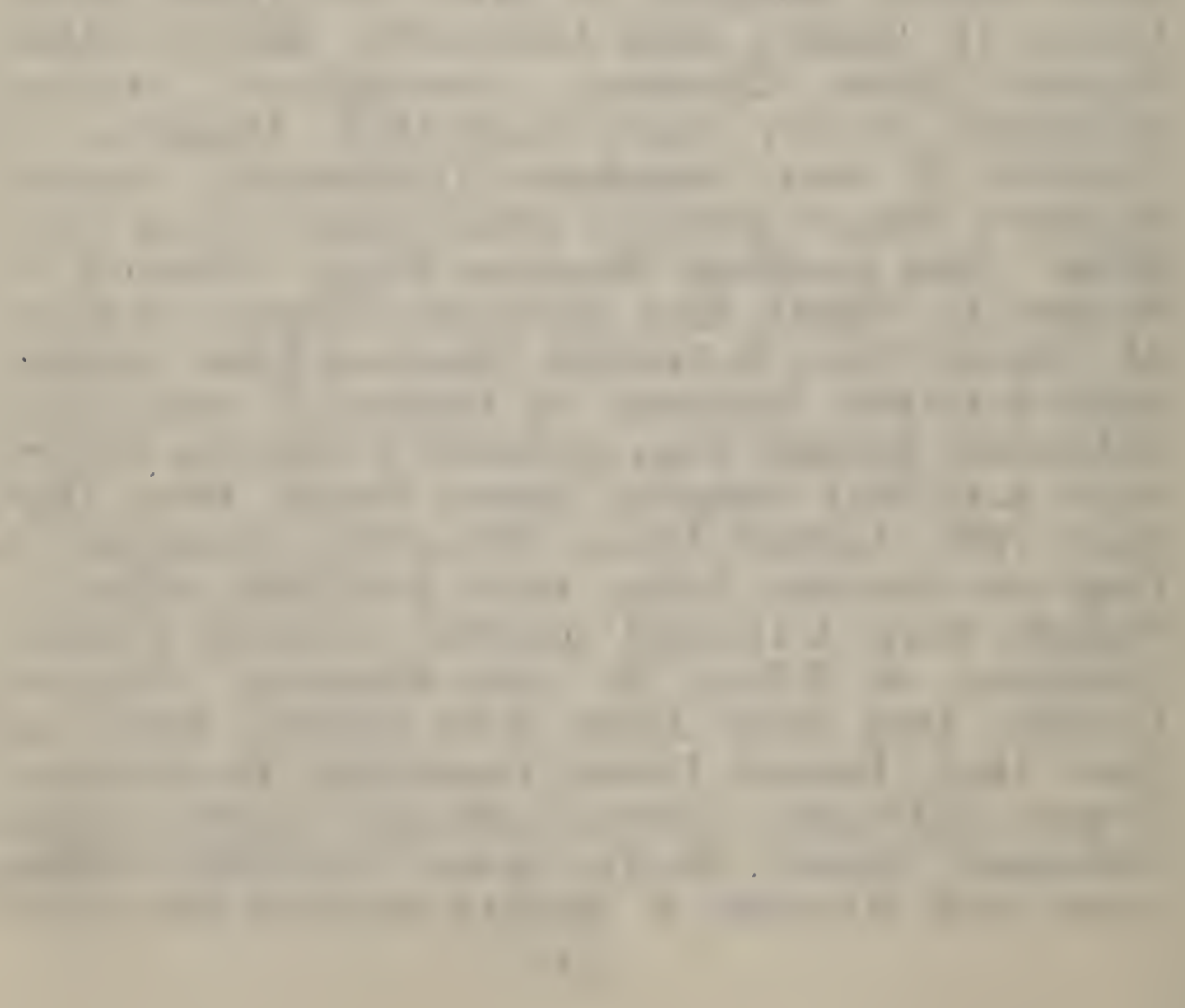
ner. Donna R., 1-8-53; Bruce R. 1958; Steven R., received from Mrs. Norman Rogers of Georgetown, Delaware.

William E. Joseph, born 1871, died 1961. Married Addie L. Rogers, born 1878, died 1954. Both are buried Union Cemetery, Georgetown. Daughter of Jewel and Sarah (Morris) Rogers. Hattie Joseph, born 1898, died 1950. Buried Union Cemetery, Georgetown. Married - Grirfith. Edith Joseph, born 1900 married Norman Baker No issue. Cecil W. Joseph, born 1906.

Charles.R. Joseph, born 1873, died 1943, married Anna M. Mears, born 1877, died 1952. Buried Union Cemetery, Georgetown. Daughter of Annamious and Sabra (Elliott) Mears. Ethel Joseph married Rufus Wilson, son of Nath. and Sallie (Torbert) Wilson. A daughter Marguerite Wilson married Alves. Howard A. Joseph, born 1903, died 1931. Buried Union Cemetery, Georgetown. Married Una Stevens. One son, Charles H. Joseph, born 11-10-1927, died 1954 buried Union Cemetery, Georgetown, married Sallie Amn Short, daughter of Isaacs B. and Ula Short. Two sons, Paul Richard, Jr., 10-31-1952-8-16-1953 Howard Joseph.

Harry O. Joseph, born 1874, died 1951 married 1st. Nancy C. Marvel, born 1879; died 1907. Both buried Union Cemetery, Georgetown. Armenia M. Joseph, born 1-31-1897 married John Robert Wilson, born 6-29-1890, died 1943. Buried Uion Cemetery Georgetown. Son of Theodore E. and Orphla J. Wilson. Two sons, Williain H. Wilson, deid age 6 weeks, James R. Wilson, born 8-4-1914. Married Jaunita A. Chase, born 9-22-1923. 'Three children, Lanya Diane, 6-6-1949; Frances, 6-101951; Candy Lana, 2-17-1905. Floyd E. Joseph, born 2-17-1905, died 8-14-1949. Harry O. Jcseph married 2d. Eugenia Coffin, born 1885, daughter 
(1)

1.

$\sqrt{3}$

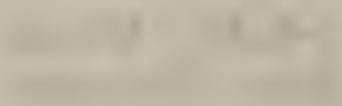

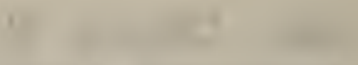

-

$-$

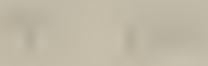

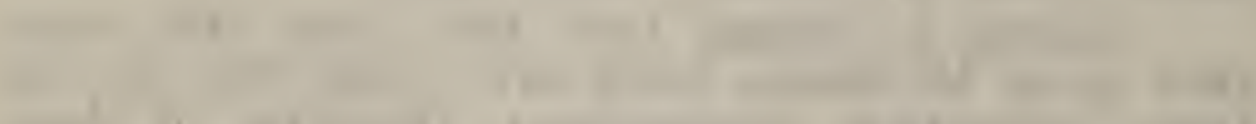

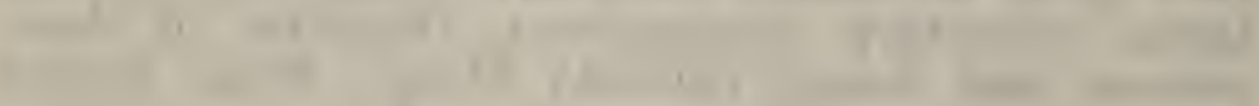

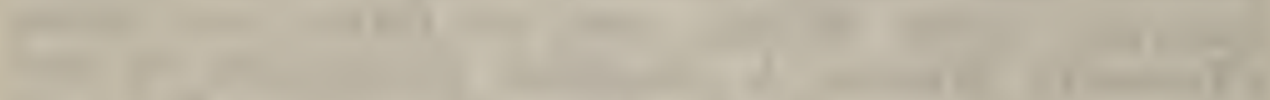

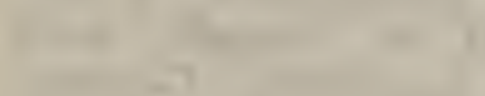

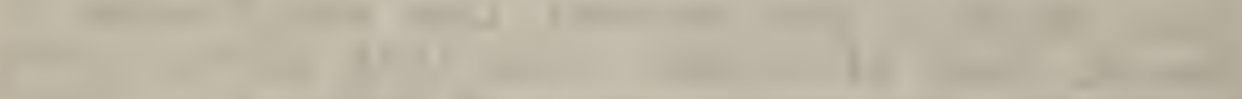

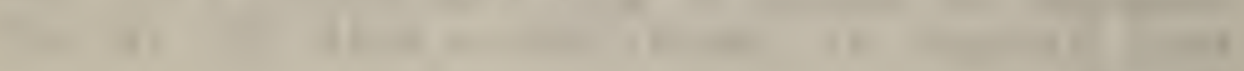
aty

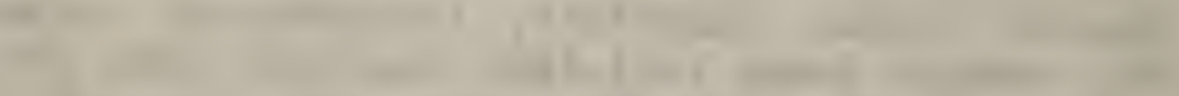
1) 
of Thomas P. and Maggie J. (Messick) Coffin, Two children, Mildred Joseph, born 6-6-1911, died 5-20-1955, buried Union Cemetery, Georgetown, married Charles P. Hawkins, Sr., son of Thomas $P$. and Dora B. Hawkins. Leon P. Hawkins, born 11-11-1926 married Norma V. Johnson, born 725-1y28. Six children, Leon Paul, 12-8-1951; Charles K., 1-18-1953; Michael Hawkins, 6-24-1955; Terrance Hawkins, 9-21-1957; David Hawlins, 1211-1959; Craig Hawkins, 7-25-1961. Charles P. Hawkins, Jr., 12-23-1930 married Charlotta A. Ly'nch, daughter of James Lynch. Four children, Diane E., 3-11-1951; Gary P., 4-5-1952; Deborah Y., 11-11-1955; Kenneth C., 5-4-1960. Pierce Hawkins, born 1928, died 1935. Buried Union Cemetery, Georgetown. Frank Juseph married - One son. The above data lecieved from Mrs. Armenia Wilson.

Carrie H. Joseph, born 1878, died 1939 married Frank P. Rogers, born 1878, died 1933. Both are buried at Providence Church Cemetery. Hoyt and Horace were twins. Hoyt J. Rogers born 325-1901, died 1946, buried Union Cemetery, married Cora M. Calloway, born 1901, daughter of Wm. Calloway. Hoyt J. Rogers, Jr., 6-13-1921 married Janet E. Hastings, born 1-11-1922. Two children, Janice Karen, born 7-27-19-6; Susan Elaine, born 7-26-1949. Betty L. Rogers, born, 3-11921 married Joseph J. Thompson, Jr., born 3-191821, son of Joseph and Ethel (Scott) Thompson. One child, Diane Kay, born 8-24-1947. Horace J. Rogers, born 3-25-1901 married Minnie Hudson, born 4-14-1899. One daughter Margaret Rogers, born 1928 married Charles Van Auken. Two daughters, Susan E., born 1956; Sandra E., born 1960. Robert P. Roggers, born 1902, died 1925, buried Mechanics Cemetery, Millsboro,married Flora Carmean. No issue, Daughter of Joseph F. and Anna M. (Gib- 

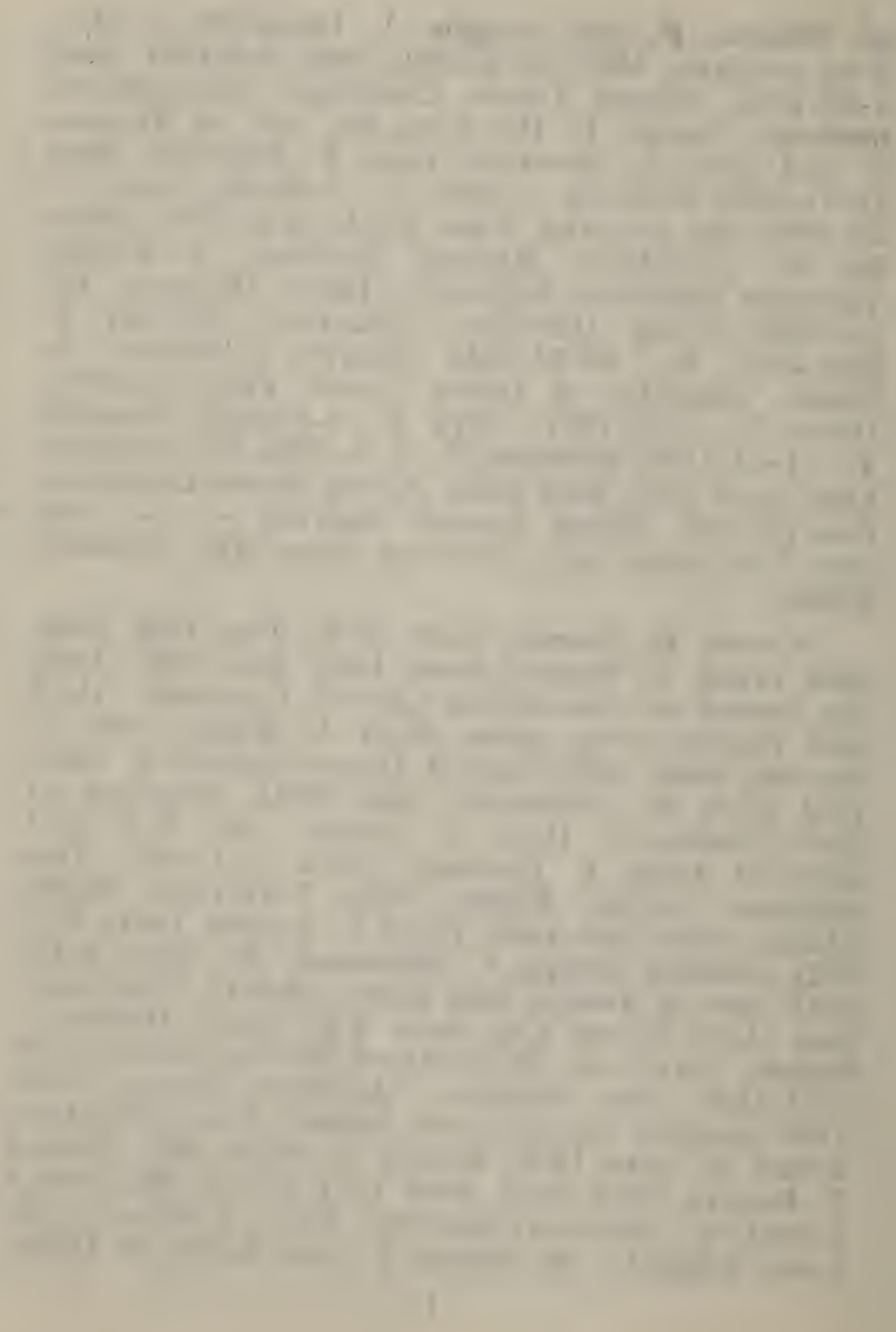
bons) Carmean. Delema Rogers married Thomas H. Rogers, son of Wilmore Rogers. One daughter, Hattie Rogers, born 11-11-1915 married Leroy H. Weatherly. One son Leroy Weatherly married Patsey Howard. One daughter, Sharon Lee, born 10-25-62.

John H. Joseph, born 1879, died 1956 married Lottic Smith, born 1885, died 1955. Both buried Union Cemetery, Georgetown. Daughter of Hiram S. and Anna (Wilkins) Smith. Carlton Joseph, born 1918, died 1952, buried in the same place, married Mary Josephine. Two children, Charlotte E.: Carlton E. Joseph.

Icla Joseph, born 2-23-1886, married Clarence Thompson. 'Two sons. Both live in Wilmington. Clarence Thompson, Jr., born 2-14-1918 inarried Beatrice Harriett. Three children. Paul Thompson, born 5-7-1927 married Jackeline Steward. Three children. This received from Mrs. Ida Thompson of Wilmington, Delaware.

Horace D. Joseph, born 1891, died 1944, muried Union Cemetery, Georgetown, married Estella M. Wilkins, born 1885, daughter of John H. and Comfort ('Taylor) Wilkins. Hannah Joseph, born 1127-1849, died 8-4-1890, buried St. John's Cemetery, married Alex Weeks. No issue.

Charles Hill Joseph, born 11-21-1851, died 10-21-1919 married Sarah E. Hudson, born 1850, died 1927. Both luried St. John's Cemetery, Georgetown. Daughter of Levin and Margaret Hudson. Daniel D. Joseph, died in infancy. Buried St. John's Cemetery. L. May' Joseph, born 7-71893 married Lawrence A. Hitchens, born 1-251899, died 1-13-1956, son of Daniel T. and Mary (Messick) Hitchens. Fmma. Pearl Hitchens, born 9-28-1921, married-5-14-1938, Walter Elwood Franklin, boin 6-28-1918. Two children. Son of John 'T. and Louise Flanklin. Donald Paul Frank- 
lin, born 10-ई̃-1941 married Kathy Roberts. One child, Michael Jay Franklin, born 5-23-1963. Daughter of Woodrow Ruberts and wife Edna. Joan Ann Franklin, born 10-19-1947. Doris Elva Hitchens, born 8-19-1925 married 1943 Clarence Johnson, Jr., born 1923, died 7-7-1962. Buried Odd Fellows Cemetery, Milton. Son of Clarenco and Rebecca (Morris) Johnson, Sr. Seven children. Irene Ann Johnson, born 3-14-1944 married Wayne Edward Rust son of William G. and Myrtle (Massey') Rust. One child, Dwayne Rust, born 11-151952. Richard J.; Robert J.; Lou J.; Clarence J.; Theo. J.; Barbara J. Lawrence Vernon Hitchens, born $6-21-1934$.

Amy Hester Joseph, born 5-23-1853, died 914-1925, married Joln H. Dodd, born 3-4-1851, died 9-14-1922. Both are buried St. John's Cemetery. Son of Peter P. and Mary E. (Palmore) Dodd.

Jennie Dodd, Lorn 1880, died 1942, married Harry Veasey, born 1879, died 1922, son of Nath 'I. anci Harriet E. (Virden) Veasey. Nath. C. Veascy married Ethel Betts. Four children, Donald C. Veasey: Dallas Veasey; Ollie Lee Veasey married Delbert Scott. Two children. James Mason Veasey. Margaret Veasey, born 1911, died 1936 married Joseph Hunter, born 1911, died 1952. Both buried St. George's Chapel Cemetery. Son of Wilmer and Mary (Hill) Hunter: Lettie G. Hunter married Warren Pettyjohn son of Otwell and Annie (Warren) Pettyjohn. One daughter, Betty Gae. Josephine Hunter married J. Roy Muir, son of Jennings and Madlyn Muir. Three children, Julia Ann - Jan Lynn, born 2-20-1960; Steven, born 2-22-1957. Betty Ann Hunter, married 9-12-1953 George B. Donaway. One son Bart. Hammond Veasey, born 8-2-1914, died 7-15-1956. Buried Harbeson. Glive B. Veasey, born 6-25-1913 


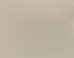

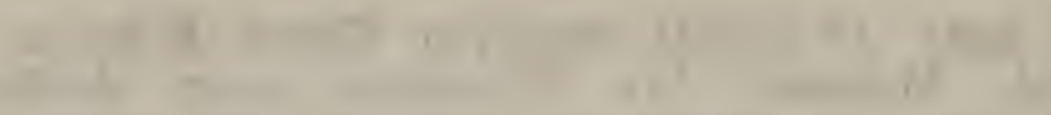

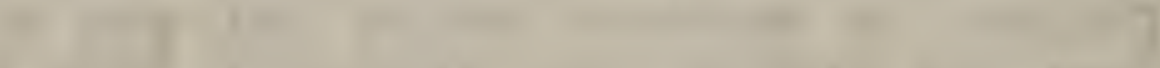

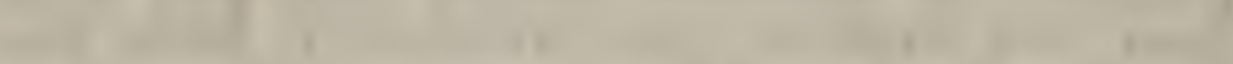

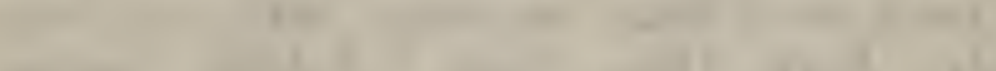

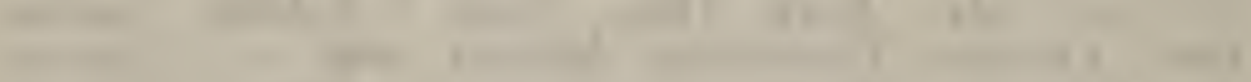

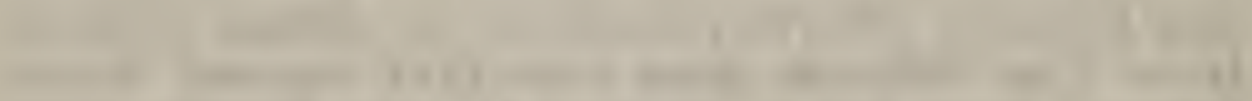
-

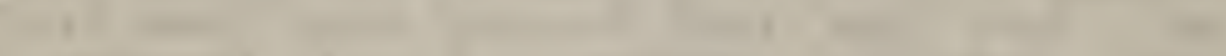

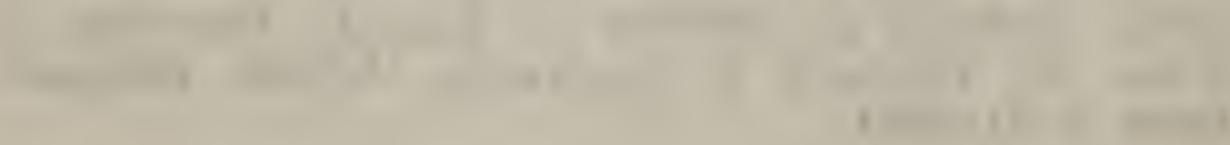

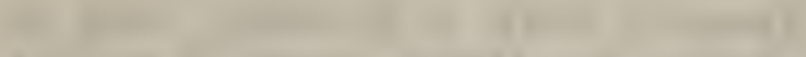

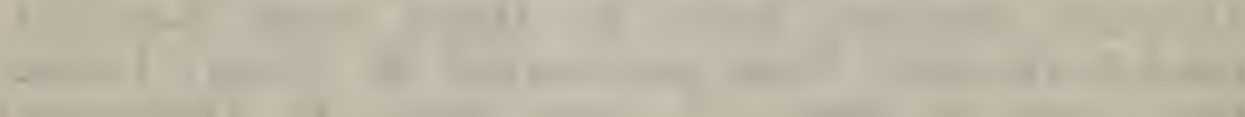

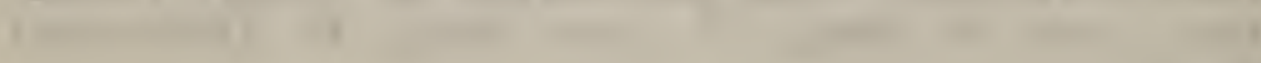

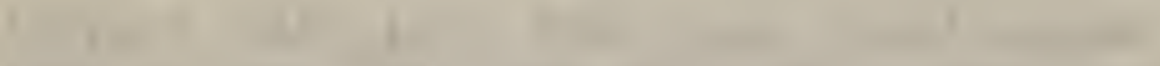

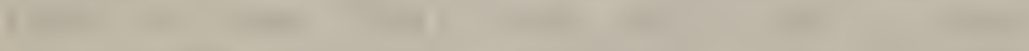

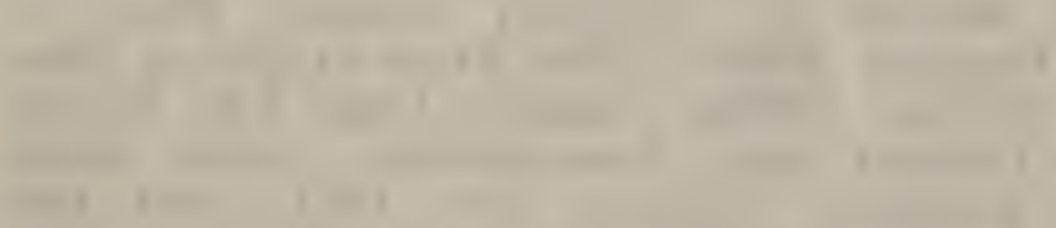

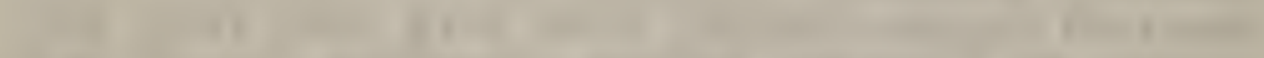
(1)

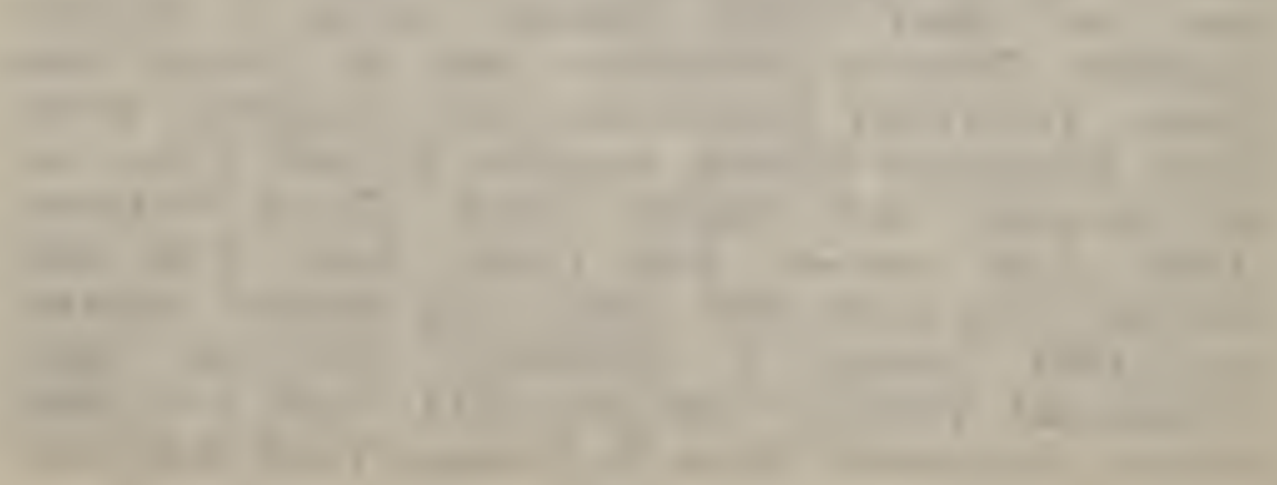


married Arthur L. Pittard, son of George and Eva (Burton) Pittard, Sr. Lelia M. Veasey, born 2-10-1920 married Thomas Pittard, son of Geo. and Eva (Burton) Pittard. One daughter Janet L. Pittand married 10-26-1963 Harry Hobson Isaacs, son of Harry Isaacs, Jr: Margaret Veasey died in infancy. Buried Harbeson. An unnamed child buried at St. John's Cemetery.

Goldia W. Dodd buried at St. John's Cemetery married Mayme Simpler. This family is found under the Joseph, Walls and Simpler Geneology in this book. John Hollis Dodd, born 1891, died 1952. married Blanche N. Marvel, born 1892, died 1954. Both buried St. John's Cemetery. Daughter of Edgar T. Marvel. W. Conwell Dodd, born 8-211910. died 7-__ 1951. Buried Beaver Dam Cemetery, married Pearl L. Koepple, born 1908, daughter of Guston H. and Priscilla E. (Calhoun) Koepple. Four children, Donald Dodd; Richard H. Dotd; Harold Dodd; Shirley Ann Dodd, born 1939. died 1940. Buried Harbeson Cemetery. 2d. daughter of John H. and Blanch Dodd. Helen Dodd married Wm. Wolfe. Virginia Dodd married Henry Ellingsworth. Blanch $H$. Dodd, born 4-23-1895 married Clayton Cordrey, born 188?, died 1955. Buried Mechanics Cemetery, Millsboro, son of Job and Nancy (Baker) Cordrey. One daughter, Elsie Cordrey, born 11-2-1921.

William M. Joseph, born 1854 , died 1933 married Zipporah Jefferson, born 1855, died 1935. Both buried Union Cemetery, Georgetown. Hoyt Joseph, born 1881, died 1929, buried Union Cenetery, Georgetown, married 2-25-1903 Lizzie R. Wilson, daughter of Jonathan and Caroline (Wilson) Wilson. One daughter, Myrtle H. Joseph, born 10-3-1907, married 11-26-1924 Raymond F. Jestice. Four children, Raymond F. Jestice, .Tr., born 1-11-1927, died 2-23-1936. Ruth E. Jestice, born 12-23-1927, married 6-27-1952 Donald Kelly

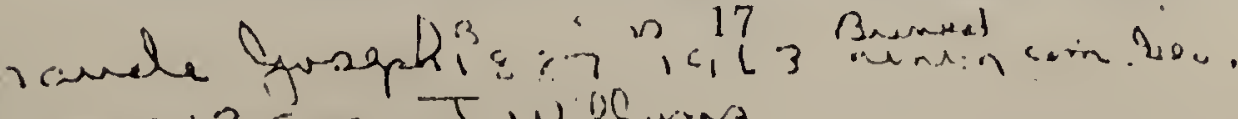




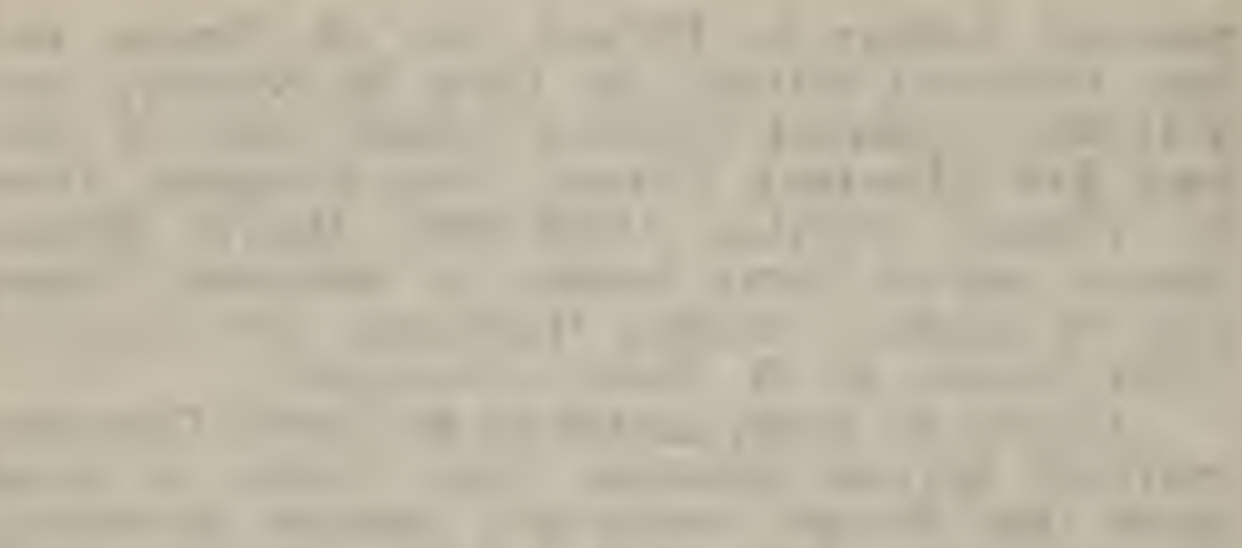

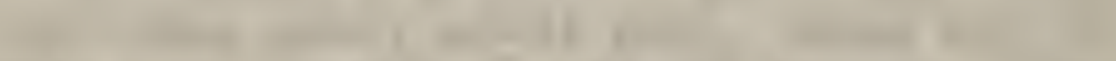
+

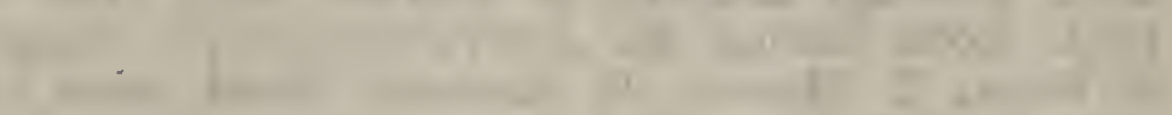
14

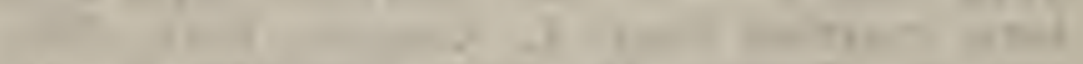

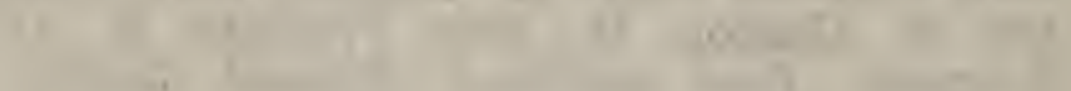

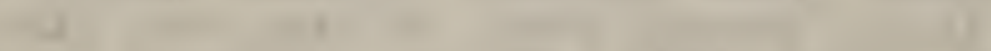
1

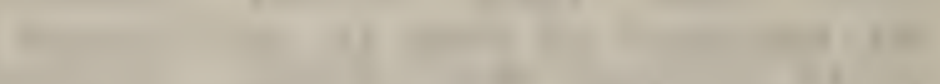

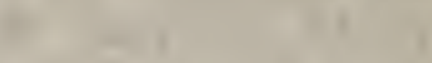

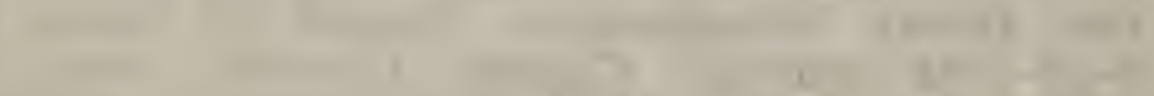
then

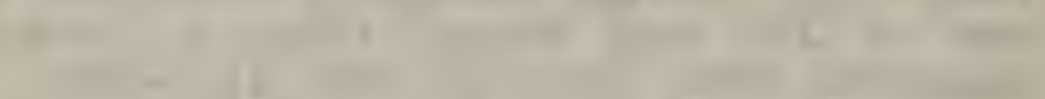

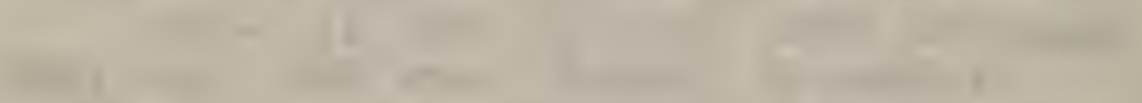

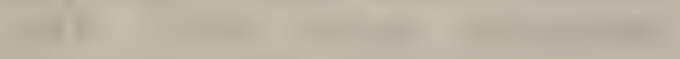

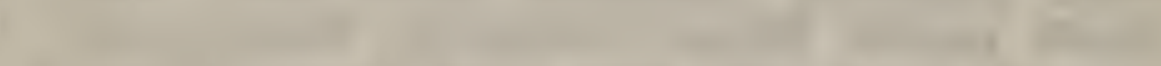

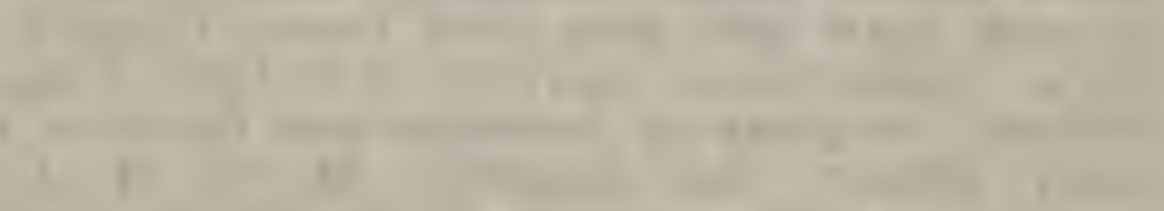

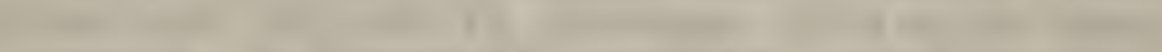

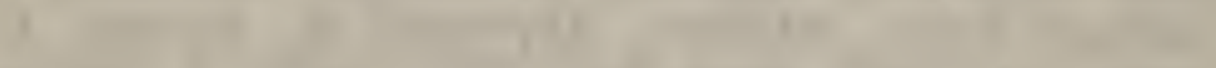
(1) 10 
Foster. One child, Lynn Kelley, born 9-1-1957. Lawronce Edward Jestice, born 7-20-1937, married 12-(i-1955, Betty Woodward. Three children, Susan Elizabeth Jestice, born 10-21-59; Lawrence E. Jestice, born 6-1-1961; Gail Ellen Jestice, born 11-19-1962. James Franklin Jestice, born 9-2-1940. The above information received from Mrs. Myrtho Jestice of Laurel, Delavare.

John D. Joseph, Sr., born 9-15-1856, died 27-192. married 1-1-1893 Jennie A. Rust born 1865, died 1940. Buth buried St. John's Methodist Church, Cemetery. Daughter of Peter W. and Mary (Hammond) Rust. Paul R. Joseph born 1221-1886, died 12-7-1959, married Addie E. Truitt, born 1886, died 1958. Both buried Gracelawn Cemetery, Wilmington. Daughter of James B. and Lavina (Downs) Truitt. Paul Joseph, Jr., born 1030-1918, died 9-24-1948. Buried the same place. John D. Joseph, Jr., born 5-5-1905 near Georgetown, Delaware, married Jerdie Lowe, born 6-11906, daughter of Minos Lowe. Lillian Joseph married Harry Lemmon. Two children, David Lemmon; Kathy Lemmon. Lorraine Joseph, born' 1933, died 9-28-1962, buried Riverview Cemetery, Wilmington, married Gerald Mackey. Three children, Linda Ann Mackey, born 1953; Doss Mackey, born 1957; Gerald P. Mackey, born 1955. Elizabeth Joseph, born 12-22-1910 near Georgetown, Delaware, married 6-18-1931 Howard O. Greene, born 1-16-1909, son of Rufus and Katie (Rogers) Richard R. Greene, born 1945. The above information was received from Mrs. Betty Greene. Greene. Two sons, Iloward O. Greene, born 1941; Richard R. Greene, born 1945.

Seth Edivard Joseph, born 1859, died 1884 '. Buried St. John's. Unmarried. Corneolius W. Joseph, born 1862, died 1943, married Ida J. Wilson, born 1863, died 1901. Both' buried St. 

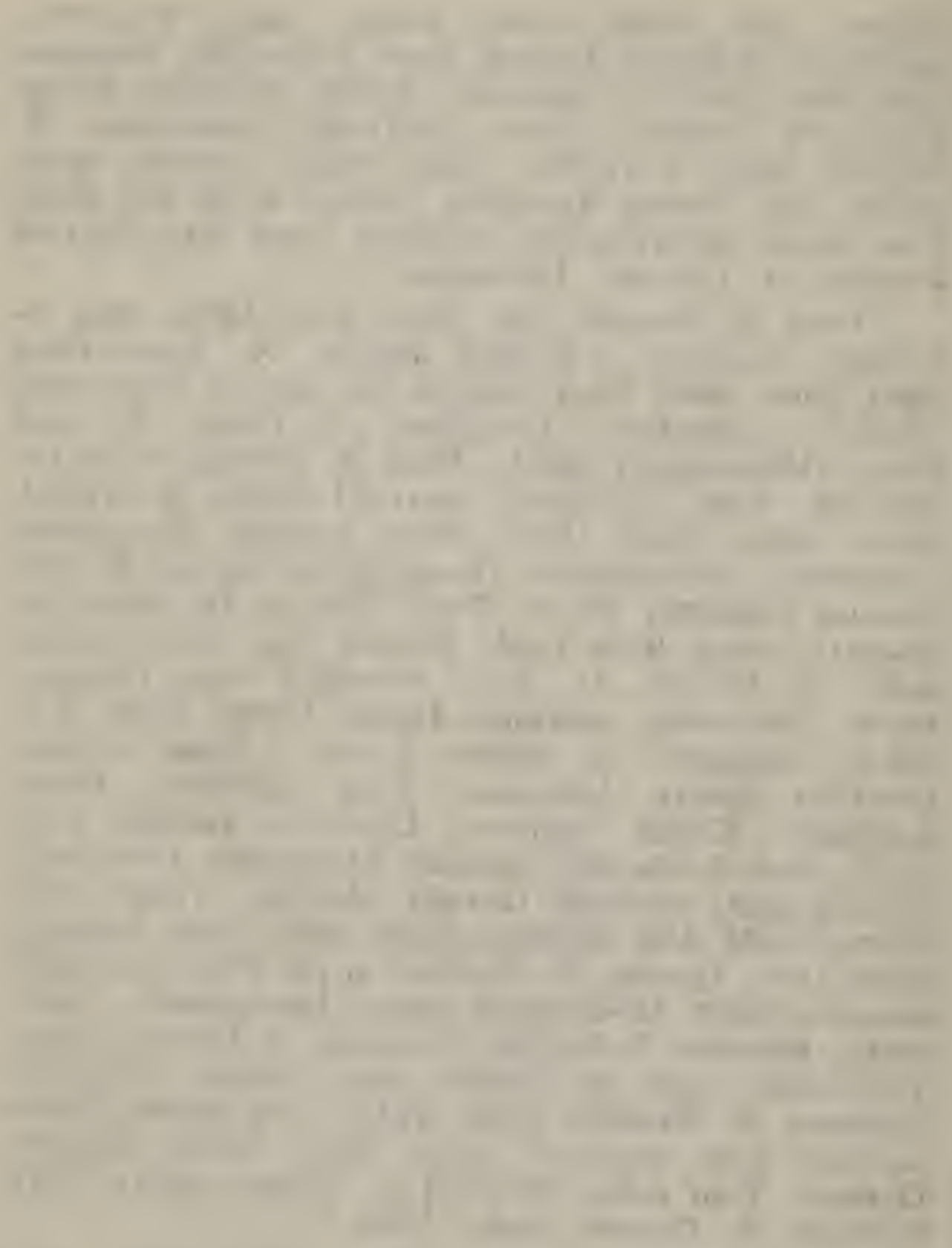

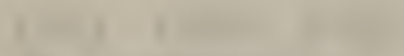

.

1 r n

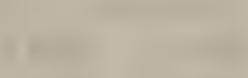

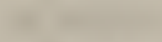
ar

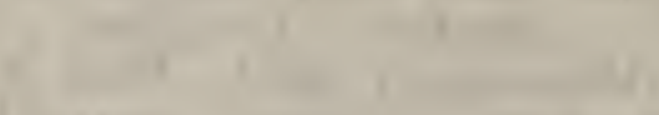


John's Church Cemetery. Edna Joseph married Clifford Wells. Both buried somewhere in Maryland. One son, Joseph Wells, married Florence - One son. Hattie Joseph married Fred Barr. Two daughter, Marie Barr, born 1913, died 1927. Burier Union Cemetery, Georgetown. Rachel Barr. Both were married. Correlius Joseph married $2 \mathrm{~d}$ : Lydia King. No issue. Daughter of Peter King. Elias H. Joseph, born 1864, died 1885. Buried St. John's Methodist Cemetery. Unmarried.

Anna Burke Joseph, born 1866, died 1951, married Robert P. Johnson born 1862 died 1927. Son of Albert C. and Elizabeth (Simpler) Johnson. Elizabeth was the sister of Wm. C. Joseph's 1st. wife, Amy. Jennie Pearl Johnson, born 1891, died 1961, buried St. John's Methodist Cemetery, married George W. Matthews, born 1895, son of Wingate E. and Anna Nathews. George Robert Matthews, born 1928, died 1952, buried St. John's Methodist Chunch Cemetery, married Betty Dill. Two children, George R., born 3-17-43; Bonnie, born 10-6-_. Crawford W. Matthews, born 3-261931, married, 1-11-1952 B. Jane Mundorf, born 10-12-1934, daughter of John B. and Mary Mundorf. Four children, Crawford W., Jr., born 10-211953; , David B., born 7-25-1955; Robie W., born 12-2-1957; Maryanne, born 3-29-1959. Edgar Johnson, born 2-25-1892, married Virginia Smullon. Two sons, Edward Johnson, died in the service of his country; Howard Johnson, dierl in infancy. Ralph R. Johnson, born 5-9-1894 married Lulu Short, born 3-25-1898, daughter of Peter M. and Sarah Short. Milton R. Johnson, born 8-20-1917, married Arla Hazelrigg, born 11-9-1918. 'Two daughters, Patricia M. J., born 12-8-1943; Janet M. J., born 12-18-1950. Pauline Johnson, born 125-1919, died, 10-22-1919, buried St. John's Cemetery. Helen Johnson, born 7-7-1921, married Al- 
fred Dickerson, divorced. Three children. Son of Clarence and Pearl Dickerson. Richard A. Dickerson, born 2-24-1940, married 6-15-1963 Beatrice Zeigler, born 9-7-1941, ciaughter of George and Martha Zeigler. Robert M. Dickerson, born 8-201941, married Patricia Whitledge, born 9-7-1942, daughter of Thomas and Etta L. (Butt) Whitledge. One son, Robert M. Dickerson, Jr., born 11-261962. Dorlene Ann Dickerson, born 10-11-19+3. Irah Juhnson, born 9-17-1923, married Granville E. Hastings, born 1-27-1923, son of Joshua and Clara (Warrington) Hastings. One child, Granville E. Jr., born 9-21-1951. Granville R. Johnson, born 6-13-1926, married Pauline Timmons, born 4-18-1920. One son, Granville R., Jr., born 3-251955. Elwood M. Johnson, born 8-22-1930, married Marie Flood, born 2-15-1934. Four chilcien, Marie May, born 6-9-1952; Linda A., born 9-11-1954; Iualene N., born 5-22-1959; Quintin R., born 225-1961. Betty Ann Johnson, born 11-22-1931, malried Robert Vincent West, born 3-16-1931, son of Edivard West. Two children, Eruce V. West, iorn 3-12-14.j2; Brema Lee Wust, born 9-1319.8. Harold $T$. Johnsom, born 11-4-1.142 The above information received from Mrs. Lula Johnsun.

Hattie E. Johinson, born 4-23-1897, married Charles W. Short, born 5-14-1893, died 12-201957. Buried St. John's Cemetery. Son of Peter M. and Sarah Short. Anma Marie Short, born 6-919:9, died 1-15-1920́. Buried at St. John's. Hazel Short, born 11-30-1920, married Leslie E. Timmons, son of Thomas Timmons. One son Ewing Timmons, born $\bar{\jmath}-23-1940$. Charles W. Short, Jr., born 9-2-1922, married Elva Spicer, daughter of lawrence and Pearl (Fleetwood) Spicer. Charlene Short, born 1943, married Roger Calhoun, son of Ward Calhoun. Kenneth Short, Jorn 1949. The 

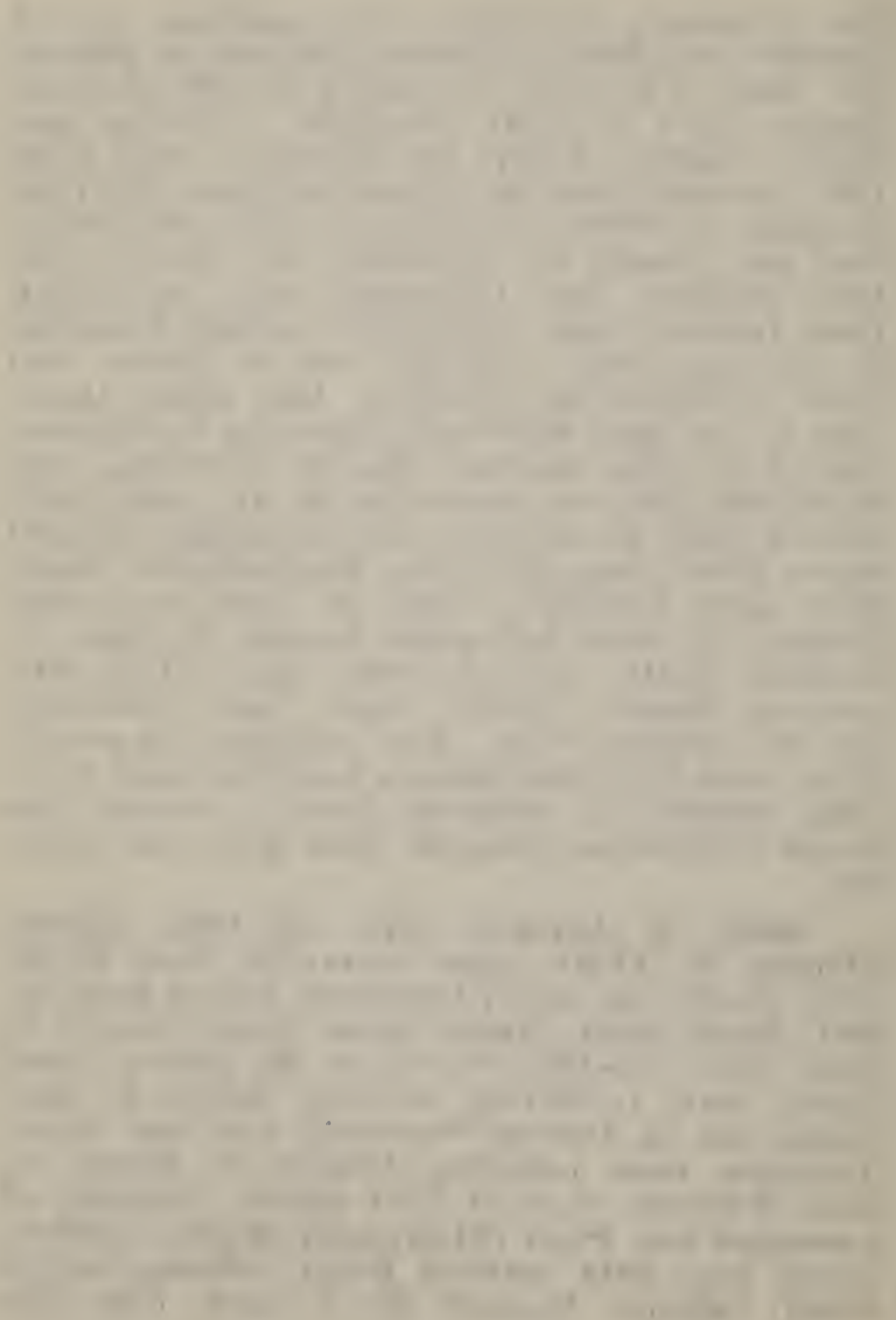

(2) 
apove intormation recelved trom ivr. Hattie short of Millsboro.

Mellie Johnson married Ben Emory. One daughter Nellie Emory married Reynolds French. One son, Reynolds French, Jr.

Harry Johnson, born 1903, died 19/8, buried St. John's Chumeh Cemetery. Any Jolinson dien in infancy.

Hezekiah Joseph, brother-i, ' v to Wm. Joseph, born 11-2-1820, died 3-20-1891, married Jane - born ..._..., died 1-23-1879. Both buried in the Megee private cemetely near Zoar. Erusmus. M. Joseph, born 1850, died 1929, married Elizabeth born 1844, died 1927. Both buried St. George's E. Chapel Cemetery. The following data received from Mrs. Amanda Carmean. Annie Joseph, born 5-4-1356, died 4-13-1925, married Tilghaman Robinson, boru 1-7-1847, died 14-1920. Both buried Conley's Chapel Cemetery. Son of Parker and Mary Ann (Simpler) Robinson. Rodncy Robinson, born 1875, died 1933, married Atdie E. Hazzard, born 1878, died 1960. Both buried Lewes Methodist Cemetery, Lewes, Del. One son, Paynter Robinson, born 1901, died 1917. Buried same place. Alfred I. Robinson, born 1878, died 1935, married Emma R. Lawson, born 1870, died 1948. Both are buried at Conley's Chapel Cemetery. Luther $W$. Robinson, died 2-23-1883, aged 2 months. Hattie Pobinson, born 1884, died 19.12, married Ferdinand Paynter, born 1879, died 1943. Both buried Lewes MTethodist Cemetery, Lewes, Del. One daughter, Edna Paynter, born 7-24-1904, married Earl Rust, born 6-4-1898. Son of G. Fred and Arlie (Cooper) Rust. Two sons, Earl Ferdinand Rust, married Louise Penuel. Two sons, Ronald E. and Donald E. Donald G. Rust married Sandra Pepper. One daughter, Penny l.cigh. 


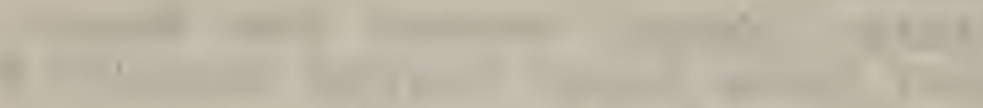

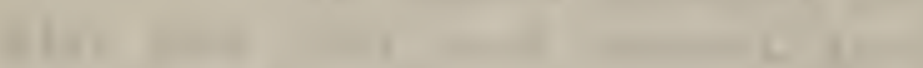

1

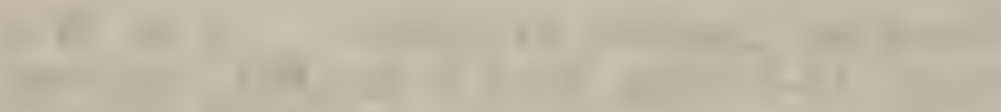

and

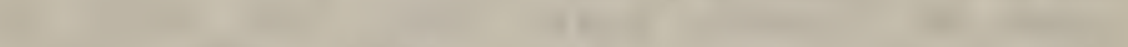

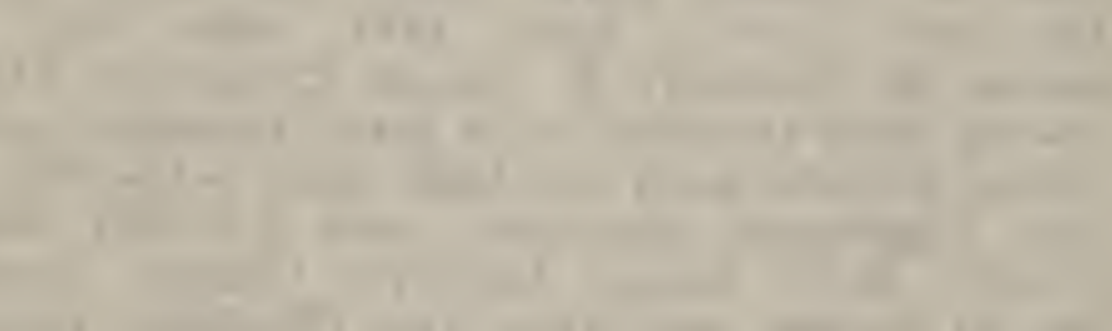

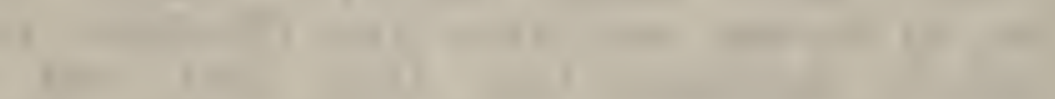

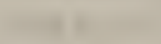

10

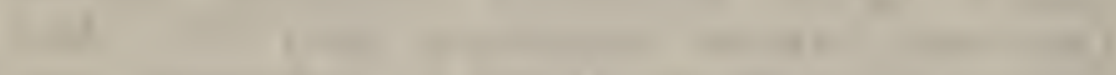

1200

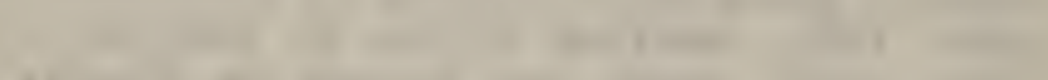

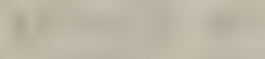

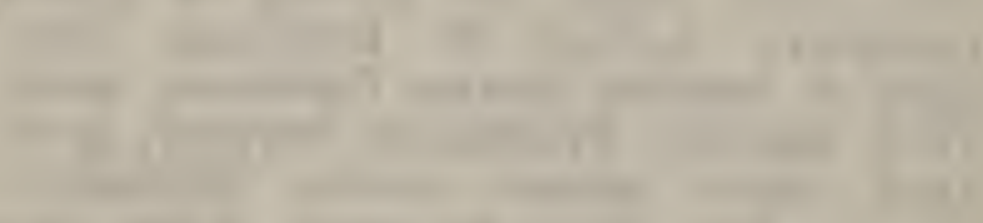

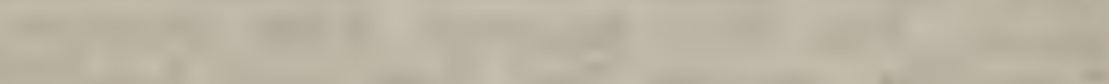

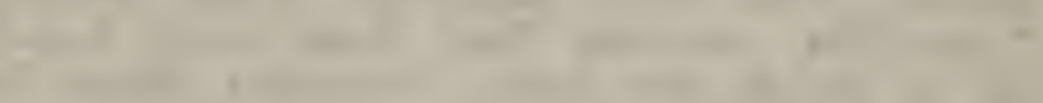

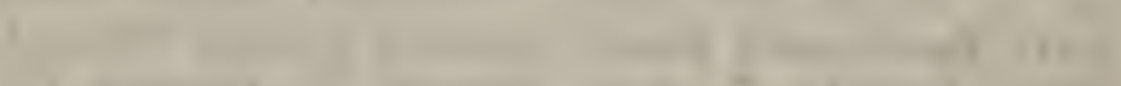

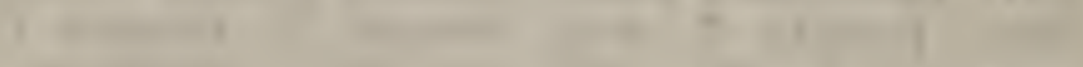

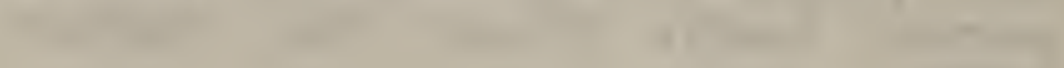


The following information received from Mrs. Amanda Carmean. Franklin P. Joseph, born 914-1353, died - married 11-27-1879, Sarah L. Steele, born 9-6-1859. Both are buried in Beaver Dam Cemetery, Harbeson. Daughter of Thomas and Frances Steele. Anna M. Joseph, born 7-271880, married 9-20-1901, Peter W. Warrington. Both buried Harbeson Church Cemetery. Son of Peter R. and Mary E. (Rust) Warrington. Amanda P. Joseph, born 1-29-189t, married 9-8-1917, David Carmean. F. Donaldson Carmean, married 12-171960, Kathyn Hitchens. Three children, Carlton Carmean, married Ramona Jester. Four children, Richard, Robert, Kathy, Otis, Shelly. Alvin D. Carmean married 6-15-1946 Godys Clifton. Catherine Carmean, born 7-31-1923, died 3-17-19+3, buried Odd Fellows Cemetery, Milton, Del, mar. ried 9-12-1942, Harry W. Thomas, born 3-30-1917. No issue. Iayton U. Marmean, died 3-28-1928, buried Mechanics Cenctery, Millsboro.

Alvin D. Joseph, born 4-7-1896, died 12-31959, buried Dehnar, Del., married 3-20-1926, Sarah R. Truitt, born 1890. Iris Lee Joseph, born 8-201917, married Henry Phillips, born-2-7-1917. Son of Lawrence and Comile (Long) Phillips. One son, Robert Wayne Phillips, born 6-12-1943. The above received from Mrs. Iris Phillips of Millsboro.

Dewitt Joseph, born 1-19-1361, died 1-1-1943, married Hester Steel, died 8-22-1901. Daughter of Thomas and Frances Steel. Both buried Delmar, Jel. John E. Joseph, born 6-8-1886, married Ethel Megee, born 11-13-1805, died 8-20-1961. Buried Rehoboth Methodist Cemetery. Kenneth Joseph. Thelma Joseph. Emma Jane Josenh, born 6-28-1893, died 1-26-1963, married 9-21-1916, John eight children. Both are buried at White Chapel Mitchell, born 1895, died 2-12-1960. They had Cemetery. Hazel Mitchell married Charles Carey. 


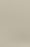

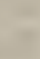

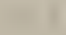

$$
\text { (1) }
$$

(1)
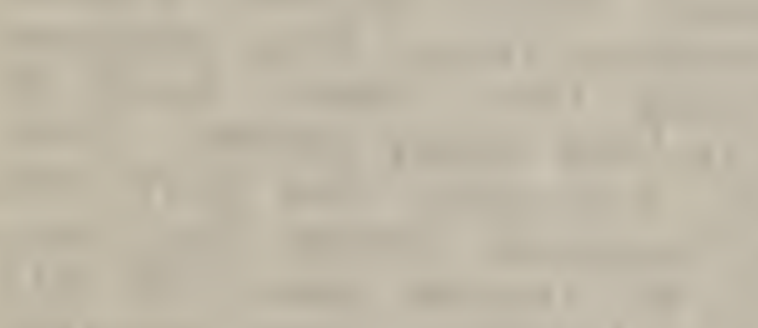

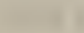
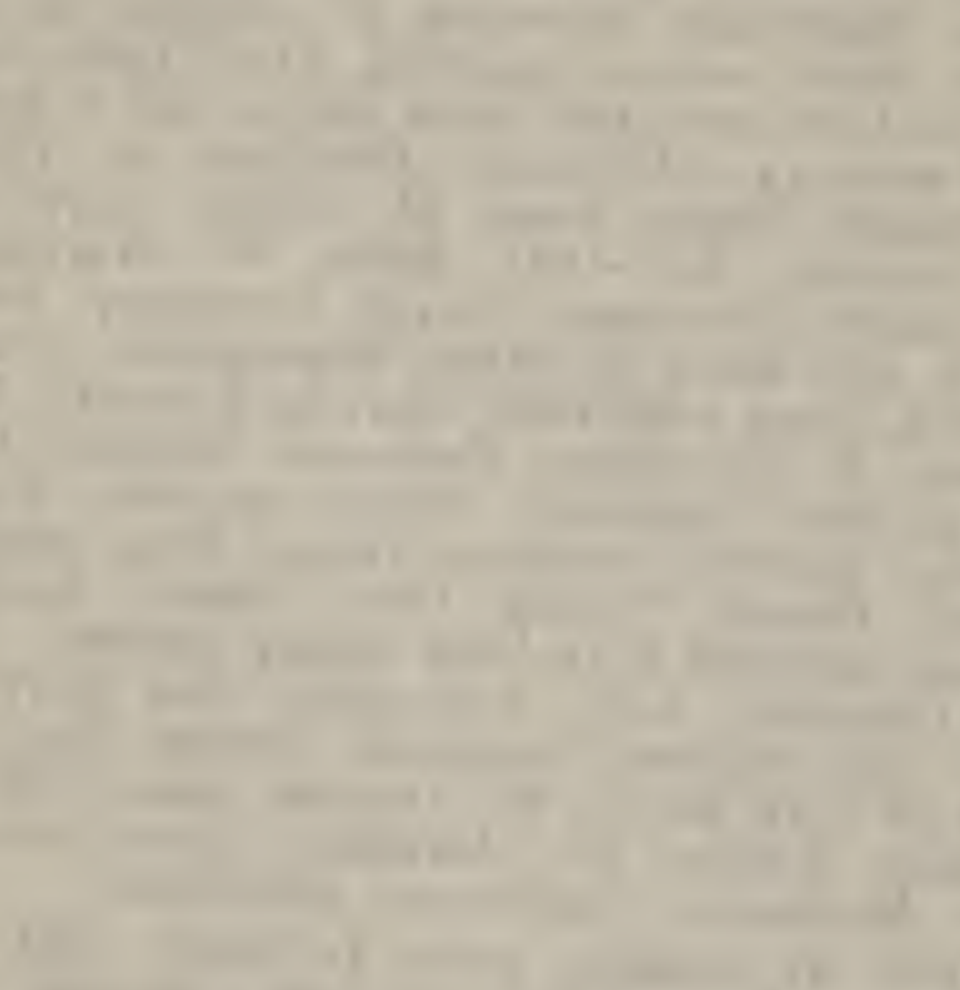

(

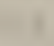

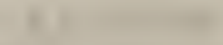

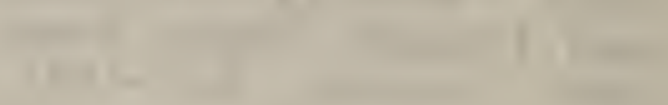

1

C

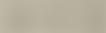

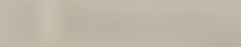

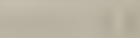

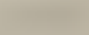

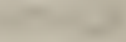

(1)

-

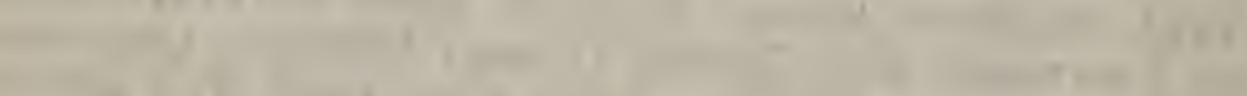

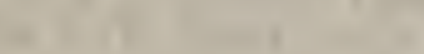

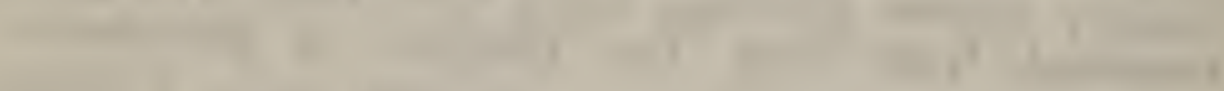

11

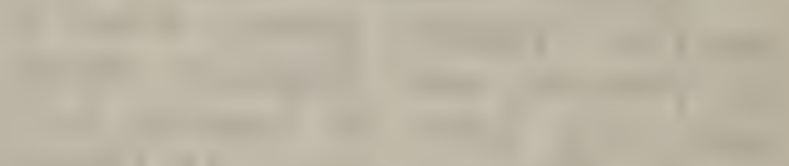

10

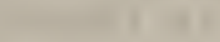

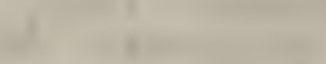

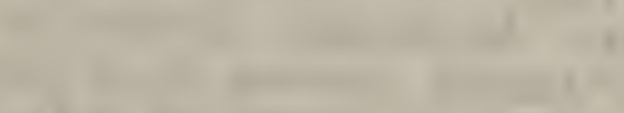

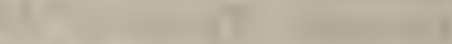

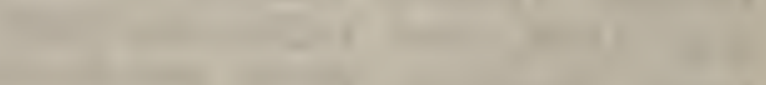

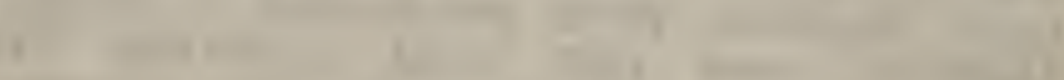

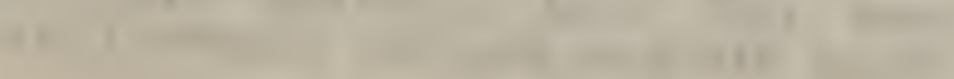


Two sons, Ronald Carey married Ann Williams. Two children, Robert Carey married Joan Slsiel. Flizabeth Mitchell married Nelson Coverdale. 'Three children Susanne, Rebecca, Dennis Coverdale married Pat Short. Doris Mitchell married Arthur Wyner. Two children David. and 'Theo. Bernice Mitchell married James Rodger's. Five children, Joanne, James, Jr., Gerald, Stephen, Peggy Lee Rodgers. Gladys Mitchell married Harold Austin. Two children Gary and Debora. Preston Mitchell married Edna West. T'vo children, Barbara Jean and Daniel Mitehell. Lester Mitchell married Peggy Butler. Two children, Mike and Pamela. John Mitchell, Ji., unmarried. The abovo received from Mrs. Hazel Carey of Lewes, Del Ida Joseph married 12-27-1904, Leander Warrington, son of Peter R. and Mary E. (Rust) Warrington. Both buried St. John's Cemetery.

Sister to William C. Joseph, Elizabeth Joseph, born 10-22-1831, died 10-11-1898. Buried Lewes Methorlist Cemetery. Unmarried. John H. Joserh, born 10-29-1835, died 12-3-1913, married Mary A. -_- born 12-14-1832, died 1-29-1911. Both buried Lewes Methodist Cemetery. Harry C. Joseph, born 1873, died 1939 married Cora.'T. Virclen, born 1875, died 1958. Daughter of Wm. Henry and Hester Virden. Clayton Virden Joseph married Lenora King, daughter of Robert J. and Julia (Butcher) King. Gilbert Harry Joseph married Sarah F. One son, Floyd G. Joseph, born 1911, died 1916. Buried Lewes Methodist Cemetery. Addie Joseph married ___ Bradford. Two sons, Clayton and Iloward. Clemintine Joseph married Michael Rogers. One son, Harry Rogers. The above data received from Mrs. Clayton Joseph. A sister to Wm. C. Joseph. Lettie G. Joseph, born 1327, died 7-24-1922, married Cornelius Robert Wiltbank, born 2-1819: died 1884. Both buried 


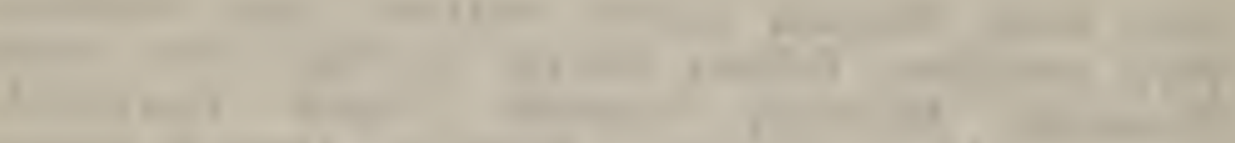

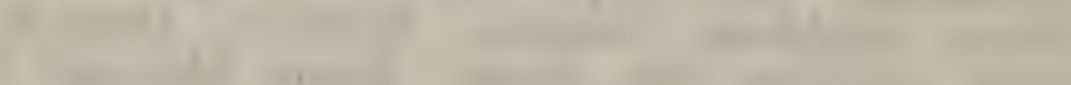
10

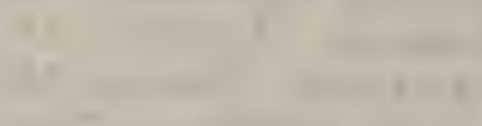

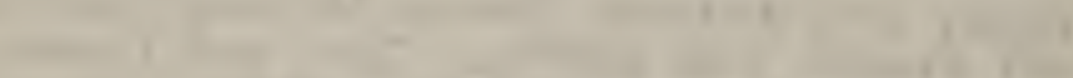

-

II

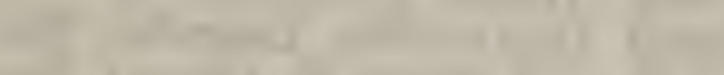

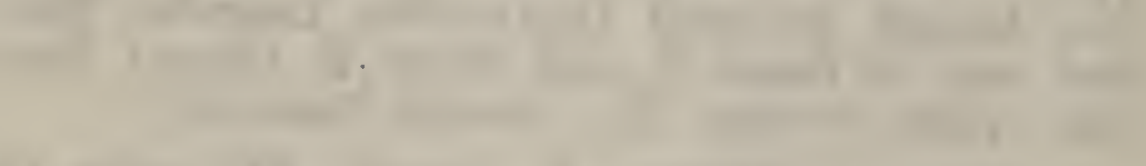

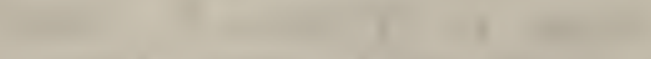

-

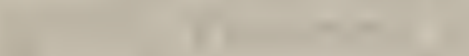

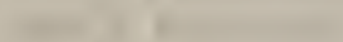

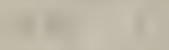

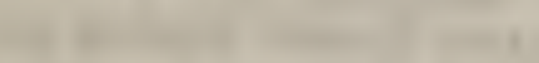

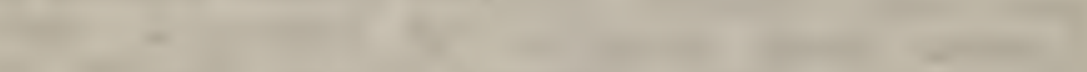

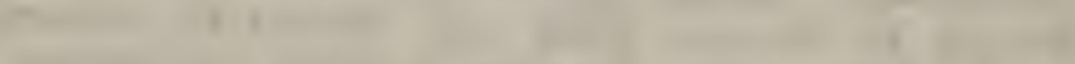

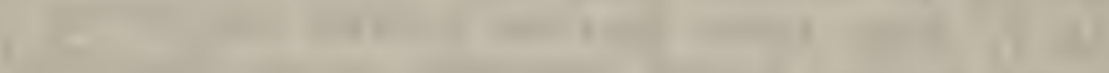

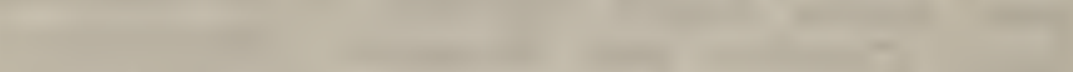

$+1$

$-4-2$

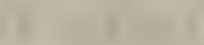

4

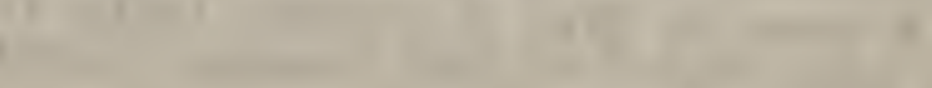

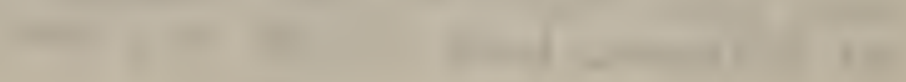


at St. Peter's Episcopal Cemetery, Lewes. Cornelius Wiltband served as Sheriff around the year 1850. Edward W. Wiltband, born 2-15-1865, died 1943, buried Lewes Presbyterian Cemetery, Lewes, Del., married Delema Russel, born 1870. Dr. Gilbert W Viltbank married Margaret ODonnell. Mr. Wiltbank is a dentist in Lewes, Del. Two childiren, Kathleen Wiltbank, born 1920; Gilbert Wiltbank, Jr., born 1919. Mildred Wiltbank married Arthur Morris. One son, George A. Morris, born 1920, died 1941. Buried Lewes Presbyterian Cemetery. Ilettie Wiltbank, born 3-8-1860, died 12-3-1950, married David Johnson, died 8-3-1936. Both are burieci in Lewes Methodist Cemetery. He was the son of Albert C. and Elizabeth (Simpler) Johnson. Albert Johnson was also the father of Robert P. Johnson who married Anna Burke Joseph. And Elizabeth Simpler was the sister to Amy Simpler who married Wm. C. Joseph. Earl Comelius Johnson. Unmarried. One Fuster son, Frank W. Johnson married Nellie Moore. One son, Joseph Johnsisn, born 10-1-1924, died 10-9-1944. Buried Ünion Cemetery, Georgetown. Elizabeth Wiltbank. The above information received from Mrs. Nellio Johnson and the Dutch House of Lewes, Del.

The following received from Mr. Harry Joseph, Mrs. Hilda Pettyjohn, Mrs. Mary Thompson and Mrs. Dot Moore. The Ninth child of Jeremiah. Nathan Joseph, born near' Georgetown, Del. Ile was a famer He married Miss Hardy. One of his sons was William Joseph, born 4-6-1786, near Georgetown, Del., married Elizabeth Salmons, daughter of Isaac Salmons. They had eleven children. One of them was Henry Dickinson Joseluh, born 1-30-1813 near Hollyville, Sussex County, married 1st., Elizabeth B. Carroll, daughter of Peter Carroll.

Dagsworthy D. Joseph, born 3-19-1839, died 


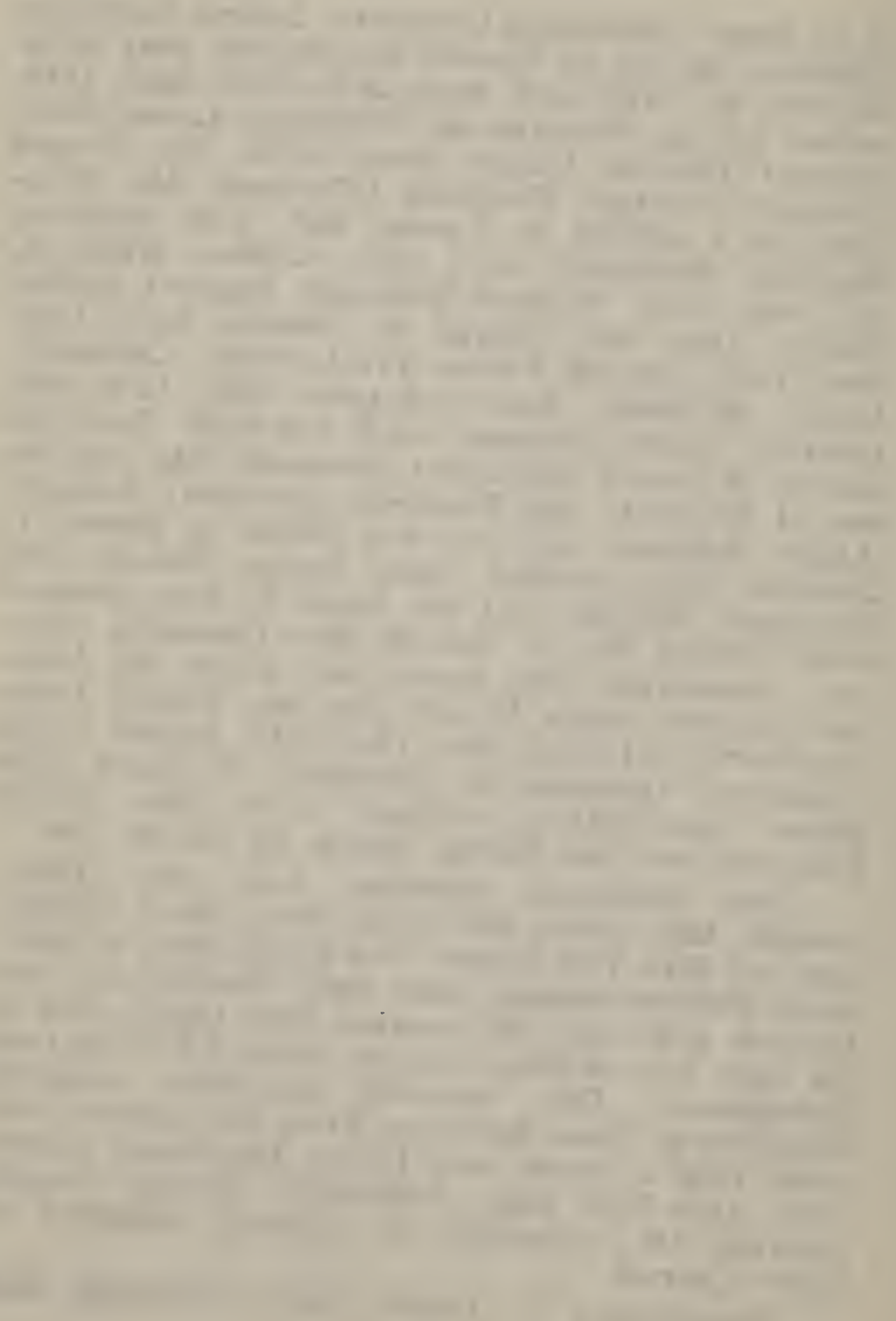


12-10-1915, buried St. John's Church Cemetery, married 6-7-1367, Mary Hester Sherman, born 3-6-1843, died 1-29-i903, daughter of Thomas and Sarah K. (Vrarrington) Sherman. Effie Joseph, born 6-19-136'7, died 10-17-1910, married Eugene Kaiser Wilson, born 1867, died 1949. Both are buried St. John's Cemetery. Son of Nath and Margaret (Veasey) Wilson. J. Carroll Wilson, born 8-11-1893, married Edith W. Cooper, born 1897, died 1929, daughter of Wilbur and Nora (Mitchell) Cooper. One daughter, Dorothy Margaret Wilson, born 10-14-1920; married J. Everett Moore, born 1-25-1921. Four children. Ronala Wilson Moore, born 12-6-1938, married Patsy McCable, born 425-1939, daughter of John McCabe, Jr. One child, Er-iє: W. Moore, born 1-6-1963. Merrill C. Moore, born 2-14-1944. J. Everett Moore, Jr., born 7-231950. Teresa Faye Moore, born 11-24-1954. Mary E. Wilson, born 1895, married Clarence Thompson, born 1884, died 1950. Buried St. John's Methodist Cemetery. Son of William R. and Irena R. Thompson. Edna Thompson married Roland King, son of Loda King. Two sons, Francis King married Louise Sammons, daughter of Lawrence Sammons. Irvin King married Nancy Abbott, daughter of John and Catherine (Shane) Abbott. Two children.

Ethel Thompson married Marshall Wilson, son of George A. and Emma (Shultz) Wilson. Richard Kenneth Wilson, orn 5-30-1937 married Joan $\mathrm{F}$. Mills, daughter of George and Grace Mills. Ronald Thompson Wilson, 3-17-1939. Joan Amn Wilson, 9-25-1940 married Roland Hitchens, son of Alvin and Ruth (Joseph) Hitchens. Robert Marshall Wilson, born 10-23-1943; James Edward Wilson, 5-2019.5; Kemneth Ray Wilson, 10-22-1946; Rodney Wayne Wilson, 1-30-1950. Harriett Thompson, 1923, married 1st., Harry Clausen, son of Harry and Charalotte Clausen. Five children, Robert Clausen, 


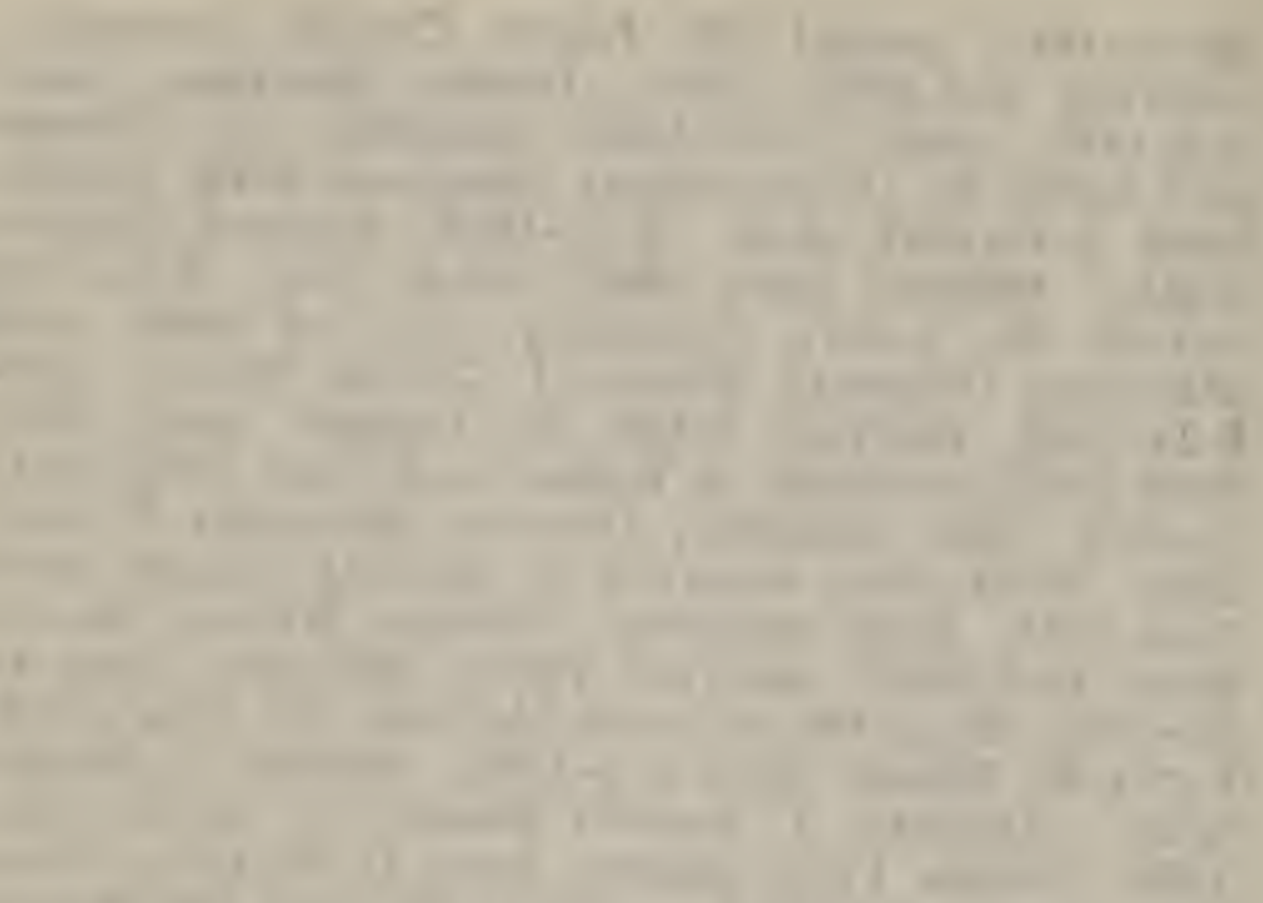

\section{$x^{2} 5^{\prime}=y^{4}=-7$}
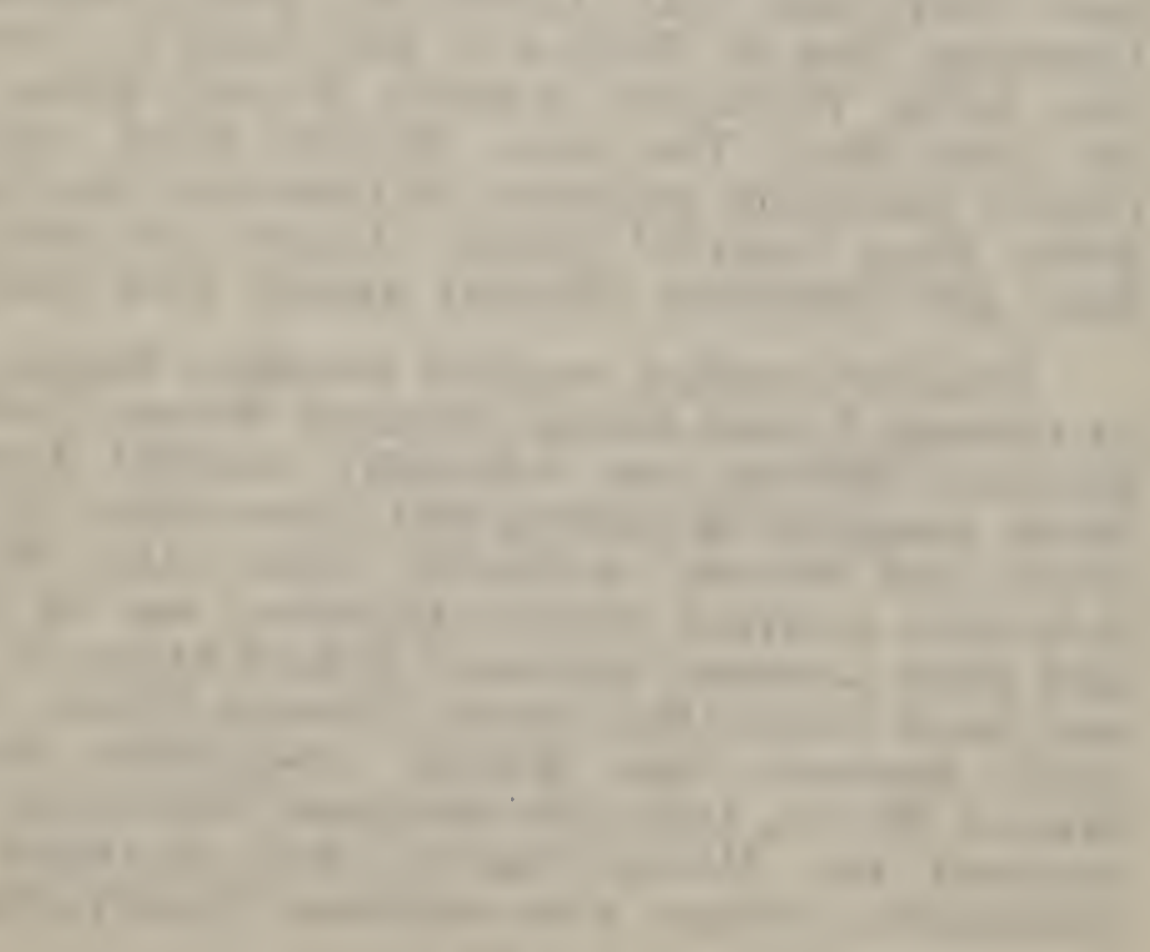
born 1943; Betty Clausen, born 1946; Ralph Clausen, born 1948; June Clausen, born 1950; Joe Ann Clausen, born 1958. Harriet married 2d., Howard Massey. One chilc by this marriage. Helen Thompson, died 1923. Helen and Harriet were lwins, born 1923. Grace M. Thompson, born 1925, died 1943. Both at St. John's Cemetery.

Clara Wilson, born 2-20-1298, died 11-10-19+2, married Wm. Ernest Wyatt, born 5-17-1893, died 10-14-1956. Both buried St. John's Cemetery. Son of William and Lizzie (Crouch) Wyatt. No issue.

Lena Wilson married Silas Downes, son of Charles Downes. Marie Downes, born 1921 amrried William Taylor. Two children, Glenm, Ann Taylor. Margaret Downs, born 1923 married Chester J. Walls, born 19--, son of Joseph and Mary Emma (Joseph) Walls. C. Preston Downs, born 1924, married Marie Hagans. Five children, Charles $P$. Jr.; Larry; Donald Arnold; Sharon; Patricia. Edith Downs, born 1927 married Francis Norton. 'Two children, Elaine; Diane. Harold C. Downs, born 1931 married I vah Brasure. Three children, Janice Fay; Shirley Ann; Stephen Allen. Shirley Downs, born 1937, marricd Melvin C. Hennessey, Jr., son of Melvin C. and Mary E. Hennessey, Sr. Two children, Mike and Kim.

Dagsworthy Wilson, buried in New Jersey, married - Gene $\mathrm{H}$. Wilson married Goldie Wilson, daughter of Charles W. and Sallie (Hitchens) Wilson. Two children, Howard and Ann Wilson.

Leander F. R. Joseph, born 10-24-1869, dicd 6-22-1914 married Vinla Pepper, born 2-6-1871, died 1-8-1910, daughter of Charles T. Pepper and Louisa Gooden. Both buried St. John's Church Cemetery. Harry A. Joseph, born 3-16-1897 married Ella Mae Calloway, born 11-22-1909, daughter of Joshua W. Calloway. Melvin I. Joseph, born 


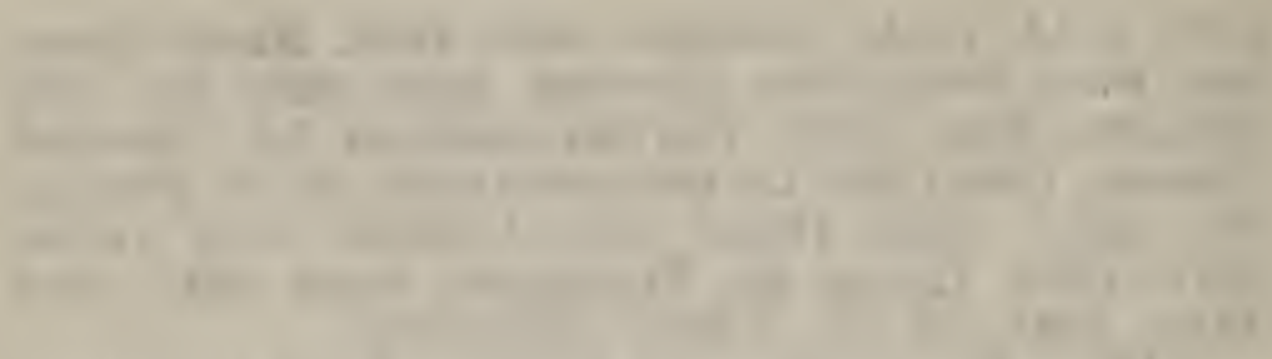

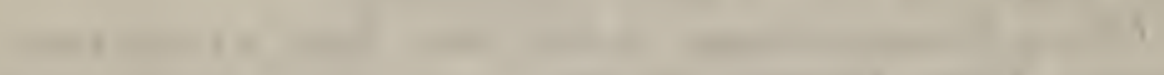

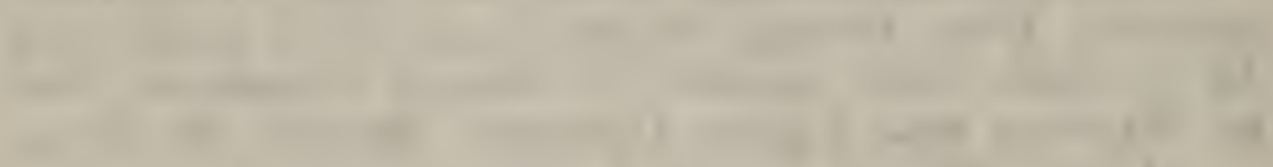

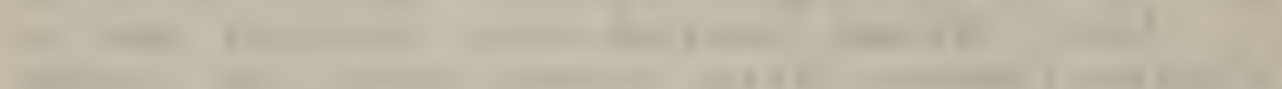

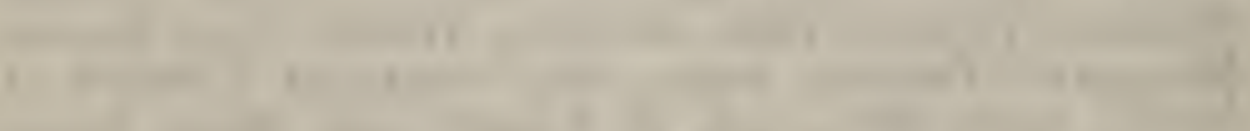
10

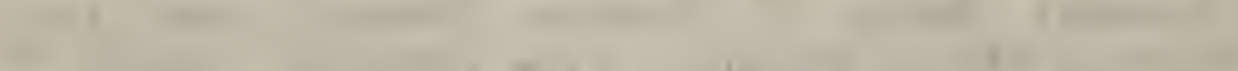

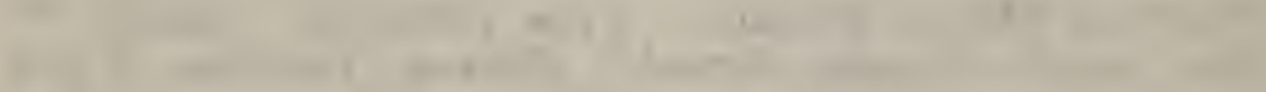

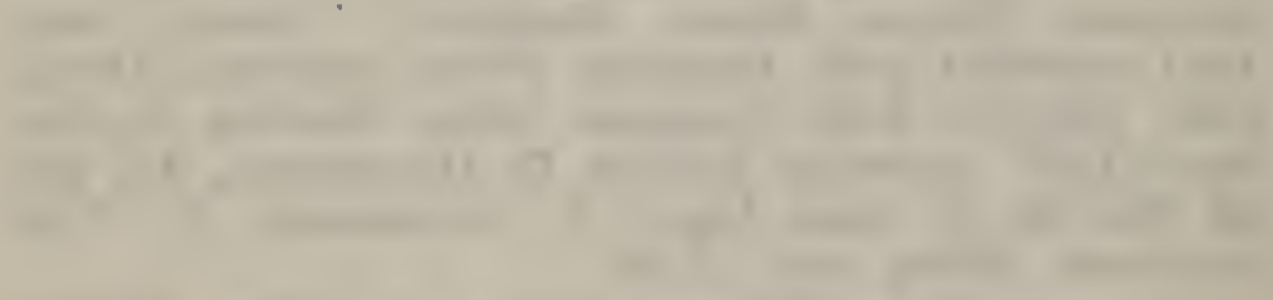

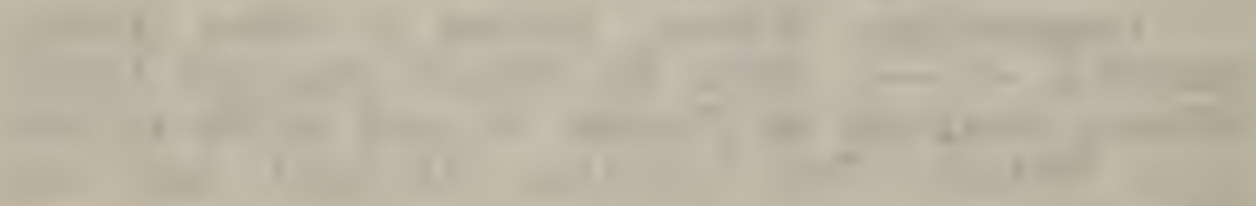

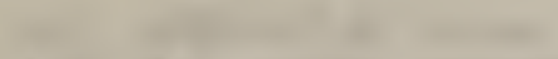

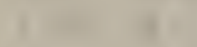

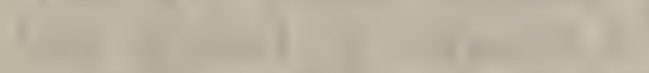

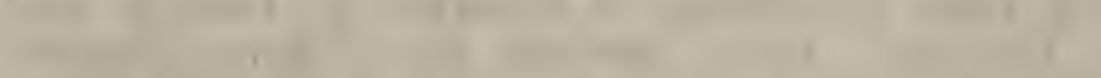

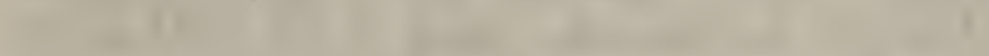

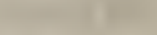
(1) 
8-4-1.921 married Pearl T'oomey, daughter of Marvel and Virgie (Wilson) Toomey. Joe Ann Joseph, born 7-24-1941 married Landreth Adams. One Gr. child, Virginia Lee Joseph. Melvin I. Joseph, Jr., born 1953. Hilda Joseph, born 2-10-- married William Pettyjohn, born 10-11-1917, son. of Howard and Julia (Veasey) Pettyjohn. Barbara Petlyjohn, born 6-17-19:39 married J. Martin Manship, Jr., born 2-10-1935, son of J. Martin Manship and wife. Two children, Randolf Keith, born 7-10-1959; Richard Martin, born 10-2t-1960. Arthur Pettyjohn, born 2-28-1941 married Carol Smith, daughter of Carlton Smith. One son, Arthur Jr. Helen Jean Pettyjohn married Charles Short, son of Fiverett and Lillie (Hayman) Short. Eddie Pettyjohn, born 1945. Thelma Joseph, born 2-6-1925 married Geo. H. Harrison. Three children, Earol I.; Geo. H. Jr.; June Marie. Loretta Joseph, born 2-11-1929 married Clifford Bunting, son of Charles Bunting. Two children, Charles Stephen; Stanley Lee. Shirley Joseph, born 10-13-19:34 married James Short. Jay Short, 1954. Janie Joseph 10-141913 married David L. Smith. One child, David $\mathrm{Jr}_{\text {., }}$ died when born. Buried St. John's. Mrs. Hilda Pettyjohn gave the above information.

Clyde Pepper Joseph, born 3-18-_ married Mildred Short, daughter of Fred and Nora (Cooper) Short. One daughter, M. Frances Joseph, born 1923 married 6-29-1947 Elmer Walls, born 3-28. 1924, son of Charles and Lulu (Coverdale) Walls. One son, Gale L. Walls, born 10-29-19:7.

Etta Joseph married Rev. W. W. Hamilton. Lcander Hamilton married Elizabeth Long. Two children, Lee H.; Wayne H. Joy I Iamilton married Ernest Hopkins, son of James and Laura Hopkins. Harold Hamilton married Libby - _- Two children, Sharon and Diane. Geraldine Hamilton 


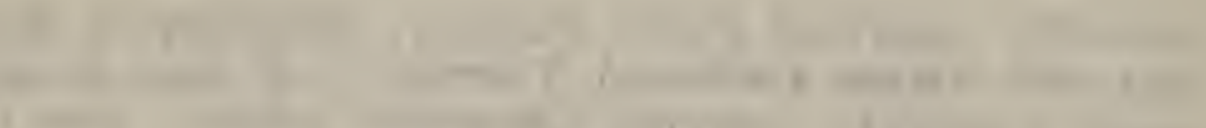

1

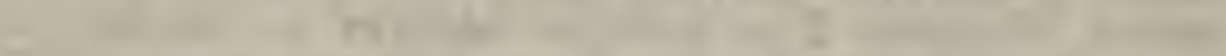

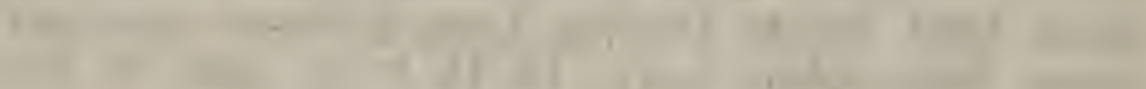

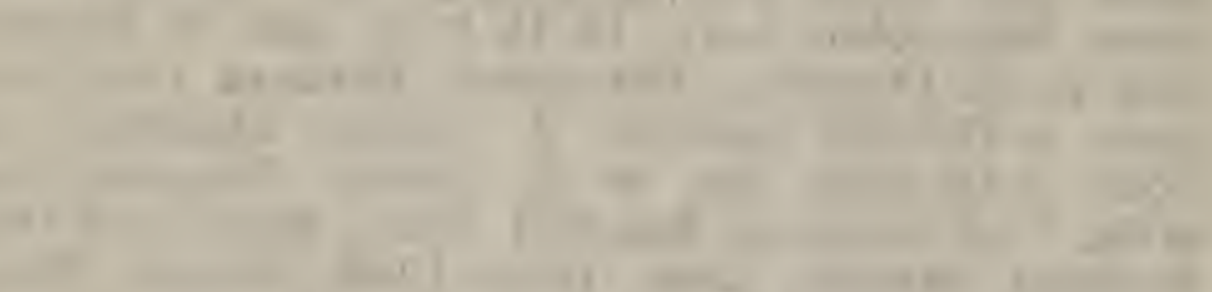

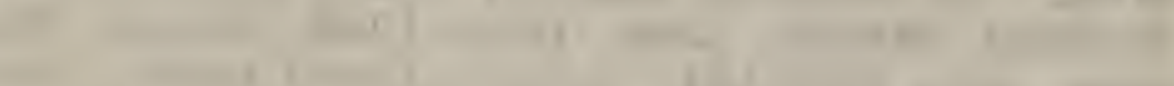

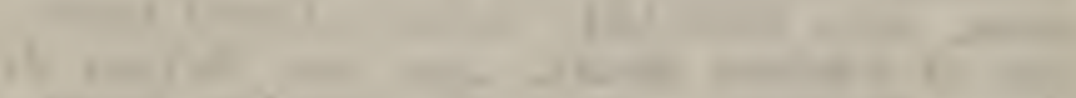

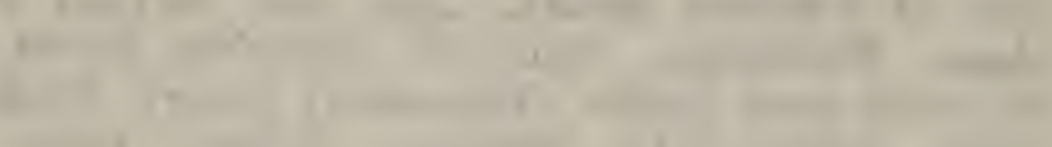

(15)

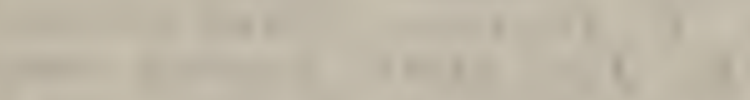

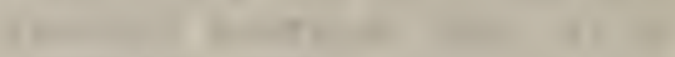

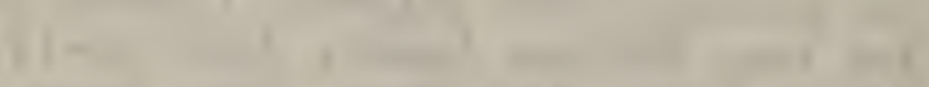

1.

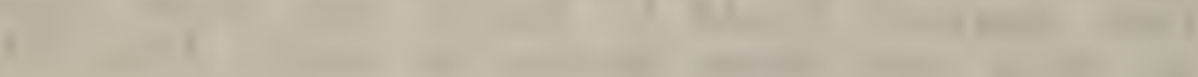

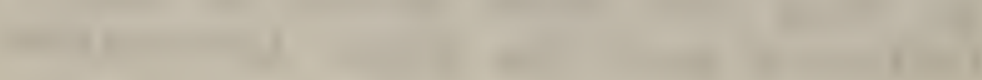

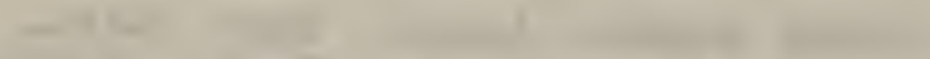

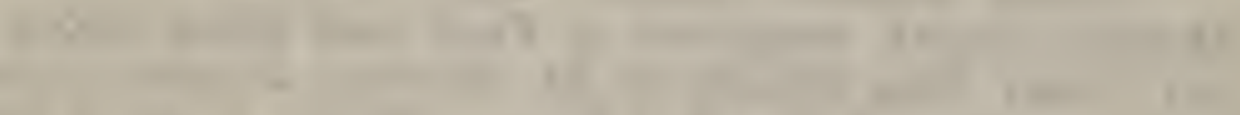

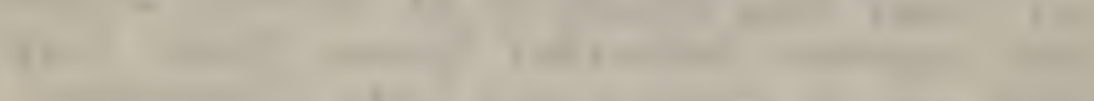

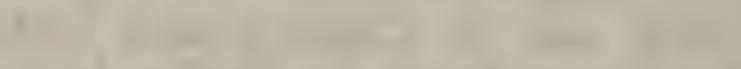

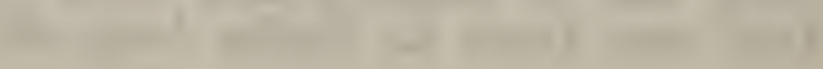

and

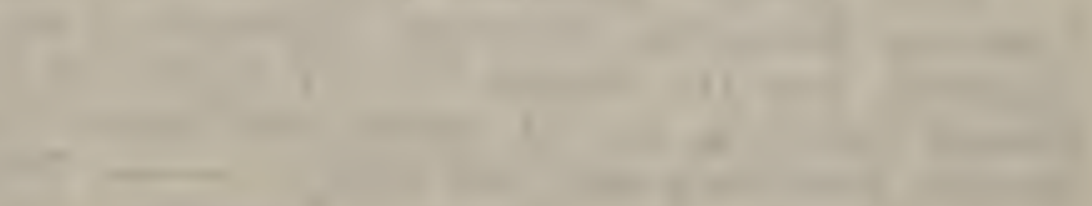

i 
married Donald Davis. They have twins, Ann and Carol.

Sarah (Sally) E. Joseph, born 1871, died 1937 married Charles Short, born 1865, died 1934. Both buried St. John's Cemetery. No issue.

Henry C. Joseph, born 1877, died 1891. Buried the same place. Laura S. Joseph, born 1877, died 1891 . Buried the same place. Nary S. Joseph, born 11-12-1830, died 1960) married Phillip R. Layton, deceased. Both buried in Bridgeville Cemetery. No issue. The following received from Mrs. Joseph Holsun. Jennie Hall Joseph, born 1884, died 1951 married Elwood Holson, born 1884, died 1942. Both buried Union Cemetery, Georgetown. Joseph Holson married Mary Sammons, daughter of James Elias and Rhoda (Junes) Sammons. Josephine Holson marlied Swain. Two children, Eric Mark Sirain; Jeanie Lynn. Barbara Halston married Frank Betts. Three children, Debra Lee; Billy Joe; Allan Wayne. Mary Beth Holson married Wilbert Adams, son of Isaac and Pheo:)e (Parker) Arlams. Three children. Yvonne Elizabeth; Marlen Lynn; Elaine. Palsey Holson married William A. Payne, Jr., son of William and Ethel Marie (Williams) Payne, Sr. One child, Venetta Marie Payne. Joseph Holson, Jr. Norman Lee Holson. Elwood Holson, Jr., married Rhoda Sammons, daughter of James Elias and Phoda (Jones) Sammons. Two children, Phillip Lee Hulson; Phylis Grace Holson. Laura Holson married 1st. Cillbert Holt. Tiva children, Connie Iolt; Giblhert Holt, Jr. She married 2d. Carey Clifton, son of Fred and Emma (Spencer) Clifton. Mary Holson, born 1908, died 1933. Buried in Union Cemetery, Georgetown.

The following received from Mrs. Maude Cullen and Mr. Ebe Rust. Maude Joseph, born 9-18-1886 married Lee W. Cullen, born 6-11-1884, ried 2-11-1958. Buried Union Cemetery. Son of 


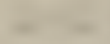

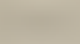

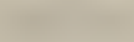

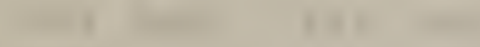

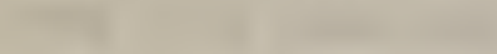

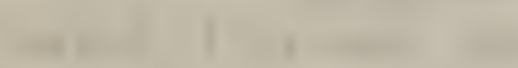

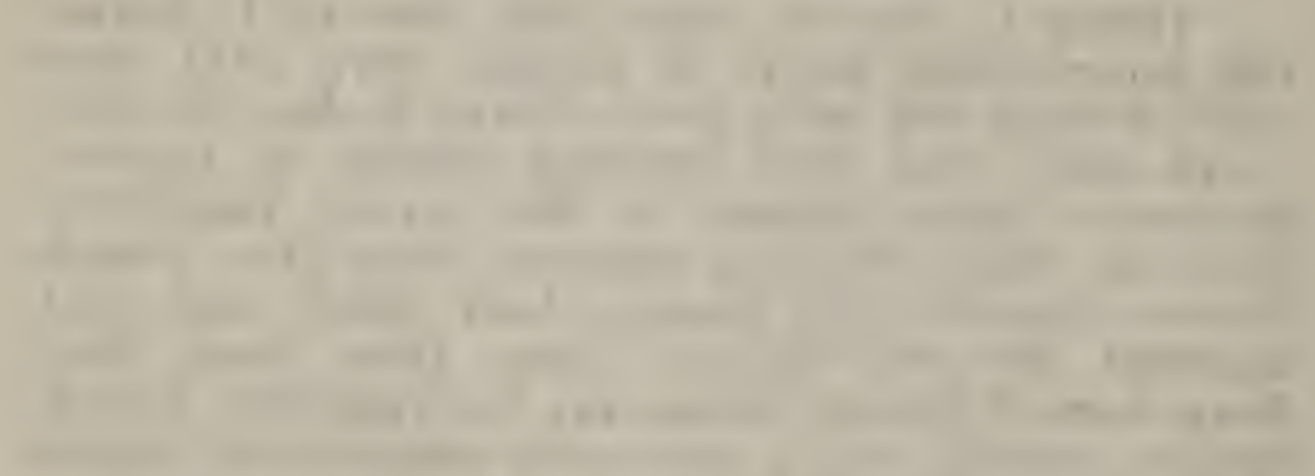

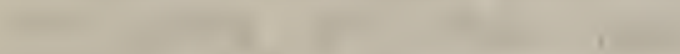

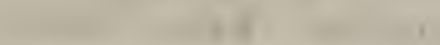
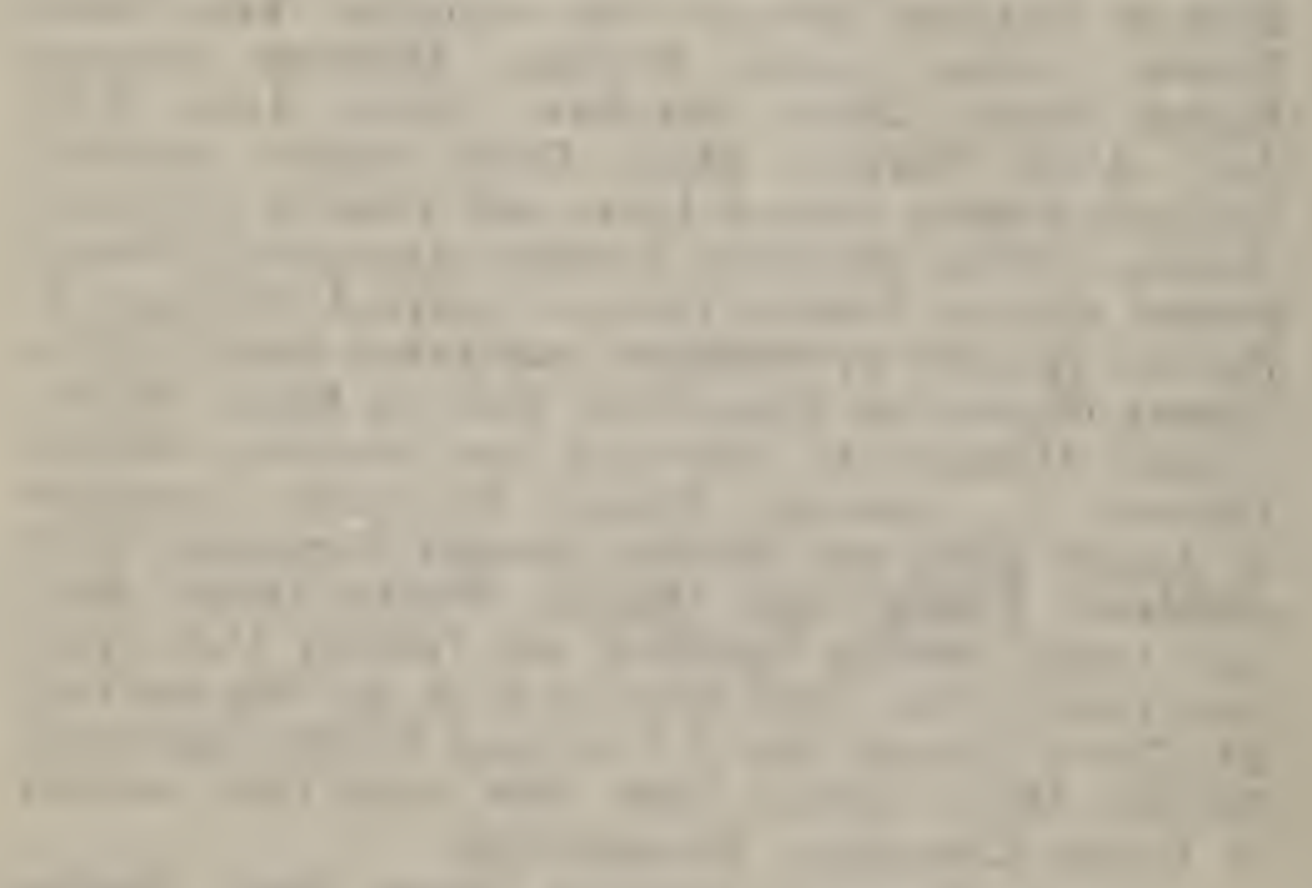

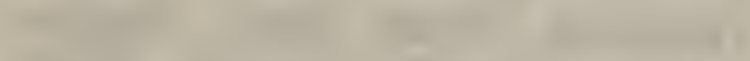

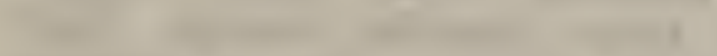

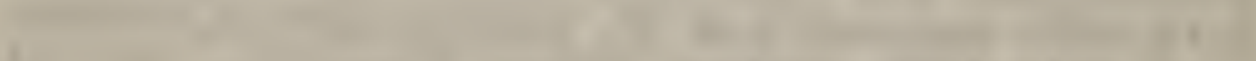
1

$\sqrt{2}$ 
Elisha Beverly and Ellen (Wheatley) Cullen. Margie Virginia Cullen married Leo Melson Bradley. One child Margie Lee Bradley. married John Allen Sims. Three sons, John Bradley Sims, bor'n 1956; Stephen W. Sims, born 1958; Keith Allen Sims, born 1960. Charles Mason Cullen married Margaret Pusey. 'Two sons, Charles M. Cullen; Richard Lee Cullen married Susan Stull. Richard Edmond Cullen married Mabel Leitch. Three children, Robert Clymore Cullen; Catherine Ann Cullen; Christopher Lee Cullen.

Other children of Henry D. and Elizabeth C.. Joseph. Ann E. Joseph married Thomas B. Rust. Both buried St. John's Methodist Church Cemetery. James H. Joseph buried in Wilmington married _-... One daughter, Laura Joseph. William Joseph died 11-14-18:62 while serving the Union in the Civil War. John W. D., died in infancy.

Family' of 'Thomas B. and Ann E. (Joseph) Rust. George Fred Rust, Sr.; born 8-24-1869, died 2-13-1943, married 1st Arlie E. Cooper, born 1012-1873, died 4-27-1914. Both buried Union. Cemetery, Georgetown. Ola L. Rust, born 11-8-1893 married Fred King, son of Peter King. Herman J. King married Julie Dodd, daughter of Fred L. and Florence (Truitt) Dodd. One daughter, Kay King. Lawrence $R$. King married Janet Hearn, claughter of Wallace and Sallie (Givens) Hearn. Earl Rust, born 6-4-1898 married Edna Paynter, born 7-24-1904, daughter of Ferdinand and Hattie (Robinson) Paynter. Farl Ferdinand Rust, born 413-1926 married Itouise Penuel. 'T'wo sons, Earl Donald, born 9-25-1952; Earl Ronald, born 9-161956. Donald G. Rust, born 2-7-1930 married Sandra Pepper, daughter of Chester Pepper 1 child Penny Leigh, born 5-18-1962. Ressie Rust, born 2-7-1905 married - Hickman.

Mr. G. F. Rust married 2d. Ella Mary Holt, 


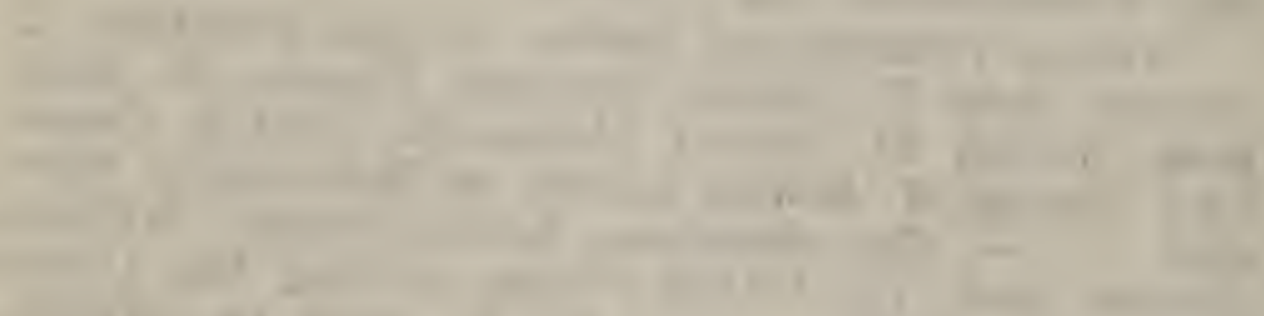

10

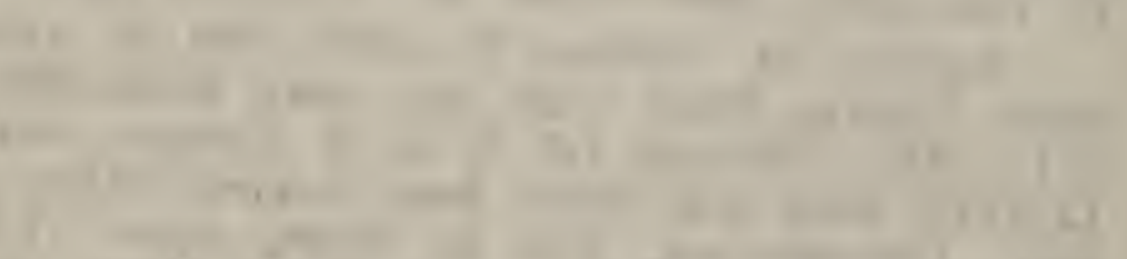

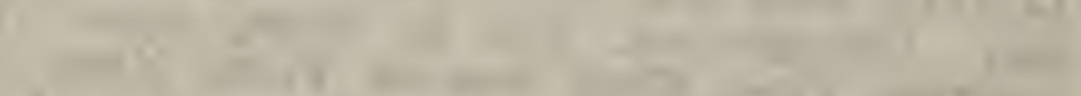

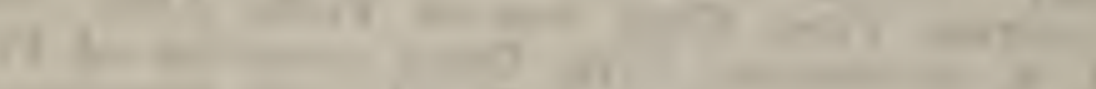
10

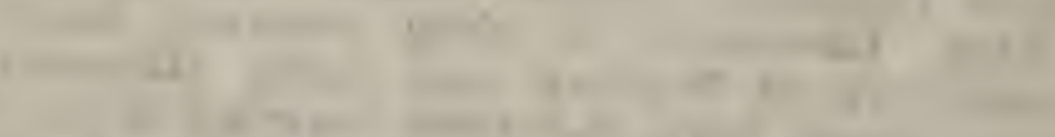

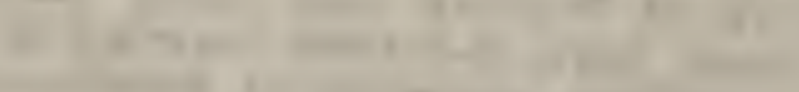
10

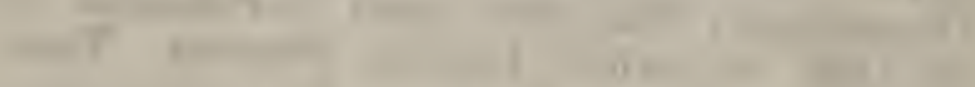
wis

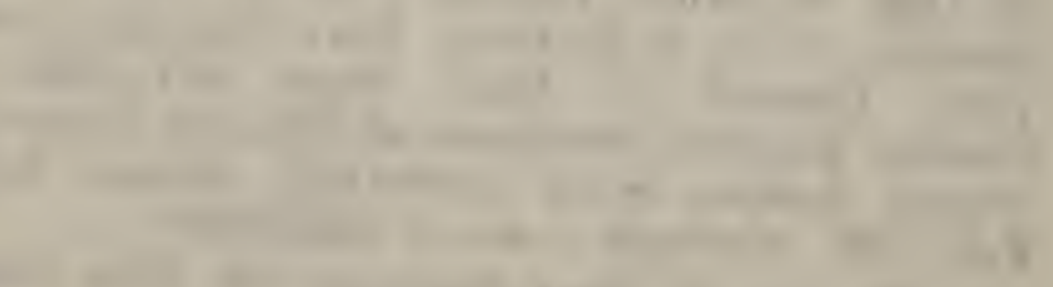

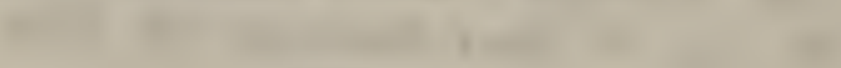


born 7-17-1891, died 7-19-1958. She is buried in Nechanics Cemetery, Millsboro. She married $2 \mathrm{~d}$. John Betts. Seven children, Raymond S. Rust' married Ruth Dykes. One chird, Nancy Rust. Louise Rust married Sebastian Simeone, son of Joseph Simeone. One daughter, Jean Simeone. Blanche Rust married Victor Epolito. Three chuldiren, Patricia, Sharon, Victor, Jr. John Rust married 1950, Eva Mae Adims, daughter of Ira Adams. One son, John, Jr. G. Fred Rust, Jr. marrier Patricia Mundorf, ciaughter of J. Grier Munciorf. Three children, Karen, born 6-17-62; Fred III, born 1-13-58; Keith, born 11-2!y-j.t. Kathyrn E. Rust, bol'n 1917, died 1961, buried St. Peter's E. Church Cemetery, Lewes, married 1st. Willarck J. Prettyman, born 1914, died 1953. Buried Union Cemetery Georgetown. She married $2 d$. Fred $N$. Willard. One son by 1st. husband, Howard Prettyman married - King. One chill. Elsanor Rust born 4-30-31 married Preston Elliott, son of John W. and Alberta (Rogers) Elliott. 22 children Christine and John.

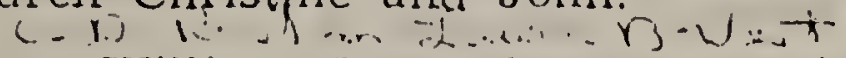

William Rust. He was married several times. We just know of his wife Nettie Brasure. Three children, John Rust; Clarence Rust. Leander Rust married Nellie West, daughter of John West. 'Two chiluren. John Thomas Rust married Sactie Cooper, daughter of George and Viola (Rogers) Cooper. No issue. S. Jeanette Rust married

( Mr. Henry Joseph married 2d. Cornelia Hill, daughter of Jehu Hill. One daughter Cornelia E. Joseph married Thomas H. Lawson of Wilmington. Mr. Josejh married 3d. Sarah Hopkins, daughter of John and Lydia (Mustard) Hopkins and. the widow of David Simpler. She died 1-10-1859, 35 years. One child b ythis marriage, George $A$. Joseph who marlied Fannie —_. One son Wil- 


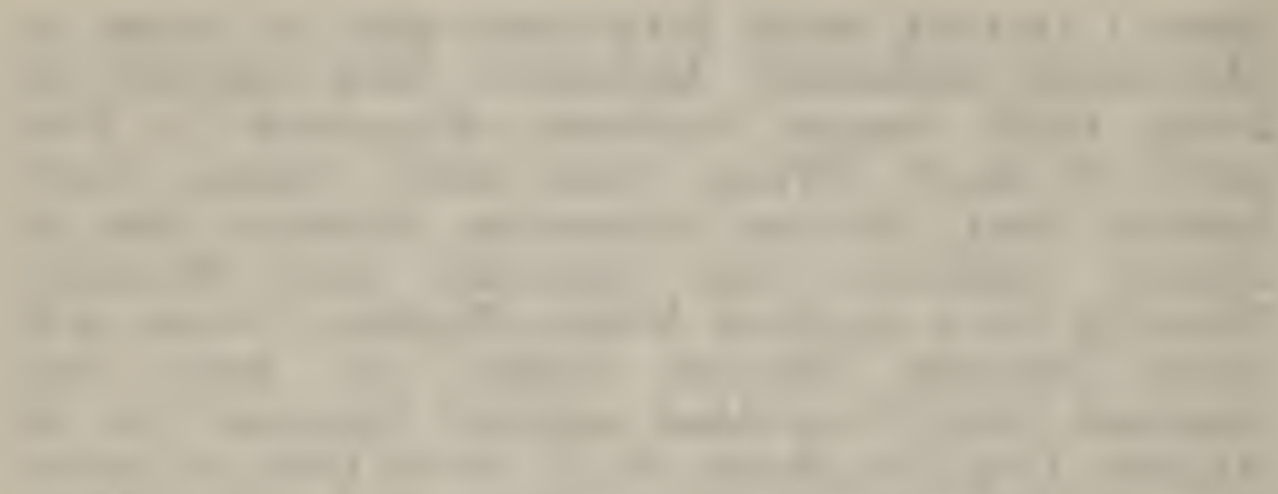

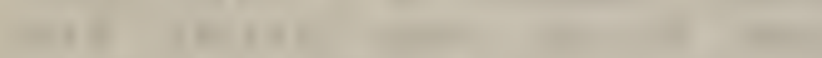
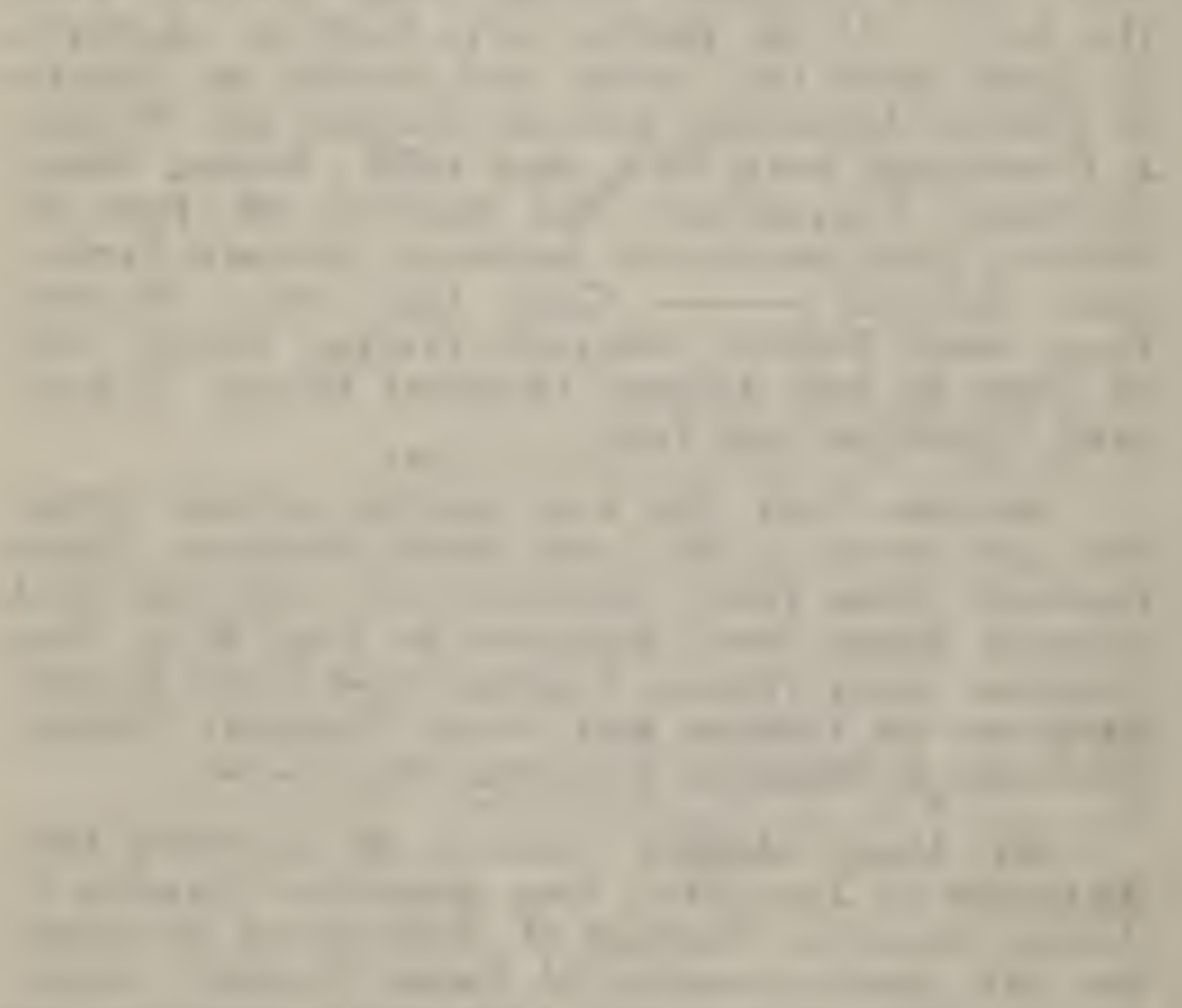

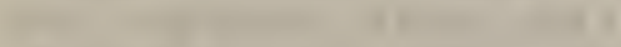

(2)

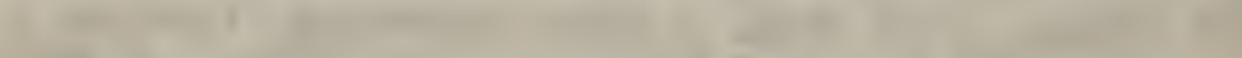

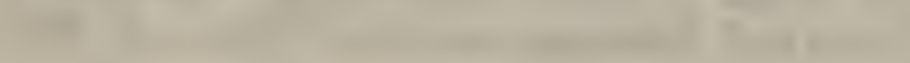


liam B. Joseph married —. One son William B. Joseph, Jr.

Mr. H. Joseph married 4th. Kate Green, daughter of John and Lydia (Rust) Green. Mr. H. Joseph married 5th. Comfort Craig, daughter of Robert and Lydia Craig. She was born 1838, died 1717. Buried Bethesda Church Cemetery, Stockley, Del. Other children of William and Elizabeth L. Joseph, Nathan H.; William B. Thomas T. Joseph, married 1838 Elizabeth Green. David Juseph. James M. Joseph. Edward Joseph. Sylvester Joseph. Gideon Joseph married Elizabeth Tindall. Sarah. Joseph. George W. Joseph married 1st. Lydia Green, born 6-9-1826, died 12-6-1847, daughter of George and Lydia B. Green. She is buried on the old Vaugh farm cemetery near Hollyville. We think that he married Katie Woodall, died, 1-15-1903, 49 years old. He had at least two children. Albert W. Joseph, born 1900, died 1962, married Leah Marshall. 'Two children, Albert C. Joseph, Jr. A daughter married Harry Grasing. Elizabeth Joseph married Cunningham. Family of Gideon and F.lizabeth Tindall. John A. Joseph born 1858, died 1905 married Mary E. Methodist Cemetery, Lewes, Del.

The Will of Jeremiah Joseph dated Feb. 14, 1824, probated Nov. 27, 1824, Will Book G-7-page 361, 1813-1827, Sussex Co. Court Honse. Quote First, I give to Eunice, my beloved wife, one-third part of my real and personal estate during her natural life, unquote. He mentions a son, Levin Joseph "All that I have advanced him, and one dollar more and no more of my estate. Mentions next, daughter Sally Joseph, son Burton Joseph, daughter Elon Magee, and son Samuel Joseph. All these to receive one dollar. 'To sor Noah Joseph, all my real, personal estate not already 


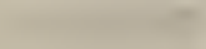

(1)
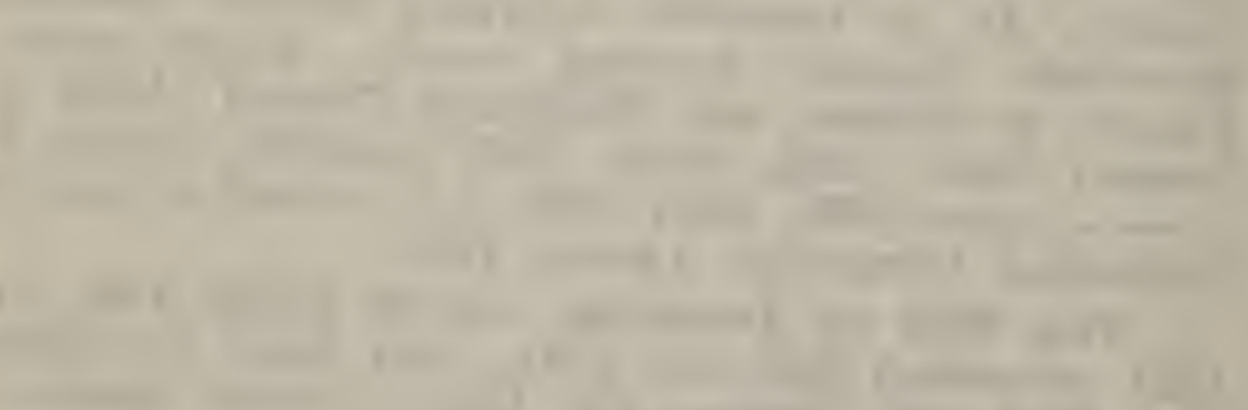

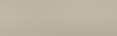
(1)

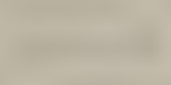

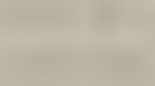

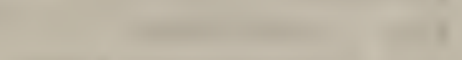

$+1$

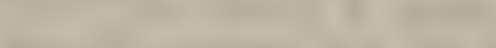

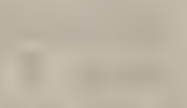

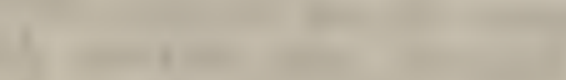

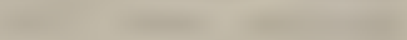

hen 
disposed of with my wife's third after her death, to him and his heirs forever. To $m y$ daughter Mary Joseph, all I advanced, and a maintenance by my son Noah Joseph, provided she will live with him as one of his family, but if she refuses to live with him, one dollar and no more .ol $m y$ estate. His wife Eunice and son Noah Joseph were named executors of his Will. Witnesses Joram Grifith, Elizabeth (Betsey) Griffith and Mary Joseph. Natlian Vickers, Reg.

W/e begin with Levin D. Joseph. Levin Donovan Joseph married Mary Ann Watson. He is buried in St. John's Church Cemetery. She is buried in Coochville, Pa. Bartimus Joseph, born 5-2-1870, died 10-27-1938 married Lucinda Bowden, born 5-(j-189t, died 2-14-1919. Both buried Odd Fellow's Cemetery. Seaford. Lottie Mae Jos$e_{1}$ li, born 1-16-1912 married Avery Isaac Tucker, Si'. Four childien Avery I. 'Tucker; 'Jr.; James Thomas Tucler; Levin Bartemus 'Tucker; Lucinda Anna lucker. Grandchildren of Lottie May Joseph Tucl:er. Avery 1. Tucker III; Brenda Ann; Cąrla Juhanna; Josephine I'a, deceased.

James Paul Joseph, born 3-25-1916 married 1st. Mildred Passwaters. Married 2d. Roberta Passwaters. One son James Paul Poseph, Jr. married Sue

Mitchell Joseph, died 2-2-1935 married Lizzie Mitchell. daughter of Joshua Mitchell. One daughter, Ollie Joseph, born 190.). Married two times. - Peter Joseph, diea 1941 married Rhoda Dayton, dier 4-.1-1949. Both buried Salisbury, Md. One son, Lawrence Joseph.

Willard Joseph died young. Buried St. John's Church Cemetery. Fred Joseph. Anna Joseph marmied James Henry Messick, his first wife. He is buried Bethesda Church Cemetery. One son Clifford, deceased, married Clara 


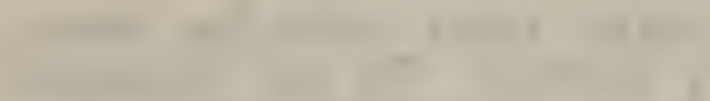

(n)

7

$+1$

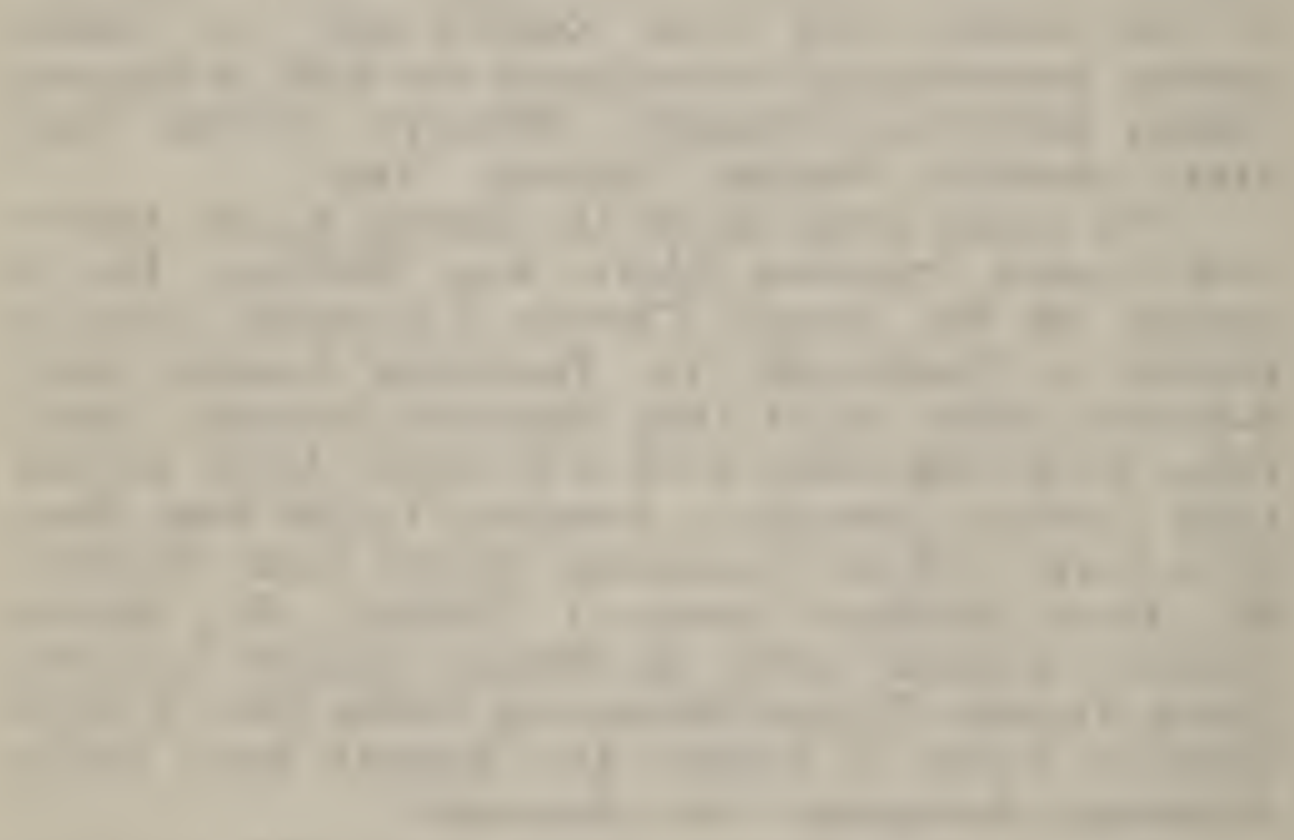

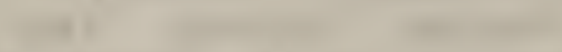

$\sqrt{3}$
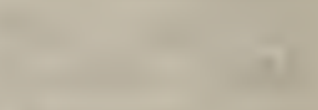

$\sqrt{2}$

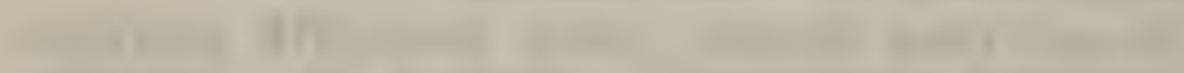

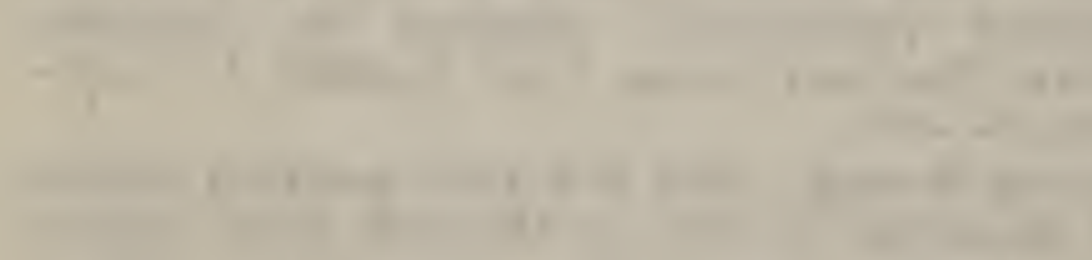

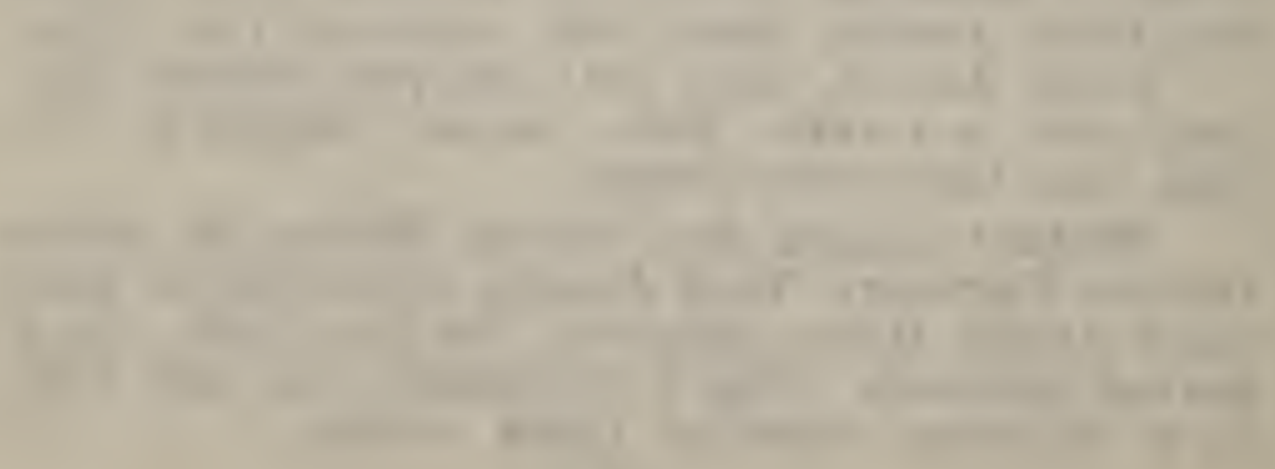


Newton M. Joseph, born 1882, died 1947, buried Mechinics Millsboro Cemetery, married Nora Long, born 9-29-1887. Son of Wm. Long. Newton M. Juseph, private, Co. First Regiment enrolled Del. Infantry Volunteers. Eniolled 5-18-1898 at Middletown, Del. Served two years. Honorably discharged from U. S. Service 11-16-1900.

Mary Joseph Drummond married 7-24-1924 Douglas A. Drummond. Two sons William D. Drunimond, born 2-25-1930 married Gilda Calvin, dazughter of Daniel M. Calvin of Florida. Theodore Drummond married Betty Jane Ward. Two childiren Shelley Ann and Trease Lynn.

Anna Joseph, born 1904 married Walter Hudson, born 1896, died 1944, son of Joseph B. Hudson. T.wo sons, Walter Hudson, Jr. ,born 2-15-1924, died 7-28-1944; Russell J. Hudson, born 1926, died 1931. Both buried Carey's Church Cemetery near Millsboro.

William H. Joseph married Betty Cleaner. Six children, Elcanor Joseph; Dorothy Joseph; George Joseph; Lenora Joseph; Ronald Joseph. Grandchildren of Wm. H. and Betty (Cleaner) Joseph, Connie Lou Josepin; Wanda Jean Joseph; Mildred Ann Joseph; Lou. Ann Quillen; Cindy Ren Quillen; Charlene Joseph; George Allan Joseph; Denise Yager; Patricia Ann Yager.

Mildred Camille Joseph, born 6-4-1915 married Phillip S. Ellwanger, born 6-7-1904, divorced. Three sons, Robert S. Ellwanger, born 10-27-1932 married Frances French, born 10-15-1928, died 3-8-1963. Two sons, Keith French Ellwanger, born 2-18-1456; Steven Martin Ellwanger, born 7-181959. Virgil Phillip Ellwanger, born 7-23-1943. Donald Martin Ellwanger, born 5-3-1948. Robert S. Elkwanger married 2d. Bernice Green. She had one son by former marriage, Roger Lewes Ber- 


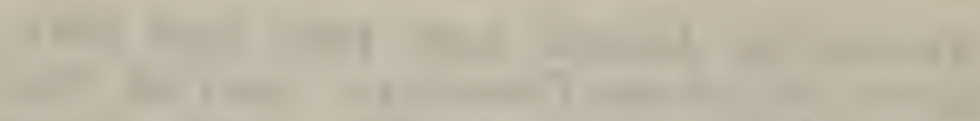

10

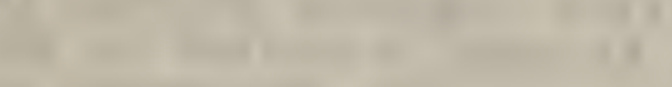

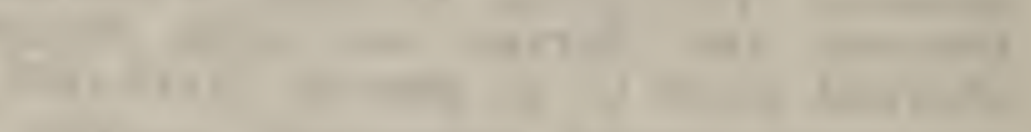

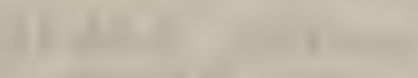

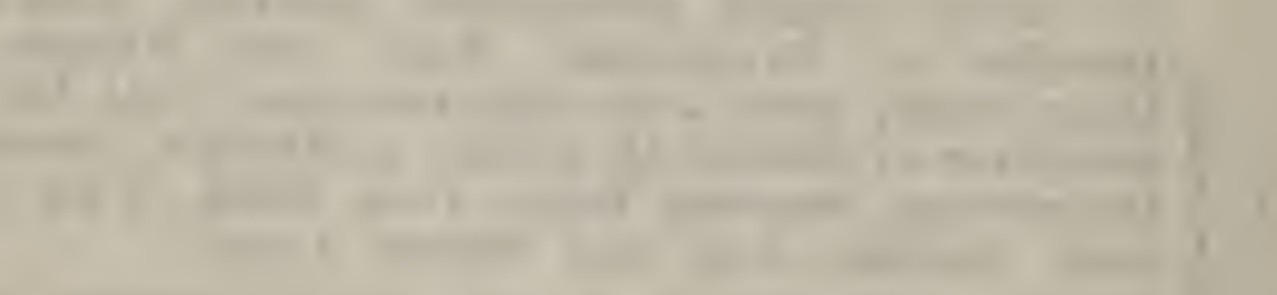

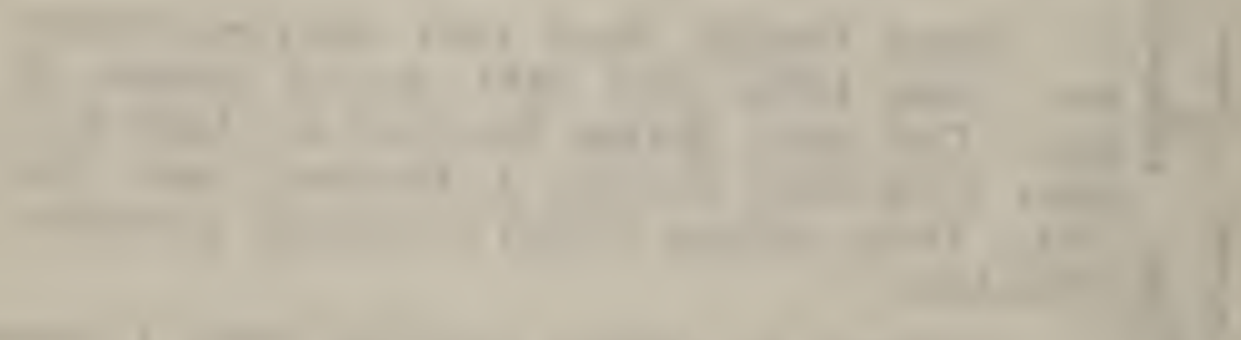

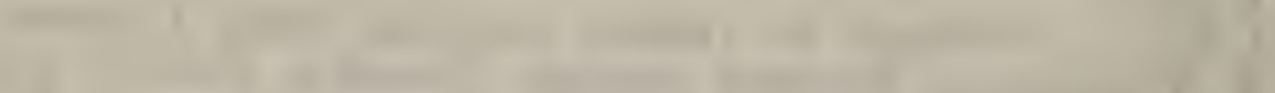

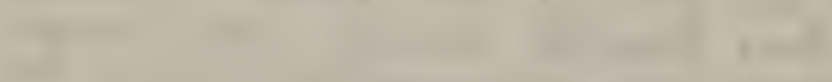

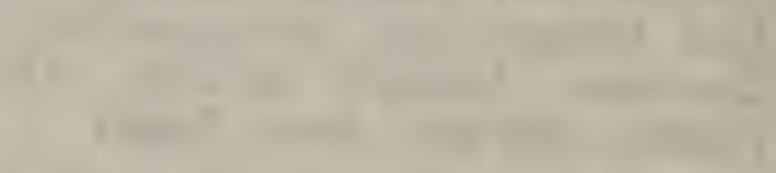

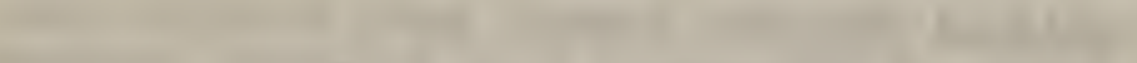

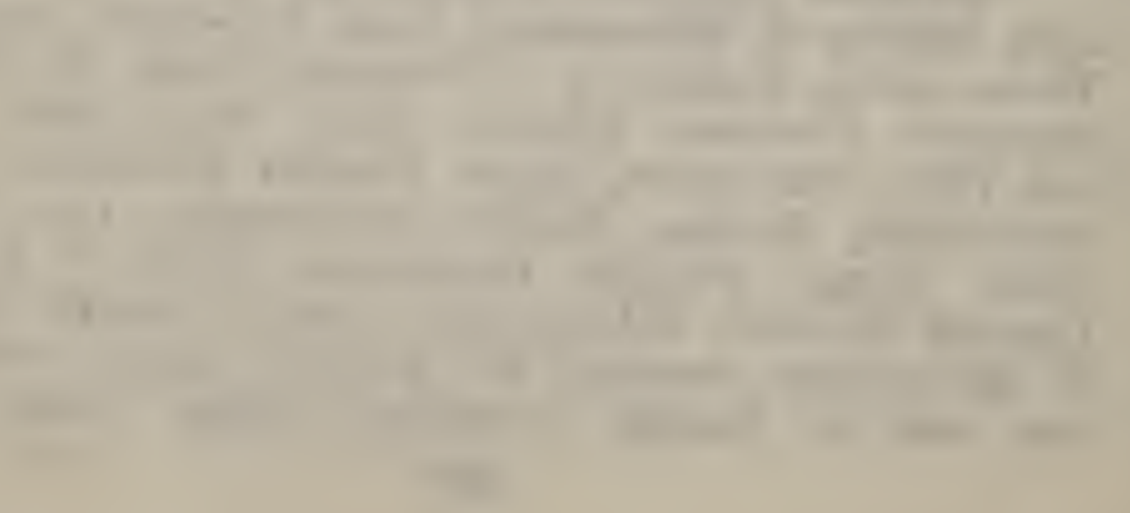


wick. They were married 4-24-1963 at San Angelo, Texas.

Sallie Joseph, born 5-6-1884 married 1st. James: Hc Kay, deceased. Four children. Henry Mc Kay married Anna or Ann Clay'. William MC Kay married Viola —. Paul Mc Kay married Noami - Myrtle Mc Kay married Gus Byring. Sallie Joseph married 2d. Charlie Eckhart. The abole information has been received from Mrs. Nora Joseph; Mrs. Mary Drummond; Mrs. Lottei Tucker; Mrs. Mildred Ellwanger; Mrs. Betty Joseph. Edward Joseph, 1924-1931.

Son of Jeremiah Joseph. Samuel Joseph, died 1797, married Elizabeth -, died 2-10-1826, 39 years. Samuel White Joseph, born 1818, died 1890, married Sarah V. Lawson, born 1824, died 1887. Both buried Ebenezer Church Cemetery near Lewes. Daughtel of Selby and Hetty Lawson. Rufus F. Juseph, 1846, died in childhood. Daniel F. Joseph, born 10-15-13+5, died 1903, married Nary Elizabeth Taylor, born 5-12-1846, died 1916. Both buried Union Cemetery, Georgetown.

Minnie Joseph married William Barbour. Na issue. Lillie Joseph, deceased, married - I'ickery, decease?. Both buried in Macon, Mo. Na issue. Hattie B. Joseph died in childhood. Frank E. Joseph died 1877, 21 years. Effie L. Joseph died 1875. 2 years. Gordon L. Jospeh died 1875.

Rufus Joseph, died 11-4-1849, died in childhook. Roland W. Joseph, born 1852, died 1911, married Sarah E. Hart, born 1858, died 1934. Both buried Union Cemetery, Georgetown, Del. Son of Gulten Hart. Franklin Joseph, born 1-12-1836, died 11-25-1952. He married Florence E Rickards, born 1891, died 1942, daughter of Stephan E. and Odelaide Rickards. One son, Ronald Joseph married Helen Lofland, daughter of Peter $C$. and 


\section{.}

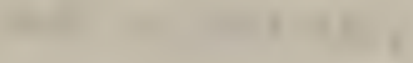

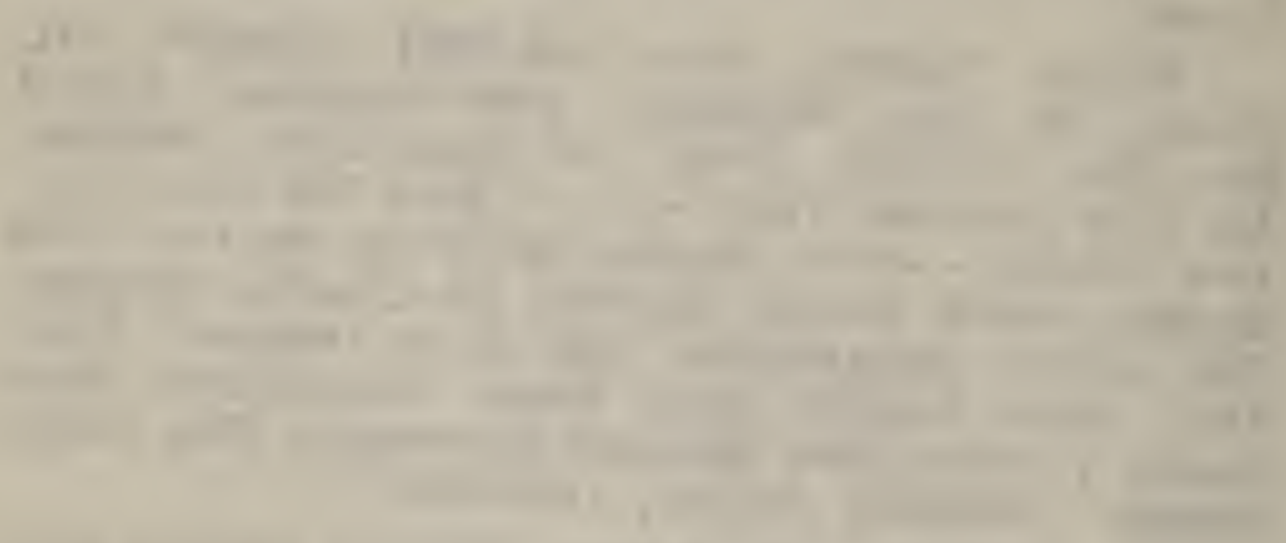

4

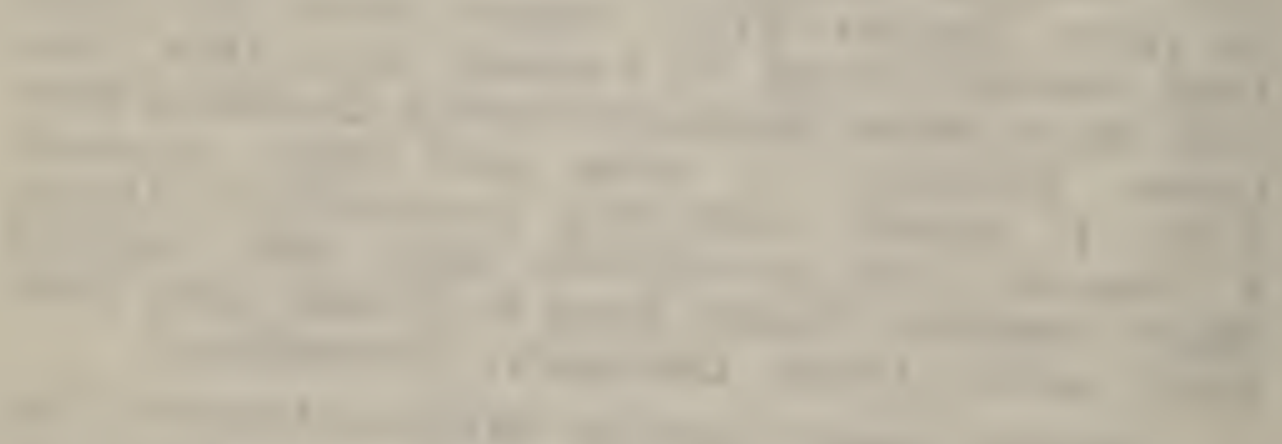

414.

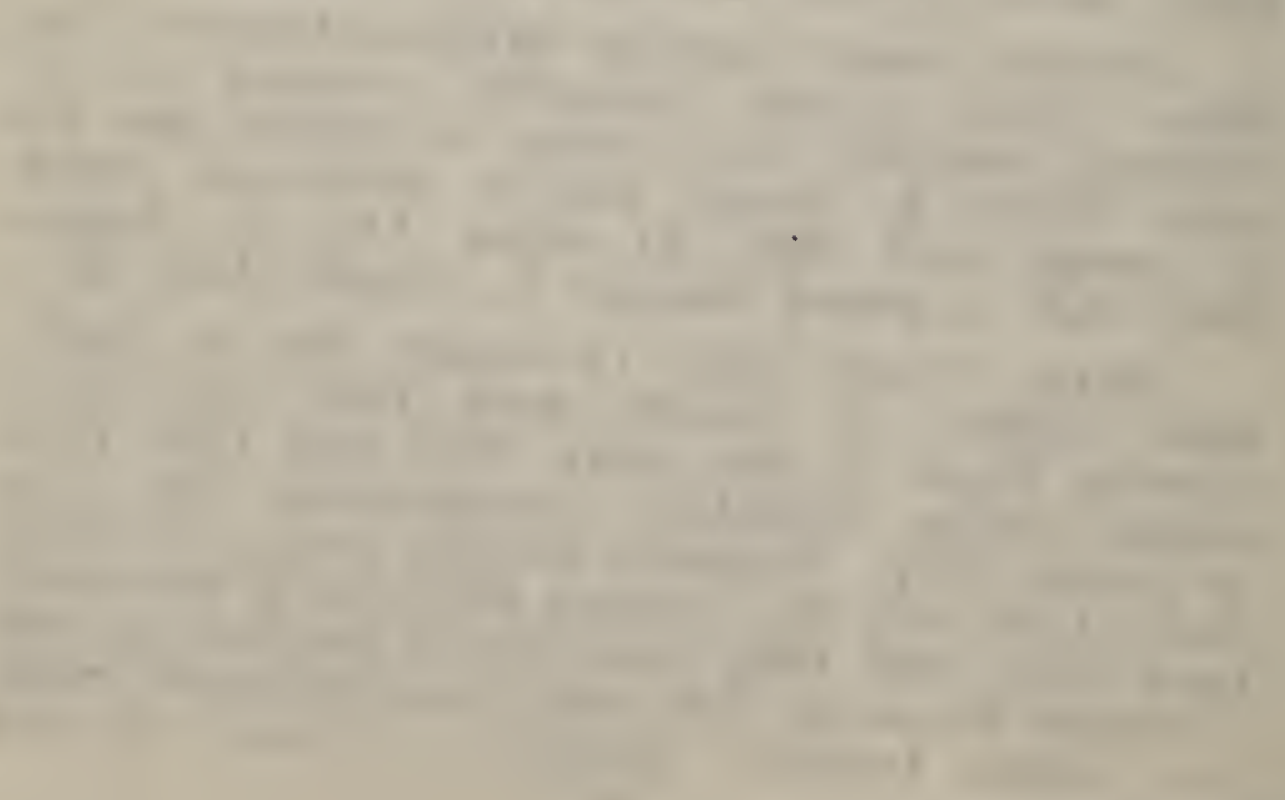




\section{0}

Elizabeth (Johnson) Lofland. Two sons, Ronald Joseph, Jr.; Paul Joseph.

Thomas W. Joseph, born 1878, died 1928 married Mary L.. , born 1879, died 1927. Both are buried in Union Cemetery, Georgetown. 'Threa sons. Loleta O. Joseph, born 5-15-1883, died 5-221959 married Gideon Walls, born 1839, died 1923. Both are buried in St. John's Church Cemetery. Son of Gideon and Patience (Rickaras) Walls, Sr. No issue. Mallery W. Joseph, born 1896, died 4-20. 1956, buried in Union Cemetery, Georgetown, married Woodford Steward, born 11-26-1897, son of Ebe and Annie (Bennum) Steward. No issue.

Letita Joseph resided in Philadelphia. Josephine Joseph, born 3-30-1848, died 1932 married Joshua Moulton Blizzard, born 1843, died 1916. Both buried Union Cemetery, Georgetown. Son of Stephan E. and Sarah (Walls) Blizzard. Ruth W. Joseph, born 9-29-1855, died 7-25-1877, buried Ebenezer Cemetery, near Lewes, married Juseph J. Collins, died 1937. Clara Joseph, born 5-18-18.57. Cora Joseph, born 1-23-1859, died 1942, buried Union Cemetery, Georgetown. Samuel R. Joseph, born 1864, rlied 1935 married Lucine Collins, born 1870 , died 1935. Both are buried Lewes Methodist Cemetery.

Daughter of Jonathan Collins. Ethel Joserh, born 8-13-1892, died 11-21-1919, buried Conley's Chapel Cemetery, near Lewes, married Albert Prettyman, son of Wm. H. and Amanda I. I.ingo Prettyman. Ira M. Prettyman, born 7-13-1914 inarried Clarence Reed, born 1919, son of Rufus and Mary (Morris) Reed. Robert Ray Reerl, born 3-14-1941.

Joseph H. Prettyman, born 1917 married Ethaline Steele, born 1917, daughter of Carmel and Estella (Vickers) Steele. Shirley Prettyman. married 1st. Charles Hudson. One Grand Child. 


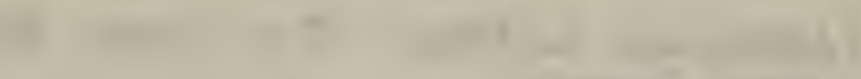

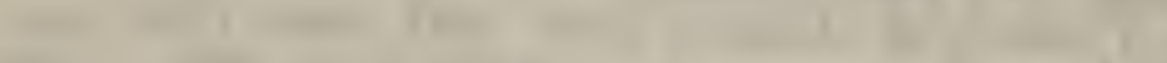

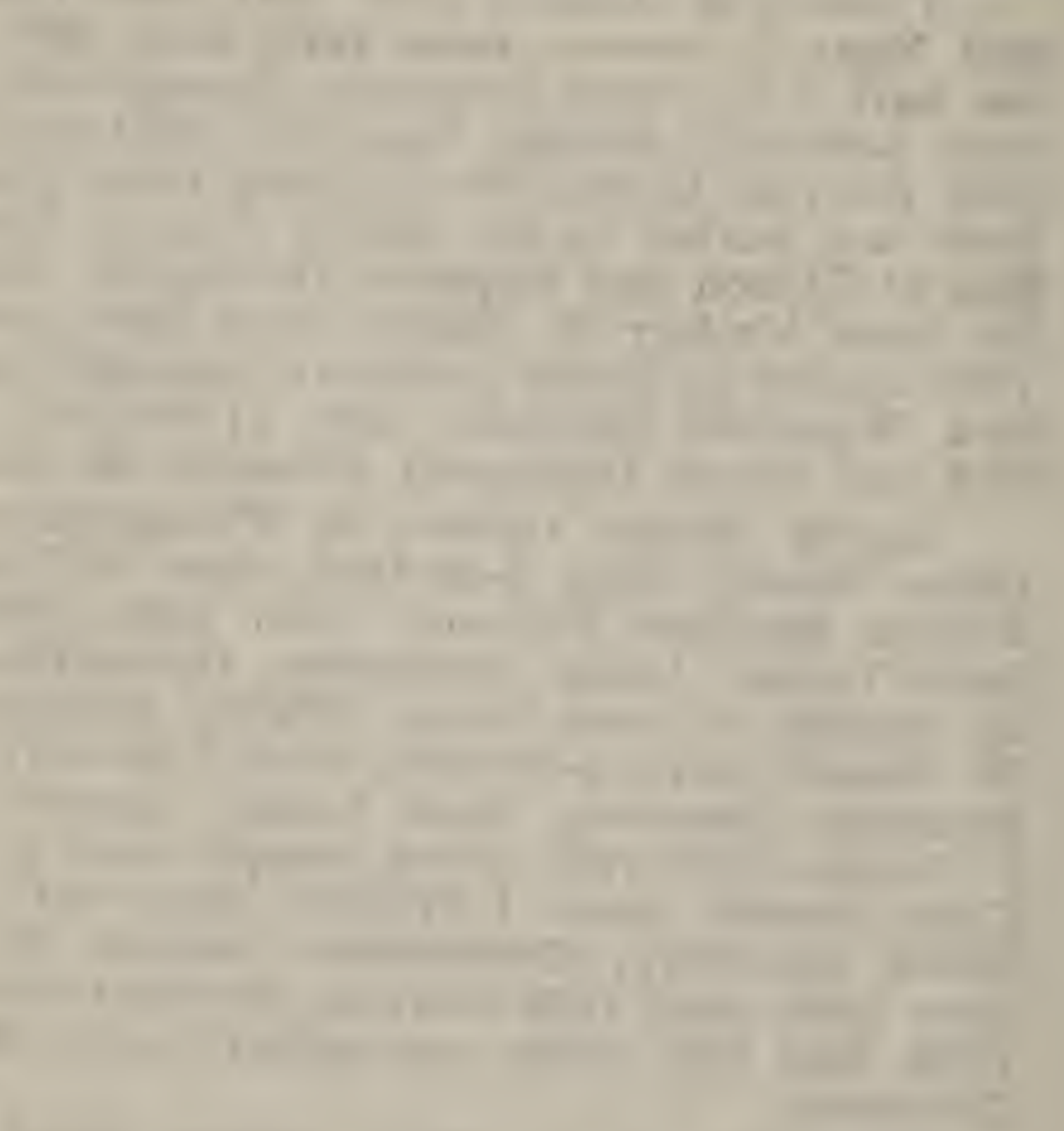

$+15$

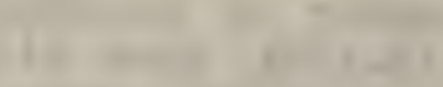

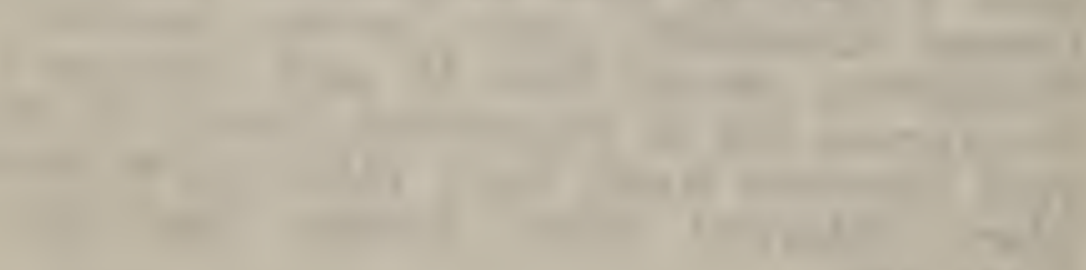
+ at a th

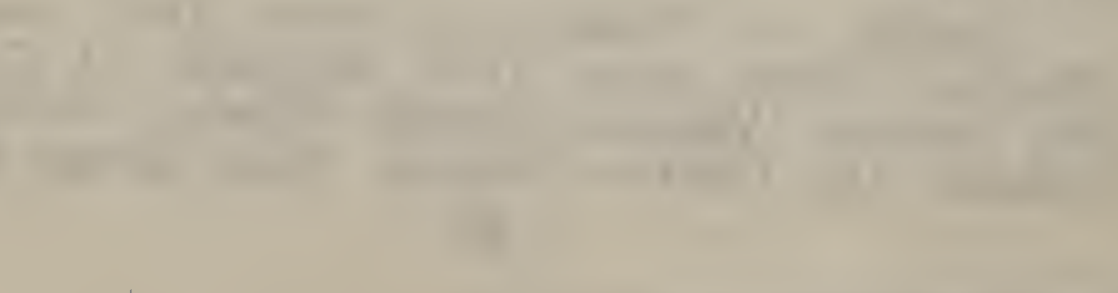


Married 2d. Robert Lawson. Joan Prettyman, born 1040, married Robert Maedler, Jr. Marilyn Prettyman, born 1941. Joseph H. Prettyman, born 1944. Albert Jerry Prettyman, born 1950.

Ruth Joseph, died 1940, buried Lewes Methodist Cemetery, Lewes, Del., married John P. Warrington, son of Kendall J. and Annie Priscilla (Green) Warrington. Samuel P. Warrington married Ida Mae Mears, daughter of Perry and Lela (Warren) Mears. Two children, Ruth Ann Warrington, born 3-29-1959; Stephen Perry Warrington, born 7-27-1961. James D. Warrington married Margaret Wyatt.

Moulton B. Joseph, born 11-27-1984 married Blanche E. Massey, daughter of $\mathrm{Wm}$. and Zilla (Timmons) Massey. Nancy Joseph, born 9-1-1934 married 12-5-1952 Willard S. Burabage, son of Stratton S. and Elsie (Hollaway) Burabage. Debra Ann, born 12-28-1954. Rufus W. 8-4-1939. Emily Joseph married Harry Rickards. No issue. Sarah Joseph, born 7-31-1861, died 2-22-i937 married Thomas R. Walls, born 4-26-1851, died 5-13-1917, son of Asa Walls and Hetty Ann (Walls) Walls. Asa Walls and wife are believed to be buried on the farm owned by (1963) Floyd Wilkins.

Thomas Rodney Walls, Jr., born 7-2-1889 married Lillian May Shaever, born 12-17-1888. Two children. Oscar Walls died in infancy. Ruth May Walls, born 1894 married 1919 Linneaus Prince, 1893. son of Charles and Bertha (Berekert) Prince. One child, Charles Walls Prince, born 1919 married 1st. Lillian Briarcliff, divorced 7-1956. One child, Charles L. Prince. He married 2d. Mary Ann Haight, born 7-7-1931, daughter of William and Verna (Ledcien) Haight.

Peter, son of Jeremiah Joseph. Noah Joseph married 1840 Julian Walls, born 5-16-1819, died 7-5-1884, daughter of Peter Walls. One son, Peter 


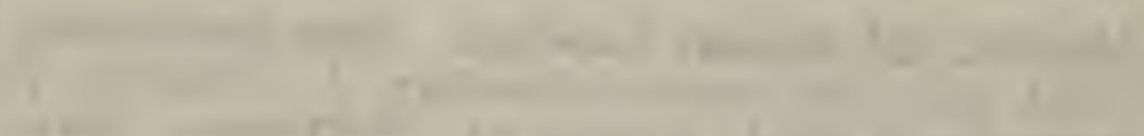

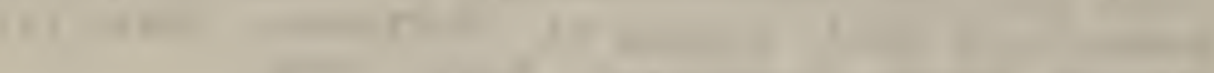

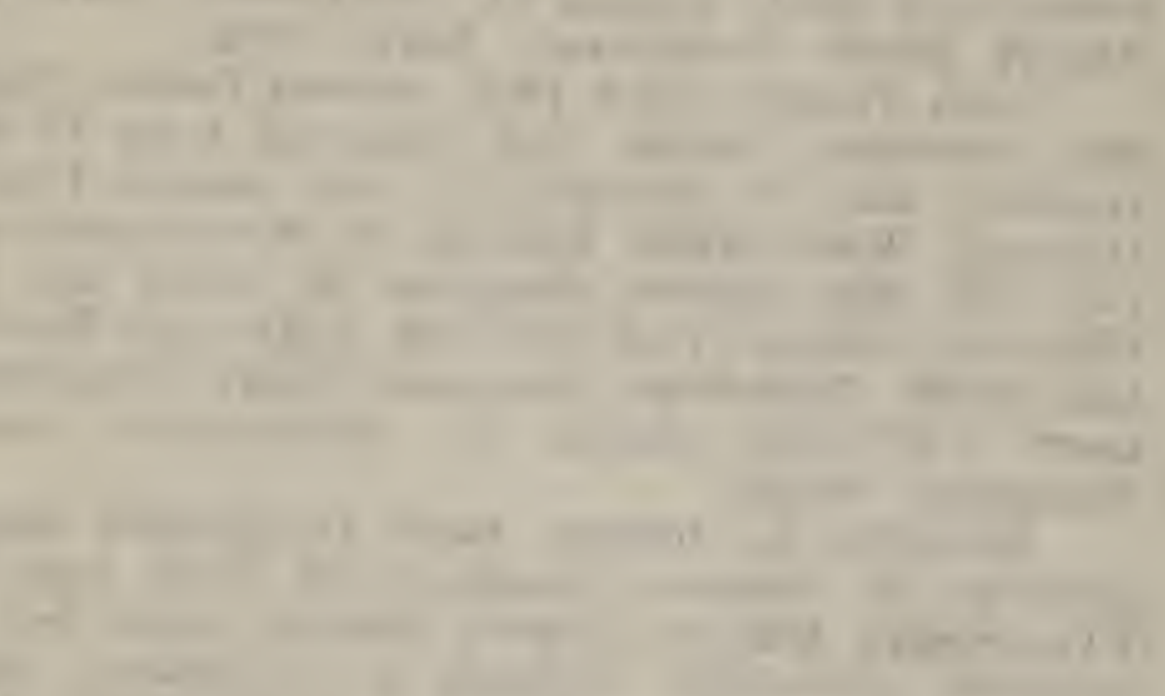
10

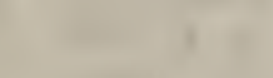

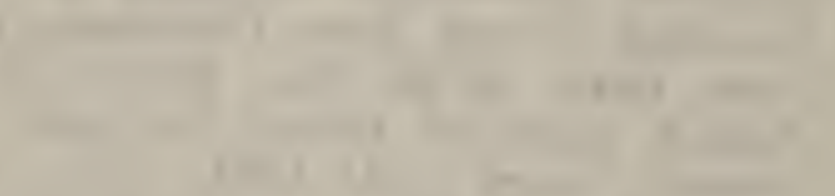

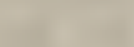

$\sqrt{2}$

10

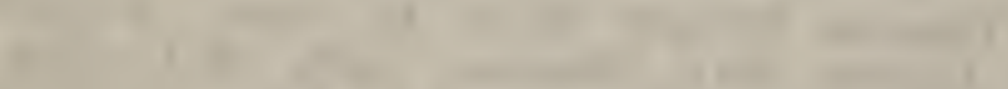

- n

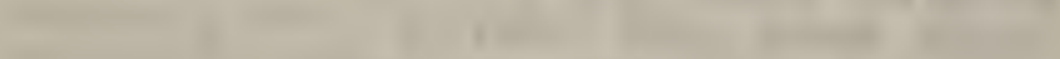

1

-

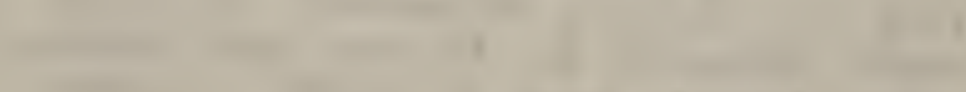

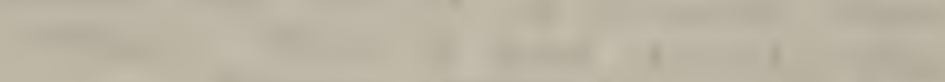

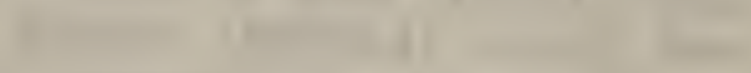

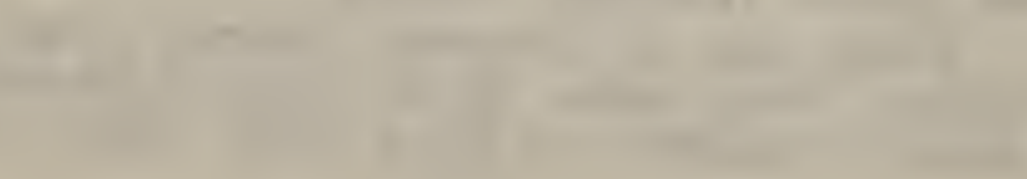


P. Joseph, died 11-22-1894, 47 years old, married Sarah E. L borin 6-18-1858, died 5-22-1887. All buried Union Cemetery, Georgetown.

David Joseph. We believe that he was the son of Wm. and Elizabeth. He was married five limes, but we only know of one, Mariah Laivson. Walter L. Joseph, born 1853, died 1923 married lilzabeth A. ——, born 6-3-1853, died 9-27-1899, Both buried Midway Presbyterian Cemetery. Bessic A. Joseph, born 1884, died 1957 married 2d. Georgc A. Joseph, born 1884, died 19.12. Both buricd Lewes Bethel Methodist Cemetery. Son of liufus and Arcadia (Robinson) Joseph. One daughler, Eclna Joseph married Irving V'ienat. Bessie Joseph married 1st. Henry Mitchell, deceased. One child by this marriage. Henrietta Mitchell married Simmuel S. Williams. One child Betty J. Williams married Randall Knox, Jr. Two children.

Thomas A. Joseph, born 1855, died 1924, married Annie E. - born 1868, died 1898. Three children. Bertha Joseph, born 1885, died 1942, lurlol Mechanics Cemetery, Millsboro married Franli Rogers, born 1891. One daughter died when, voung. Lola Joseph marricd Bivens Day. One inughter Mary Ann Day.

Larry ' $T$. Joseph, born 10-1-1895, died 3-171917, buried Mechanics Cemetery, Millsboro.

Perry P. Joseph, born 1-10-1857, died 1912 marrica Jennie Davidson, born 5-9-1865, died 8-3-19.10. Both buried Megee private Cemetery, Ara Joseph, born 5-29-1881, died 6-6-1953 married J. Scott Brown, born 7-18-1883, died 4-3-1950. Inoth buried in Epworth Cemetery, Laurel. Eight chlldren. Floyd Brown married Meredith Hearn. Flve children. Betty Brown, born 1932, died 5-1610.14, buried Epworth Church Cemetery, married Jennings W. Littleton. One child, Allan Lee. Howard Brown, Donald Lee Brown, Kenneth F. 


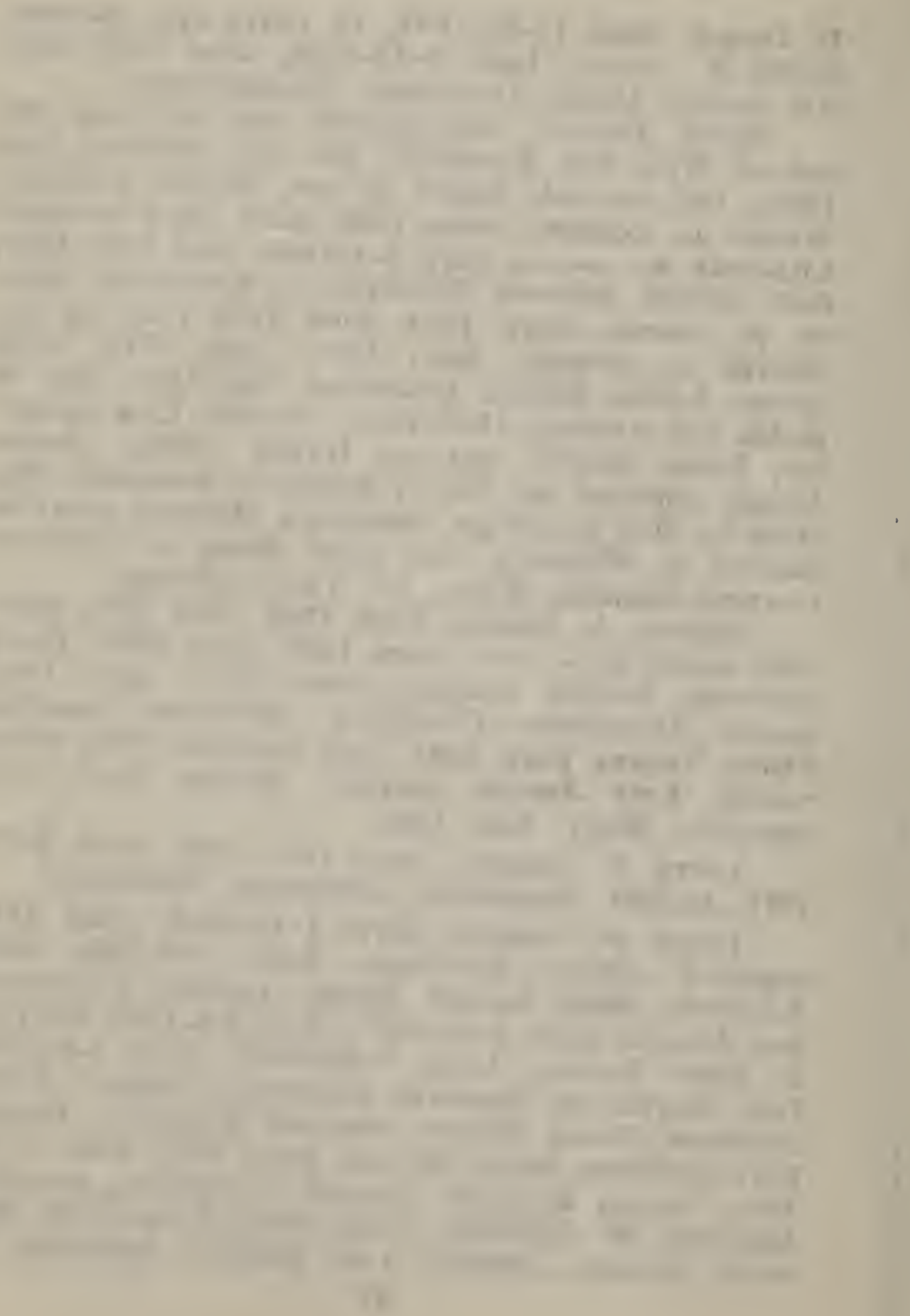


Brown, Arnold W. Brown. John Brown; Preston Brown; Harlan Brown; Sally Brown; Mary; Risby and Flora Brown.

Sally (Sara) Joseph, born 9-8-1884 married 1st. Joseph W. Shockley, born 6-5-1878, died 3-31009. Buried St..John's Cemetery. One son Cürlton Shockley. She married 2d. Maoirn R. Blades, born $1-27-1392$.

William P. Joseph, born 11-17-1886, died 1223-1961, buried Laurel Hill Cemetery, Laurel, Del, Mallied Edith O'Neal.

Catherine Joseph, born 6-12-1889, died 3-11960, buried Epworih Chureh Cemetery, married Ira Brown. Three ehildren. Maude Brown married Wilkersontes. Mildred Brown married

Wilkerson. Lilliam Brown married _-___ Wilkerson.

John Calvin Joseph married Margie Blades, born 1395. died 3-20-1963, buried Epworth Methodist Cemetery, Sycamore. She was a sister to Marion Blades. Melvin A. Joscph. Roland P. Joseph. Edna Joseph married - Rust. She is deceased. Donald C. Joseph. A daughter married Marvil ONeal.

Granville L. Joseph, born 7-12-1895, died 5$9-195.1$, buried Laurel Hill Cemetery, married Litura Baker. Two sons I arry P. Joseph, George A. Joseph.

Larry P. married Catherine Lee. David Samuel Joseph, born 3-10-1900 marilied Clara H.

Norman G. Joseph, born 8-22-1903 married Carrie Legates.

Jennic Joseph, born 6-29-1905 married Davis.

Frace Joseph, born 1-23-1911 married Ben. jamin Venables. Four children, Betty Venables. William B. Venables, Robert Lee Venables, Barala Venables. 


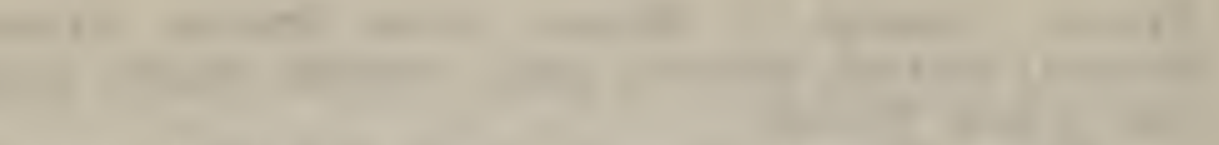

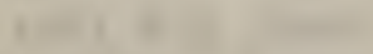

$+1$

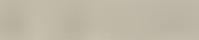

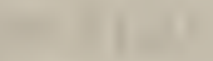

(n)

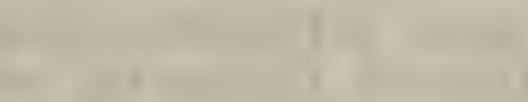

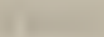

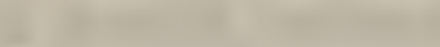

$+1$

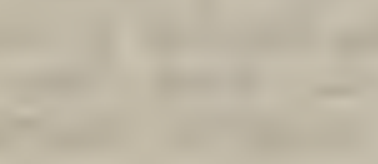

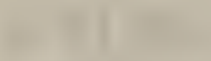

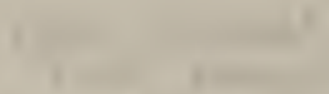

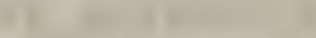

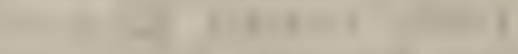

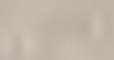

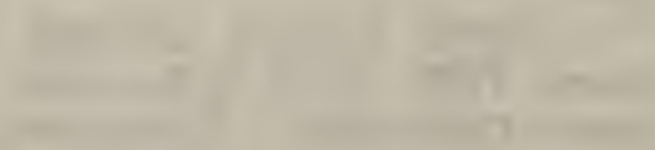

$\sqrt{2}$

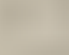$$
\text { 1 }
$$

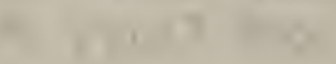

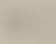

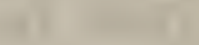

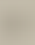


Willam W. Joseph, born 1858, died 1929 marrled Mary A. Howward, born 1853, died 1929. Willlam W. Joseph, Jr., born 1891, died 1958. All burted Union Cemetery, Georgetown. Julius Joseph marrled Flora Hudson. One dalughter Frances Joseph married Paul Bahn, son of Levi Bahn. Howard Joseph unmarried.

Alice Prettyman Joseph, born 5-30-1878, died 2-20-1959 married William R. Ingram, born 3-201870, llied 6-29-1949, son of Thomas R. and Annie T. (Martin) Ingram. One son Riley Hazzard Inarnm inarried Ethel Lewes.

James Joseph married 1st. Annie Copes. Three chlldrun. Arzie Joseph married Frank West. Seven chlldren. Ethel West married _- Shockley. Violn West married - Martha West married - -. Laura West married - lives in Baltimore, Md. Charles West. Walter West. Ida Joseph married - Clevinger. One son Harlan Clevinxor. I.lly Joseph married Tracey Pruitt, deceased. Three sons, Edwin Pruitt, Roland Pruitt, Raymond

Janne Joseph. He married 2d. Cleasie Marahall. Three children by this marriage. Leroy' Skecter Joseph, born 9-20-1896, died 5-13-1956, burled Harbeson Cemetery, married Maggie Mitchcll, daughter of Isah and Sallie Mitchell. Beatrice Juseph married Roy Bunting. No issue. Olive Lea Jasiph married John Armstrong. Three children, Jith Barry, J. Bruce, June Beth.

John Raymond Joseph married Hilda Mitchell, dled 2.29-1943, buried Union Cernetery, daughter of Islah and Sally Mitchell. Catherine Joseph marrled 9-3-1943 Louis Vickers, son of Harley and Thesesa Vickers. Doris Joseph married Walter Hcore. No issue. Raymond Joseph married 2 d. Ievin (Williams) Jester. No issue.

Vilbert Edward Joseph, born 2-22-1892, died 1 $1-2+-1: 49$, buried Hollywood Cemetery, Harring- 

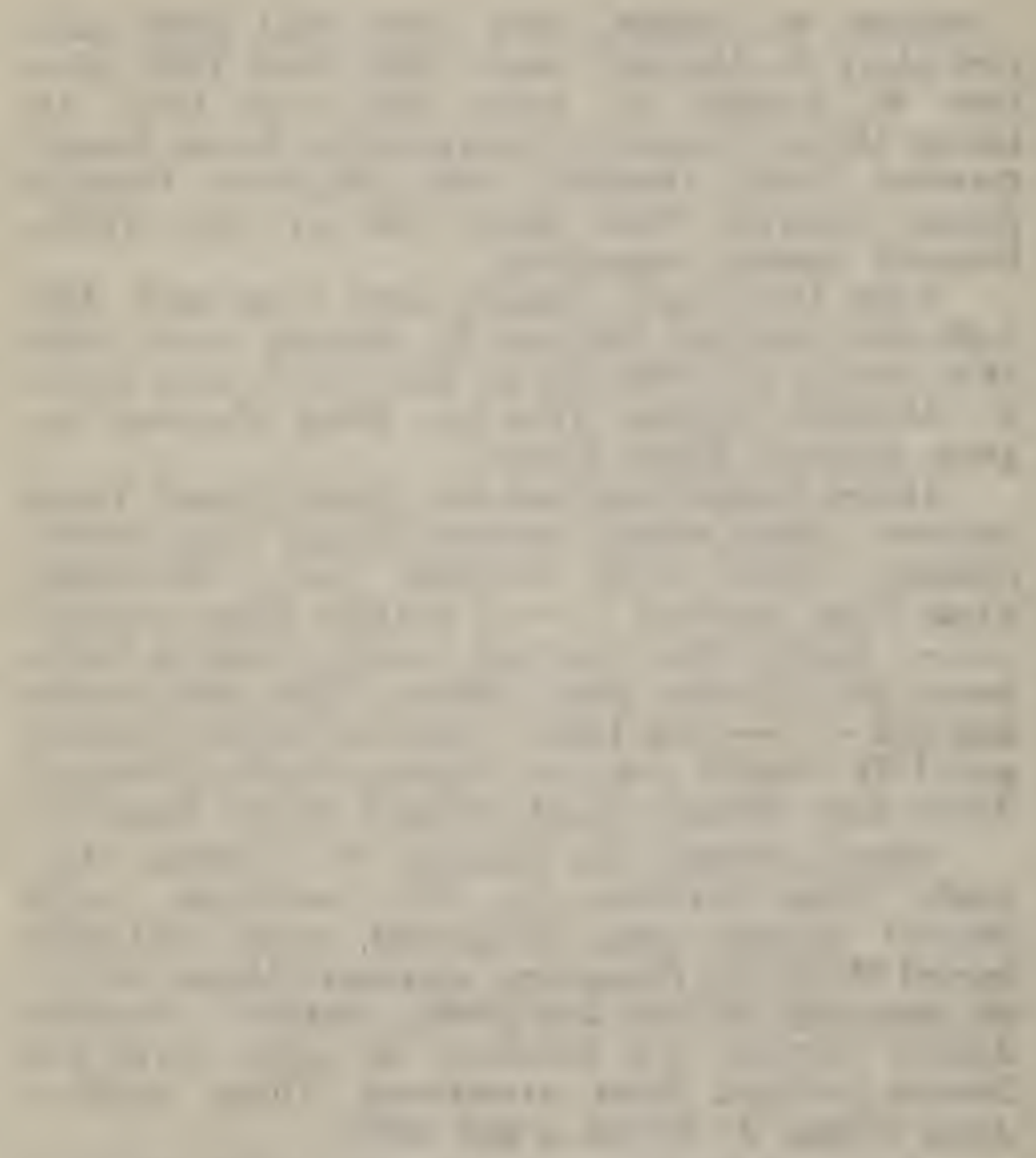

I

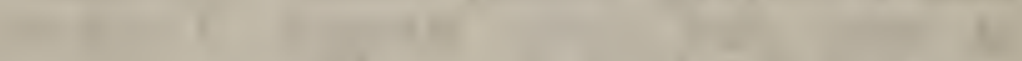

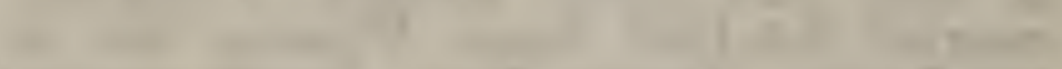

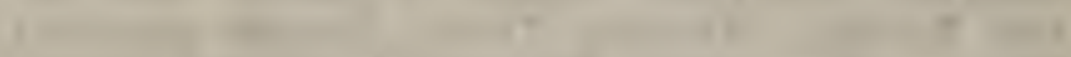

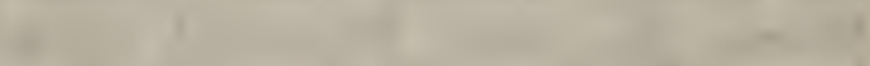
1

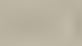
$x^{2}+n^{2}$

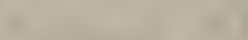
14 
ton, married Edna Anna Beck. Frances Elaine Joseph married Arthur L. Jones, son of Wm. T. Joncs. Two children, Susanne Lee Jones, Thomas Edward Jones. Louise Edna Joseph, born 7-41931, died 11-1-1948 buried Hollywood Cemetery, llarrington.

Mr. James Joseph married three times Mary c.Mgee and had one daughter. Estella Blanche Jose h married William D. J. Reynolds. No issue. Anna Joseph married Manean Hurdle, son of Joseph C. and Eleanor (Walls) Hurdle. Two children. Earl J. I Iurde died in 1961, resided in Baltimore, Md., married Bessie — and had three sons. John Hurdle died in childhood. John Daniel Juseph, born 4-15-1880, died 10-15-1950. buried Mechanics Cemetery, Millsboro, married Mollie Lawson, born 1880, daughter of Jehu and Lydia (Dayidison) Lavison. No issue.

I have been told by $\mathrm{Mr}$. Carroll Wilson that there is another Joseph Cemetery near his home, but we are unable to find it. At one time there were tombs, unt someone removed them. We are sorry about this.

The John Walls Private Cemetery on the farm now owned by (1963) Mr. Charles Moore. Joseph C. Hurdle, born (i-8-1820, died 3-26-1892. Eleanor Hurdle, born 10-10-1826, died 12-10-1889. Harriett Jane Walls, boin 5-31-1834, died 1-8-1866. Theodore E. Walls, died 11-26--, aged 7 years. Ira Crenensurg Walls, died 12-1-1863, aged 5 years. Alfred $P$. Walls, died 12-12-1863, aged 6 years. Lemuel R. Walls, boln 10-8-1842, died 4-10-1886. Harrict Walls, born 7-30-1905. John Walls, born 3-14-1800, died 1-31-1890. Laura Walls, born 3-31840, died 12-5-1915. Eliza S. Hurdle, died 12-8$1879,2: 3$ years. Unnamed girl child, no marker. A farm cemetery on the land now owned by Mr. Ralph Johnson. Peter R. Simpler, died 9-4'- 


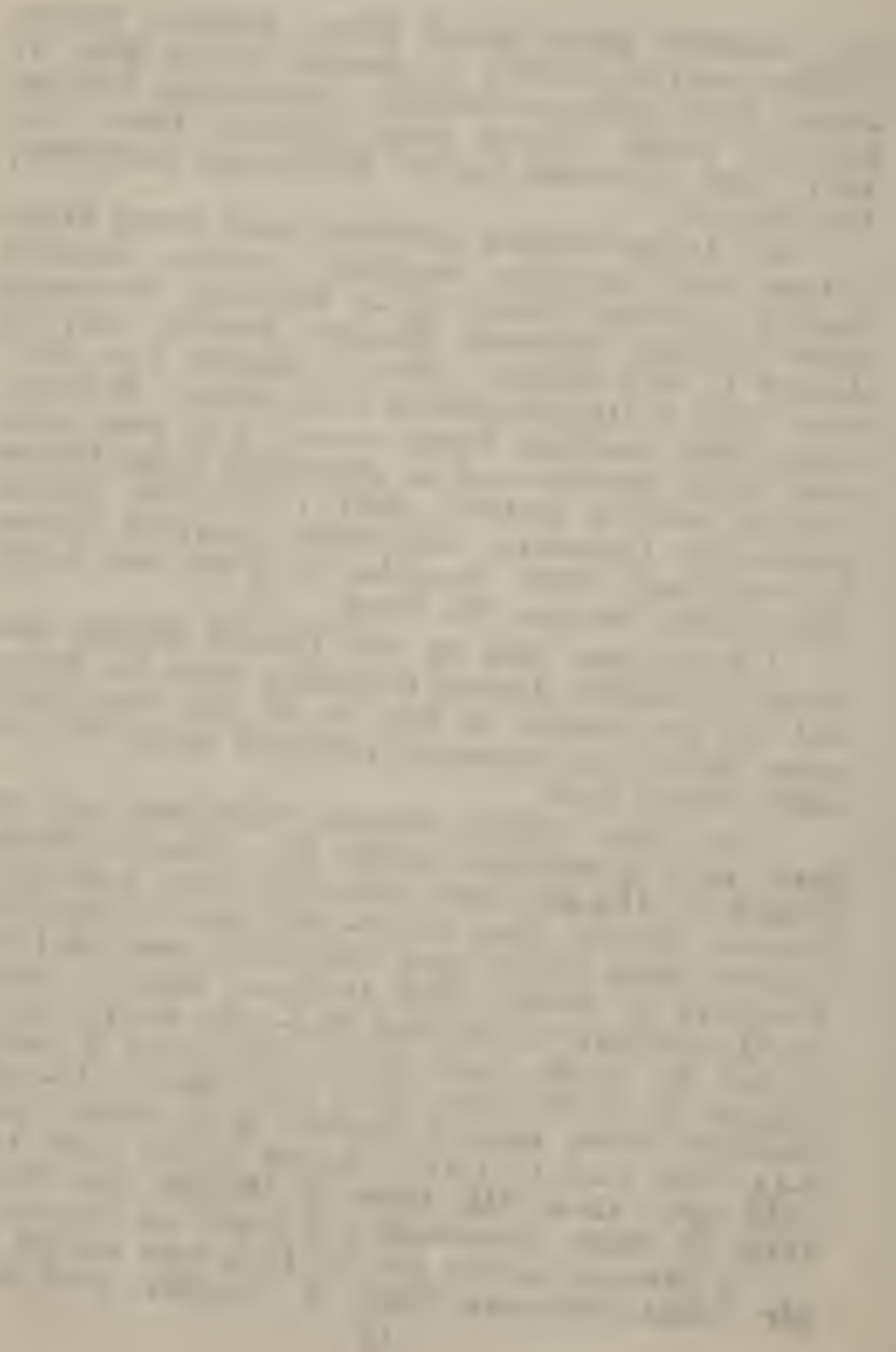


1864,69 years of age. His wife Luranna B. Simpler, died 7-27-1857, 59 years. George W. Simpler, died 6-21-1852, age 3 months. Sarah Simpler, died 110-1859, 36 year's of age. David M. Simpler, died 5-7-1884, 18 years. David H. Simpler, died 10-251855, 34 years. Marquis L. Rogers, born 7-4-1833, died 10-31-1890. Eliza Ann Rogers.

Mrs. Ella Hudson, Mrs. Frank Dodd, Mrs. Albert $H$. Joseph gave this information. Ellie or Elie Joseph. He may be the son of Zackariah and Mary (Walls) Joseph, married a girl, last name of Sines. Zackariah Joseph, born 10-2-1828, died 3-17-1882 married Sarah D. Simpler, born 1-221824, died 10-16-1904. Both are buricd on the Old Green Homestead, Long Neck.

Charles H. Joseph, born 4-13-1847, died 7-211921, married Lydia E. Davidson, born 5-29-1855, ulied 1-5-1924. Both buried Beaver Dam Cemetery, Harbeson. Daughter of John W. and Leah (Green) Davidson. Leah Green was the sister of Hettie R. Green who married Wm. C. Joseph.

Ella Joseph married Stephen P. Hudson, son of Alexander Hudson. Two sons. Paul S. Hudson, $J_{1}$. married Helen - Two children, Diane ay, Michael Allen Hudson. Elwood Hudson married Ist. Clara Walls. One child, Nancy Hudson. Married 2d. Ruth - A child Louis Ann Hudison.

Ruth V. H. Joseph, born 1887, died 7-5-1963 married, John B. Hackett, born 1812, died 19t4. Both buried Beaver Dam Cemetery, Harbeson, Del. One daughter, Mrs. Robert E. Decker. One child.

Gusernie D. Joseph married 1st. Mabel Simpler, daughter of Alfred and Delema (Torbert) Simpler. One daughter —. Married 2d. Ida Joseph, boln 8-17-1899, daughter of Fred B. and Senary (Taylor) Joseph. Esther Joseph married Herman Willey. One daughter, Rebecca Willey. 


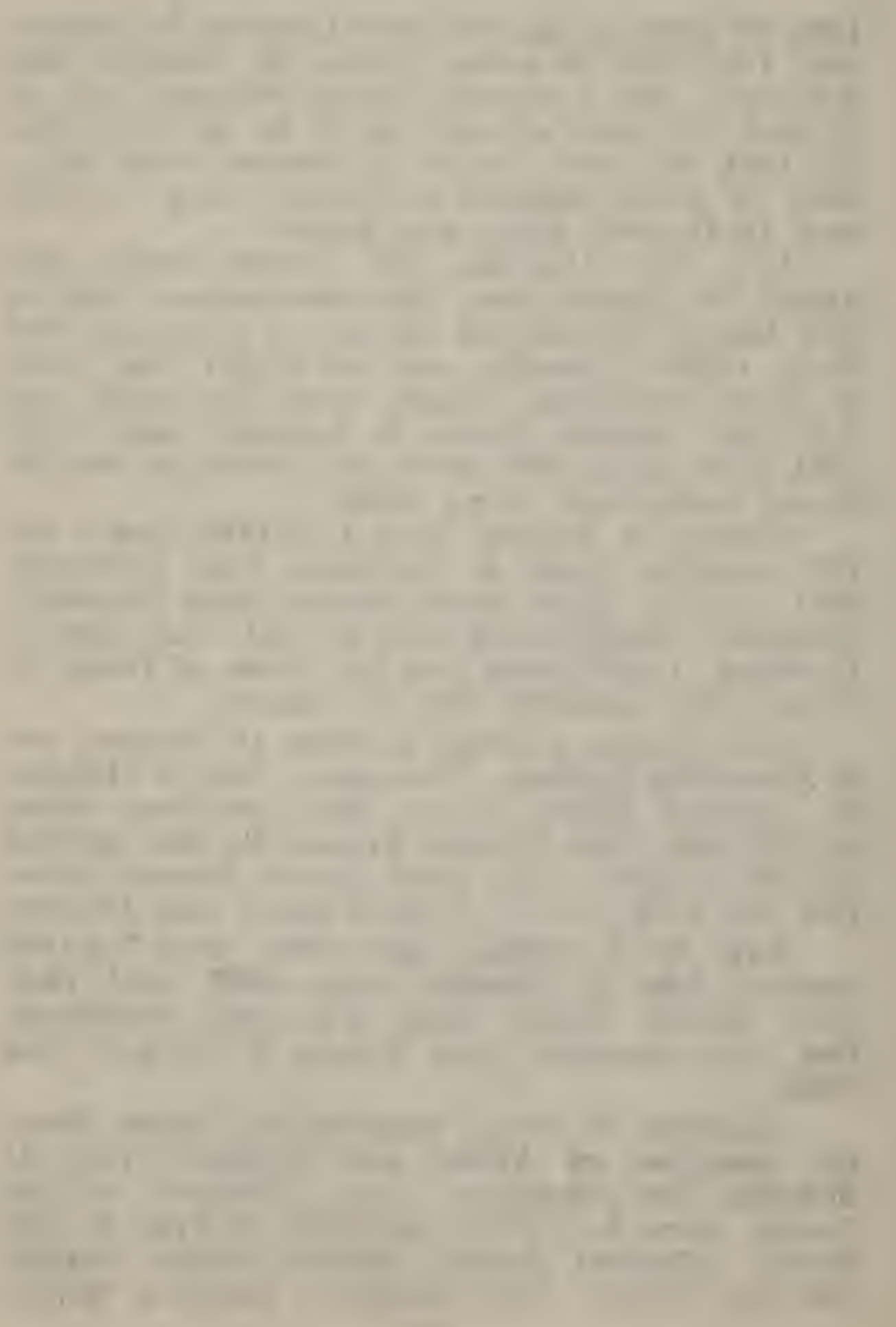


INa Joseph married Herbert Smith. One grandchild. Guernie D. Joseph, Jr., born 1927, died 1929. $\mathrm{He}$ is buried in Beaver Dam Cemetery, Harbeson. Howard Joseph. deceased, married

Effie Joseph never malried. Mildred Joseph never married. Charles W. Joseph, born 10-241896, died 5-27-1915 is buried with his parents at llarbeson Cemetery.

Wesley Thomas Joseph, born 1855, died 1920 marrict Annie Pride. Both buried Lewes Methodist Cemetery.

Virginia Joseph, born 1877, died 1936 married Ebe Lynch, born 1874, died 1916. Both buried Lewes Methodist.Cemetery. 2 sons. Frank Lynch, Norman Lynch, "Fred Lynch, Dorothy Lynch".

May Joseph, deceased, married William E. Wolch. Two children. Mildred Welch married Robert Baily. William E. Welch, Jr. married Florence Toner.

Sarah Joseph married Job Derrickson.

Clarence W. Juseph, born 12-21-1884, died 6-22-19.13. buried Lewes Methodist Cemetery married Hettie P. Himzard, born 1885. Haruld R. Joseph, born 8-2-1910 maried Catherine Wilson, daughter of Albert and Cindy Wilson. Two children. Laura and Clarence Joseph.

I Theodore Joseph married Gertrude Murrey. Two children. James $\mathrm{T}$. married Carol Martin. 'Twr chile'ren Cindy and Robert Joseph. Ruth Joseph. Charles D. Joseph, born 8-18-1920. Unmarried.

I aura Joseph married Linden (Pete) Murray. No issue.

Anna Bell Joseph, born 1892 married Waples Paynter, born 1895, son of William C. and Winona W. Paynter. Two sons, Waples Paynter, Jr., William Wesley Paynter, born 1914, died 1958 buried l.ewes Methodist Cemetery. 
Alfed L. Joseph, born 10-1897 married KathLeyn Morris, daughter of John Wesley and Marcarel Morris. Alfred L. Joseph, $\mathrm{Jr}$. married Cad. ase Whiltington. Three children, Charles A. Joseph, born 8-16-1955; Paul Andrew Joselsh, born 1-219j8; Susanna Joseph, born 1-11-1960. William Fm. Thomas Joseph, born 5-8-1937. Unmarried. Willian D. Joseph, born 1893, died 2-12-196;3, burial Odd Fellows Cemetery, Lewes, married Florence B. Campbell daughter of Goorge Campbell. Two sons, Everett Joseph, Carton Joseph.

Cornelius Franklin Joseph, born 2-13-1859, dled 8-7-1935 married Amancla Prudence Warringlon, Lorn 7-2-1867, died 1-8-1954. Both buried Iawes Methodist Cemetery. Daughter of Kendall J. and Rachel Ann (Wilson) Warrington.

Rachel Ann Joseph, born 1214-1885, died 5\$-10.10 married 1-6-1904 John Lee Brittingham, torn 1380, born 1957. Both buried in Bethel Methodut Cemetery, Lewes, Del. The following received from Mr. Ebe L. Joseph of Smyrna Del. John Le Brlttingham married Rose Margaret Tisher, daughter of Robert Tisher. Three children. Alton Inrlllngham married Dorothy Halloway. Two childeren. Pattie and Alton, Jr. Margaret Brittingham mnrried Gilbert Cooper. Three children. Gilbert Byrd Cooper married Phyllis Johnson, daughter of Clltford and Lika Johnson. Sarah Brittingham, mm 1916, died 1934, buried with her parents in Inwes Methodist Cemetery. Mary Brittingham marred 1st Phillip Lombardo. Married 2d. Wil. llam Taylor. One daughter Dayn Tavlor. Ruth Ullllingham married 1st. Victor Hill. Married 2d. Filwnrd Smith. Franklin Brittingham married Milired Cook, daughter of Frank Cook.

Arthur F. Joseph, born 3-20-1888 married 1F-Im! Sarah (Sally) Stcele, dauchter of James ind Rose (HIastings) Steele. Albert Hagen J. mar- 
1

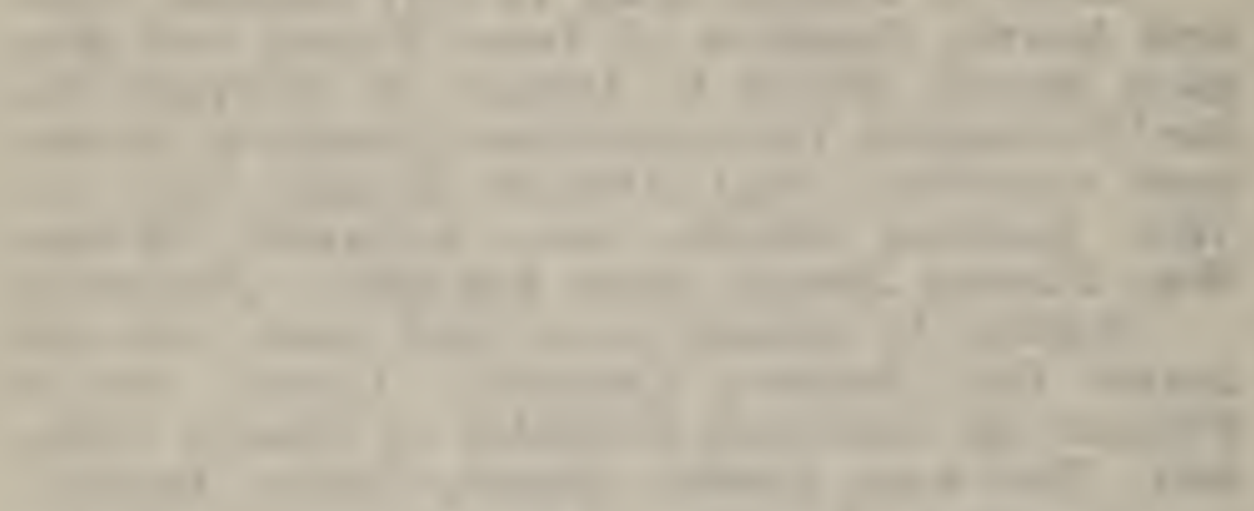

ant

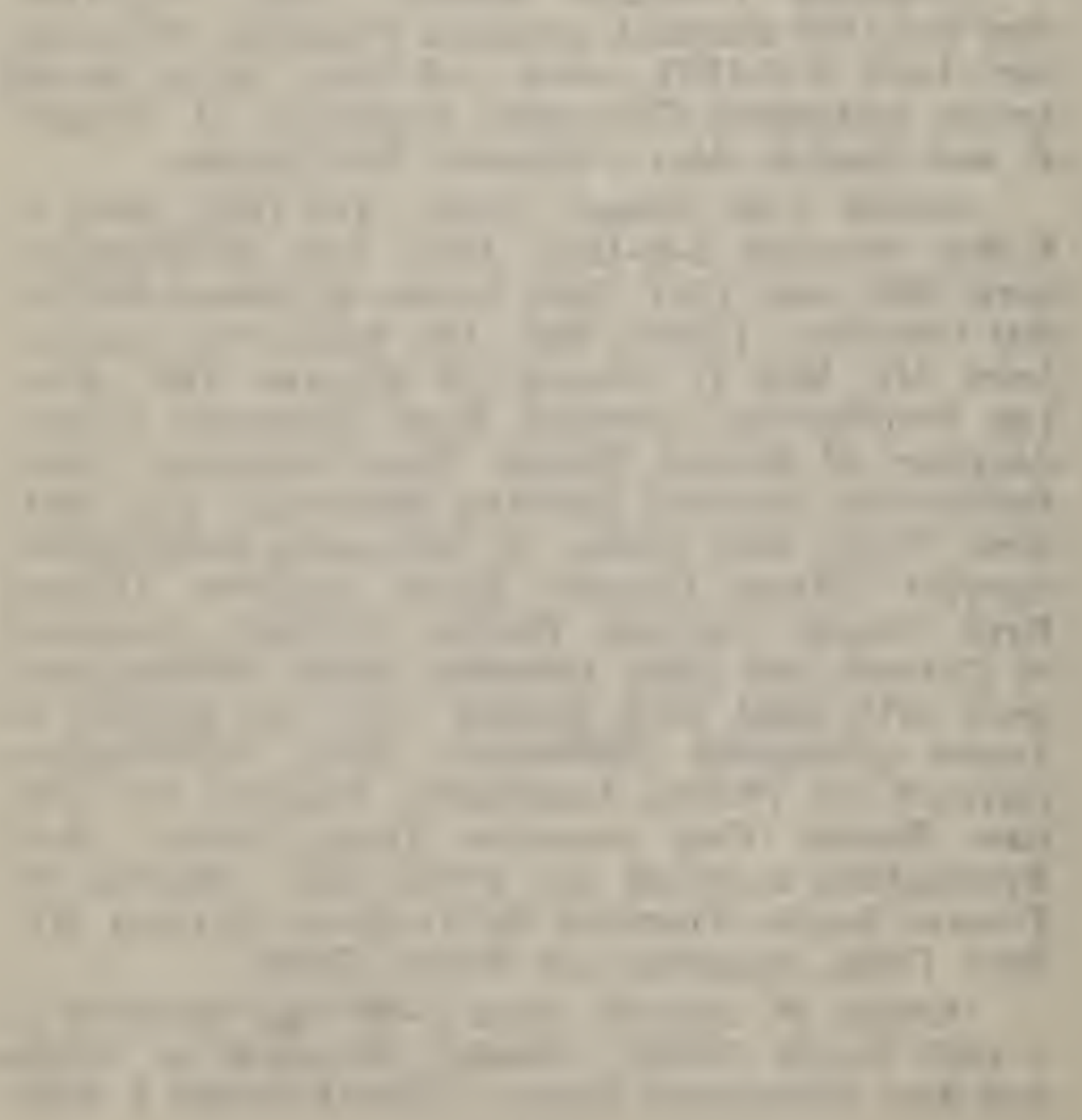


ried Beatrice Irene Hazzard. Douglas A. Joseph, born 12-31-1946. Mildred Joseph died.

Albert H. Joseph, born 11-25-1890, died 5-231950 married 1st. Fannie H. Phillips, born 1889, died 1938. Both buried Lewes eMthociist Cemetery. Two children, Norman H. Joseph, 1918-1934, Helen K. Joseph, 1916-1917, buried in the same place. He married 2d. Isabel (Blackstone) Melson, daughter oi Ernest Blackstone.

Amanda P. Joseph, born 1-14-1893, died 7-219三0 married 12-3-1911 James Ralph Dodd. Two daughters. Son of Absolom Dodd. Hilda Dodd married Elmer J. Revel, son of Charles and Millie Ann Revel. Betly Revel married Aubrey Cordrey. Three children, Sharon, Tracey and Aubrey, Jr. James E. Revel married Doris Baker. Two children, Victoria and James, Jr. Helen Revel married John Roberts, 6-1t-1963, son of Woodrow and Edna Roberts. Eva Dodid married Hoyt Hazzard. Nancy Hazzord married Edward Dee. Two children, Brencia and Pattie Hazzard. Siriney Hazzard. Mrs. Lottie Howard and Mrs. Eva Hazzand gave in this data.

Sarah Delema Joseph, born 9-17-1894 married 12-15-1913 Thomas Cordrey: Died 5-23-1958. Buried Sunset M. Gardens Cemetery. Albert J. Cordrey married Aliene Taylor. Three daughters, Grace, Joyce, Marlene. Grace married Robert Davis. Joyce married Harold L. Wingate. Marlene married Merrill West.

Ann Cordrey married Andrew J. Sammons, born 190.t, died 1954. Buried Sunset M. Gardens Cemetery near Lewes. Son of Elias James and Rhoda (Jones) Sammons. Three sons. Richard Samnons, born 10-23_- married Beatrice Hignutt, born 9-6-_. 'Two daughters, Linda and Donna Sammons. William Sammons born 8-10-_- married Mary Lou. One son, Wm., Jr. Danny Sammons, born 7-4. 

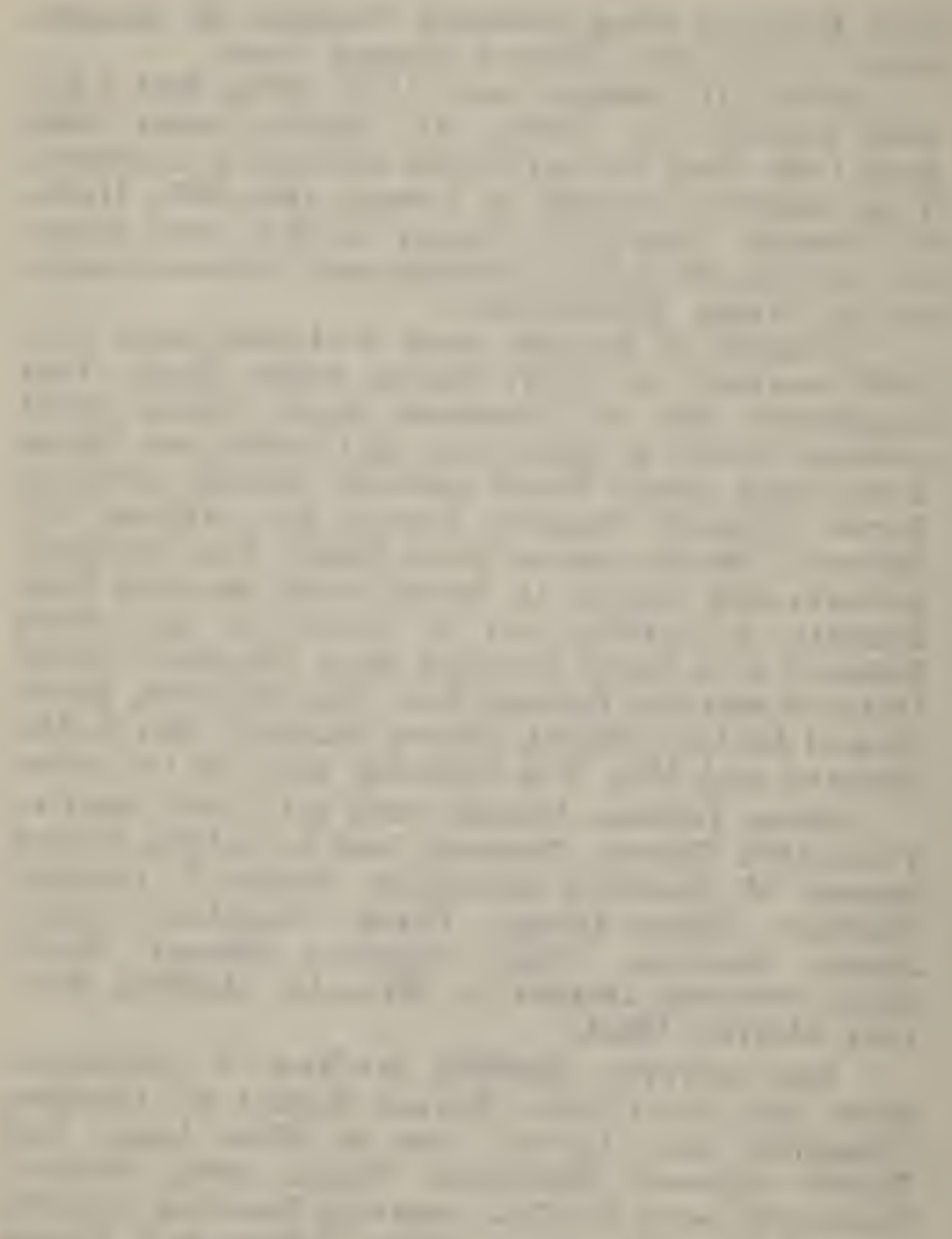

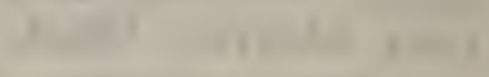

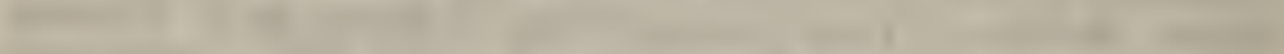
- 
Laura Cordrey married - Foster. Two daughters, Joe Ann Foster. '- in married Howard Sherman. One daughter, Karen Sherman. Mr's. Albert J. Cordrey gave in this information.

Naomi Ellen Joseph, born 10-17-1896 married 1st. John Wells. Ira Wells married Mildred Schroeder, daughter of Reinhart and Alice (Downs) Schrieder. Helen Wells married John D. Lutes. Judith Ann, born 7-28-1946; Helen Marie, born 8-22-1950; Patricia, born 3-17-60. Naomi married 2d. Frank W. Dodd, 9-25-1926, son of Absolom Dodd. Joseph Dodd married Joe Ann. Coard. Jean Dodd married 1st. Joseph Campbell. One girl, Penny Lee, born 8-18-1949. Married 2d. Phillip Cox. One son, Phillip, Jr. The following received from Mrs. Fred Mitchell.

Olivia Hannah Joseph, born 7-24-1900 married 11-1-1919 Fred E. Mitchell, born 7-29--, son of Jacob Mitchell. Granville J. Mitchell, born 5-10- married 1st. Adele. One child, Joyce. Married 2d. Ruth E. Griffith. Two children, Glenn ard Allen Mitchell. Mark Elwood Mitchell, born 727-- married Doris Baker. Three children, Mack E., Jay D., Bonnie May. Elizabeth Mitchell married J. Carl Clendaniel. Two children, J. Carl, Jr., Louise Ella. Fred R. Mitchell married Phyllis Parkhurst, daughter of Fred and Kathryn (Barker) Parkhurst. Three children, Debbie L., David R., Richard Allen. Bessie M. Mitchell married Thomas I. Moore, born 11-30-_. Two children, Karen Rene, Elaine Moore. Mildred Mitchell married Howard Workman. No issue. Catherine A. Mitchell married Marion Taylor. Three children, Marion Wayne, Dickey, Betsey Jean.

H. Ethel Joseph, born 7-27-1902 married Joseph Marsh, 12-17-1921. Richard Marsh married Elva Campbell. Two sons, Dickie, Sammy Marsh, Helen Marsh. Ebe L. Joseph Sr. married 1933 

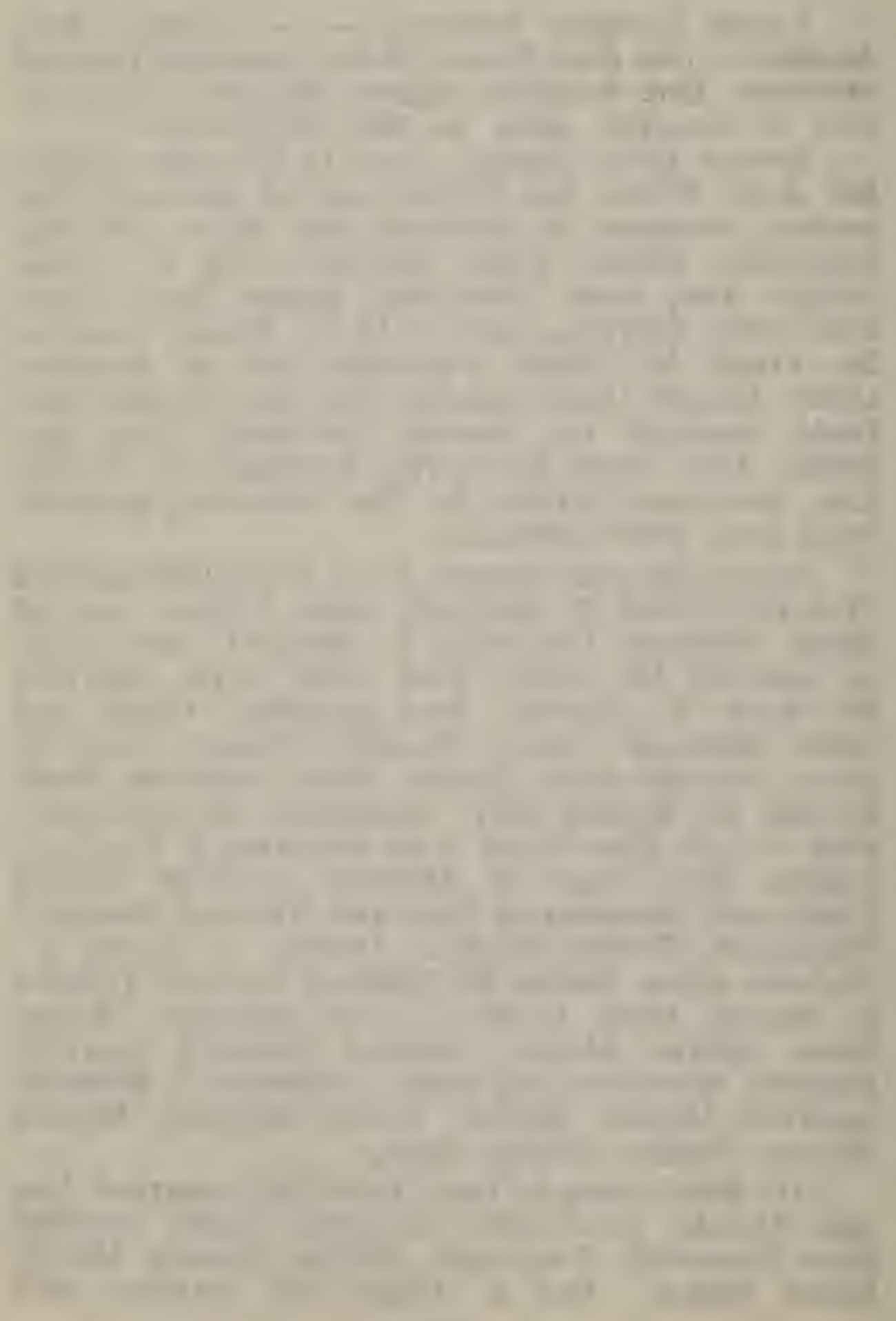
Mabel Arleen Bryant. Ebe Lynch Joseph Jr. married Mary Lou Verdon. Three daughters. John W. Joseph, William Murray Joseph, boln 319-1907 married Elizabeth Blizzard. James and Matilda Andrie Blizzard. No issue.

Lottie S. Joseph, born 11-24-1910 married 63-1939 Charles Martin Howard. Charles Howard married Lincla Echols. One son, David Howard. Cierald Howard. Olaf Howard. Ronald Howard. Mrs. Lottie Howard gave in the information.

Son of Ellie or Eli Joseph. Theodore W. Joseph, born 1836, died 1905, buried Epworth Methocist Cemetery near Laurel married 1st. Emily" Walls. Coulter H. Joseph, born 4-23-1863, died 6-17-1936, buried Concord Church Cemetery near Seaford married Mattie Tarr. Samuel L. Josepty married Helen Lloyd. Martha Joseph deceased. Murried James Jones. Alice P. Joseph married Limmored l. Erown, born 1895, died 10-12-1963. Buried Silverbrook Memorial Park, Wilmington. Thomas E. Joseph married Ruth Wilson. Ralph F. Joseph, born 1905, died 8-13-1963. Buried Silverbrook Memorial Cemetery, Wilmington married -_. Three sons. Coulter R. Joseph. Walter E. Joseph. Frank Joseph. Linwood I. Joseph married Dorothy Carey. Mary Joseph married Leroy Carmine. Russel W. Joseph married Etta Hicks. Robert Joseph married 11-21-1956 Joan Elise Wood. daughter of Tarley and Albert Messick Wood. Joe Ann Joseph married Fdivard Clayville, son of Raymond W. Clayville. Elizabeth Joseph married - Seward.

Rhoda Joseph, born 1859, died 1913 married Jefferson Taylor, born 1857, died 1915. Both aro buried at Concord Church Cemetery near Seaford. Einily 'Taylor married Tarry White. Oliver T. Taylor married Maggie __- Charles P. Taylor. Cicorge W. Taylor Married Elsie - Lloyd 
(

C

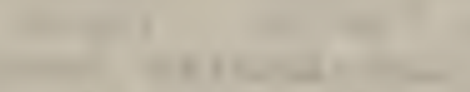

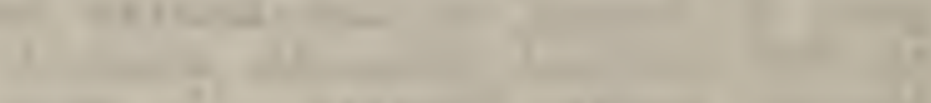

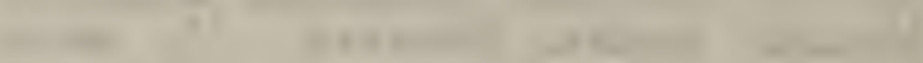

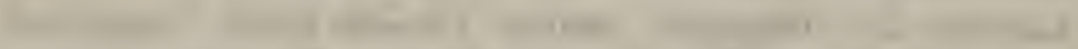

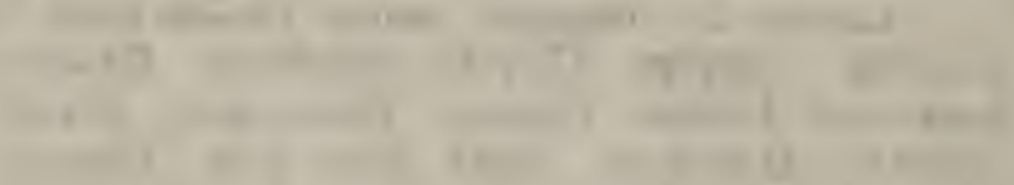

1.

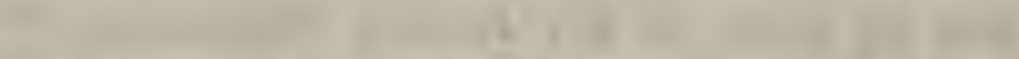

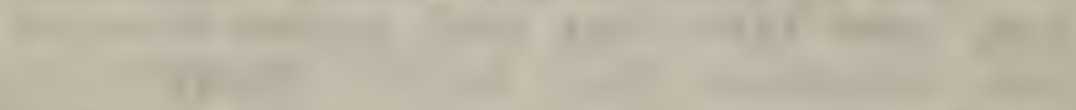

and 4ran
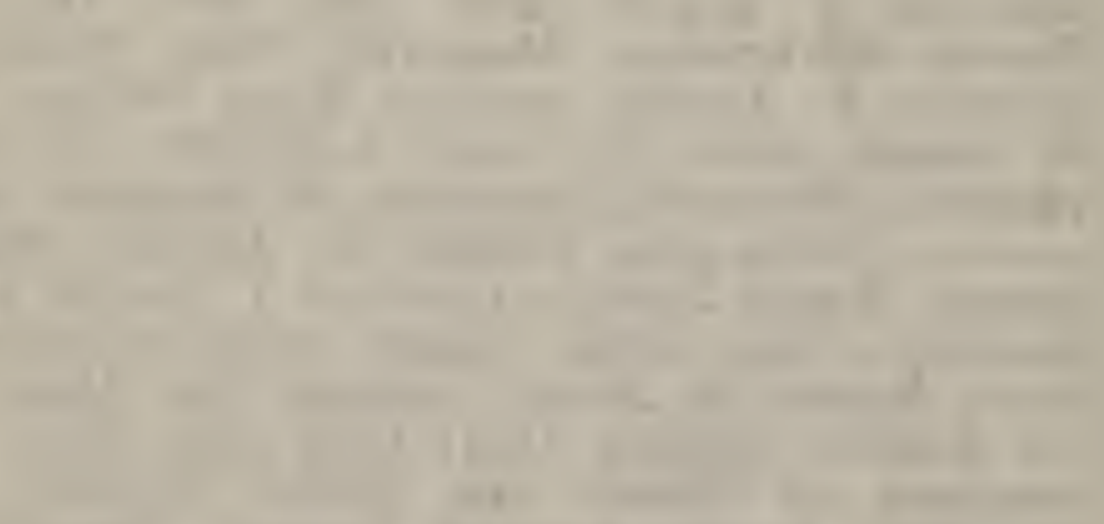

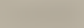

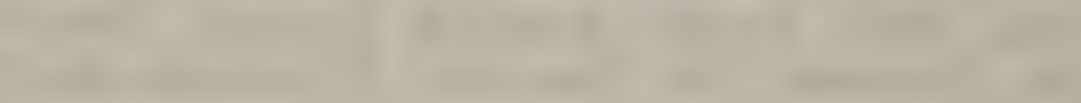

1.

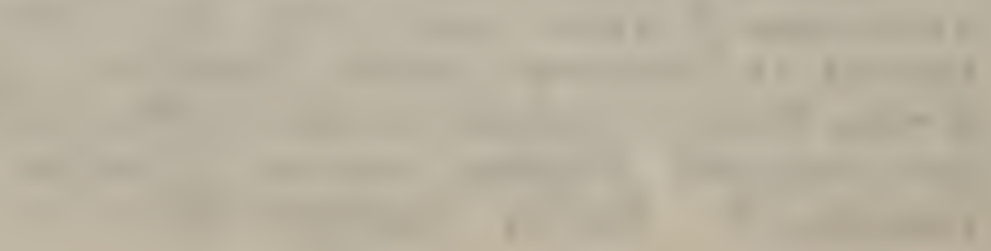


1. Taylor married Maude - Mamie Taylor inarried Harris Warren. Elizabeth Taylor.

IVilliam W. Joseph, born 1-6-1868, died 2-20180y, buried Epworth Church Cemetery married IJu Cooper, born 8-8-1879. No issue. Theodore W. Juseph, Jr., 1870-1948 married Althea Lawrence, worn 1876, died 1950. Both buried Odd Fellow's Cemetery, Seaford. Emma Joseph, born 11-301895 married Alfred L. Rogers, born 9-20-1891. Ullk Juseph. Lawrence Joseph. Clara Joseph. Ainus Henry Joseph married Rose L. - . .

George Joseph died at an early age.

Theodore Joseph, Sr. married 2d. Katie Allen, born i86 t, died 2-30-1905. Buried Epworth Cemelery. Elmer John Joseph, born 7-17-1880, died 11-8-1917 buried Blades Church Cemetery, marreal 11-15-1898 Ida Mae Cooper, born 8-8-1879. The widow of William Joseph. Etha Ellen Joseph, worn 10-13-1900. Roy Lee Joseph, born 1-16-1903. Bertha Mae Joseph, born 8-13-1905. Mabel Delema Jaseph, born 7-21-1912. Howard Monroe Joseph, born 1-23-1916 married Esther Nogel. Hilda Marie Joseph, born 8-7-1923.

Daniel Joseph, born 1-17-1883, died 1947 marned Betty Richardson, deceased. Minnie Joseph. Walier Joseph, Raymond Joseph. Charles Joseph, Frances Joseph, Daniel Joseph, Jr.

Addle Joseph, born 4-20-1885, died $1960 \mathrm{mar}-$ Her Cicorge Lloyd deceased. Two children, Helen R. Lloyd, deceased, Orlando Lloyd. Addie inarried of. Filmer Foskey. No issue.

Mnggle Joseph, born 7-30-1387, died 1954 marred Nath W. Brown, born 1902. Three children, Norence Brown; Lynden Brown, deceased; Walter Iro:in.

Fifle Joseph, born 7-29-1890. She is the only ono of her family still living. Married Paul Richarrison, dicyl 1946. T'wo daughters, Geneva Rich- 


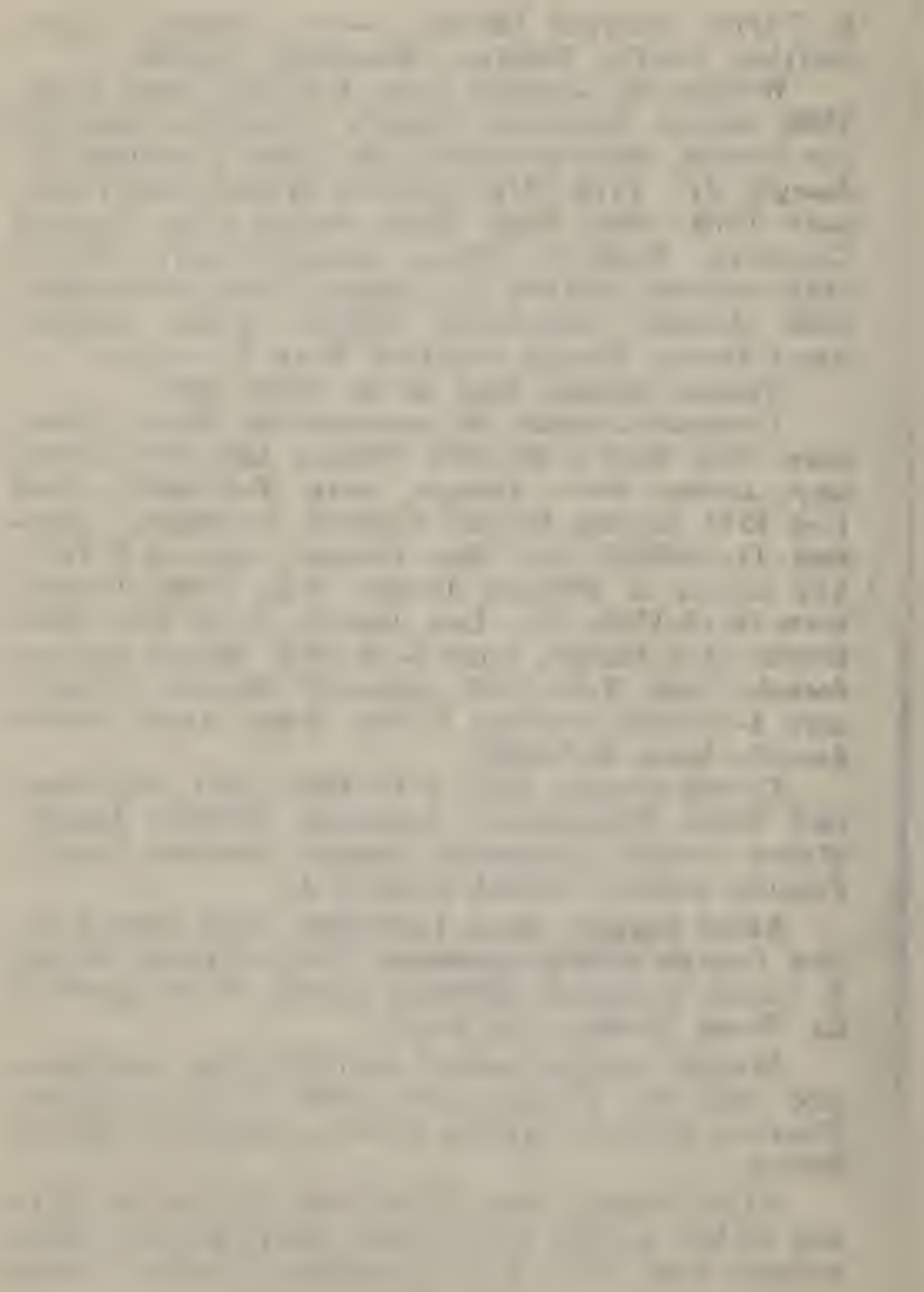


ardson, died 1947, Arlene Richardson.

Lillie Joseph born 5-29-1893, died 1919 married 1st. John Walls. No issue. She married 2 d. Hugh Burtelle, born 1887, died 1931. Buried Odd Fellows Cemetery, Seaford. 'Two daughters, Mildred Burtelle and Sylvia Burtelle.

Franklin Joseph, born 10-30-1895, died 1959, buried in Salisbury, MId., married Elsie Parsons. Mclvin D: Joseph born 1927 married June Mears. Four children, Ronald M. Joseph, L. Elaine, Donald, Charles F. Joseph. Russel Joseph died Buried Arlington N. Cemetery. Married Joan Ida. Franklin Joseph, Jr. married Katherine Tatman. Seven children, Jerry, Janet, Phyllis, Franklin III, Rickie, Eddie, Darryl. Narie Joseph married Bryd Austin. Addie Joseph married Charles Conalway. Victor Edward Joseph married Billie Jean Baker, daughter of John and Lawcinda Sexton Baker. Paul Joseph married Jean Hitchens, daughter of Ephraim D. Hitchens. Kathleen Joseph married Laurd Tatman. Rohert Joseph married

Two children. Sherion May Joseph, born 1948, died 1950. Buried Mechanics Cemetery, Millsboro,

Adelia Joseph, born 7-23-1900, died 1945, buried at Barratts Chanel Cenetery married Benjam in Giuttari, born 189? in Sicily at the toe of Italy. Seven children. Nicholas B. Giuttori, born 4-4-1920 in Pautucket, Rhode Island married Virginia Wixs. She had three children by her first hushand, Virginia lee Holston; Margaret Fleie Ifolston: Norma Holston. Mildred Barbara Giuttari married IIowarel Franklin Ney, Jr. Three children, Bruce Kenneth Ney', Barbara Jane Ney married Samuel I awence Gillespie. Sandra Raye Ney. Sarah Mace Giuttari married 1st. Milton V. Jones, deceased. One son Milton Hunter Jones. Sarah married 2d. Technical Sergeant Gerald V. Dickinson. Catherine Ellen Giuttari married Jos- 

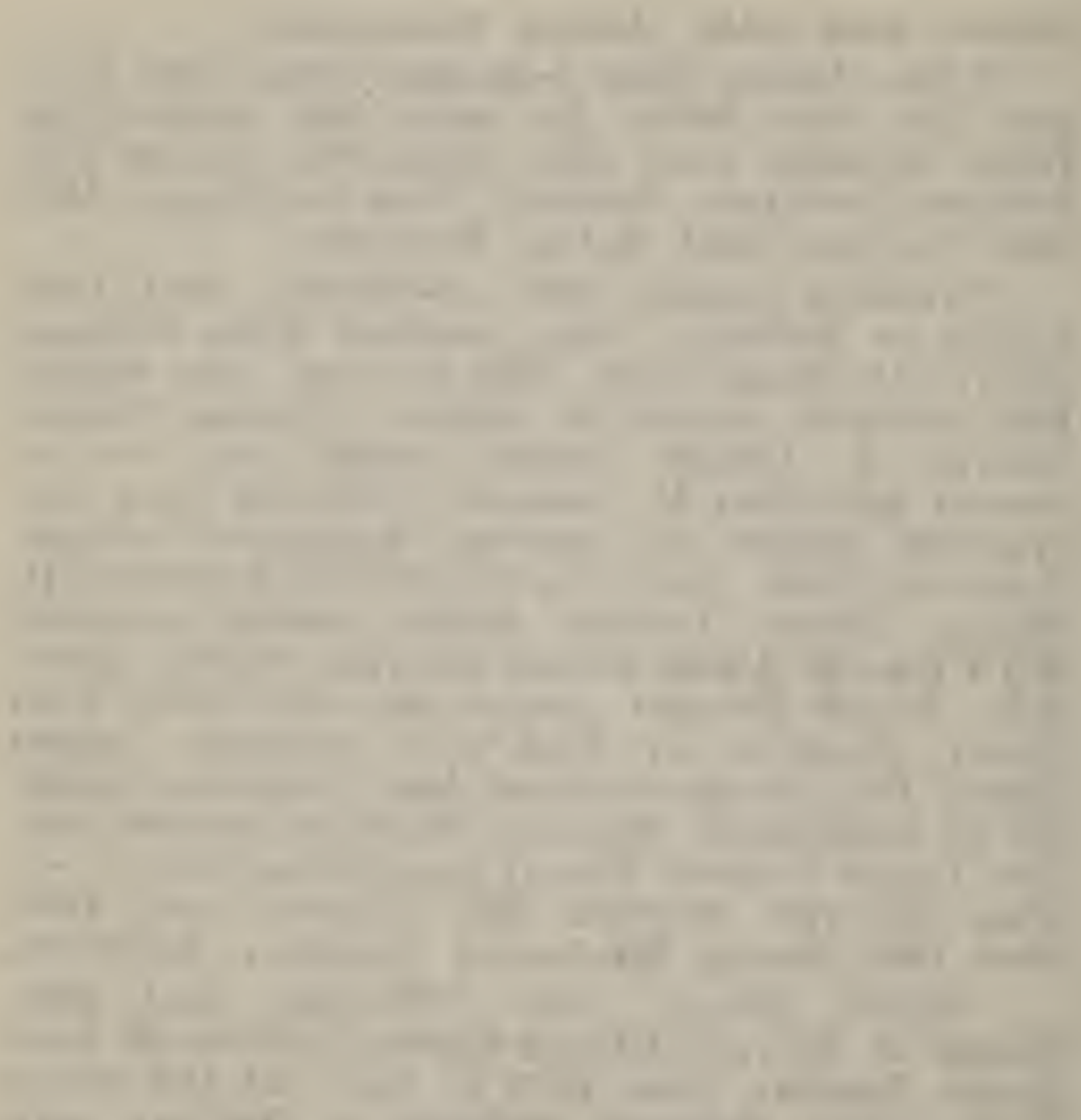

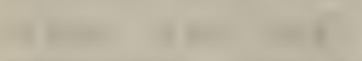

(1)

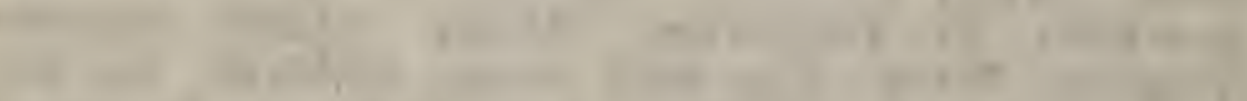

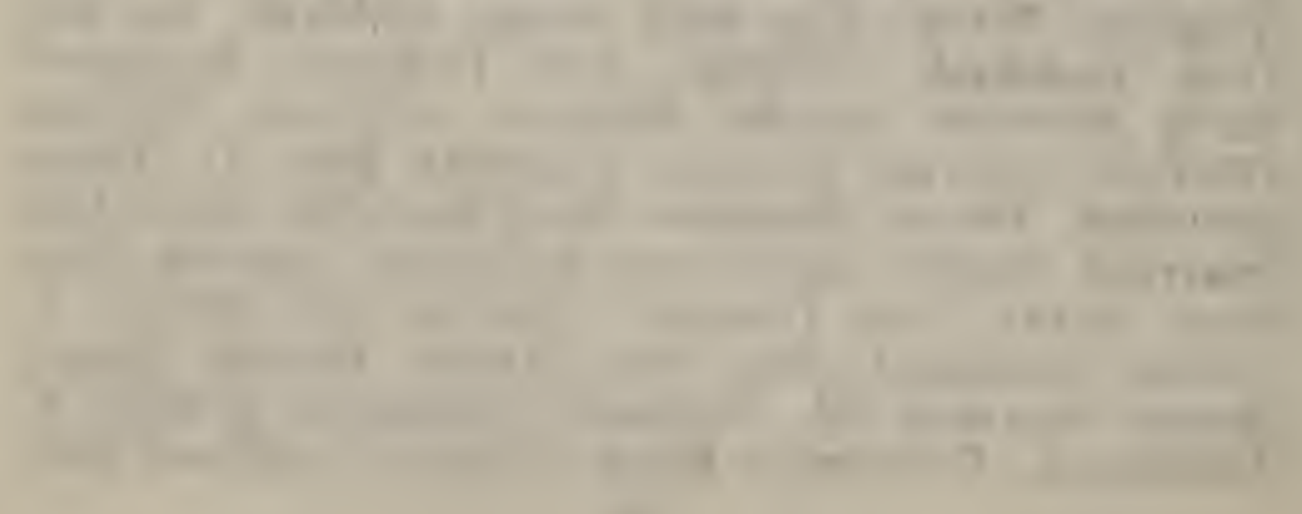
10 
uph Berna of New York. John, Joseph, Steven Vincent, Judy. Evelyn Marie Giuttari married Charles Jackson, Jr. One daughter Susan Jackson. Benjamin Giuttari drowned at Bowers Beach, Del. at the age of 7 ycars. Joseph Giuttari married Christine. Four children, Deuringer B. Giuttari, Joseph B. Giuttari, Franklin J. Giuttari, Diane C. Giuttari.

Fisie Joseph and Adelia were twins. Elsia died five months of age. George Joseph, born 430-1903, died 1925. The following received from Mr. Nicholas B. Giuttari of Dover, Del.

Elihu Joseph married Nancy Pool. Elisha Joseph married Emiline Parsons. Both buried somewhere near Georgetown. One son went out west and died there. Elihu Joseph married Unice One child, Thomas H. Joseph, born 1839, dled 1910, married Rebecca ___. Both are burled in Ebenezer Church Cemetery near Lewes, Del. Fred B. Joseph, born 1859, died 1937, malried 1st. Senary B. Taylor, born 1864, died 1905. Woth buried Asbury Methodist Church on Laurel and Georgetown Road. Orlando Joseph, born 1890, died 1941. Buried Epworth Methodist Centeiery near Laurel. Marrier Cecil P. Elliott, born 1890, daughter of Ben Elliott. Verna E. Joseph, ixurn 3-21-1919, died 12-30-1963, buried in Odd Fellows Cemetery, Laurel, Del., married 1 st.

Boker. 4 sons, Donald E., Marvel J., Robert and Orlando Baker. She married 2d. Norris L. Nibbeth. Pauline W. Joseph married 1st Joe Webb. Marrled 2d. Hammond. Nona M. Josepn marrled Miller. Orlando Joseph, Jr. Raymond Joseph. Neva Joseph.

Moland D. Joseph, born 1-12-1887, died 6-41083, buried at Liurel, Del., married Grace H. Thompson. Anna B. Joseph married Carmenn. Gaston 'T. Joseph, Rudolph E. Joseph. Nein Joseph married — Little. Claude R. Jo- 


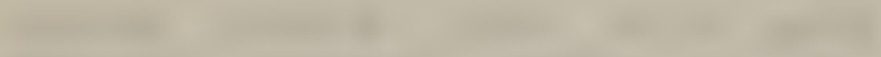

10
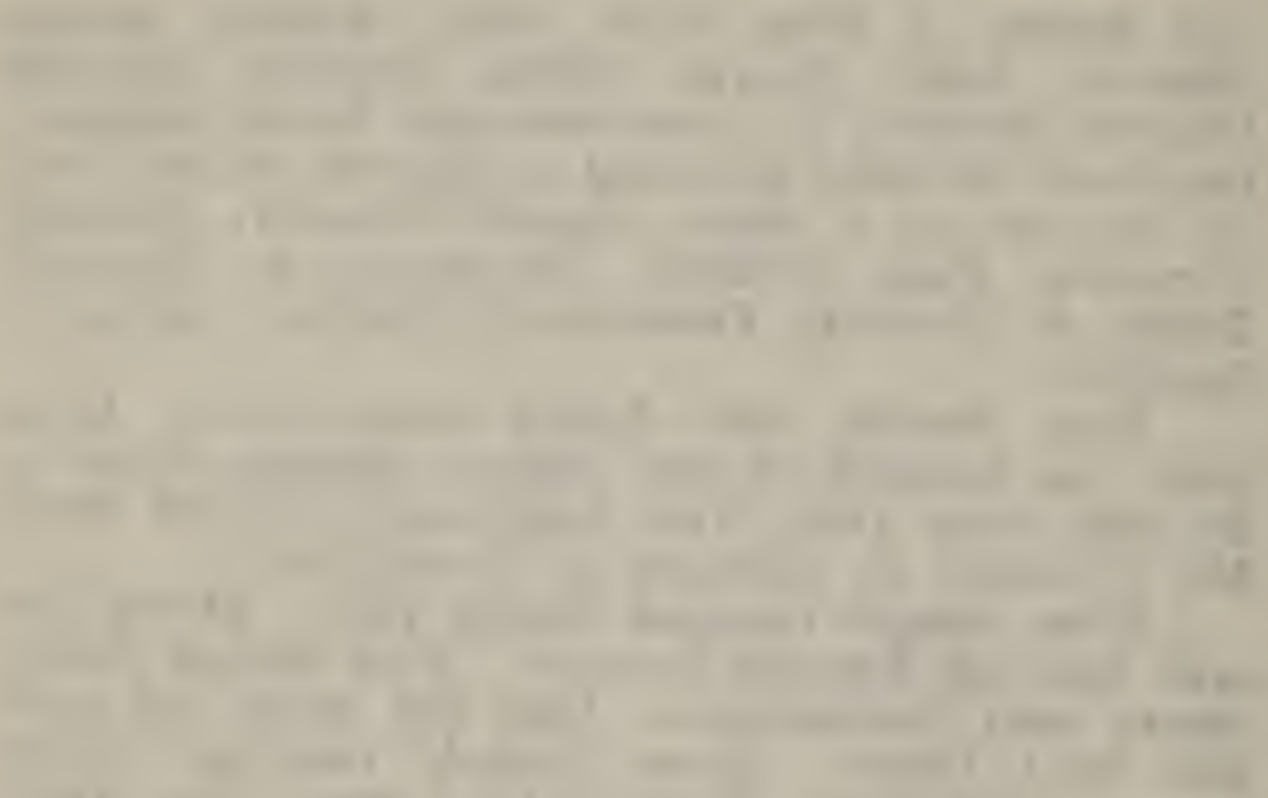

(10.

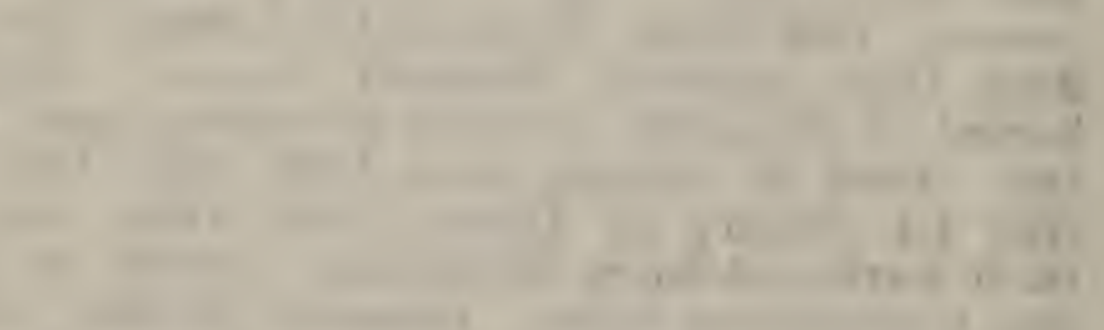

(n)
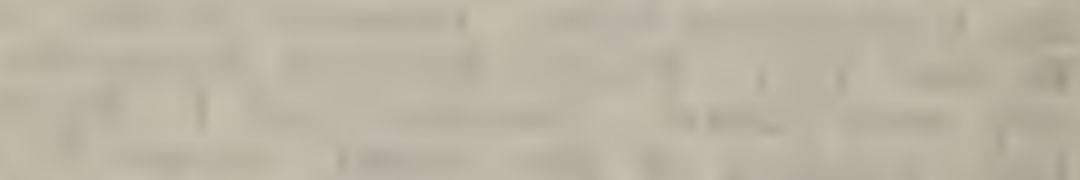

-

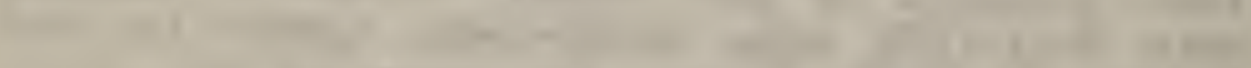

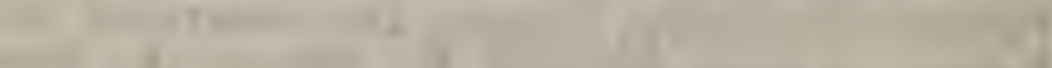

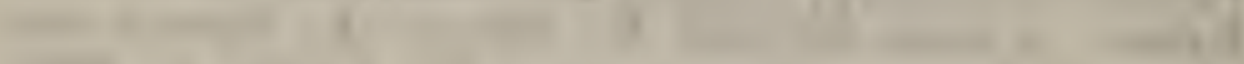

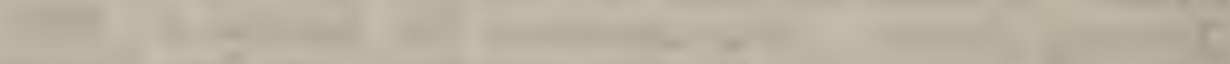
(2)

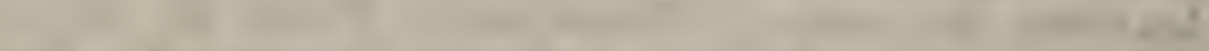
t.

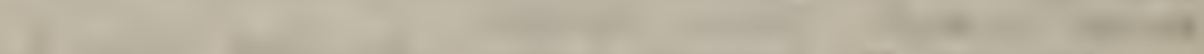

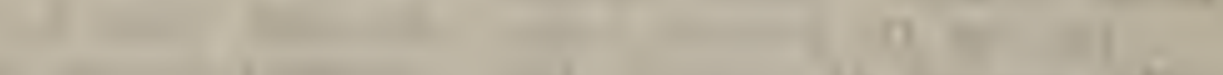

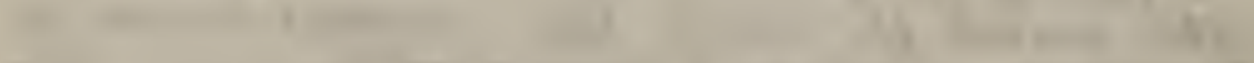

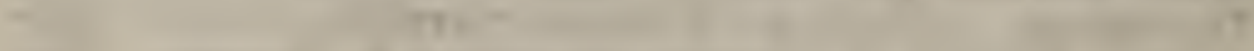

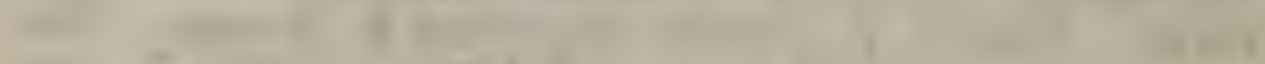

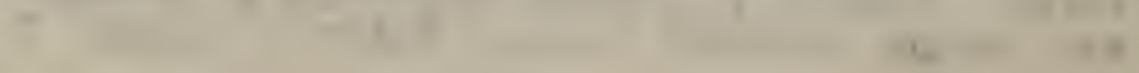


seph. Kathleen Joseph married — DeFelica. Victor Joseph married Lena Hudson. Two children, Mineva Joseph, Elton Joseph. Bertha M. Juseph, burn 5-27-1894, died 9-27-1963, married 1st. 1917 Roy Gumby. She married 2d. 1222-1943 Geo. W. Baker. No issue. Mable Florence Joseph, 2-20-1918, married Clifford Foreman. Roland E. Foreman, born 4-1-1920, married lst Irene Faulkner. One child, Stanley E. Foreman. Marrier 2d. Mary Catherine Chance. Three children, Gayle Foreman, Linda Fureman, and Wayne Foreman. Married 3d. Eva Coleman. Four children, Gregory Foreman, Janice Foreman, Robin Foreman and Roland Terry Foreman. Virginia Lee Foreman, born 1-5-1922, married 1st. 11-12$19+1$ Lester Mueller, died 12-9-1947. One child, Lanry Mueller. Married 2d. William A. Larrimore married 4-30-1949. One child adopted Debra Jean Larrimore, 1-30-1960. Ernest Franklin Foreman, born 4-14-1924, Inarried Edith Mac Daubach. Seven children, Ronald, Ernest, James, David, Jerry, Janet and Richard. Anna Mac Foreman, 10-1-1928, married John Ray Hutchison. Three children, Joan Rae Hutchison, Mary Lois and Lois finn. Florence Marle Foreman, born 1-13-1931, married 10-10-1949, John Edward Chance. Two children, Constance Narie Chance and Cynthia Carol Chance.

Harry F. Joseph, born 6-16-1901, died buried in Union Cemetery, Georgetown, married Nary Bailey, daughter of $\mathrm{Wm}$. I. and Annie $\mathrm{E}$. Bailey. Six childien. Harry Joseph, Jr. married lst Josephine Elliott, daughter of Norval Elliott. One son, Raymond J. Josephine Joseph. Elizabeth Joseph. Linwood Joseph. IIorace Joseph. Onlec Josciphine.

Ida Joseph married Guernie Joseph, son oi Charles H. and Lydia (Davidson) Joseph. This family tree can be found elscwhere in this book. 


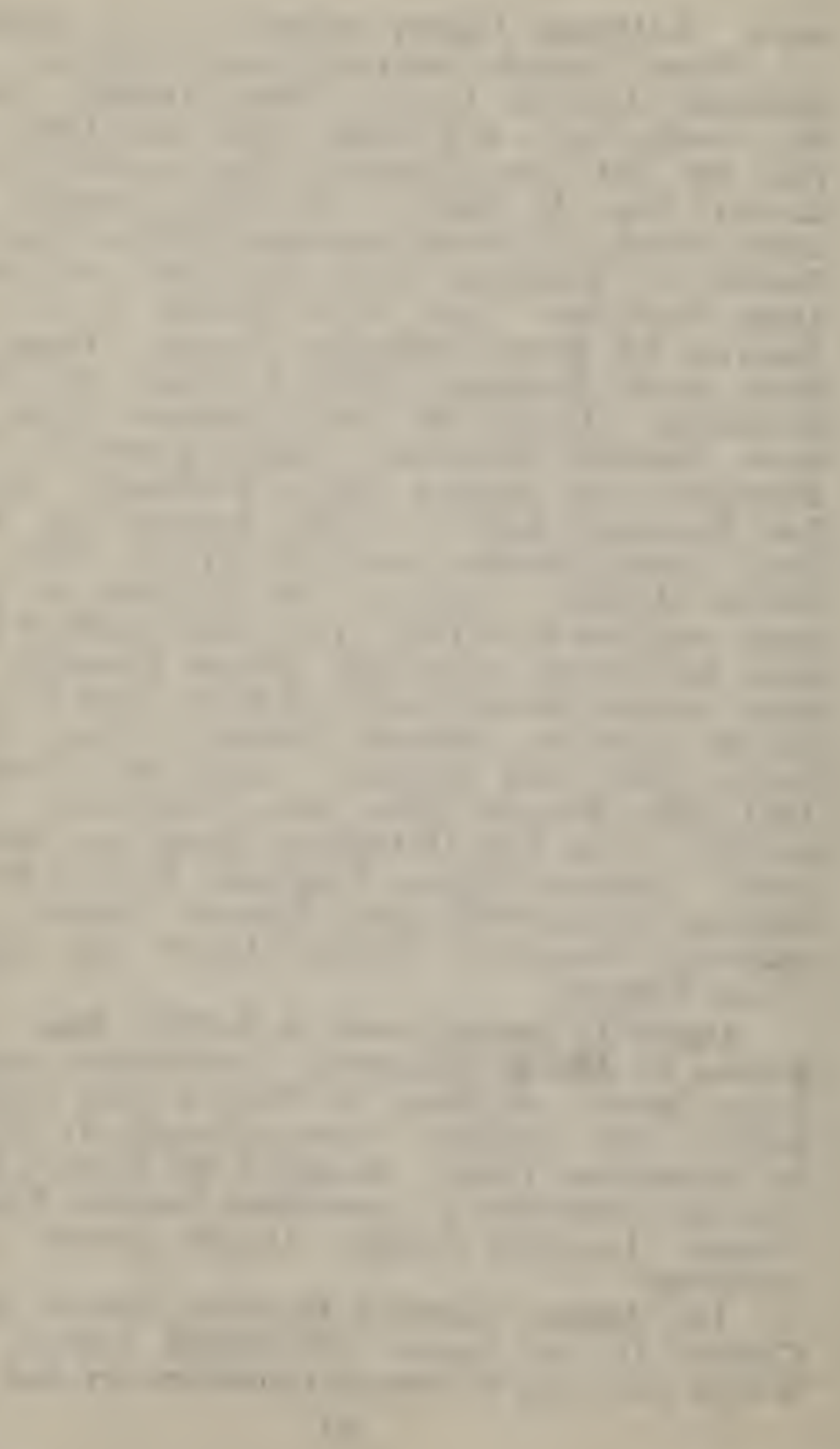


Elias Joseph, born 1885 died 1955, married Lola Mae Bryan, born 1888, died 6-30-1953. Buried in Odd Fellows Cemetery, Laurel, Del. Daughter of David and Anna M. Bryall. One son, David 13. Joseph. Married Blanche -__. One daugh.. ler, Faye Joseph. Hulda Joseph. Call Josenti. Both died when young.

Mr. Fred Joseph married 2nd. Hatlie A. Cannon, born 1878.1930 buried in Asbury Cemetery. Martin Joseph married Lillian Ryan. Six children. Harley F. Joseph, bor'n 11-25-1910, died 1-22-1950. Buried in Odd Fellows Cemetery, Lat:rel, Del. Married Mary Cullen. Two children, Froddie and Joyce Ann Joseph. Senary B. Joseph maried Guss Phillips. Died 1957. Ruth Joseph married Alvin Roland Hitchens. Five children. Roland Hitchens married Joan Wilson, daughter of Marshall and Ethel (Thompson) Wilson. Marvin. Doris. Brenda. Phyliss. Norman E. Joseph married Bertha Betts. One daughter, Elma B. Joceph. The above received from Mr's. Berthia Baker and Mrs. Mabel Foreman. Mrs. Orlando Joseph.

A. Frank Joseph, born 9-14-1861, died 4-291942, married Amelia W. Gumby 9-11-1868, died 10-30-1944.

Charles F. Joseph, born 4-6-1892, married Flossic H. Johnson, born 2-10-1893, died 4-231949. No. issue. All buried in Rehoboth Meth(xist Cemetery.

Carl T. Joseph, born 6-13-1894, married Fdith T. Fletcher, born 5-19-1895. 1-12-1959 burierl In Lowes Rehoboth Methodist Cemetery. 'T'vo sons. Carl T. Joseph, Jr. married Gill Melson. Three children, Cindy, born 10-8-1960; Yinda, born 1-0-1962; and Diane, born 3-15-63. Daughter of Ilarry E. Melson. Franklin Joseph married _Carrie Lee. Two children, John and Susan Joseph. 

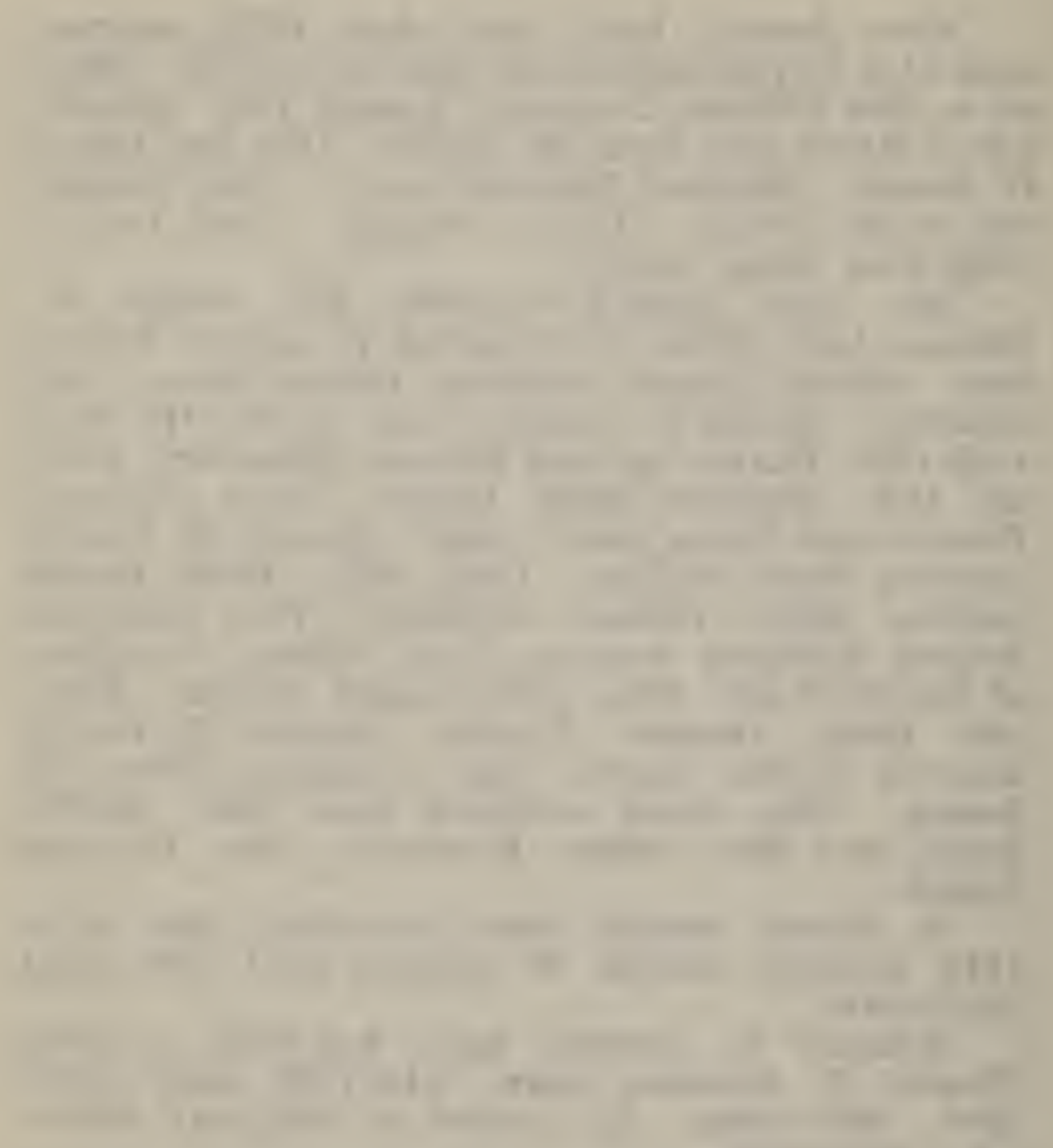

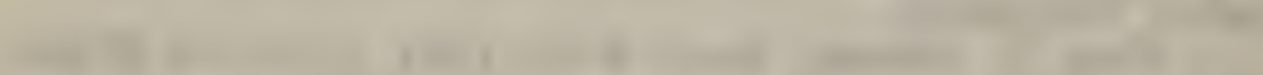

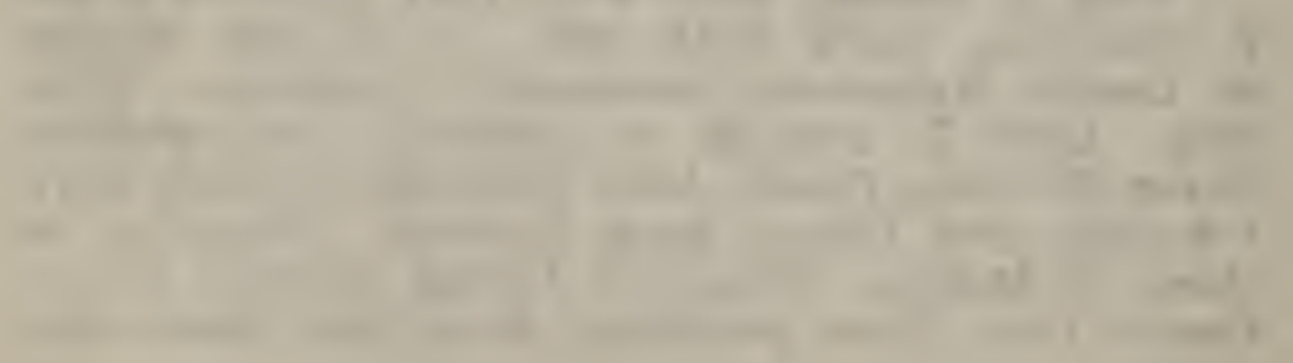


Roy Joseph married Mary Webb. A daughter, June Joseph, married Paul Lynch.

Lillie Joseph married Howard Carpenter. Five children, Sara, Elva, Ethcl, Hilda asd Agnes.

Marion Joseph married Raymond Wilson. Six children, Marion, Helen, Howard M., Ray Jr., Wayne, and Ronald Wilson deceased.

Arthur B. Joseph married Margaret Webb. One child, Wm. A. Joseph, married V. Louise - Two chiltren, Wm. Jr. and Louise.

Marrion and Arthur were twins. Chester Joseph married 1st. Anna Virginia Marvel. Two daughters. Nellie Joseph married Ralph Schmidt. Gloria V. Schmidt married Carmean Fastage. Two daughters, Fay and Nellie Fastage. Betsey Schmidt married Michael Hallenack. 'I'wo children, Michace III and Marsha Ann Hallenack. Mary $D$. Mary V. Joseph married George H. Huber. One daughter, Faye Huber. The above received from Mr. Chester Joseph of Paulsboro, New Jersey.

Lillian Minerva Joseph, 1870-1953, married Harry Jofferson, 1861-1937. One son, William A. Jefferis, 1887-1933. All buried in Lewes Mothodist Cemetery.

Jesse Edward Joseph born 1839. died 1913, married 1st Elizabeth E. West, born 1826, died 1900. Lemuel Joseph, born 1858, died 1933, masried Hannah Massey, born 1863, died 1909. All bureid in St. Georges Chapel Cemetery, Holly'mount.

Otis D. Joseph, born 1882, died 1942, married 11-20-1912 Effie C. Prettyman, born 1886, died 1943. Both buried in the same cemetery. Daughter of Wm. H. and Amanda I. (Lingo) Pretty'man. They raised Leon Reynolds.

John E. Joseph, born 1385, died 1934, married 12-22-1909 Clara W. Prettyman, 2-18-1893, daughter of Wm. H. and Amanda (Lingo) Pretty'man. Hazel Joseph married Vaughn Altemus. 


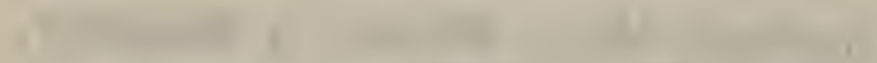

1.

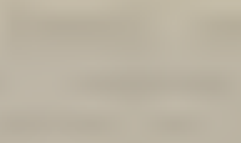

1

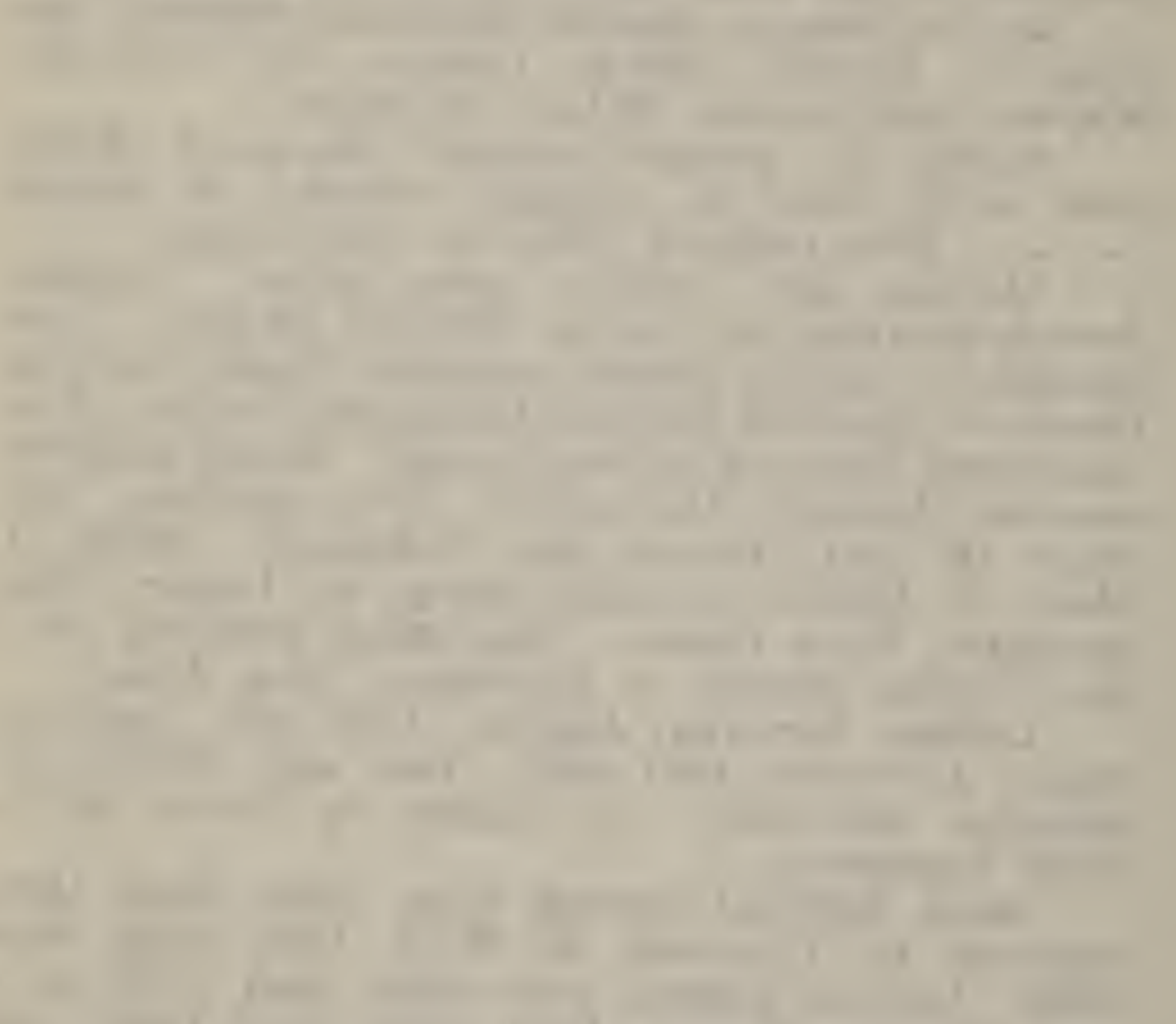

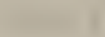

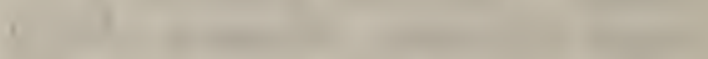

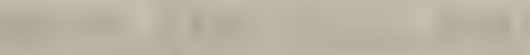

(1)

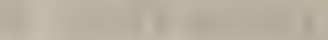

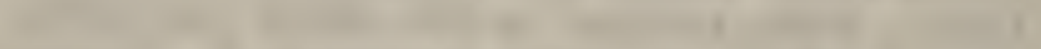

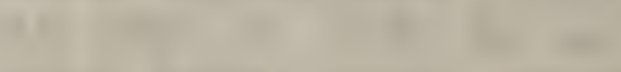

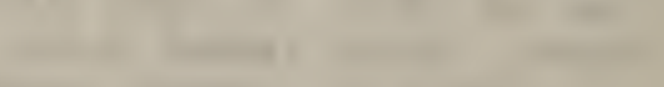

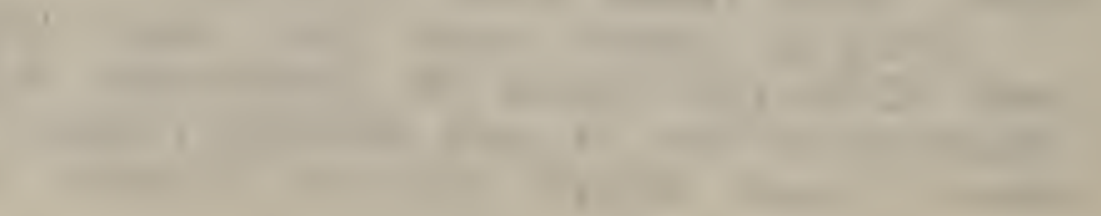

a 
Four chlldren, Leroy, Sandra, Pat, Anita Attemus. Frances Juseph married Rodney Wilgus, son o: Hundey Wilgus and Ruth Wyatt. Three children. Jujce Wilgus married __ Futcher. One daughier, lona. Janclle

Fllzabeth E. Joseph, born 1887, married Joh!1 skevenson, born 1889, died 1958. Buried at St. Cimrie's Chapel Cemetery. Son of Robert and Llaldila (Barnes) Stevenson. No issue. 'The above ufurmation received from Mrs. Elizabeth Stevenm) $n$.

Mranda Flizabeth Joseph, born 1860, died 1912. married Jehu G. Clifton, 1856-1930. Both emorled Reynolds Church Cemetery near Milton. Son of Wm. D. and Sarah Ann Cliftor.

Frank H. Clifton married Mary W. io issue. Cleveland Clifton, died 1963, buried In Reynolds Cemetery, married 1st. Ida Warringlun. born 10-23-1891, died 5-4-1955, daughter of Cerrotge and Ida Warrington. Three children, Otis J. Clifton, Edna Clifton, and Pearl Clifton. CleveLasd married 2d. Hester Hitchens. One son, Dunald Clifton. Clarence E. Clifton, born 11-22-1930, And 5-25-1955, buried in Goshen Methodist Cemeirg, Milton, Del., married Martha Calhoun, buriat In Calhoun Privte Cemetery near Milton, Del. Oixe chlld, died at the age of 10 months. Laura c7irun, deceased, buried at Smyrna, Del., married llarvey E. Wilson. Two sons, William Wilson and reurge Wilson, deceased. Virginia Clifton marrial Oscar Marvel. Five children, Alton Marvel, Chester Marvel, Mary Marvel, Clifton Marvel, anil I.fnucod Marvel. Mattie E. Clifton married Lewis smllh. Four children. The above received from Is $6 x$. Mattic Smith.

Mary Joseph, born 1864-1933, married George F. Hurdle, born 1850-1929. Both buried in St. Cinories Chapel Cemetery. Son of Jacob F. and Ilanrilh Iurdle. Jacob Hurdle was the son of 


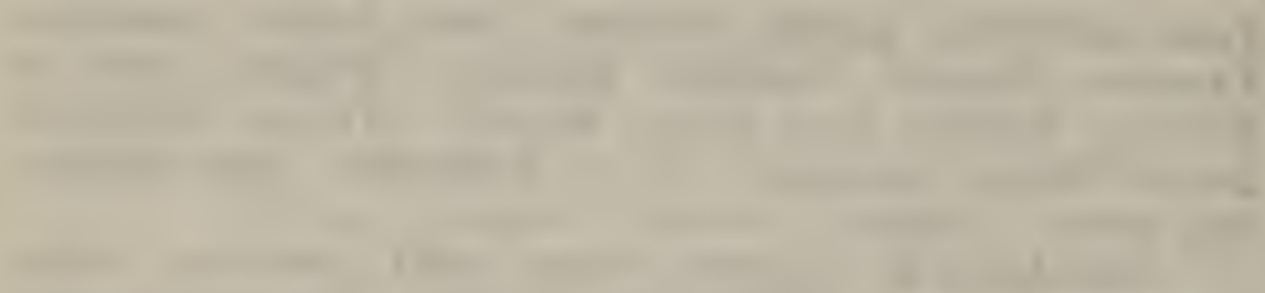

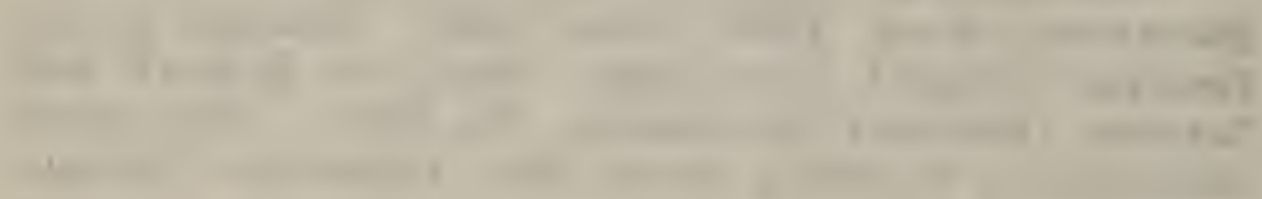

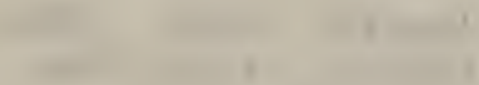

and

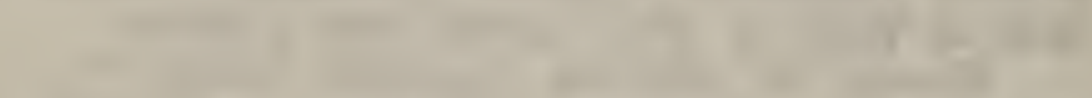

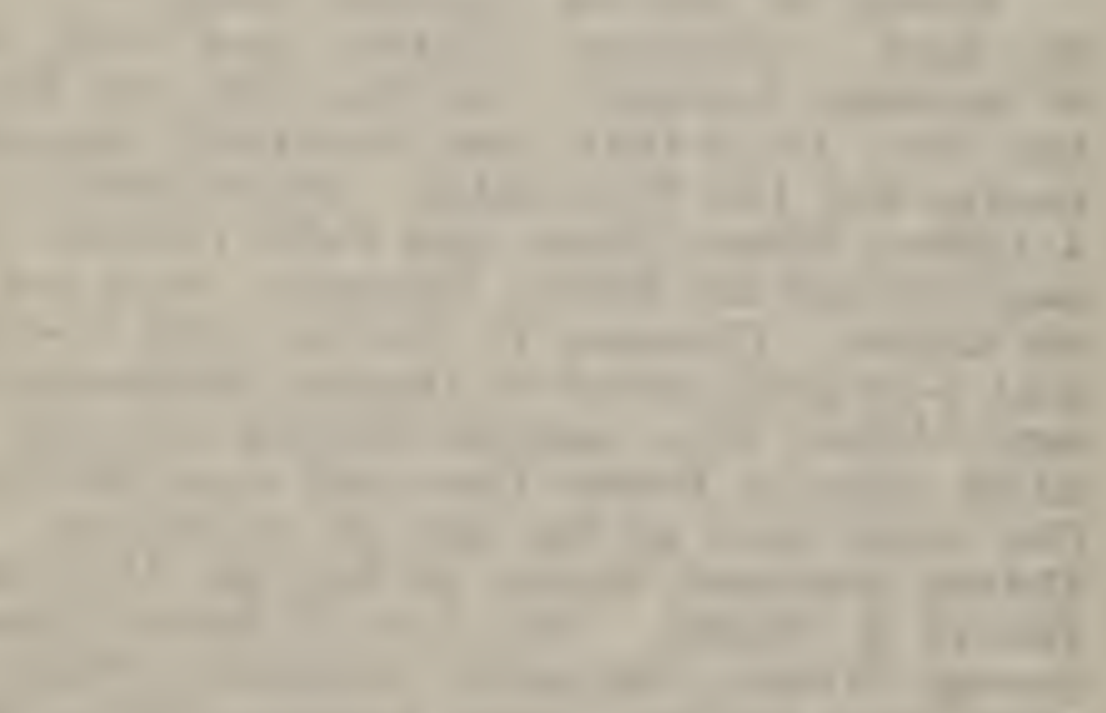

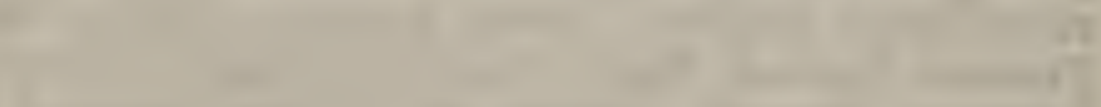
410. (4)

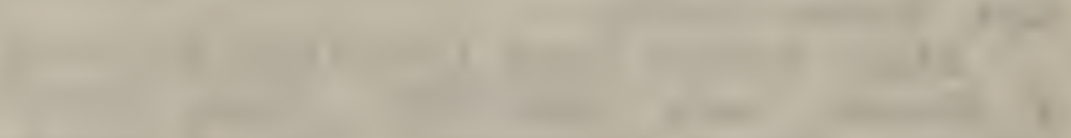
and 1 
Jacob and Valinda (Joseph) Hurdle. No issue.

Eliza Jennis Joseph, born 3-1-1866, died 1-21949, married Wm. Perry, boln 8-27-1844, died 2-22-1924. Both buried at Coolspring Presbyterian Cenctery. Son of John W. and Jane (Hopkins) Perry. William Herbert Perry, born 1-7-1882, died 12-23-1917, buried the same place, married Lilly - Basil Perry married Lina Wilsun, born 5-2-1889, died 2-10-1916. One child, Lina Perry, born 1-27-1880, died 5-6-1950. Married 2d. Georgeanna Wilson, born 5-27-1880, died 5-6-1951). Both the daughter of Jesiah and Mary (Wilkins) Wilson. One sno, Wm. J. Perry, married Maretta Conaway. 'Two children, Betty and Robert. Arthur Perry married Florence MacFirland - One child, Margaret Perry. Jessie E. Joseph had brother but we cannot find out these names at this time. We are sure he had brother but no one know's these names. Mrs. Elizabeth Joseph Steernson said that hed had brother but couldn't remember their name.

The following received from Mrs. Lyndo: Jones: We are unable to find out who Alfred Joseph's father was at this time. Alfred M. Joseph , born 1818, died 1925, married Sarah E. Joseph, born 1849, died 1920.

Gertha $W$. Joseph, born 3-12-1876, died 2-18196:, married Katie D. Lynch 4-17-1879, died 1-16-1962. Both buried Rehoboth Methodist Cemetery. Daughter of Theo. and Alice E. (Walch) L,ynch. Norma B. Joseph lives in Florida married Carrie - 'Two chilciren, Norman Joseph, Jr. and Norma Lee Joseph. Alice E. Joseph married Raymond Wilhelm. Raymond Jr. married Marge Martindale. Virginia. Dale. Robert. Alberta $V^{r}$. Joseph. Lyndon C. Jones. No issue. Son of Rev. Omar Jones. Gertrude Joseph married Norman R. Calhoun. Joy D.

Herbert B. Joseph, born 1881, died - - 


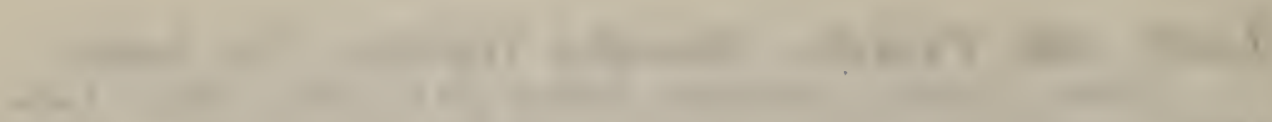

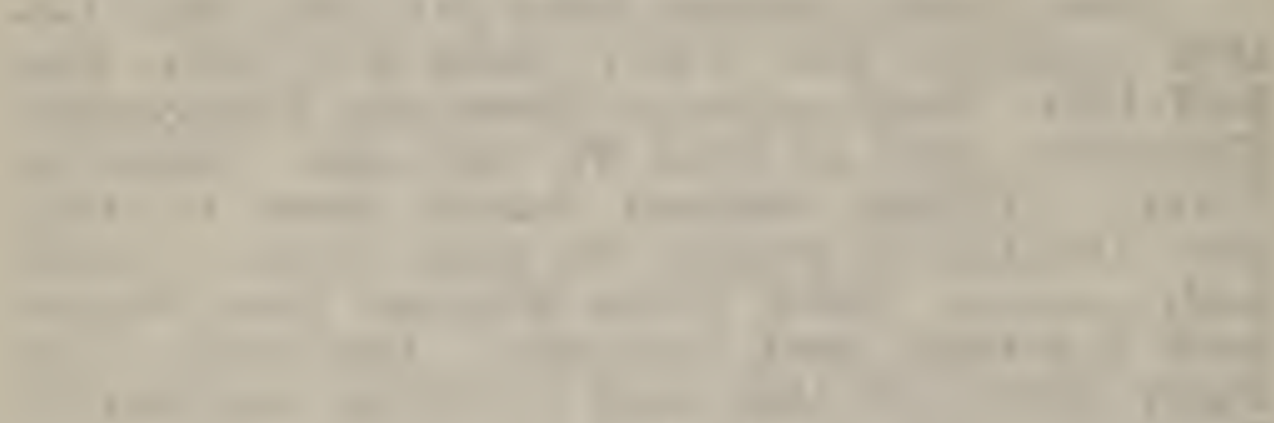

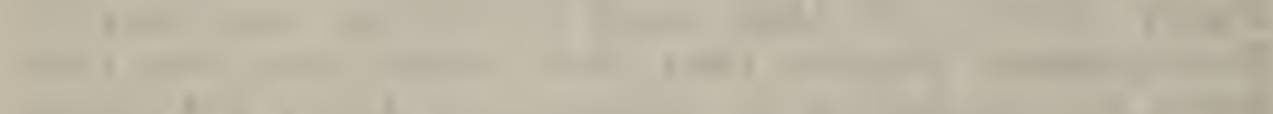

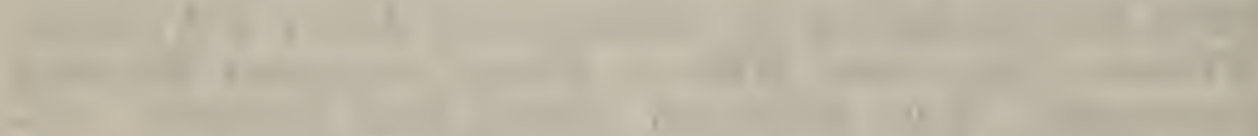

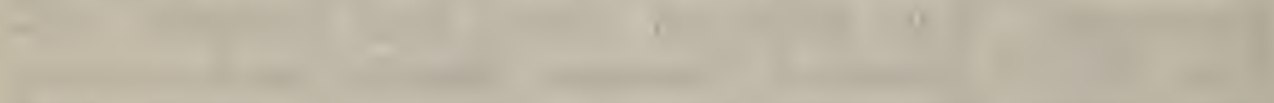

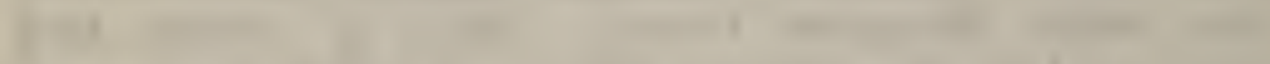
14.

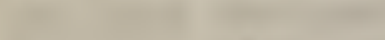 1. (1)

10

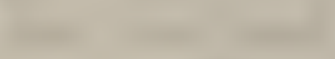

(10.90)

1

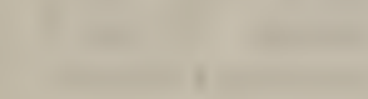

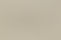

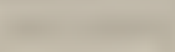

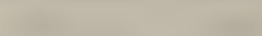

1
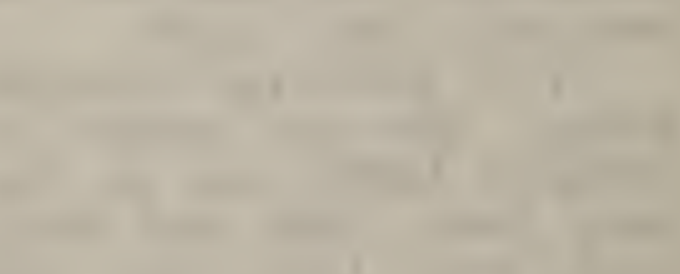

1

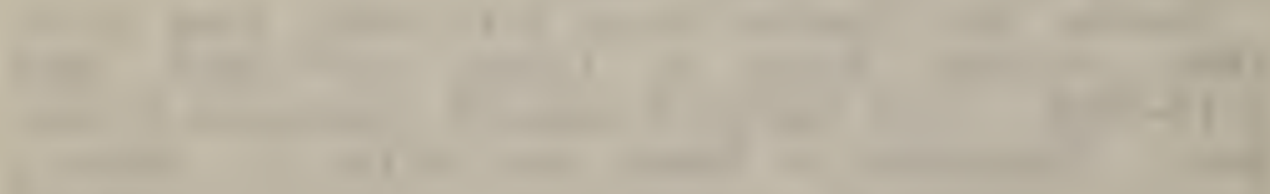
rand

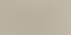

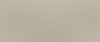

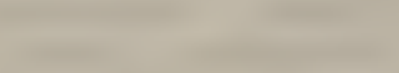

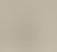

4

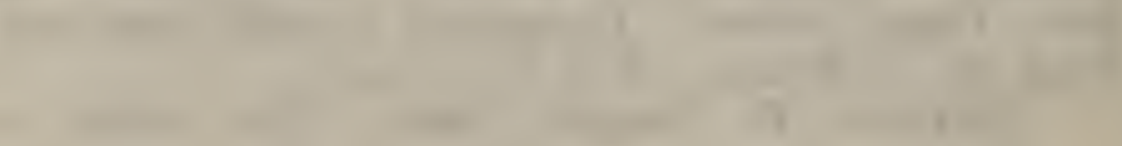


buried in Rehoboth Methodist Cemetery, married 1923 Leona E. (Berry) Russel, born 1896. No issue. Myrtle Joseph married Ralph M. Wingate, born 1890, died 1929, buried in Rehoboth Methodist Cemetery. Hazel Wingate married Larry Bowman. Sara Wingate married Berman P. Melvin. Shirley Wingate married Carl Sipp. Jay D. Wingate married _-.. Norris. Ralph $D$. Wingate, born 1921, died 1939, buried in Rehoboth Methodist Cemotery.

Sallie E. Joseph, born 2-9-1885, died 3-5-1945, buried in Rehoboth Methodist Cemetery, married Arthur J. Downnig, son of James A. and Annie Downing. One daughter, Floris Downing, never married.

Katie N. Joseph married Dr. McHenry.

Jessee E. Joseph, born 1824, died 1908, marriod 9-12-1847 Rachel A. Wingate. John W. Joseph 1861-1933 married Abbie H. - 18681940.

J. Preston Joseph, born 1895, died 12-10-196i(), all buried Lewes Presbyterian Cemetery, Lewes, Del., married Meta Green, daughter of Ralph Green. John P. Joseph married Catherine Warrington, daughter of Oscar Warrington. Elizabeth Joseph married George Lynch. Two boys. Mary I ouise Joseph maried William E. Ingram, Jr, son of Wm. S. and Florence (Scott) Ingram, Si: Willlam S. Ingram, III. Mary P. Ingram, brirn 7-li1963.

Allen B. Joseph, deceased, married 1st. Gladv's Lovelace - Married 2d. Edith Ely.

Harold B. Joseph married

Ada Joseph never married.

Erasmurs IM. Joseph, born 1852, died 1934, marricd Finma C. - born 1856, died 1929 . Ilda E. Joseph, born 6-22-1877, died 6-22-1959, married John E. Maul, born 4-20-1877, died 326-1920. Effie J. Maul, born 10-21-1904, tied 


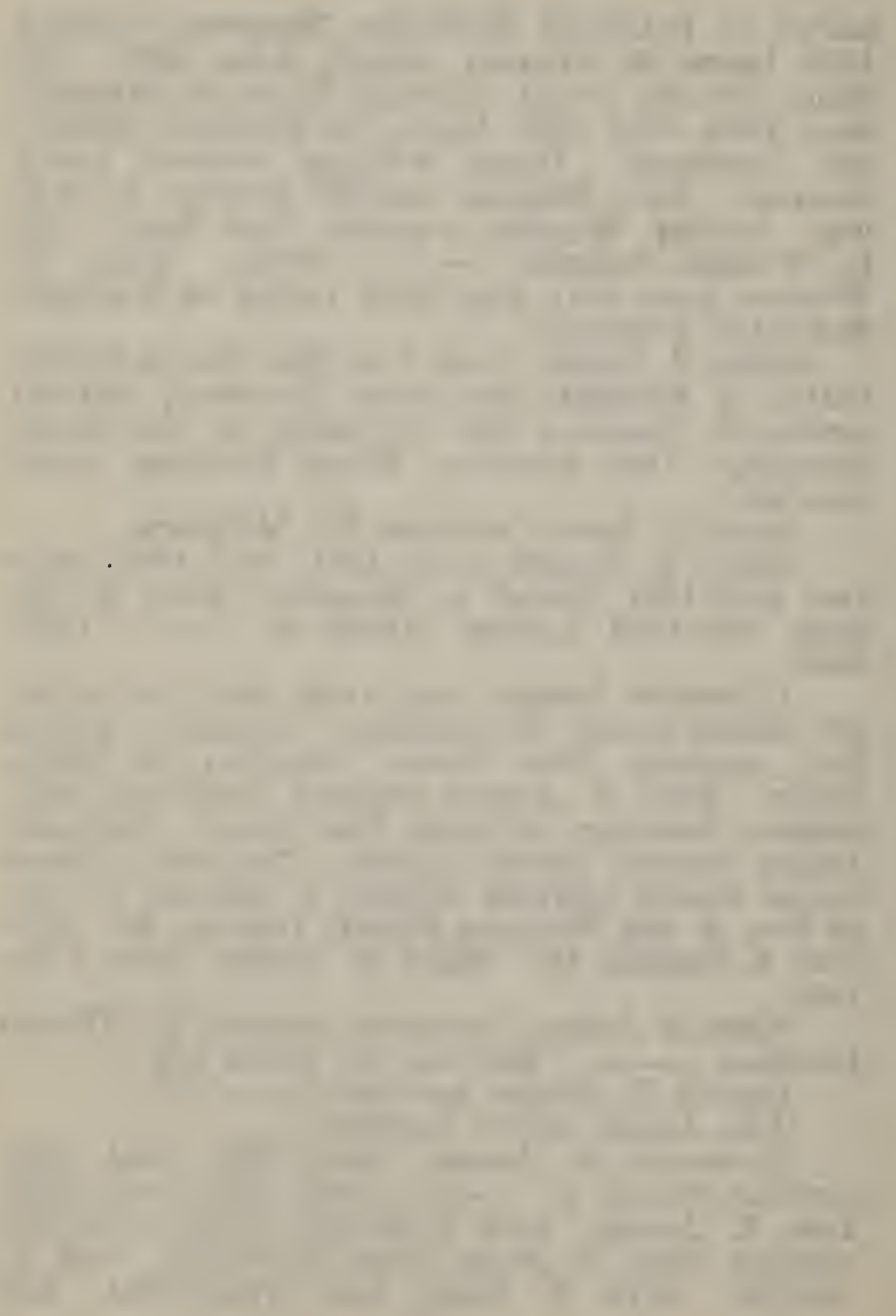


8-15-1919. All are buried in Bethel Methodist Cemetery, Lewes, Del. Martha Maul, 11-30-1933, married William Elwood Lowe. William E. Lawe, Jr. married Grace Warrington. Lucy Jean Lowe married Kenneth Band. Ella Jean Lowe. Effie C. Joseph, born 1879, died 1898, buried the same lot. Sarah Joseph married 1st. Ben Albertson, died 1952, buried St. Peters Episcopal Cemetery, Lewes, Del. Married 2d. Rev. Phillips.

The above received from Mr. Martha Lowe, Mrs. Fannie Thomas and Mrs. Preston Joseph, all of Lewes, Del.

Shepard Joseph married Mary McMullen. No issue.

Annie Joseph; born.1859, died 1935, married David H. Parks, born 1855, died 1912. Howand L Parks, born 8-16-1888, died 8-19-1889. All buried Lewes Presbyterian Cemetery, Lewes, Del. Fannie Parks married —_ Thomas, divorced.

Emma Joseph married Joseph Conwell. Capt. Arthur W. W. Conwell, 1872-1842, married Sarah J. , 1874-1960. Capt. Joseph P. Conwell, 1902-1942. Katherine C. Crutchen, 1904-1941. All buried Lewes Presbyterian Cemetery, Lewes, Del. Annie Conwell, 1875-1962, married Thomas $\mathrm{H}$. Morgan, 1864-1943. Both buried Lewes Methodist Cenetery. Elizabeth Morgan married - Howard. Arthur Morgan.

Laura Josoph, deceased, buried Lewes Methodist Cemetery, never married. Thomas W. Joseph buried l.ewes Presbyterian Cemetery, Lewes, Del., marriced Elizabeth Wingate - Had three children, all died young.

Isaac Joseph married Thomas Henry Joseph, born 3-30-1854, died 1-12-1936, marrica Lavina Caroline Joseph, born 2-8-1856, died 331-1931. Both buried Laurel Hill Cemetery. Daughter of Sallie Ann Joseph.

John E. Joseph married Mytrle

Wil- 


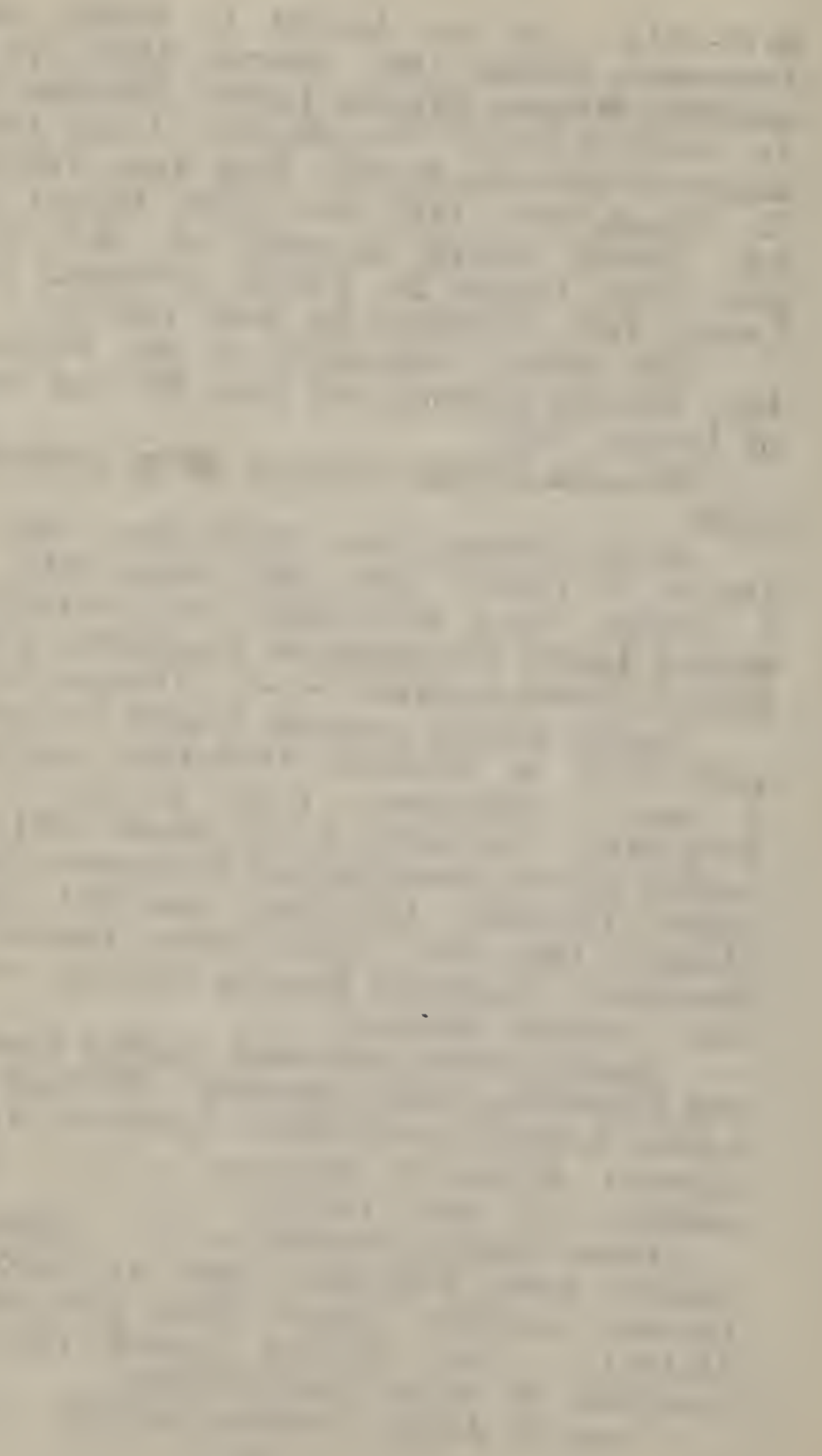


liam Joseph married Mary deceased.

Harry H. Joseph married Alice Massey. Catherine Joseph married John Phillips.

Norman B. Joseph, died 7-16-1954 - 53 years. Buried Laurel Hill Cemetery. Married Blanche C. Whaley. Five children, Ronald, Ralph, Burton, Roy, and Kathleen Joseph.

Isaac $H$. Joseph married Julia Hall. One daughter, Dafrey Joseph married Granville Lowe.

Charles L. Joseph married Susie C. Barnes, born 1903, died 10-7-1963. Buried Laurel Hill Cemetery, Laurel, Del. Three children. C. Donald Jusoph married Edith —. Dorothy Joseph married Gene Pattrson. Mary Joseph married Robert Kyttle:

Ida M. Joseph, born 8-10-1892, married Oscar W. Givens, born 12-8-1888, died 1963. Buried in Ialurel Hill Cemetery. Son of Quill and Caroline (legates) Givens. One son, Linwood Givens marrled Margaret O'Day. Two sons, James F. Givens and David Givens.

Mary Elizabeth Joseph married Franklin Hearn, drceased. Alice C. Hearn married Allison $P$. Whaley. Two ehildren. Mary Lou Whaley, born 1934, died 7-28-1963. Buried Trinity Meethodist Church Cemetery. Married Raymond M. Pusey. Norman S. Whaley. Jack Hearn, James Hearn, William Hearn, Mabel Hearn, and Gladys Hearn. Anna M. Josaph, ideceased, married John Britlingham. Both buried in Laurel Hill Cemetery. Two children. Cecie lE. Brittingham married Sillie - One child, Donald Brittingham. Myrtle Brittingham married Harvey King. One latughter.

Asher Joseph died young. Alice Joseph marrled _- Cooper. One son.

A brother to Caroline Joseph, Levi Joseph married Maggie - Both buried in Conari 


$$
-1--1+1+1
$$

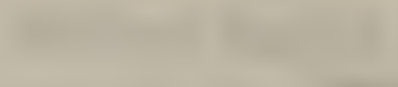

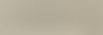

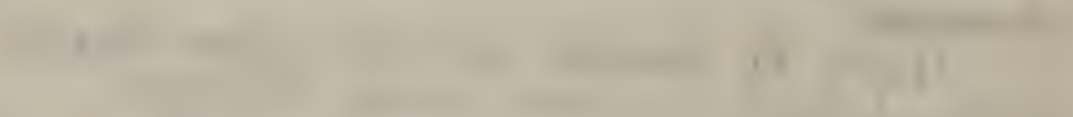

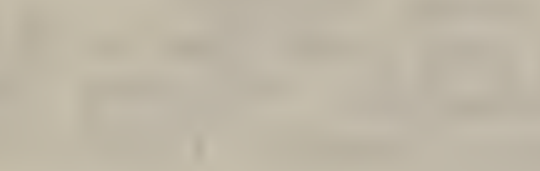

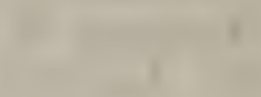

120

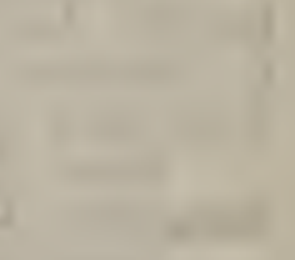

(1)

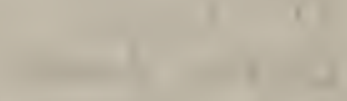

(20)

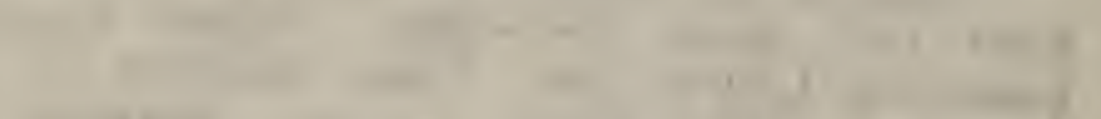

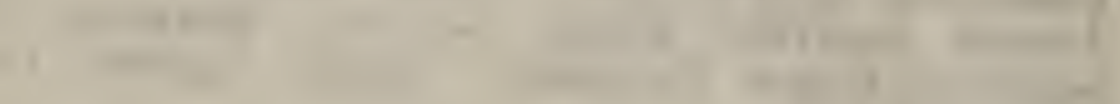

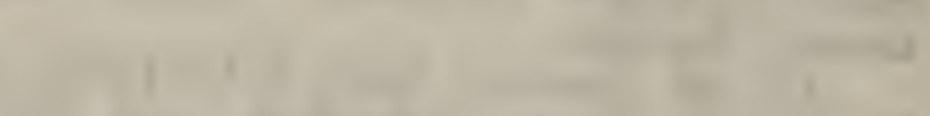

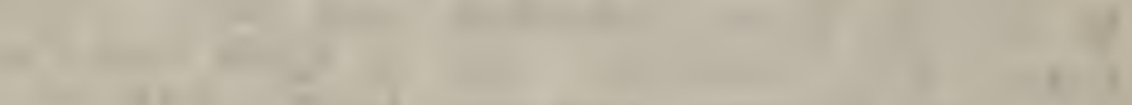
4. (1)

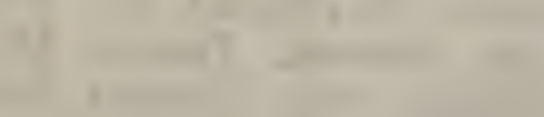

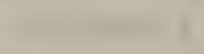

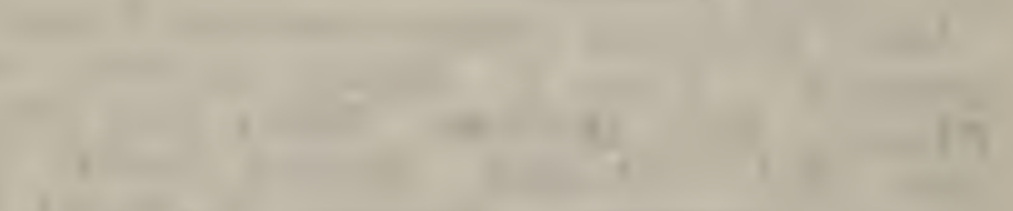

-

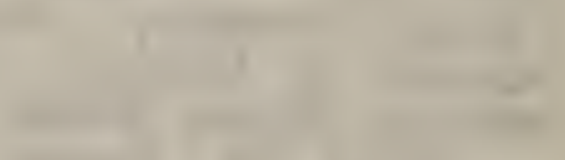

$+$

$+1$

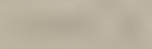

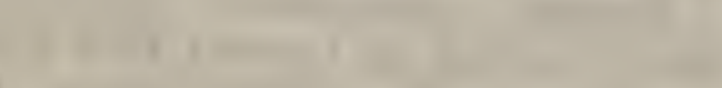
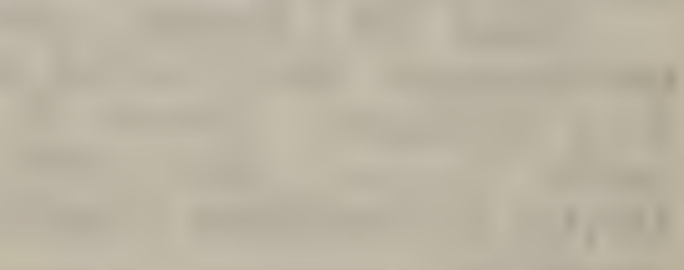

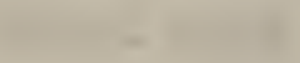

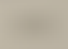

$+2$

(n)

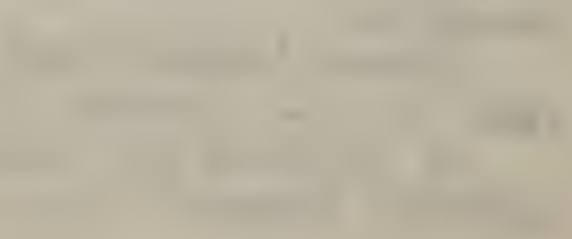

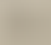


Methodist Cemetery near Seaford.

Anna Joseph, deceased, buried in Epworth Methodist Church Cemetery, married Ira Brown, his 1st. wife. James H. Joseph married Alberta M. Sallie Joseph married William Oliphant. Ida Joseph married Edward Halloway. Ella Joseph, 1875-1935, married Ernest Calloway, 1885-1953. Both buried in I aurel Hill Cemetery. Two children, John Calloway and Amy Calloway marricd Downs.

Sallie Joseph, a half sister to Thomas $H$. Joseph, married James Palmer. Two children, Linden Palmer and Ethel Palmer.

Sister to Sallie _- Millie Joseph married Erasmus Massey.

Juseph, buried in Middletown, Del., married

A half brother to Thomas Joseph, John I. Son Charles H. Joseph, 1895-1954, buried in Odd Fellows Cemetery, Laurel, married Helen - born 1896.

The above received from Mrs. Ida Givens of Laurel, Del.:

John W. Joseph, 1816-1896, married Alibice L. Hudson, 18-17, 1875. Nary A. Joseph, died 1880 - 30 ycars of age, married R. A. Hazel. Six children, Margaret, Mary, Albine, John H. Carrie, and Clarence. Carric M. Hazel married Edward C. Phillips. Elizabeth Phillips, born 12-28-1902, married Wm. H. Creadick, born 8-4-1900, son of Win. Herbert and Emma (Rollins) Creadick. One son, Edward C. Creadick married 1930 Betty O. Collins, daughter of Charles and Mary (Perry) Collins. Two children, Sandra Ray and Elizabeth Creadick.

Clarence Hazel married Annle Collison. One son, Clarence W. Hazel, born 1895, died 5-311963. Son, Clarence Hazel, Jr. married Three children. 


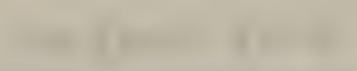

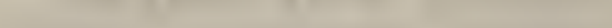

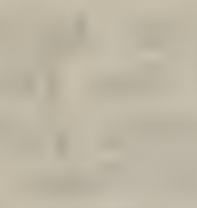
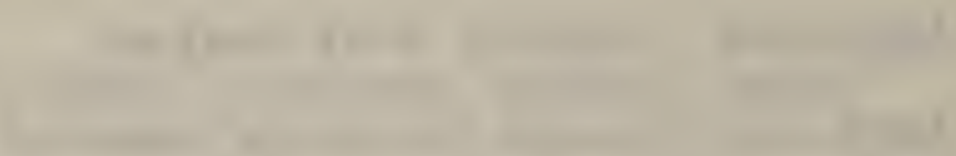

$+1$

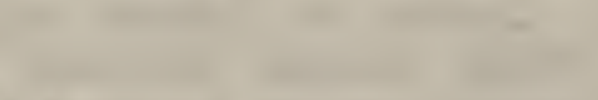

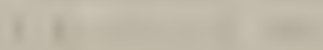


Leah Jane Joseph, born 12-24-1842, died 312-1928, never married.

William D. Joseph, 1852-1919, married Annie - 1857-1917. All are buried in Coolspring Presbyterian Cemetery. We are unable to find out any more about this family at this time.

Isaac Joseph married Rebecca - We know of one daughter, Valinda Joseph married Jacob Hurdle, born in Virginia 1780, migrated in oarly manhood to Indian River Hundred. They raised five children. Lettie Hurdle, died unmarried. Joseph C. Hurdle, born 6-8-1820, died 3-26-1892, married Eleanor Walls, born 10-10-1826, died 12-10-1889, daughter of John and Harriet (Walls) Walls.

Marcan Hurdle married Anna Joseph, daughter of David Joseph. Seven children. Lydia Ann Ilurdle , born 5-17-1853, died 1-24-1922, married David C. Lawson. Five children. John Hurdle, born 1850, died 1931, married 1st. Henrietta J. Conaway, died 6-7-1898 - 49 years. Both buried In Loives Presbyterian Cemetery. One daughter, Clara Hurdle married Georg Shutt. John marrid 2d. Theresa - born 1855, died 1925, buri(cl, Lowes Methodist Cemetery. Eliza S. Hurdle, diod 12-8-1879 - 20 years, buried in the John Walls Private Cemetery, married William Coffin. No issue. Unnamed girl child. No marker. Buried the same place.

Wm. W. Hurdle married Comfort B. Joseph. Their family is found else where in this book. Ellza Amn Hurdle married

Jacob Fisher Hurdle, 1825-1900, married Hannah - 1828-1898. Two children. George F. Hurdle, born 1850, died 1929, marricd Mary Joseph, 1864-1933, daughter of Jesse and Elizabeth (West) Joseph. Ruth M. Hurdle, born 18541891, unmarried. All buried in St. Georges Chapel Cemetery near Hollyville, Del. 


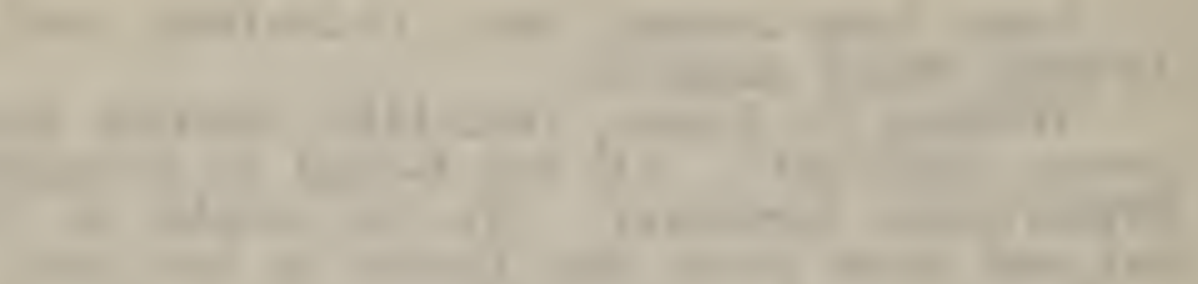

$\sqrt{2}$

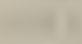

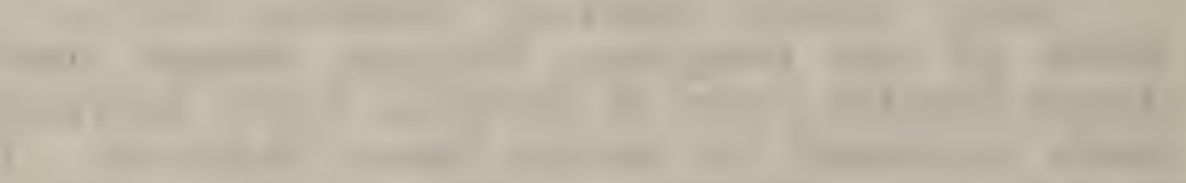

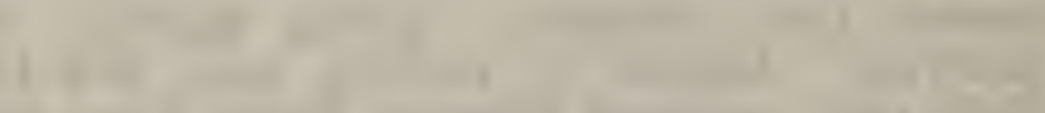

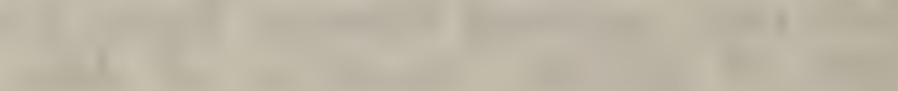

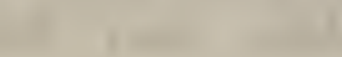

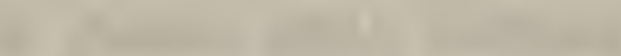
10
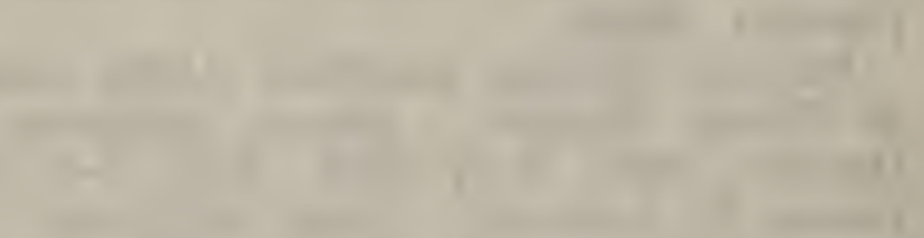

Chat +

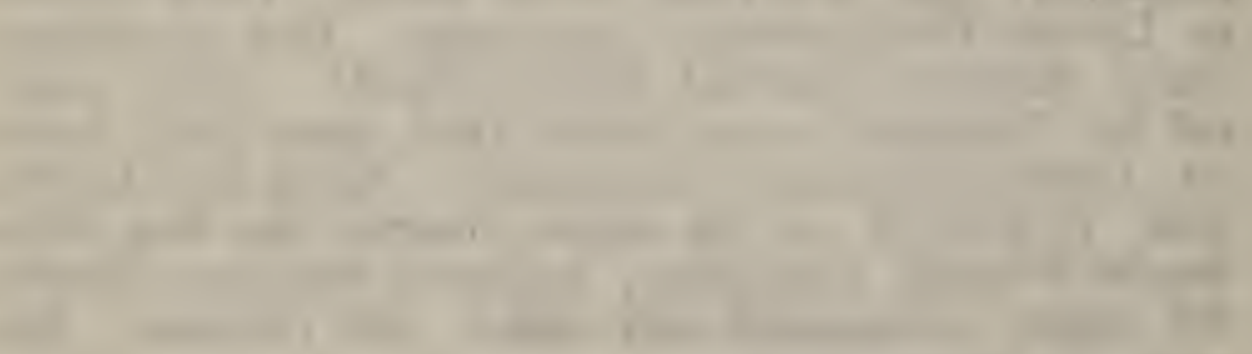

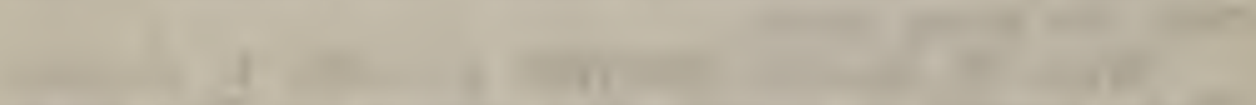

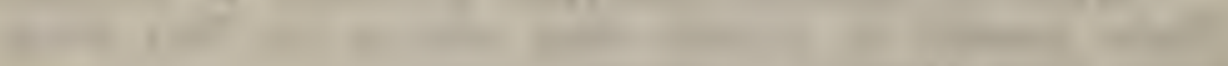

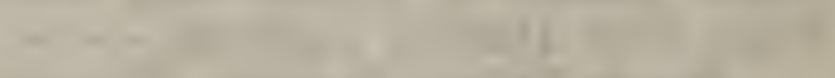

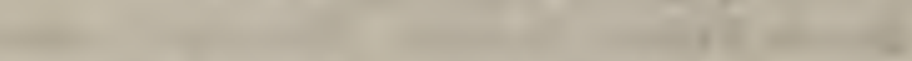

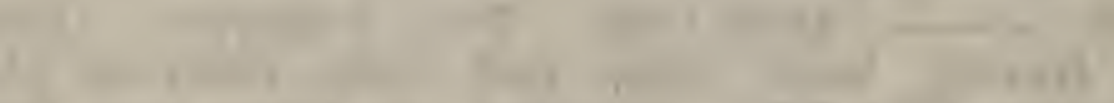

1. L. 2.

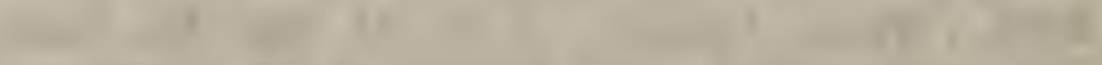

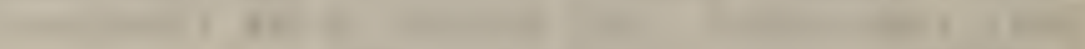


Zachariah I. Joseph, 1825-1890, married 1847 Mary D. Torbert, 1864-1883. Both buried Conley's Chapel Cemetery. 'This Josoph may be related to Jesse E. Joseph.

Ida M. Joseph, 1873-1937. Rodney Joseph, 1'910-1931. Both buried in Mechanies Cemetery, Millsboro, Del.

J. Cornelius Joseph, 1845-1914. Buried in Midway Presbyterian Cemetery near Rehoboth. Would like to know what set of Joseph he was?

Wilbert M. Joseph, died 1937 - 69 years, married 1st. Edith —_, died 1913 - 46 years. Married 2d. Susan B. Jordan, died 1963, buried at Milford, Del. They lived in Milford, Del.

There are severial Joseph that we are un. able to find any descendants. Smith Joseph married Mary Walls, born 10-27-1795, daughter of Eli and Lydia (Simpler) Walls.

Horace E. Joseph mraried Gertrude Morris, died 1949 - 68 years. Both are buried in Gracelawn Cemetery, Wilmington. Mattie Joseph, deceased, married Charles West, son of John West. Lillie Joseph married Elliott. Walston R. Joseph, died in Oklahoma. One child, died an infant. I would like to fine out more about this family. Mary.'E. Joseph, 1st. wife of U. G. Jeseph. Married 2d. Catherine V. Joseph, 18671936. Both buried, Lewes Methodist Cemetery.

J. B. Joseph, livde in Milford, married Sarah Walls, daughter of Burton and Elizabeth Walls.

Thomas H. Joseph, born 1786, died 6-8-1864, married Hannah _- born 1791, died 4-14-1865. This is on her tombstone. She devoted her life to the service of her master 54 years. Hannah Jos!h 1825-7-21-187\%. Annie E. Joseph died in infancy. All buried on a fam cemetery near Halleyville. Elizabeth Joseph married __- Lingo. Nohemiah Joseph. Wm. W. Joseph.

Comfort B. Joseph, 1819-1867, married 1-23- 


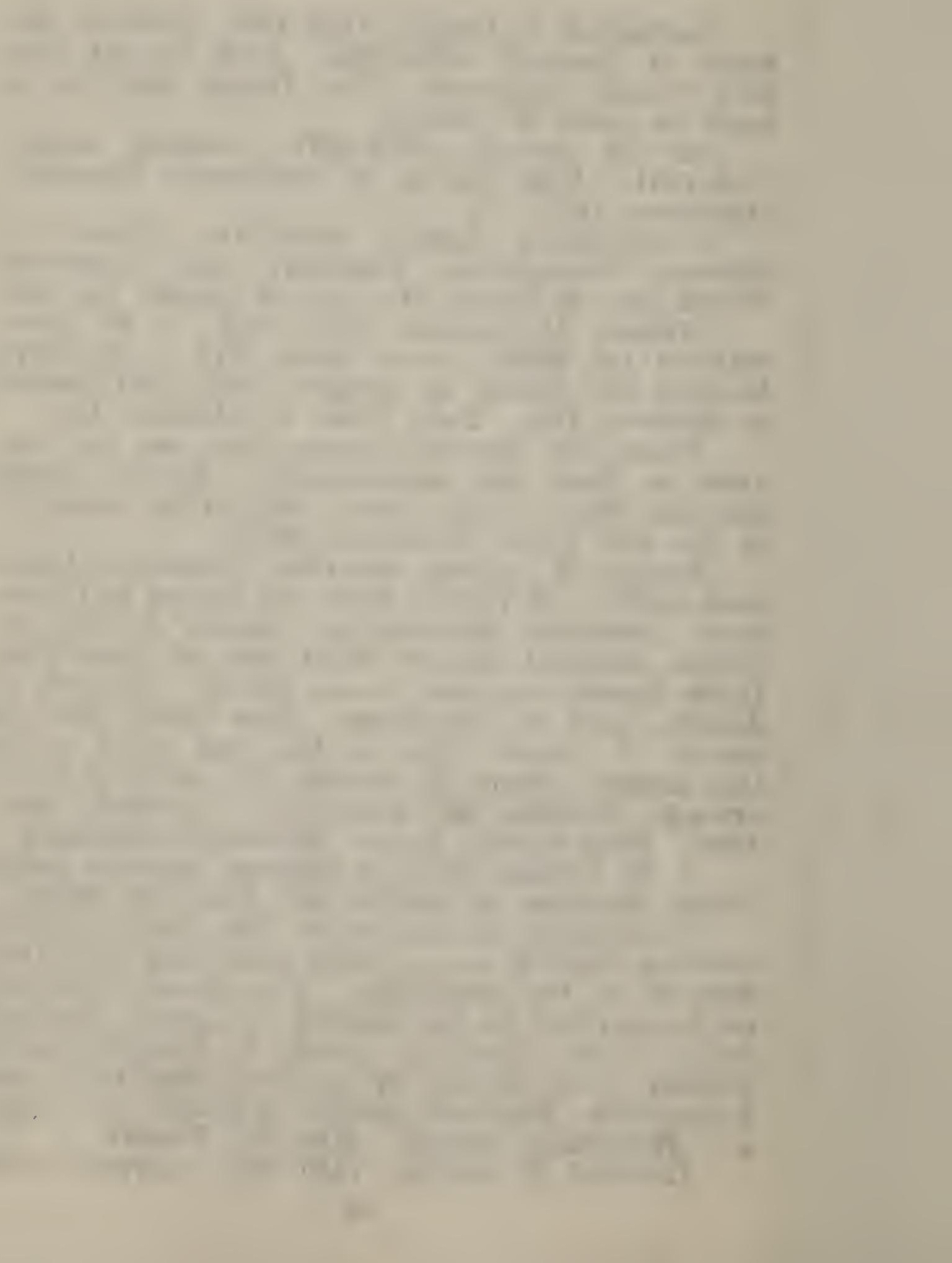


1843 William W. Hurdle, 1822-1885, son of Jacob and Valinda (Joseph) Hurdle. Joseph Hurdle, 1854-1859, (Mr. Wm. WW. Hurdle married ¿d. Hester Goslee). George W. Hurdle, 1852-1871. Vilanda Hurdle, born 7-27-1871, died 9-22-1871. Annie E. Hurdle ,born 6-20-1851, died 1-29-1870, married Silas W. Warrington. Isabell Hurdle, 1348-1868, married David P. Warrington, born 186 7 1881. All buried at St. Georges Chapel Cemetery. Amanda P. Hurdle, born 5-9-1857, died 1220-1892, buried in Coolspring Presbyterian Cemetery, married John S. Veasey, born 1854, died 1926. He is buried in Harbeson Cemetery, son of - Georgia Veasey, 1877-1957, married 1st. James C. Martin, born 9-20-1874, died 3-121910. Boht buried in Coolspring Presbyterian Cemetery. She married 2d. Doyle. Three ohildren by 1 st. husband, Lloyd Martin, Madeline Martin, and James C. Martin. Virginia Veasey, born 1883, died 3-26-1963, married Clarence Robert Kidwell. . Both buried in Wilmington, Del. No issue. Bessie H. Veasey, born 7-23-1891, married William F. Wilson, 1891-1956. Buried in Union Cemetery, Georgetown. Son of Josiah S. and Mary A. (Wilkins) Wilson. Lillian Wilson maried John Alton Warrington, born 3-22-1928. Five childrer, Bonnie Lee W. married - Wyatt, Nancy Lee W. married - Reed, one child, Geo. Alton Reed; Allan Warrington, Charles D. Warrington, and Gale Louise Warrington. Marion C. Wilson married Harold Scott, killed in the service. Three childmen. Edwin D. Wilson married Pauline Workman. One daughter, Diane. Elwood Wilson married Helen' Spencer, deceased. 'Two children. Daughter of Clarence and Clara (Short) Spencer. Carole Wilson married Marshall Stepp. One child, Kenneth Stepp. Richard Wilson. Russell Wilson married Beverly Justice, daughter of Elmer R. and Alberta (Mitchell) Justice. Three children, 


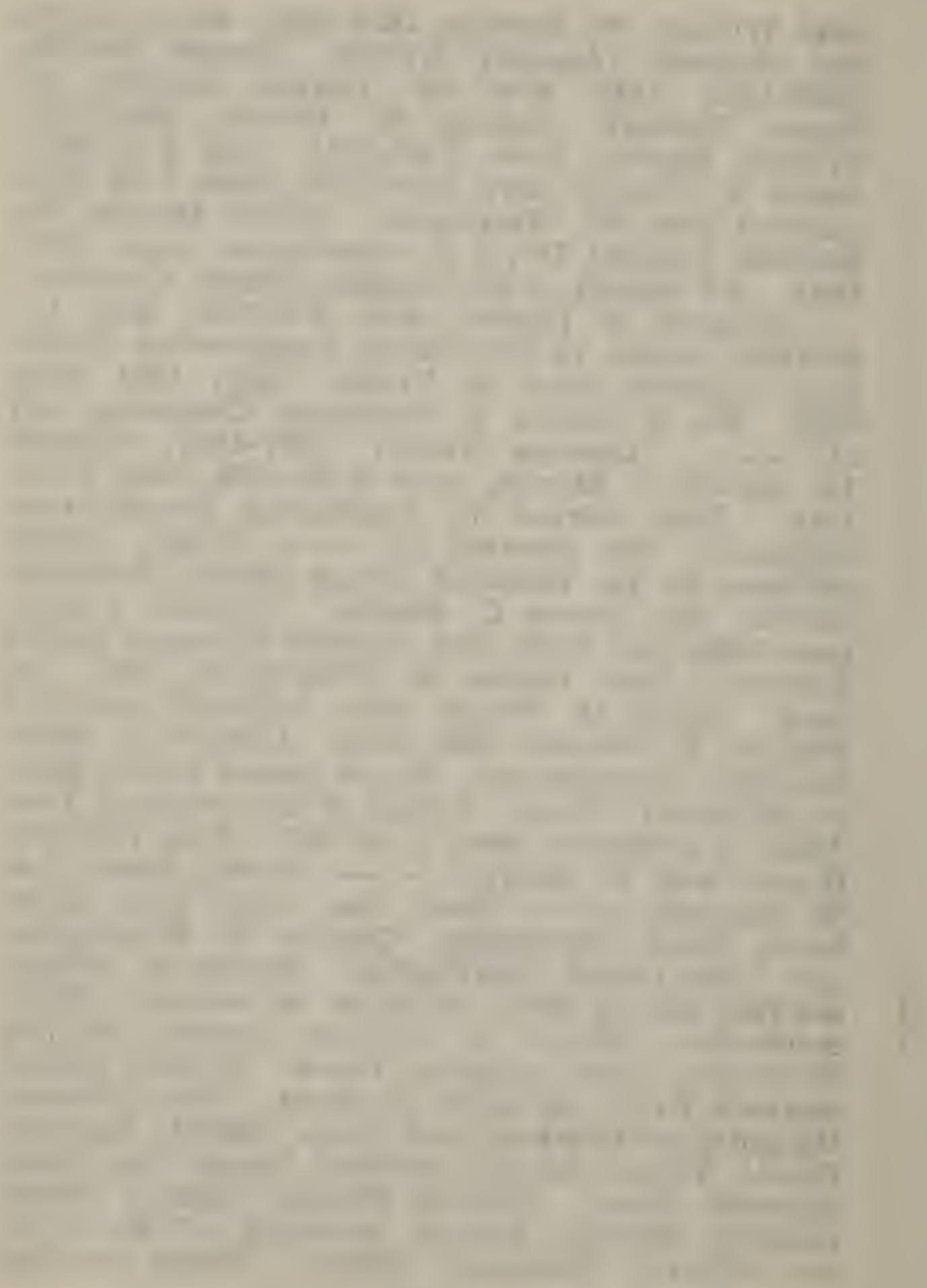


Michael, Debbie and Donna. Delma Wilson married Wm. Q. Mitchell. Five children, Robert, Judy, Wayne, Wrn. Jr. and James.

William Thomas Hurdle, born 1850, died 1919, buried in Lewes Methodist Cemetery, married Arrbelle Prettyman, died 1875 - 23 years, buried in St. Georges Chapel Cemetery. One daughter, Arrie Hurdle married Watter Virden. Wm. Inarried 2d. Sallie H. Perry. No issue. Daughter of John M. and Jane (Hopkins) Perry. Wm. married $3 d$. Cornelia, born 1878, died 1943, burled in Lewes Methodist Cemetery. Two children, Wn. Thomas Hurcle, Jr. married Alvine M. One son, Wm. Q. Hurdle, III. Cornelia Hurdle, unmarried.

This information received from Mrs. Bessie Wilson; Mrs. Lillian Warrington. 
10
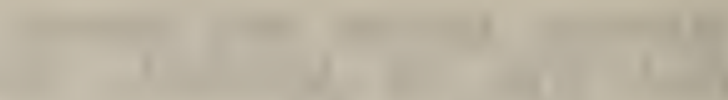

100

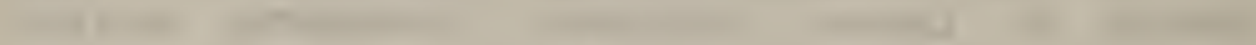

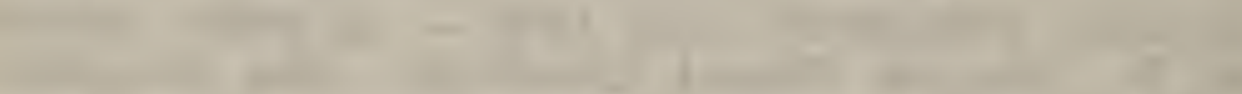

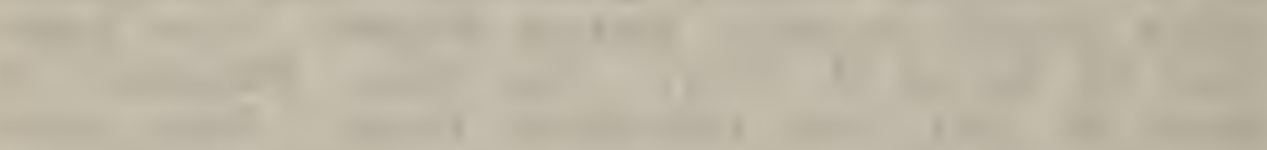

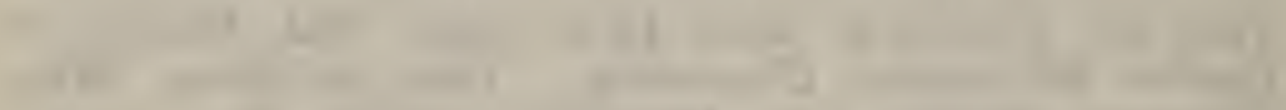

$\sqrt{20}$

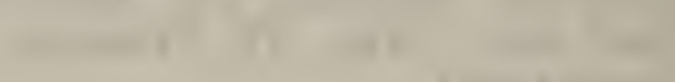

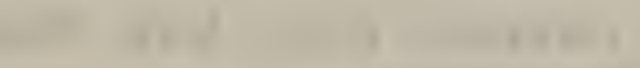

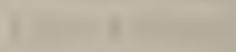

(1)

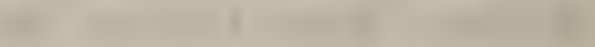





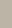



